\title{
Partikelmodellierung der Strukturbildung akustischer Kavitationsblasen in Wechselwirkung mit dem Schalldruckfeld
}

\author{
Dissertation \\ zur Erlangung des Doktorgrades \\ der Mathematisch-Naturwissenschaftlichen Fakultäten \\ der Georg-August-Universität zu Göttingen \\ vorgelegt von \\ Philipp Koch \\ aus Göttingen
}

Göttingen 2006 
D7

Referent:

Prof. Dr. Werner Lauterborn

Korreferent:

PD Dr. Martin Rein

Tag der mündlichen Prüfung:

29. August 2006 


\section{Inhaltsverzeichnis}

1 Einleitung und Gliederung $\quad 1$

2 Radiale Blasendynamik 9

2.1 Blasenpulsationen . . . . . . . . . . . . . . . . . . . . . . 11

2.1.1 Minnaert'sche Erweiterung . . . . . . . . . . . . . . . 13

2.1.2 Erweitertes Rayleigh-Modell . . . . . . . . . . . . . . . . 14

2.1.3 Keller-Miksis-Modell . . . . . . . . . . . . . . . . . . . 15

2.1.4 Typische Blasenschwingungen . . . . . . . . . . . . . . 17

2.2 Blake-Schwelle . . . . . . . . . . . . . . . . . . . . 21

2.3 Polytropenexponent. . . . . . . . . . . . . . . . . 25

2.4 Oberflächenmoden . . . . . . . . . . . . . . . . . . . 32

2.5 Diffusion . . . . . . . . . . . . . . . . . 37

3 Struktur des Schallfeldes $\quad 47$

3.1 Analytische Beschreibung . . . . . . . . . . . . . . . 50

3.2 Eigenmoden . . . . . . . . . . . . . . . . . . . . . . 52

3.3 Blasen-Schallfeld-Wechselwirkung . . . . . . . . . . . . 58

3.3.1 Wijngaarden-Modell . . . . . . . . . . . . 58

3.3.2 Numerische Lösungen im Eindimensionalen . . . . . . . . . 62

3.3.3 Linearisierung . . . . . . . . . . . . . . . . . . . . . . . . . . 65

3.3.4 Lösungen im Dreidimensionalen . . . . . . . . . . . . . . 69

3.3.5 Einzelblase in der Küvette . . . . . . . . . . . . . . . . 74

3.4 Laufendes Wellenfeld . . . . . . . . . . . . . . . . . . 78

4 Translationsdynamik $\quad 81$

4.1 Virtuelle Masse . . . . . . . . . . . . . . . . . . . . . . . 83

4.2 Primäre Bjerkneskraft . . . . . . . . . . . . . . . . 84

4.3 Sekundäre Bjerkneskraft . . . . . . . . . . . . . . . . . . . . . . . . 97

4.4 Reibungskraft . . . . . . . . . . . . . . . . . . . . . 107

4.5 Partikelmodell . . . . . . . . . . . . . . . . . 111 
4.5.1 Programmablauf ... . . . . . . . . . . . 113

5 Anwendungen der Partikelmodellierung 116

5.1 Lasererzeugte Einzelblasen . . . . . . . . . . . . . . . . . . . . . . 118

5.1.1 Partikelmodellierung . . . . . . . . . . . . . . . . 123

5.2 Zweiblasen-Wechselwirkung . . . . . . . . . . . . . . . . . . 128

5.2.1 Partikelmodellierung . . . . . . . . . . . . . . . . . . 129

5.2.2 Abspaltung . . . . . . . . . . . . . . . . . . . 131

5.3 Dreidimensionale Filamentfigur . . . . . . . . . . . . . . 133

5.3.1 Partikelmodellierung . . . . . . . . . . . . . . . 137

5.4 Erfassung der Blasenradien . . . . . . . . . . . . . . . . . 142

5.5 Doppelschichtige Blasenfigur . . . . . . . . . . . . . . . . 147

5.5.1 Numerische Simulation . . . . . . . . . . . . . . . . . 148

5.6 Kreuzförmige Streamerfigur . . . . . . . . . . . . . . . . . . . . . 154

5.7 Blasen-Schallfeld-Rückkopplung . . . . . . . . . . . . . . . . . 157

5.7.1 Numerische Analyse . . . . . . . . . . . . . . . . . . . 158

5.7.2 Partikelmodellierung . . . . . . . . . . . . . 163

6 Zusammenfassung und Ausblick 171

$\begin{array}{ll}\text { A Symbolverzeichnis } & \mathbf{1 7 5}\end{array}$

A.1 Tabellenwerte . . . . . . . . . . . . . . . . 177

A.2 Naturkonstanten . . . . . . . . . . . . . . . . 177

A.3 Kennzahlen . . . . . . . . . . . . . . . . . . . . . . 178

$\begin{array}{lr}\text { B Gerätespezifikationen } & 179\end{array}$

B.1 Küvettensysteme . . . . . . . . . . . . . . . . . . . 179

B.2 fs-Laser System . . . . . . . . . . . . . . . . . . . . 181

B.3 Beleuchtungen . . . . . . . . . . . . . . . . . . 182

B.4 Aufnahmesysteme . . . . . . . . . . . . . . . . . 182

B.4.1 Kameras . . . . . . . . . . . . . . . . . . . . . 182

B.4.2 Objektive . . . . . . . . . . . . . . . . 183

B.4.3 Hydrophone . . . . . . . . . . . . . . . . . . . . . 184

$\begin{array}{ll}\text { Literaturverzeichnis } & 185\end{array}$

Daumenkino auf Seite 169 


\section{Kapitel 1}

\section{Einleitung und Gliederung}

Die vorliegende Arbeit untersucht die Modellierung von akustisch induzierten Kavitationsblasenstrukturen. Hierzu werden eine Partikelmodellierung für die Simulation der Blasenbewegungen und die Methode der Finiten Elemente zur Lösung der das Schallfeld beschreibenden Wellengleichung eingesetzt. Die enge Wechselwirkung von Blasendichte und Schallfeld wird dabei berücksichtigt.

\section{Kavitation}

Begrifflich leitet sich Kavitation von dem lateinischen cavitas ab und bezeichnet das Phänomen der Bildung von Hohlräumen in Flüssigkeiten. Dabei entstehen Blasen, die sich mit Dampf und mit in der Flüssigkeit enthaltenen anderen Gasen füllen. Eine grundlegende physikalische Einführung gibt etwa Trevena [1]. Bei schallinduzierter Kavitation haben diese Gasblasen typischerweise eine Größe von wenigen Mikrometern. Zu radialen Pulsationen angeregt, zeigen sie dann ein Schwingungsverhalten, bei dem sie ein Vielfaches ihres Ruheradius erreichen können. Abhängig von Parametern wie dem Ruheradius und dem treibenden Druck, kann im Verlauf der Blasendynamik ein relativ starker Kollaps auftreten. Dieser Kollaps ist oft der Grund für beobachtete Kavitationsbegleiterscheinungen.

Entsprechend der möglichen Enstehungsmechanismen von Kavitation wurde von LAUTERBorn $[2,3]$ die in Abbildung 1.1 gezeigte Gliederung des Kavitationsphänomens angegeben.

Historisch wurde zuerst die hydrodynamische Kavitation beobachtet. Anfang des zwanzigsten Jahrhunderts wurden von SilberRAD [4] Schädigungen an Schiffspropellern den auftretenden Kavitationsblasen zugeschrieben (sogenannte Kavitationserosion). Ebenso können Schädigungen an Düsen, Turbinen oder

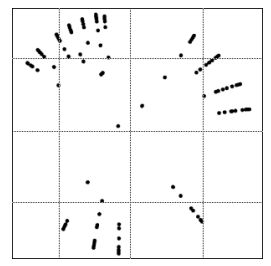




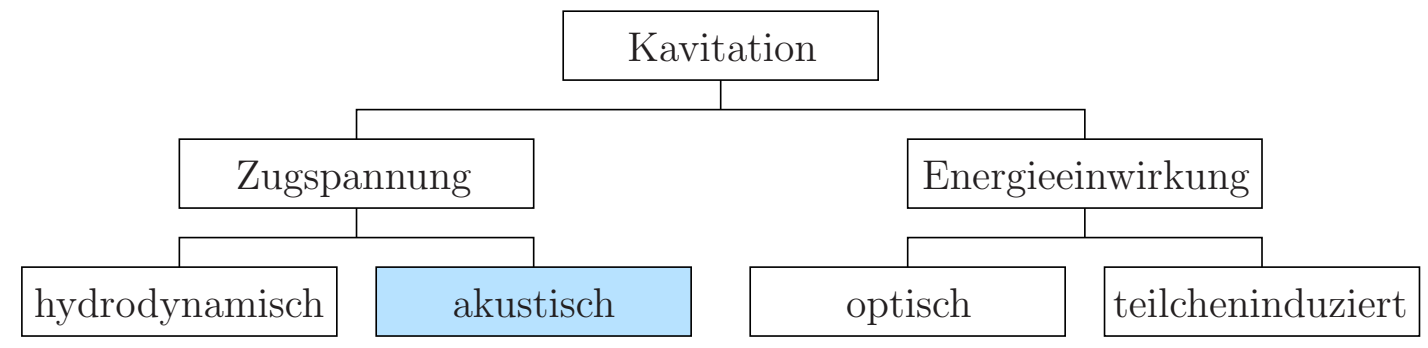

Abbildung 1.1: Gliederung der Kavitations-Erscheinungsformen. In dieser Arbeit wird die akustische Kavitation als Folge von in Flüssigkeiten auftretender Zugspannungen durch ein Ultraschallfeld betrachtet; Einteilung nach LAUTERBORN $[2,3]$.

hinter Ventilen auftreten, wo durch die Strömungsgeometrie negative Drücke auftreten können und die Flüssigkeit aufreißt (kavitiert), diese Erscheinungen werden zum Beispiel von KnAPP et al. [5] beschrieben.

Über einen Ultraschallwandler kann Schall in die Flüssigkeit eingekoppelt werden. Bei hinreichender Intensität kommt es auch hier in den Unterdruckphasen zu einem Aufreißen der Flüssigkeit, diese akustische Kavitation dokumentieren etwa Young [6], Leighton [7] oder BREnNEn [8].

Optische Kavitation lässt sich durch einen stark fokussierten Laserstrahl in der Flüssigkeit induzieren, wie es beispielsweise von VOGEL und LAUTERBorN [9] eingesetzt wurde. Im optischen Durchbruch kommt es zur Plasmabildung, mit dessen Ausdehnung sich eine Kavitationsblase ausbilden kann.

Teilcheninduzierte Kavitation wird durch den Energieeintrag eines eingeschossenen Elementarteilchens ausgelöst, zum Beispiel aus der kosmischen Höhenstrahlung oder einer Neutronenquelle. Dabei kommt es durch den Energieeintrag bei der Kollision mit Flüssigkeitsmolekülen zum Aufreißen von Hohlräumen, RozENBERG [10] beschreibt beispielsweise diesen Effekt.

\section{Akustische Kavitation}

Das Aufreißen der Flüssigkeit durch starke Schallfelder wird als akustische Kavitation bezeichnet (Young [6], Leighton [7]). Sie wird etwa in der Sonochemie zur Katalyse chemischer Reaktionen eingesetzt (MAson et al. [11]). Auch die Reinigung mittels Ultraschall (BERGMANN [12]) basiert auf akustischer Kavitation. Weitere Anwendungen betreffen die Medizin. In Wechselwirkung kollabierender Blasen mit biologischen Zellen kann beispielsweise eine zeitweise Poration der

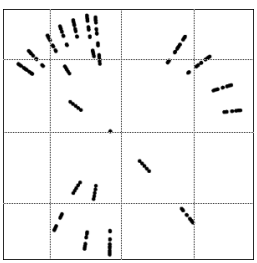


Zellen erreicht werden, ohne diese dauerhaft zu schädigen, so dass ein Wirkstoff eindringen kann (Kodama et al. [13], Schlicher et al. [14]). Verwandte Phänomene betreffen Kontrastmittelbläschen, die gezielt durch Blutbahnen geleitet werden, um mittels an den Blasen reflektierter Schallwellen den Blutstrom verfolgen zu können oder um in die Blasen eingeschlossene Medikamente gezielt lokal freisetzen zu können (Postema et al. [15]).

In den meisten Anwendungen treten die akustischen Kavitationsblasen nicht homogen im Raum verteilt auf, sondern zeichnen sich durch Inhomogenitäten und Strukturen aus. Vielblasensysteme bilden relativ komplexe Strukturen aus, diese Dynamik untersuchte zum Beispiel PARLitz et al. [16].

Überschreitet die Intensität des eingekoppelten Ultraschalls die Kavitationsschwelle, so kommt es zum Aufreißen der Flüssigkeit. Bevorzugt entstehen Blasen an den sogenannten Kavitationskeimen (Rozenberg [10]). Dies können stabilisierte Mikroblasen oder kleine Lufteinschlüsse sein, die etwa Staubpartikeln anhaften. Auch Objektflächen und Behältniswandungen fungieren mitunter als Blasenquellen.

Abbildung 1.2 verdeutlicht die Zusammenhänge einiger wichtiger Faktoren, die Einfluss auf die Strukturbildung akustischer Kavitationsblasen nehmen.

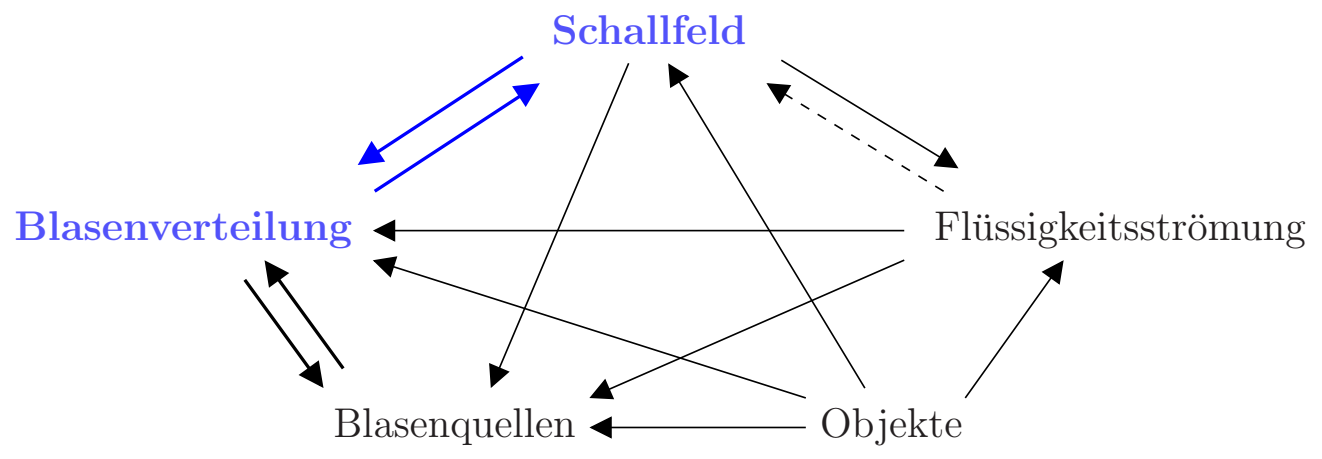

Abbildung 1.2: Einflussfaktoren auf die Ausbildung der akustischen Kavitationsblasenstrukturen. In dieser Arbeit wird vorrangig die gegenseitige Einflussnahme zwischen der Blasenverteilung und dem Schallfeld betrachtet.

In experimentellen Versuchen zeigen die Blasenverteilungen die Ausbildung verschiedener Strukturen, die zum Teil aus dem Translationsverhalten der Blasen folgen. In der vorliegenden Arbeit wird die Strukturbildung durch den Einsatz der Partikelmodellierung untersucht.

Die Wechselwirkung zwischen dem eingekoppelten Schallfeld und der Blasenverteilung wird in der folgenden Arbeit vorrangig behandelt. Über die sogenannten

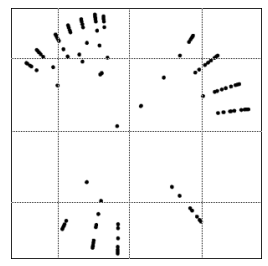


Bjerkneskräfte werden die Blasen in dem Schallfeld beschleunigt. Umgekehrt koppelt die Verteilung der Blasen durch Veränderung der Eigenschaften des Mediums auf die Schallfeldverteilung zurück. Zur Beschreibung dieses Effekts wird eine modifizierte Wellengleichung eingesetzt.

Die von dem Schallfeld aktivierten Blasenquellen nehmen durch ihre Position und die Entstehungsrate neuer Blasen Einfluss auf die Blasenverteilung. Umgekehrt können sich Blasenkeime von Blasen lösen und die Blasenverteilung so Einfluss auf die Verteilung der Quellen nehmen.

Etwaige Objekte, die sich in dem Flüssigkeitsbad befinden, können Einfluss auf das gesamte System nehmen. Das Schallfeld kann an ihnen gestreut werden, so dass sich keine klare Modenstruktur ausbilden kann. Die Blasenverteilung und die Flüssigkeitsströmung können umgelenkt werden. Außerdem können eingetauchten Objekten Blasenkeime anhaften.

Durch das Schallfeld werden auch Flüssigkeitsströmungen verursacht. Diese können Blasen und Blasenkeime transportieren. Da ihre Geschwindigkeit bei den in der vorliegenden Arbeit betrachteten Problemen in der Regel deutlich unterhalb der Schallgeschwindigkeit anzusetzen ist, ist kein signifikanter Einfluss auf das Schallfeld zu erwarten. Im Folgenden wird die Auswirkung der Strömung auf die Blasenverteilung, die Blasenquellen und das Schallfeld vernachlässigt. Ebenso wird die Wechselwirkung mit Objekten nicht betrachtet und Blasenkeime als ortsfest angesehen.

\section{Ultraschallreinigung}

Ein Anwendungsgebiet der akustischen Kavitation, das diese Arbeit entscheidend motiviert hat, ist die Reinigung mittels Ultraschall. Diese erfolgt durch den Effekt der Kavitationserosion, der etwa von Tomita und Shima [17] oder Philipp und Lauterborn [18] untersucht wurde. Eine kollabierende Blase sendet eine Stoßwelle aus. Außerdem formt sich beim Kollaps in der Nähe einer Grenzfläche ein Wasserstrahl. Diesen beiden Phänomenen wird eine abtragende Wirkung auf das Oberflächenmaterial zugeschrieben. In Abbildung 1.3 ist beispielhaft die sich über einige Sekunden fortsetzende Abtragung von einer mit Lasertoner beschichteten Folie im Kavitationsfeld zu verfolgen.

Die Inhomogenität der Blasenverteilung und damit der Reinigung ist ein häufiges Problem in der Anwendung.

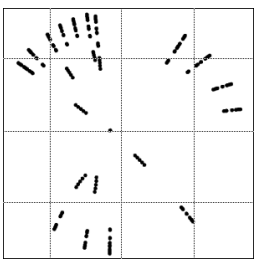




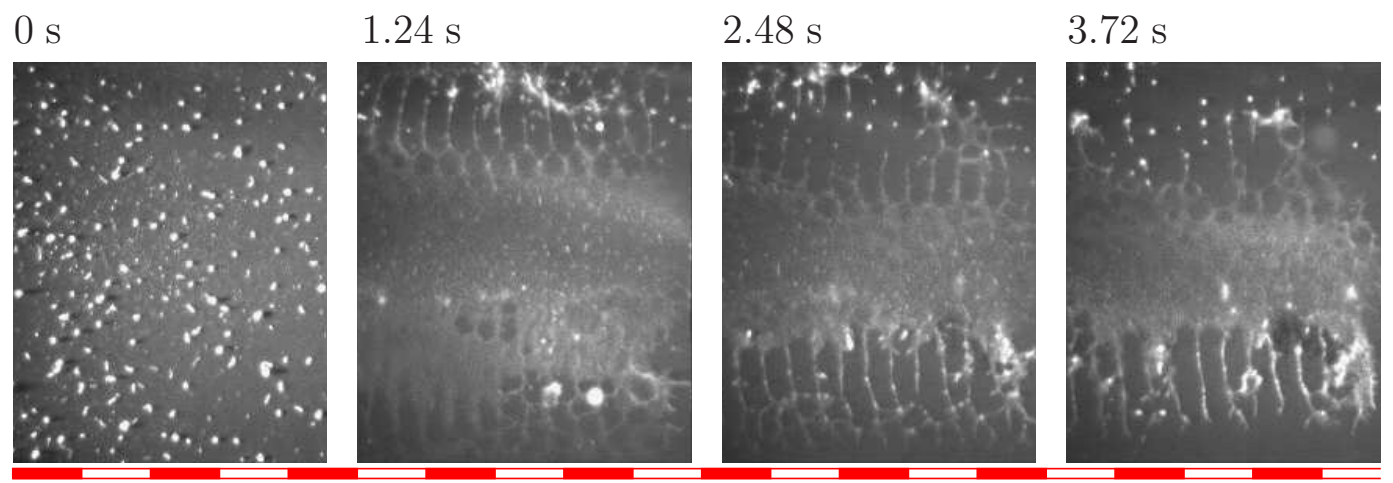

$5 \mathrm{~mm}$

Abbildung 1.3: Exemplarische Abtragung von auf einer Folie aufgebrachtem Lasertoner. Mit Einschalten des Ultraschalls $(t=0 \mathrm{~s})$ kommt es zur großflächigen Bildung zahlreicher Kavitationsblasen (helle Punkte). Die Strukturbildung der Blasen ist in den folgenden Zeitschritten zu erkennen. Durch ihre Anordnung führen sie zu einem regelmäßigen Muster in dem die Probe gereinigt wird. Aufnahme von Krefting [19]; Auflösung $115 \mu \mathrm{m} /$ Pixel, $\nu=40 \mathrm{kHz}$.

Die Betrachtung der Abtragung zeigt, wie in Abbildung $1.3 \mathrm{zu}$ sehen, relativ strukturierte Einwirkungen der Kavitationsblasen. Diese Arbeit soll das grundlegende Verständnis für das Zusammenspiel von Blasenstrukturen und Schallfeld verbessern und durch die Modellbildung typische auftretende Blasenverteilungen beschreiben. Längerfristiges Ziel ist unter anderem eine gezielte Beeinflussung und Optimierung der Kavitationswirkungen. Ein erstes Beispiel hierfür ist etwa die von SoвоттA et al. [20] beschriebene „akustische Bürste“, wobei Blasenstrukturen durch entsprechende Ansteuerung der Ultraschallwandler verschoben werden können.

\section{Gliederung}

Die radiale Blasendynamik einer einzelnen Blase wird zunächst in Kapitel 2 betrachtet. Dabei wird für den Parameterbereich von Ruheradius $1 \ldots 20 \mu \mathrm{m}$ und Schalldruckamplitude $1 \ldots 300 \mathrm{kPa}$ in einem Frequenzbereich um $20 \mathrm{kHz}$ die Blasendynamik in Bezug auf die folgenden Punkte diskutiert:

2.1 Aus der Rayleigh'schen Betrachtung der sphärischen Blasenpulsation leitet sich mit Erweiterungen von Minnaert, Plesset und Poritsky das KellerMiksis-Modell ab. Dieses Modell beschreibt die nichtlineare Schwingungen

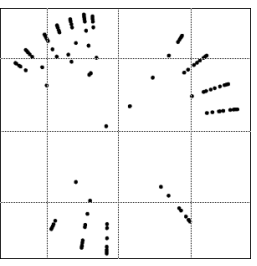


Kap. 1 Einleitung und Gliederung

rein sphärischer Blasen in einem schwach kompressiblen Fluid.

2.2 Die Blake-Schwelle charakterisiert das Einsetzen einer deutlich nichtlinearen Pulsation.

2.3 Die Wahl des Polytropenexponenten für das Gas in der Blase verlagert die Pulsation zwischen isothermem und adiabatischem Prozess.

2.4 Mit dem Anschwingen von Oberflächenmoden können stark schwingende Blasen ihre Oberflächenstabilität und damit ihre Kugelform verlieren.

2.5 Die gerichtete Diffusion kann je nach Parameterbereich ein Anwachsen oder Auflösen der Blase bedingen.

Das Kapitel 3 geht auf die Struktur des Schallfeldes ein. Zur Beschreibung der raum-zeitlichen Schallfeldausbildung ist die Wellengleichung zu lösen, die in zeitharmonischer Betrachtung in die Helmholtz-Gleichung übergeht:

3.1 In der analytischen Beschreibung des Schallfeldes lässt sich explizit eine Lösung der Helmholtz-Gleichung für die vorhandene Geometrie einer quaderförmigen Küvette mit Randbedingungen angeben.

3.2 Die Eigenmoden einer quaderförmigen Küvette werden untersucht und die Dämpfung des Systems an die experimentelle Resonanzkurve angepasst.

3.3 Zur Beschreibung der Blasen-Schallfeld-Wechselwirkung wird die Wijngaarden-Wellengleichung aufgestellt, die die Schallausbreitung im Zweiphasengemisch beschreibt. Sie wird mittels der Methode der Finiten Elemente (FEM) gelöst, wodurch auch komplizierte Geometrien untersucht werden können. Betrachtet wird ebenfalls die Resonanzverschiebung eines Küvette-Blase-Systems schon mit dem Auftreten einer einzelnen Blase im Zentrum („Blasenfalle“).

3.4 Bei der Betrachtung einer Ultraschall-Sonotrode wird ein laufendes Wellenfeld angenommen.

Kapitel 4 widmet sich der Translationsdynamik von Blasen. Die Trennung von der schnelleren in Kapitel 2 behandelten Pulsationsdynamik ist aufgrund der langsameren Zeitskala der Blasentranslation möglich. Die Bewegungsgleichung wird aus vier Kräften aufgebaut:

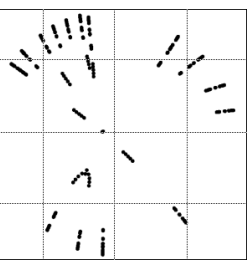


4.1 Die virtuelle Masse berücksichtigt die durch die Strömung der Flüssigkeit um die Blase hervorgerufene Trägheit.

4.2 Durch die primäre Bjerkneskraft wird die Kraftwirkung des Schallfeldes auf die Blase beschrieben.

4.3 Die sekundäre Bjerkneskraft steht für die Wechselwirkung benachbarter Blasen.

4.4 Die Viskosität bewirkt eine Reibungskraft.

4.5 Schließlich wird das implementierte Partikelmodell aufgestellt.

Kapitel 5 stellt Anwendungen der Partikelmodellierung vor. Zur Simulation von Blasenstrukturen werden die in den vorangegangenen Kapiteln vorgestellten Modellsysteme verwendet:

5.1 An lasererzeugten Einzelblasen wird die Modellierung der experimentell erfassten Blasentrajektorien demonstriert.

5.2 Anhand von zwei aufeinander folgenden laserinduzierten Blasen wird die Zweiblasen-Wechselwirkung untersucht.

5.3 Die Ausbildung einer komplexeren dreidimensionalen Filamentfigur wird quantitativ von der Modellierung wiedergegeben.

5.4 Die Erfassung der Blasenradien bedarf der Rückrechnung experimentell aufgenommener Blasen auf ihren Ruheradius.

5.5 Eine spezielle doppelschichtige Blasenfigur wird in ihren Eigenschaften nachgebildet.

5.6 Die Entwicklung einer durch feste Vorgabe der Blasenentstehungspunkte initialisierten kreuzförmigen Streamerfigur wird modelliert.

5.7 Bei der Blasen-Schallfeld-Rückkopplung wird die sich aus der momentanen Blasenverteilung ergebende Resonanzverschiebung eines Resonator-Systems betrachtet und damit eine niederfrequente Fluktuation der Struktur nachgebildet.

Kapitel 6 gibt eine Zusammenfassung der Arbeit sowie einen Ausblick über Fragestellungen, die im Rahmen dieser Arbeit nicht behandelt werden konnten.

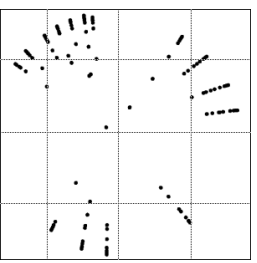


Die Arbeit schließt mit Anhang A und B. Anhang A beinhaltet ein Symbolverzeichnis, welches die verwendeten Zeichen und gegebenenfalls den Wertebereich der physikalischen Größe angibt. Anhang B führt die Gerätespezifikationen der in Experimenten zum Einsatz gekommenen Apparaturen auf.

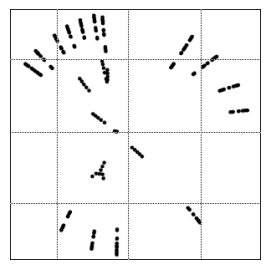




\section{Kapitel 2}

\section{Radiale Blasendynamik}

Um später die Dynamik vieler Blasen in Strukturen modellieren zu können, muss zunächst das Verhalten einer einzelnen Blase bekannt sein. Hierzu wird die radiale Oszillation einer als rein sphärisch angenommenen Blase betrachtet.

Im Abschnitt 2.1 wird zunächst das von Rayleigh aufgestellte Modell einer nicht getriebenen, sphärischen, leeren Blase eingeführt. Die Erweiterung von Minnaert auf eine gasgefüllte Blase ist in Abschnitt 2.1.1 gezeigt. Weitere Ergänzungen der ursprünglichen Rayleigh'schen Blasenmodellierung von Plesset und Poritsky beziehen die Oberflächenspannung und die Viskosität ein (Abschnitt 2.1.2). Schließlich wird in Abschnitt 2.1.3 auf das Modell nach Keller und Miksis eingegangen, welches die radiale Blasendynamik in einem schwach kompressiblen Fluid beschreibt.

Die Keller-Miksis-Gleichung liefert die nichtlineare, radiale Pulsationsdynamik getriebener, sphärischer Gasblasen in einem viskosen, schwach kompressiblen Fluid:

$$
R=R\left(p_{\mathrm{A}}, R_{\mathrm{n}}, t\right)
$$

$R$ Blasenradius

$p_{\mathrm{A}} \quad$ Wechseldruckamplitude am Ort der Blase

$R_{\mathrm{n}} \quad$ Ruheradius der Blase

$t$ Zeit

Numerische Lösungen dieses in der weiteren Arbeit eingesetzten Keller-MiksisModells zeigt Abschnitt 2.1.4 für den in der Ultraschallkavitation interessanten Parameterbereich.

Die in Abschnitt 2.2 erläuterte Blakeschwelle definiert den parametrischen Übergang von einer schwachen Pulsation zu einer stark nichtlinearen radialen Blasendynamik. 
Betrachtet wird außerdem die Bedeutung des Polytropenexponenten nach Devin (Abschnitt 2.3).

Abschnitt 2.4 zeigt die Stabilität einer Blase bei anschwingenden Oberflächenmoden.

Durch die Wirkung der gerichteten Diffusion, wie sie in Abschnitt 2.5 im Wesentlichen der Beschreibung von Eller und Flynn folgend behandelt wird, kommt es zu einem zeitlich veränderlichen Ruheradius der Blase:

$$
R_{\mathrm{n}}=R_{\mathrm{n}}(t), \quad R_{0}=R_{\mathrm{n}}(t=0)
$$

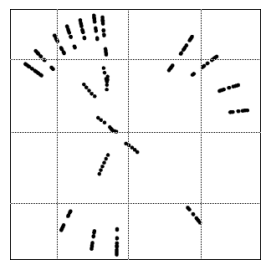




\subsection{Blasenpulsationen}

Eine frühe Arbeit zur Beschreibung der radialen Blasendynamik stammt von RAYLEigh [21]. Er leitet ein Modell für den sphärischen Kollaps einer leeren Blase aus der Energiebetrachtung der um die Blase bewegten inkompressiblen Flüssigkeit ab. Die Rayleigh'sche Betrachtung beschreibt eine nicht getriebene kollabierende Blase:

$$
R=R\left(R_{\mathrm{n}}, t\right)
$$

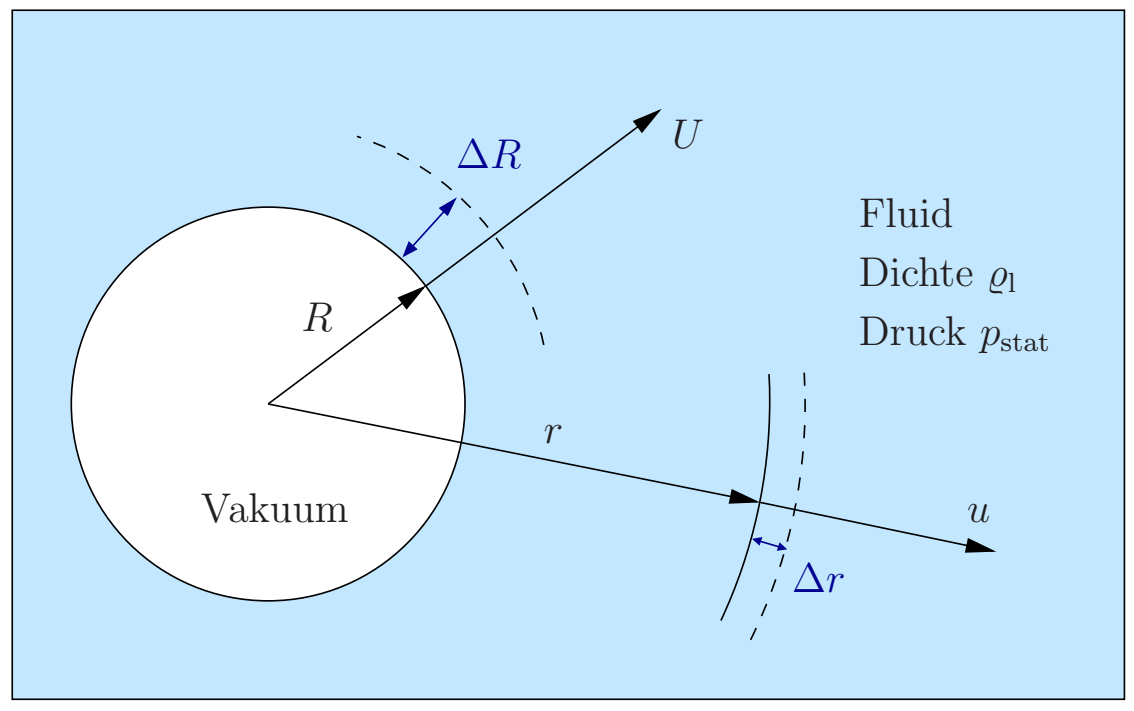

Abbildung 2.1: Betrachtung der sphärischen Oberflächenbewegung einer Vakuumblase in einem inkompressiblen Fluid nach Rayleigh.

Die kinetische Energie in dem die Blase umgebenden Fluid beträgt:

$$
E_{\text {kin }}=\frac{1}{2} \varrho_{1} \int_{R}^{\infty} 4 \pi r^{2} u^{2} \mathrm{~d} r
$$

$u$ Geschwindigkeit eines Fluidelements

$\varrho_{1} \quad$ konstante Dichte des Fluids (inkompressibel)

$U$ Blasenwandgeschwindigkeit

Mit dem sich aus der Kontinuitätsgleichung ableitenden Zusammenhang $u=$ $U R^{2} / r^{2}$ lässt sich das Integral ausführen und die kinetische Energie der Flüssigkeit angeben ${ }^{1}$ :

$$
E_{\text {kin }}=2 \pi \varrho_{1} U^{2} R^{3}
$$

1 wobei $u(r \rightarrow \infty)=0$ angenommen wird 
Die potentielle Energie, die die Blase durch ein fiktives Aufziehen gegen den als konstant angenommenen Außendruck des Fluids aufnimmt, beträgt:

$$
E_{\text {pot }}=\int_{R_{0}=0}^{R} p \mathrm{~d} V=p \int_{0}^{R} \mathrm{~d} V=p \frac{4 \pi}{3} R^{3}
$$

Werden Dämpfungsterme vernachlässigt, so ist die Summe von potentieller und kinetischer Energie eine Erhaltungsgröße:

$$
E_{\text {kin }}+E_{\text {pot }}=\text { const. }
$$

Im Umkehrpunkt einer Blasenschwingung $\left(R=R_{\max }\right)$ wird die kinetische Energie gerade Null. Damit lässt sich die Konstante bestimmen und mit (2.1) sowie (2.2) ergibt sich:

$$
2 \pi \varrho_{1} U^{2} R^{3}+\frac{4 \pi}{3} p R^{3}=\frac{4 \pi}{3} p R_{\max }^{3}
$$

Zeitliche Ableitung und Umstellungen der Terme führt auf die nichtlineare Differentialgleichung zur Beschreibung der radialen Blasenoszillation für inkompressible Flüssigkeiten, dem Rayleigh'schen Blasenmodell:

$$
R \ddot{R}+\frac{3}{2} \dot{R}^{2}=-\frac{1}{\varrho_{1}} \cdot p
$$

Im reinen Rayleigh'schen Modell wird nur ein statischer Umgebungsdruck angenommen, im Blaseninneren ist Vakuum:

$$
p=p_{\text {stat }}
$$

Die Annahme einer Vakuumblase bedeutet, dass sich kein Gas in der Blase befindet, das als rückstellende „Feder" wirken könnte, um ein Schwingungsverhalten zu bewirken. Das heißt die Blase wird einmalig kollabieren.

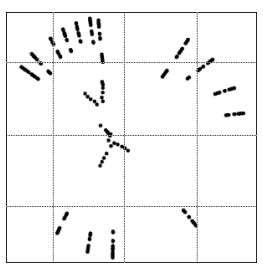




\subsubsection{Minnaert'sche Erweiterung}

Im Rayleigh'schen Modell wird zunächst nur ein äußerer, die Blase komprimierender Druck angenommen. Von Minnaert [22] wurde ein Blaseninnendruck eingeführt, der einen Gleichgewichtszustand (den endlichen „Ruheradius“) der Blase ermöglicht. Der Druckterm des Rayleigh-Modells (2.4) schreibt sich dann als Differenz von Außen- zu Innendruck:

$$
\begin{aligned}
p & =p_{\mathrm{a}}-p_{\mathrm{i}} \\
& =p_{\text {stat }}-p_{\text {stat }}\left(\frac{R_{\mathrm{n}}}{R}\right)^{3 \eta}
\end{aligned}
$$

$$
\begin{aligned}
p_{\mathrm{a}}=p(R) & \text { Umgebungsdruck im Fluid } \\
p_{\mathrm{i}} & \text { Gasdruck im Blaseninneren } \\
p_{\text {stat }} & \text { Umgebungsdruck im Fluid (statischer bzw. Luftdruck) } \\
\eta & \text { Polytropenexponent } \\
R_{\mathrm{n}} & \text { Gleichgewichtsradius der Blase }
\end{aligned}
$$

Der Innendruck wird durch Kompression eines idealen Gases mit dem Polytropenexponenten $\eta$ angesetzt.

Das sich so aus dem Rayleigh'schen Modell (2.4) und dem erweiterten Druckterm (2.5) zusammensetzende Rayleigh-Minnaert-Blasenmodell hat die Gestalt:

$$
R \ddot{R}+\frac{3}{2} \dot{R}^{2}=\frac{1}{\varrho_{\mathrm{l}}}\left(p_{\text {stat }}\left(\frac{R_{\mathrm{n}}}{R}\right)^{3 \eta}-p_{\text {stat }}\right)
$$

Die Linearisierung dieses Modells durch den Ansatz $R=R_{\mathrm{n}}+\tilde{R}$ führt auf ein Differentialgleichungssystem in der Form eines harmonischen Oszillators:

$$
\ddot{\tilde{R}}+\omega_{\text {res }}^{2} \tilde{R}=0 \quad \text { mit } \quad \omega_{\text {res }}^{2}=\frac{3 \eta p_{\text {stat }}}{\varrho_{1} R_{\mathrm{n}}^{2}}
$$

Die lineare Resonanzfrequenz des Modells lässt sich in dieser Form ablesen:

$$
\nu_{\text {res }}^{\operatorname{lin}}=\frac{1}{2 \pi R_{\mathrm{n}}} \sqrt{\frac{3 \eta p_{\text {stat }}}{\varrho_{\mathrm{l}}}}
$$

Der Polytropenexponent kann zwischen dem isothermen und adiabatischen Fall schwanken $\eta=1 \ldots \gamma$. Der Adiabatenexponent folgt abhängig von der Molekülspezies aus deren Anzahl an Freiheitsgraden $\gamma=(f+2) / f$. Unter Normalbedingungen $\Theta=20{ }^{\circ} \mathrm{C}$ und $p_{\text {stat }}=100 \mathrm{kPa}$ und mit der Dichte von Wasser $\left(\varrho_{1}=998 \mathrm{~kg} / \mathrm{m}^{3}\right)$ ergibt sich die einfache Abschätzung:

$$
\nu_{\mathrm{res}}^{\operatorname{lin}} R_{\mathrm{n}} \approx 3 \mathrm{~m} / \mathrm{s}
$$

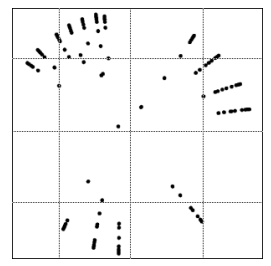




\subsubsection{Erweitertes Rayleigh-Modell}

Weitere Ergänzungen an dem Rayleigh'schen Blasenmodell folgten von PLESSET [23], der das Modell um einen Oberflächenspannungsterm ergänzte und von Poritsky [24], der die Viskosität einbezog:

$$
p=\frac{2 \sigma}{R}+\frac{4 \mu \dot{R}}{R}+p_{\text {stat }}+\tilde{p}(t)-\left(p_{\text {stat }}+\frac{2 \sigma}{R_{\mathrm{n}}}\right)\left(\frac{R_{\mathrm{n}}}{R}\right)^{3 \eta}
$$

$\sigma$ Oberflächenspannung

$\mu$ dynamische Viskosität der Flüssigkeit

$\tilde{p}(t) \quad$ eingekoppelter Schallwechseldruck

Hier ist außerdem schon ein Term für den eingekoppelten Schallwechseldruck enthalten.

Durch Druckschwankungen zwischen dem Gasdruck im Inneren der Blase und dem Umgebungsdruck im Fluid wird die Blase zu Schwingungen getrieben.

Der Druckterm (2.8) in die Rayleigh-Gleichung (2.4) eingesetzt führt auf:

$$
R \ddot{R}+\frac{3}{2} \dot{R}^{2}=\frac{1}{\varrho_{\mathrm{l}}} \cdot\left[\left(p_{\text {stat }}+\frac{2 \sigma}{R_{\mathrm{n}}}\right)\left(\frac{R_{\mathrm{n}}}{R}\right)^{3 \eta}-\frac{2 \sigma}{R}-\frac{4 \mu \dot{R}}{R}-p_{\text {stat }}-\tilde{p}(t)\right]
$$

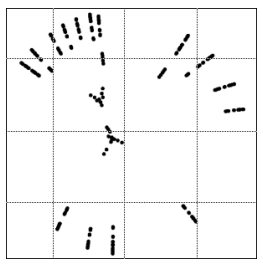




\subsubsection{Keller-Miksis-Modell}

Auf der Arbeit von KeLleR und Kolodner [25] basierend berücksichtigen KeLLER und Miksis [26] die Schallabstrahlung in einem schwach kompressiblen Fluid. Unter der Annahme einer rein sphärischen Oszillation und einer konstanten Anzahl an Gasmolekülen in der Blase ergibt sich die Keller-Miksis-Gleichung:

$$
\left(1-\frac{\dot{R}}{C_{\mathrm{l}}}\right) R \ddot{R}+\frac{3}{2}\left(1-\frac{\dot{R}}{3 C_{\mathrm{l}}}\right) \dot{R}^{2}=\left(1+\frac{\dot{R}}{C_{\mathrm{l}}}\right) \frac{p}{\varrho_{\mathrm{l}}}+\frac{R}{\varrho_{\mathrm{l}} C_{\mathrm{l}}} \frac{\mathrm{d} p}{\mathrm{~d} t}
$$

$$
\begin{aligned}
\dot{R}=U(t) & \text { Geschwindigkeit der Blasenwand } \\
C_{1} & \text { Schallgeschwindigkeit in der Flüssigkeit }
\end{aligned}
$$

Getrieben wird die Blase durch die Differenz zwischen innerem und äußerem Druck $p=p_{\mathrm{i}}-p_{\mathrm{a}}$, nach LAUTERBorN et al. [27] ergänzt um den Dampfdruck und eine sinusförmige Schallanregung:

$$
\begin{aligned}
& p=\left(p_{\text {stat }}-p_{\mathrm{v}}+\frac{2 \sigma}{R_{\mathrm{n}}}\right)\left(\frac{R_{\mathrm{n}}}{R}\right)^{3 \eta}-p_{\text {stat }}+p_{\mathrm{v}}-\frac{2 \sigma}{R}-\frac{4 \mu}{R} \dot{R}-\tilde{p} \\
& \tilde{p}=p_{\mathrm{A}} \sin (2 \pi \nu t)
\end{aligned}
$$

$p_{\mathrm{v}} \quad$ Dampfdruck über dem Fluid

$\nu$ Anregungsfrequenz

Das Keller-Miksis-Modell liefert eine recht genaue Beschreibung der Blasendynamik bei relativ geringem Rechenaufwand. Daher ist allen folgenden Betrachtungen das nichtlineare Keller-Miksis-Modell zugrunde gelegt.

Die Linearisierung des Keller-Miksis-Modells mit dem Ansatz $R(t)=R_{\mathrm{n}}+\tilde{R}(t)$ wird zum Beispiel angegeben in PARLITz et al. [16] in der Form eines gedämpften, getriebenen harmonischen Oszillators:

$$
\ddot{\tilde{R}}+\delta \dot{\tilde{R}}+\omega_{\text {res }}^{2} \tilde{R}=-\frac{1}{\varrho_{1} R_{\mathrm{n}}} \tilde{p}
$$

Der Dämpfungsterm $\delta$ und die lineare Resonanzfrequenz $\omega_{\text {res }}$ des Systems sind dabei:

$$
\begin{aligned}
\delta & =\frac{4 \mu}{\varrho_{1} R_{\mathrm{n}}^{2}}+\frac{\omega_{\mathrm{res}}^{2} R_{\mathrm{n}}}{C_{\mathrm{l}}} \\
\omega_{\text {res }}^{2} & =\frac{1}{\varrho_{\mathrm{l}} R_{\mathrm{n}}^{2}}\left(3 \eta\left(p_{\text {stat }}+\frac{2 \sigma}{R_{\mathrm{n}}}\right)-\frac{2 \sigma}{R_{\mathrm{n}}}\right)
\end{aligned}
$$

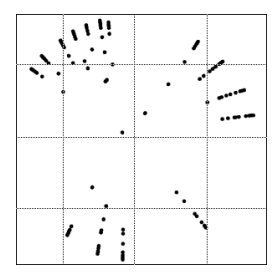


Die Lösung des harmonischen Oszillators ergibt sich über den Ansatz:

$$
\begin{aligned}
& \tilde{R}=R_{\mathrm{A}} \mathrm{e}^{\imath \omega t}, \quad \tilde{p}(t)=p_{\mathrm{A}} \mathrm{e}^{\imath \omega t} \quad \text { mit } \quad R_{\mathrm{A}} \in \mathbb{C}, \quad p_{\mathrm{A}} \in \mathbb{R} \\
\Rightarrow \quad & -\omega^{2} R_{\mathrm{A}} \mathrm{e}^{\imath \omega t}+\imath \omega \delta R_{\mathrm{A}} \mathrm{e}^{\imath \omega t}+\omega_{\text {res }}^{2} R_{\mathrm{A}} \mathrm{e}^{\imath \omega t}=-\frac{1}{\varrho_{\mathrm{l}} R_{\mathrm{n}}} p_{\mathrm{A}} \mathrm{e}^{\imath \omega t} \\
\Rightarrow & R_{\mathrm{A}}=\frac{p_{\mathrm{A}}}{\varrho_{\mathrm{l}} R_{\mathrm{n}}}\left(\frac{1}{\omega^{2}-\omega_{\text {res }}^{2}-\imath \omega \delta}\right)
\end{aligned}
$$

Als Lösung der linearisierten Keller-Miksis-Gleichung für die Radiusauslenkungen $\tilde{R}$ über den Ruheradius $R_{\mathrm{n}}$ der Blase resultiert:

$$
\tilde{R}(t)=\frac{\tilde{p}(t)}{\varrho_{1} R_{\mathrm{n}}}\left(\frac{1}{\omega^{2}-\omega_{\mathrm{res}}^{2}-\imath \omega \delta}\right)
$$

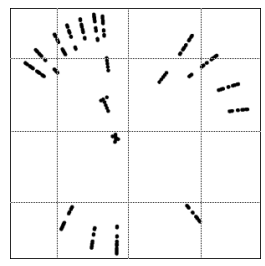




\subsubsection{Typische Blasenschwingungen}

Einige Lösungen der Keller-Miksis-Gleichung (2.10) sind in Abbildung 2.2 für verschiedene Schalldruckamplituden dargestellt. Die betrachtete Blase mit einem Ruheradius von $R_{\mathrm{n}}=5 \mu \mathrm{m}$ wird zeitharmonisch bei $\nu=20 \mathrm{kHz}$ getrieben. Nach Metтin et al. [28] ist ihre Größe typisch für die in akustischen Kavitationsstrukturen dieses Frequenzbereiches auftretenden Blasen.

Eine schwach getriebene Blase $\left(p_{\mathrm{A}}=80 \mathrm{kPa}\right)$ folgt im Wesentlichen dem Druckverlauf des treibenden Schallfeldes, wenngleich nichtharmonische Anteile auftreten, wie in Abbildung $2.2 \mathrm{zu}$ sehen ist. Die Blase erreicht in diesem Fall ihren maximalen Radius zu der Phasenlage des maximalen Unterdrucks. Der Oszillationsverlauf für einen höheren Anregungsdruck $\left(p_{\mathrm{A}}=120 \mathrm{kPa}\right)$ zeigt ein deutlich nichtlineares Verhalten. Die Blase wird fast bis auf das 6fache ihres Ruheradius aufgezogen, erreicht dieses Maximum phasenverschoben zum Druckminimum und führt nach ihrer Expansionsphase eine Reihe von Nachschwingungen um ihren Ruheradius aus. Genaugenommen kommt sie unterhalb ihres Ruheradius zur Ruhe, da sie durch den Überdruck komprimiert wird. Die unterste Auftragung in Abbildung 2.2 stellt eine noch stärker getriebene Blase dar $\left(p_{\mathrm{A}}=160 \mathrm{kPa}\right)$. Die Expansionsphase dieser Blase reicht deutlich in die Überdruckphase hinein. Die Blase erreicht dabei fast das 16fache ihres Ruheradius und vollführt einen starken Kollaps.

Mit abnehmender Frequenz des treibenden Schallfeldes wird die Unterdruckphase länger, wodurch die Blasen zu größeren Radien aufgezogen werden und heftigere Kollapse durchlaufen. Umgekehrt werden mit höheren Frequenzen die höherperiodischen Schwingungsformen verstärkt, da mit der verkürzten Unterdruckphase die Blase nicht vollständig ausschwingen kann, bevor sie wieder aufgezogen wird (Abbildung 2.3).

Getriebene nichtlineare Oszillatoren können ein komplexes Schwingungsverhalten zeigen (NAYFEH und Mook [29]). Dies ist auch der Fall für getriebene Blasen, wie zum Beispiel von PARLitz et al. [30] oder Akhatov et al. [31] beschrieben. Bei den hier relevanten Parameterbereichen gilt, dass höherperiodische Schwingungsformen bis hin zum chaotischen Zustand vor allem mit zunehmender Schalldruckamplitude und anwachsendem Ruheradius der Blasen beobachtet werden. Kleineren nur schwach getriebenen Blasen lässt sich dagegen gut die Schallfeldperiode aufprägen. Abbildung 2.4 zeigt die Periodizitäten der Keller-Miksis-Gleichung in der Parameterebene von Schalldruckamplitude und Ruheradius bei $\nu=20 \mathrm{kHz}$ Anregungsfrequenz. Unterhalb einer Anregungsamplitude von etwa $50 \mathrm{kPa}$ und

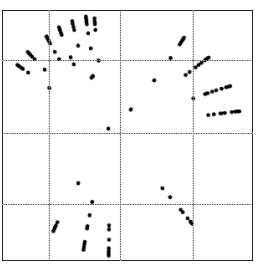


einem Ruheradius von etwa $10 \mu \mathrm{m}$ treten fast ausschließlich einfach periodische Pulsationen auf.

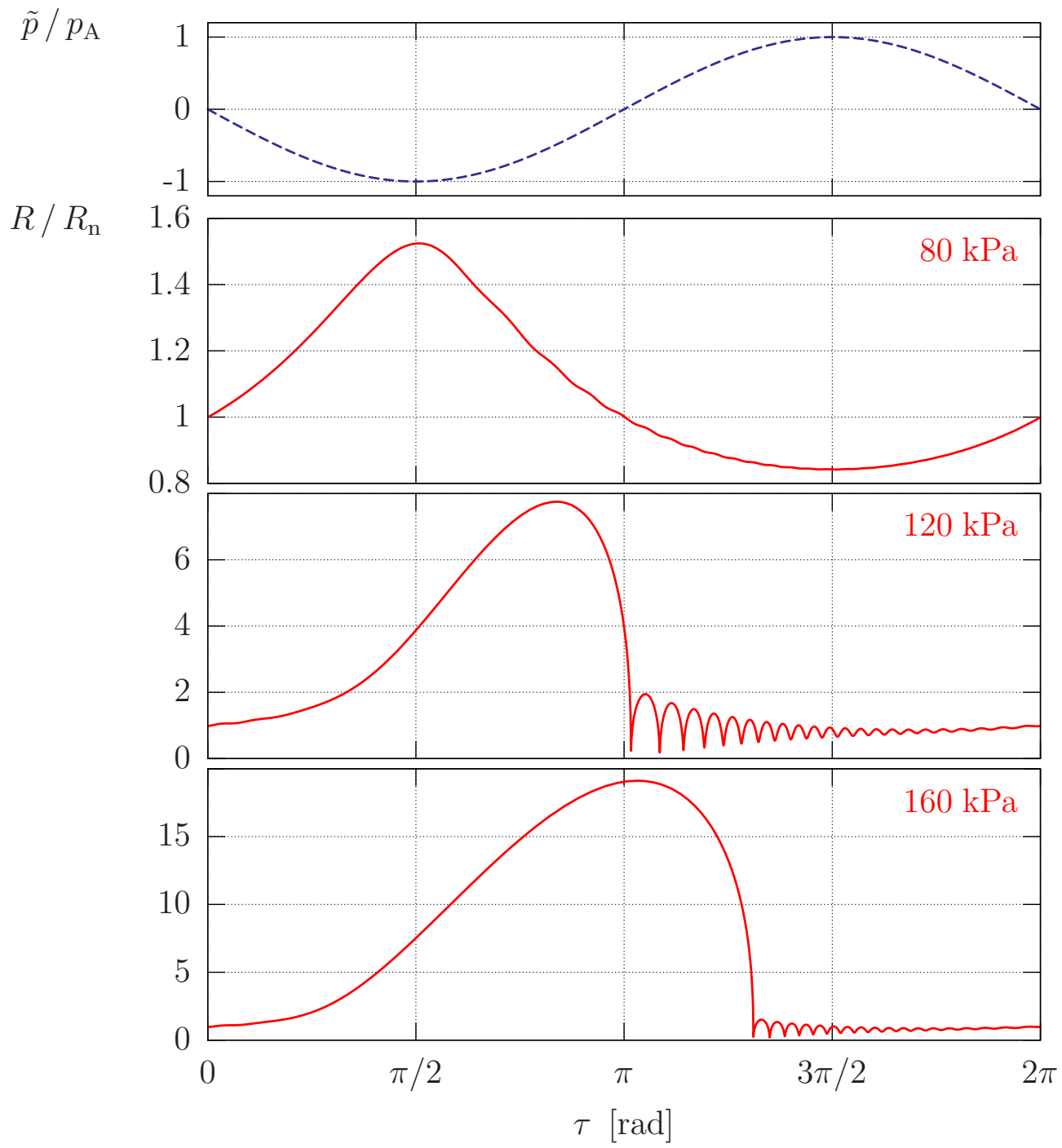

Abbildung 2.2: Zeitlicher Druckverlauf und radiale Blasenpulsation nach Keller und Miksis (2.10) für verschiedene Schalldruckamplituden. Gezeigt ist der Radiusverlauf während einer Anregungsperiode nach Einschwingen des Systems. Aufgetragen gegen die entdimensionalisierte Zeitachse $\tau=\omega t ; R_{\mathrm{n}}=5 \mu \mathrm{m}, \nu=20 \mathrm{kHz}, p_{\mathrm{A}}=80,120,160 \mathrm{kPa}$.

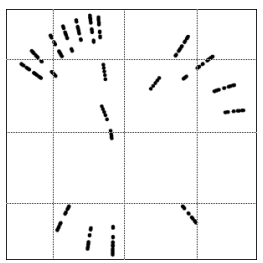




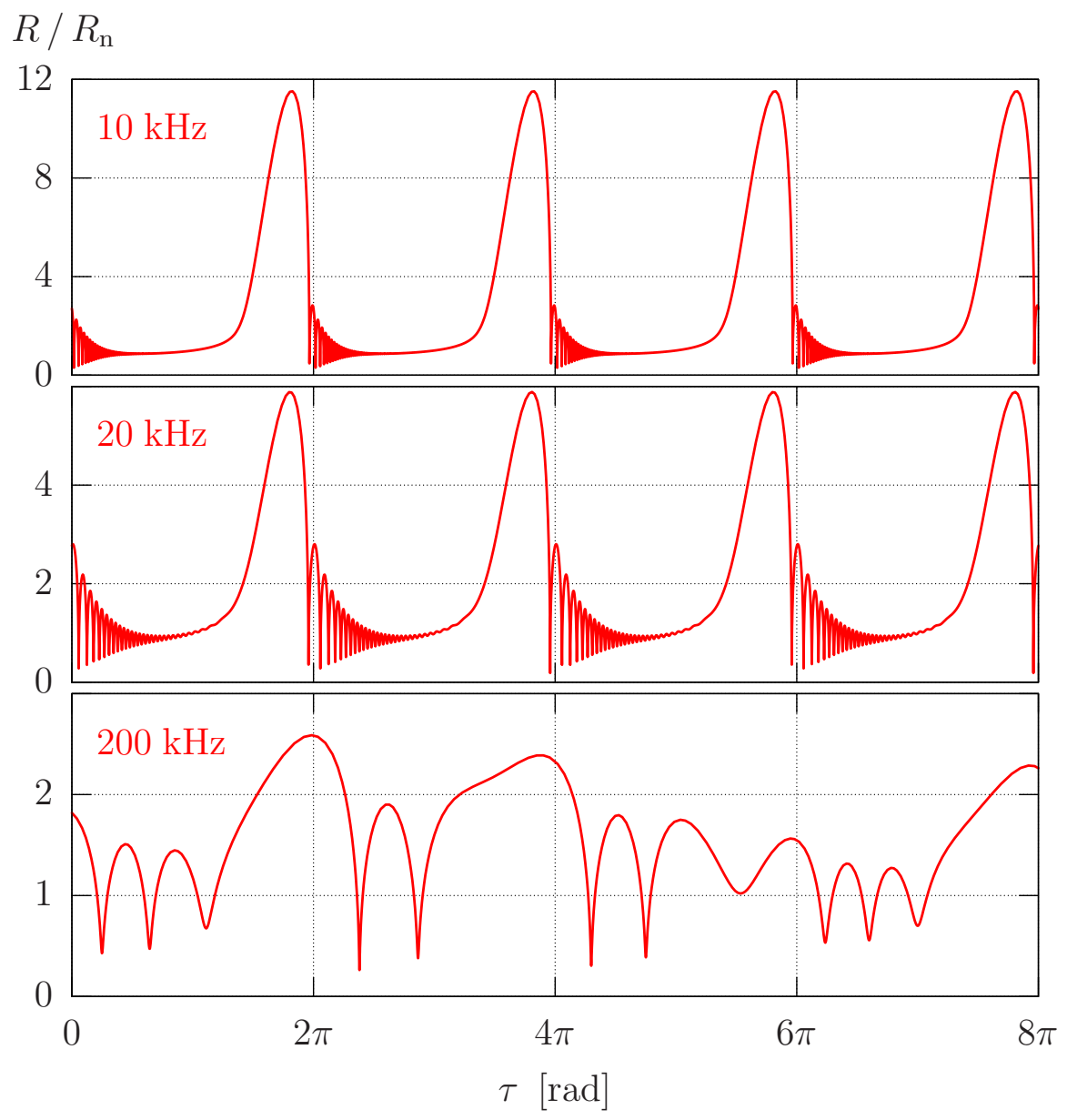

Abbildung 2.3: Radiale Blasenpulsation nach dem Keller-Miksis-Modell (2.10) für verschiedene Frequenzen des harmonisch treibenden Schallfeldes; $R_{\mathrm{n}}=5 \mu \mathrm{m}, p_{\mathrm{A}}=120 \mathrm{kPa}, \nu=10,20,200 \mathrm{kHz}$.

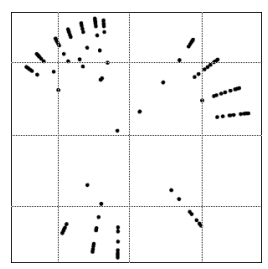




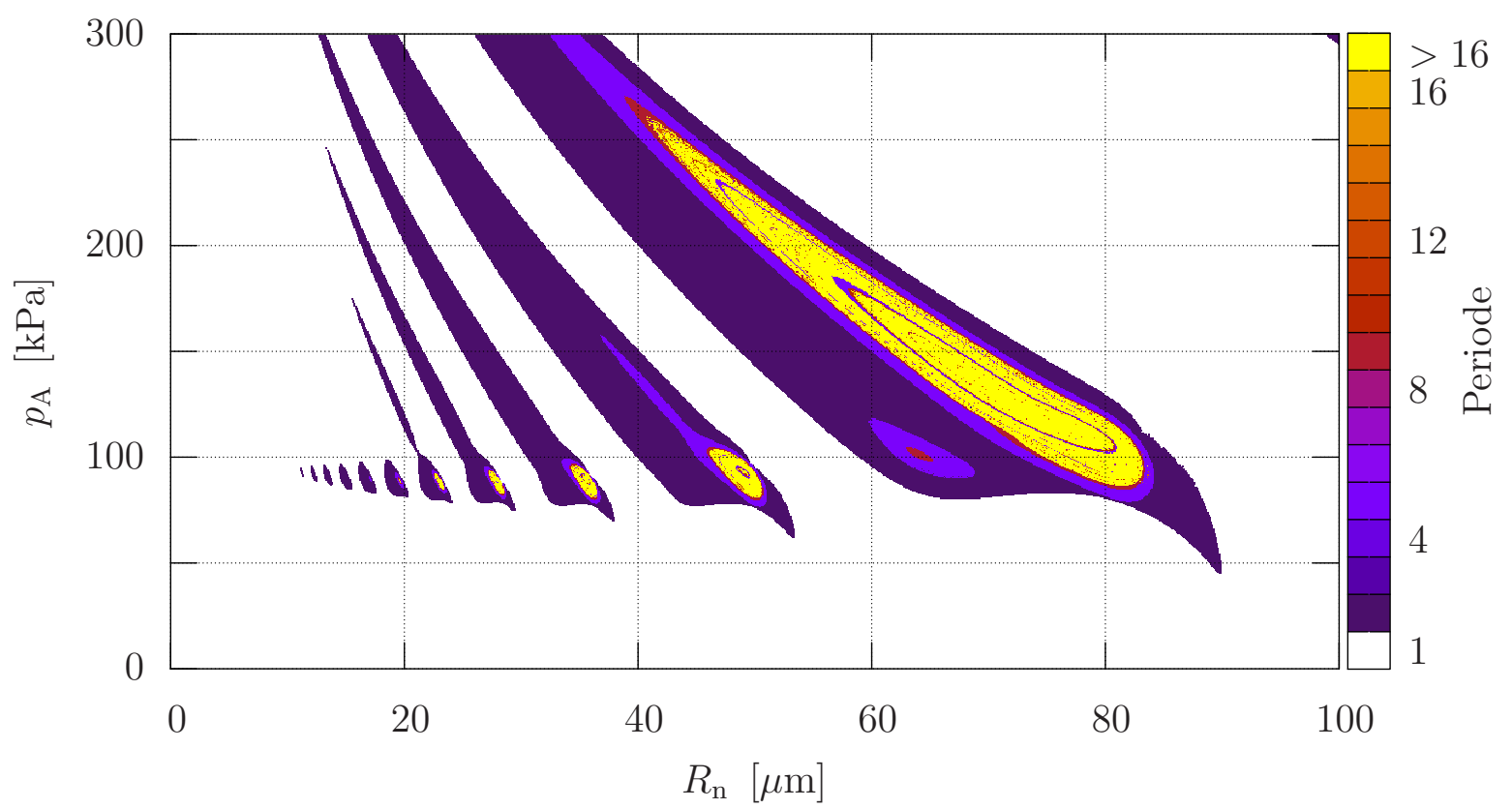

Abbildung 2.4: Periodizität der Blasenpulsationen in Perioden des Schallfeldes dargestellt im Parameterraum von Schalldruckamplitude gegen Ruheradius. Als nicht periodisch bzw. höherperiodisch (Periode $>16$ ) werden die Lösungen der Keller-Miksis-Gleichung (2.10) zu Parameterpunkten eingestuft, zu denen sich nach 16 Perioden keine wiederkehrende Schwingungsform einstellt. Zuvor wird der Einschwingvorgang über 256 Perioden abgewartet; Anfangsbedingungen: $(R(0), U(0))=\left(R_{\mathrm{n}}, 0\right)$; $\nu=20 \mathrm{kHz}, \eta=1$.

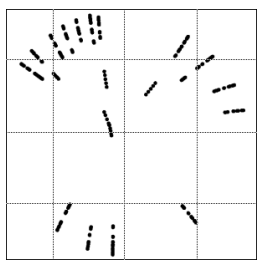




\subsection{Blake-Schwelle}

Dieser Abschnitt beschäftigt sich mit der Blake-Schwelle, wie sie beispielsweise von Young [6] abgeleitet wird. Nach der formalen Darstellung wird ihre Bedeutung in dem in dieser Arbeit weiter betrachteten Parameterbereich diskutiert.

Eine sphärische Gasblase befindet sich im Gleichgewicht, solange die äußeren Druckgrößen gerade dem Innendruck entsprechen. Daraus folgt wie in Abbildung 2.5 verdeutlicht unter Normaldruck die Gleichgewichtsbedingung:

$$
p_{\mathrm{i}}=p_{\text {stat }}+\frac{2 \sigma}{R_{\mathrm{n}}}
$$
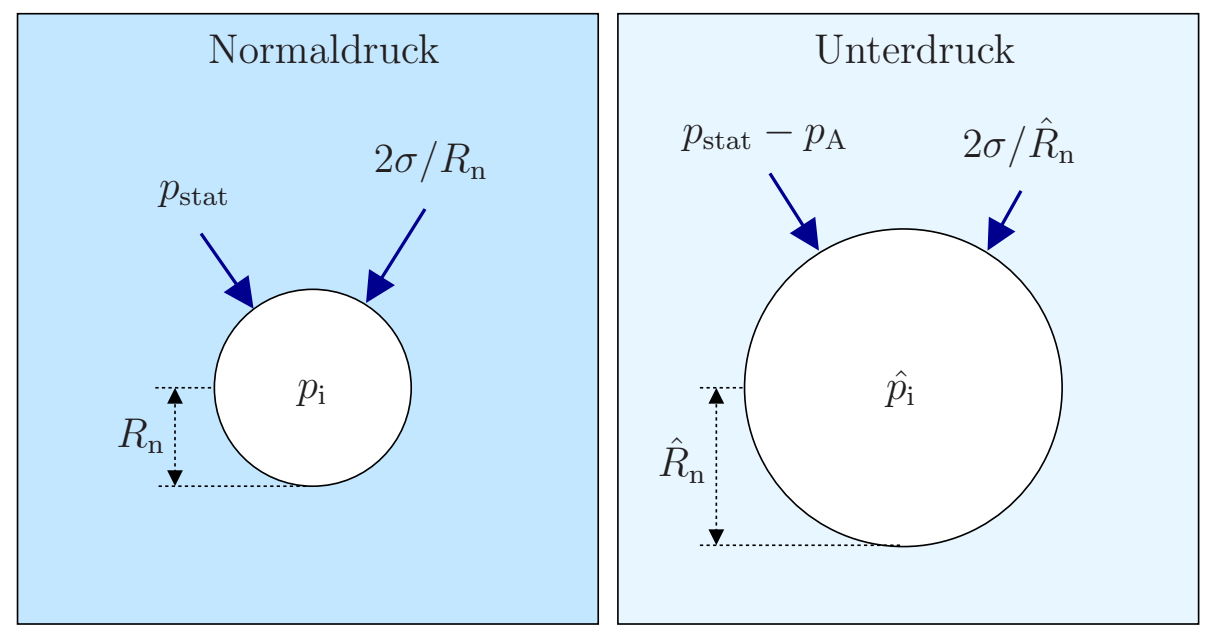

Abbildung 2.5: Eine Kavitationsblase bei Normaldruck im Gleichgewicht und bei Unterdruck; im Unterdruckbereich ist der statische Druck um $p_{\mathrm{A}}$ reduziert, die Blase vergrößert sich, damit verringert sich der Innendruck $\hat{p}_{\mathrm{i}}<p_{\mathrm{i}}$ und gleichzeitig wird der Oberflächenspannungsterm schwächer.

Wird die Blase einem Unterdruck der konstanten Amplitude $p_{\mathrm{A}}$ ausgesetzt, so vergrößert sich ihr Radius, und der Gasdruck im Inneren fällt ab gemäß $p_{\mathrm{i}} V_{\mathrm{n}}=$ $\hat{p}_{\mathrm{i}} \hat{V}_{\mathrm{n}}$ (isotherm). Im neuen Gleichgewicht muss gelten:

$$
\begin{gathered}
\hat{p}_{\mathrm{i}}=p_{\mathrm{i}}\left(\frac{R_{\mathrm{n}}}{\hat{R}_{\mathrm{n}}}\right)^{3}=p_{\text {stat }}-p_{\mathrm{A}}+\frac{2 \sigma}{\hat{R}_{\mathrm{n}}} \\
\Rightarrow \quad p_{\mathrm{A}}=p_{\text {stat }}+\frac{2 \sigma}{\hat{R}_{\mathrm{n}}}-\left(p_{\text {stat }}+\frac{2 \sigma}{R_{\mathrm{n}}}\right)\left(\frac{R_{\mathrm{n}}}{\hat{R}_{\mathrm{n}}}\right)^{3}
\end{gathered}
$$

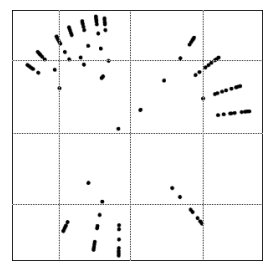


Ausgedrückt ist somit die Abhängigkeit der Schalldruckamplitude von dem neuen sich einstellenden Gleichgewichtsradius $p_{\mathrm{A}}=p_{\mathrm{A}}\left(\hat{R}_{\mathrm{n}}\right)$. Der sogenannte kritische Radius ist gerade im Minimum des Schalldruckverlaufs erreicht:

$$
\begin{aligned}
\frac{\partial p_{\mathrm{A}}}{\partial \hat{R}_{\mathrm{n}}} & \stackrel{!}{=} 0 \\
& =-\frac{2 \sigma}{\hat{R}_{\mathrm{n}}^{2}}+3\left(p_{\text {stat }}+\frac{2 \sigma}{R_{\mathrm{n}}}\right) \frac{R_{\mathrm{n}}^{3}}{\hat{R}_{\mathrm{n}}^{4}} \\
\Rightarrow \quad R_{\text {krit. }} & =\left(\frac{3\left(p_{\text {stat }}+2 \sigma / R_{\mathrm{n}}\right) R_{\mathrm{n}}^{3}}{2 \sigma}\right)^{1 / 2}
\end{aligned}
$$

Wird dieser kritische Radius überschritten $\left(\hat{R}_{\mathrm{n}}>R_{\text {krit. }}\right)$, so sinkt die Oberflächenspannung zu stark ab und kann den Innendruck nicht mehr ausgleichen. Das heißt es gibt keine neue Gleichgewichtslage von $\hat{R}_{\mathrm{n}}$ für $p_{\mathrm{A}}$, und die Blase expandiert ungehindert immer weiter.

Wird der kritische Radius (2.15) in den Ausdruck der Schallamplitude (2.14) eingesetzt, ergibt sich der auf BLAKE [32] zurückgehende Grenzwert des statischen Druckes, der notwendig ist, um die Gasblase vom Gleichgewichtsradius gerade auf den kritischen Radius aufzuziehen. Da ein zeitlich konstanter Unterdruck betrachtet wird, handelt es sich um die sogenannte statische Blake-Schwelle:

$$
p_{\text {Blake }}=p_{\text {stat }}+\frac{4 \sigma}{3}\left(\frac{2 \sigma}{3 R_{\mathrm{n}}^{3}\left(p_{\text {stat }}+2 \sigma / R_{\mathrm{n}}\right)}\right)^{1 / 2}
$$

Im statischen Fall $(\nu=0)$ würde eine Blase, die einmal die instabile Schwelle überschritten hat, bis ins Unendliche weiter anwachsen. Dagegen erreicht eine periodisch getriebene Blase einen eingeschwungenen Zustand, in dem sie stark nichtlineare Oszillationen ausführt. Dieser Übergang von einer schwach zu einer stark oszillierenden Kavitationsblase wird als dynamische Blake-Schwelle bezeichnet. Die dynamische Blake-Schwelle ist in der Auftragung 2.6 von $R_{\max }$, dem maximalen Radius während einer Periode, gegen den Ruheradius $R_{\mathrm{n}}$ zu sehen. Es zeichnet sich ein deutliches sprunghaftes Anwachsen des maximalen Radius der Blase für einen bestimmten Ruheradius abhängig von der anregenden Druckamplitude $p_{\mathrm{A}}$ ab. Als Kriterium für das Erreichen dieser Blake-Schwelle wird angenommen, dass die Blase ein Vielfaches $\Omega$ ihres Ruheradius erreicht:

$$
R_{\max } \geq \Omega \cdot R_{\mathrm{n}}
$$

Der Faktor $\Omega$ wird oft im Bereich $\Omega=2 \ldots 3$ gewählt, um eine deutliche nichtlineare Blasendynamik zu kennzeichnen. Um einen heftigen Blasenkollaps anzuzeigen, können auch Werte um 5...10 angesetzt werden.

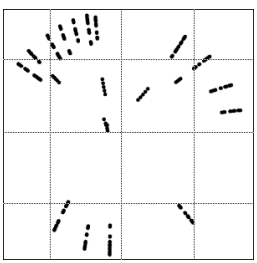




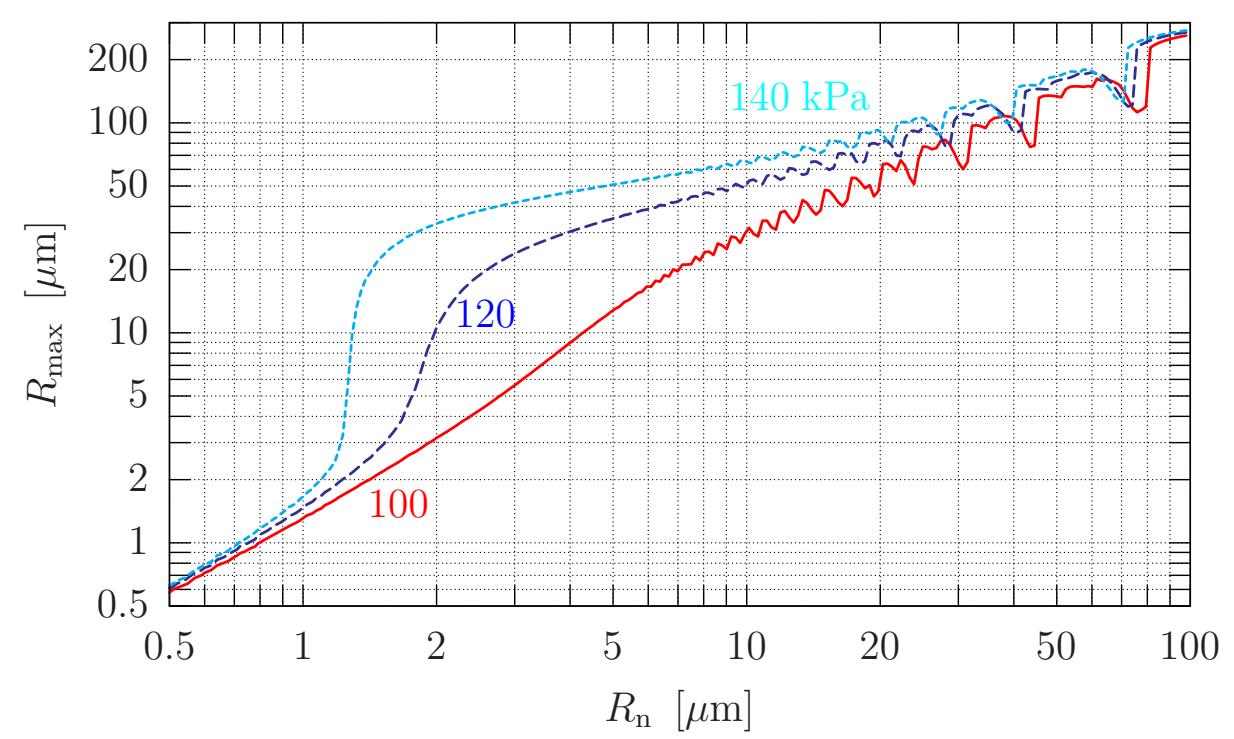

Abbildung 2.6: Doppeltlogarithmische Auftragung des maximalen Radius eines Schwingungszyklus gegen den Ruheradius; die dynamische Blake-Schwelle ist im sprunghaften Anstieg deutlich zu erkennen, mit steigender Druckamplitude verschiebt sie sich zu kleineren Ruheradien; $\nu=20 \mathrm{kHz}, p_{\mathrm{A}}=100,120,140 \mathrm{kPa}, \eta=1$.

Mit zunehmender Anregungsdruckamplitude verlagert sich die Schwelle des sprunghaften Anstiegs leicht in Richtung kleinerer Ruheradien und der Sprung im Verlauf des Maximalradius wird steiler.

Die zu größeren Ruheradien hin beobachteten Maxima und Minima der Maximalradien in Auftragung 2.6 beruhen auf den nichtlinearen Resonanzen der Blase wie von LAUTERBorn et al. [33] beschrieben. Durch nichtlineare Effekte werden Vielfache sowie gebrochen Vielfache der Resonanzfrequenzen verstärkt, wie in Abbildung $2.7 \mathrm{zu}$ sehen.

Die Betrachtung der Blake-Schwelle führt zu der in Abbildung 2.8 gezeigten Abgrenzung im $p_{\mathrm{A}}-R_{\mathrm{n}}$-Parameterraum. Blasen, die auf ein Vielfaches ihres Ruheradius aufschwingen und anschließend entsprechend stark kollabieren, werden durch die dynamische Blake-Schwelle getrennt von jenen, die nur schwach expandieren.

Eine genauere Diskussion der Dynamik getriebener Blasen wird von PARLITz et al. [30] und LAUTERBORN et al. [33] geführt.

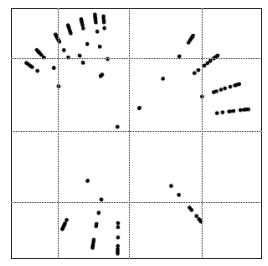




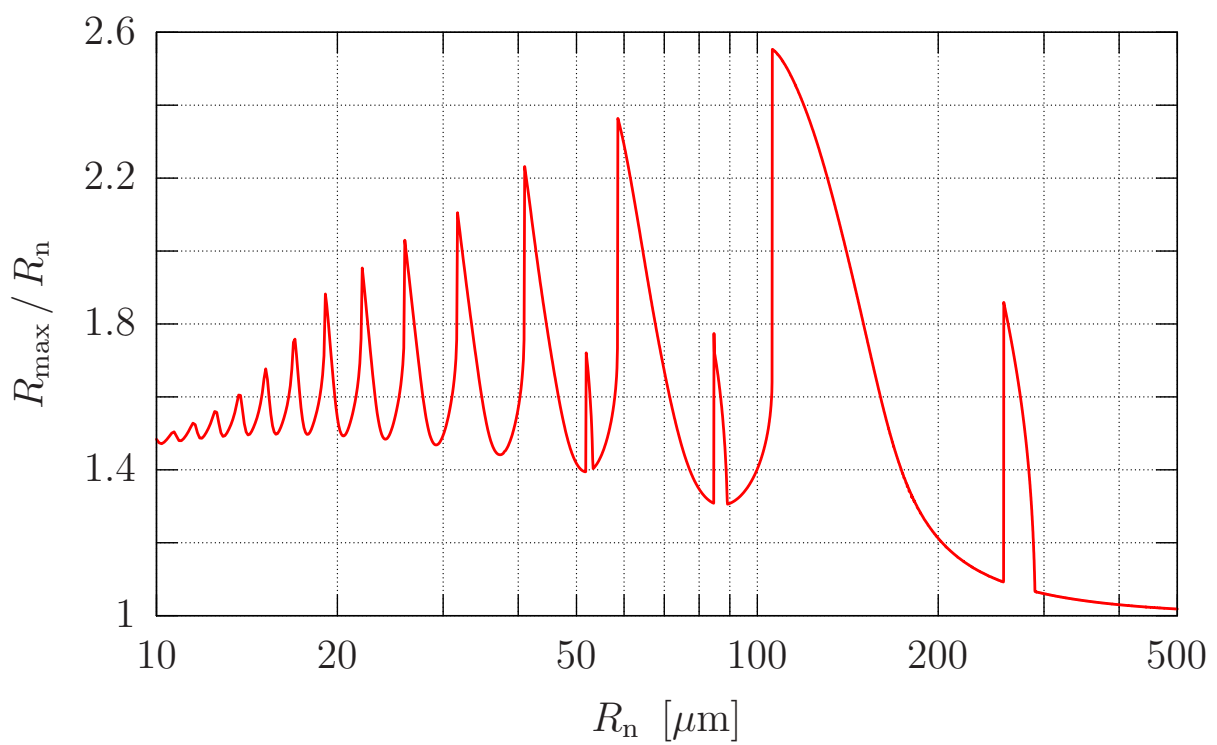

Abbildung 2.7: Resonanzantwort für Kavitationsblasen unterschiedlicher Ruheradien bei fester Frequenz; zu beachten ist die logarithmische Darstellung der $R_{\mathrm{n}}$-Achse; $\nu=20 \mathrm{kHz}, p_{\mathrm{A}}=70 \mathrm{kPa}, \eta=1$.

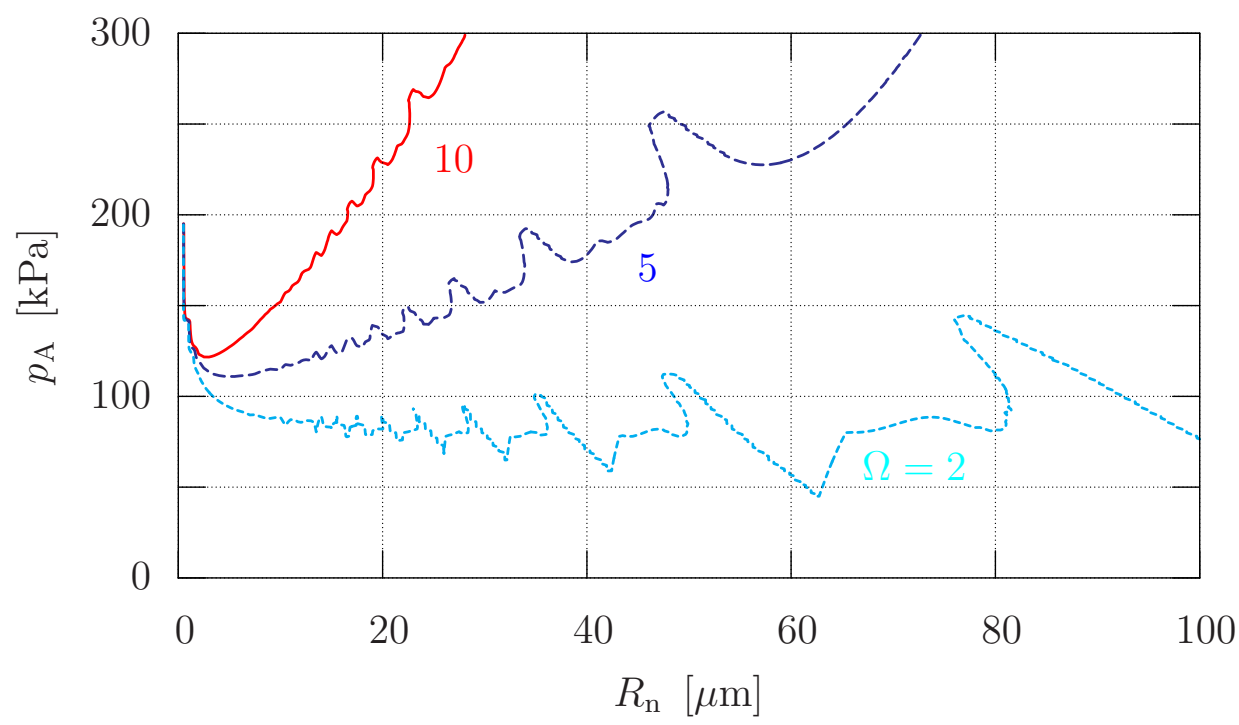

Abbildung 2.8: Darstellung der dynamischen Blake-Schwelle in der Parameterebene für Druckamplitude und Ruheradius. Die Konturlinien zeigen an, zu welchen Parametern eine Blase gerade das $\Omega$-fache ihres $R u-$ heradius erreicht. Darüberliegende Parameterwerte führen zu noch größerer Expansion; $\nu=20 \mathrm{kHz}, \Omega=2,5,10, \eta=1$.

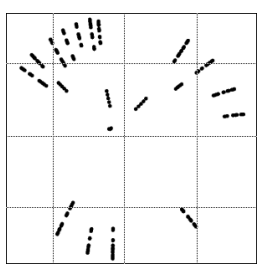




\subsection{Polytropenexponent}

Durch den Polytropenexponenten wird die Art des thermischen Prozesses charakterisiert, den die Blase während ihrer Pulsation durchläuft. Eine langsam ablaufende Blasenoszillation kann als isotherm $(\eta=1)$ angesehen werden, da ein ständiger Temperaturausgleich mit der Flüssigkeit möglich ist. Wird dagegen die Blase mit höheren Frequenzen getrieben oder kommt es auf sehr kurzen Zeitskalen zu einem heftigen Kollaps, so ist ein Temperaturausgleich nicht mehr möglich. Im Grenzfall des adiabatischen Prozesses $(\eta=\gamma)$ kommt es zu keinem Energieaustausch mit der Flüssigkeit, stattdessen wird das Gas in der Blase aufgeheizt. Während einer Oszillationsphase kann die Blase Bereiche zwischen diesen beiden Extrema durchlaufen:

$$
\eta(t)=1 \ldots \gamma
$$

$\eta$ Polytropenexponent

$\gamma$ Adiabatenexponent

Welcher Wert für den Polytropenexponenten $\eta$ anzusetzen ist, ist im Allgemeinen nicht einfach zu klären.

Bei einem adiabatischen Prozess wird die Energie der Blase in den möglichen translatorischen und oszillatorischen Freiheitsgraden der Molekülbewegungen im Blaseninneren gespeichert, es gilt:

$$
\gamma=\frac{f+2}{f} \equiv \frac{c_{\mathrm{p}}}{c_{\mathrm{V}}}
$$

$f \quad$ Freiheitsgrade der Gasmoleküle

$c_{\mathrm{p}}$ spezifische Wärme des Gases bei konstantem Druck

$c_{\mathrm{V}}$ spezifische Wärme des Gases bei konstantem Volumen

Falls es sich bei dem in der Blase befindlichen Gas um Luft handelt, so besagt zum Beispiel die von LoHse et al. [34, 35] formulierte Argon-Hypothese, dass alle „reaktionsfreudigen“ Moleküle $\left(\mathrm{O}_{2}, \mathrm{~N}_{2}\right)$ innerhalb der Blase nach hinreichend vielen Oszillationen zu Reaktionen angeregt werden und deren Produkte in die Flüssigkeit diffundieren, wodurch lediglich reaktionsträge Edelgase (überwiegend Argon) in der Blase verbleiben.

Die bindungslosen Edelgase besitzen lediglich die drei translatorischen Freiheitsgrade, womit in diesem Fall der Adiabatenexponent gerade $\gamma=5 / 3$ beträgt.

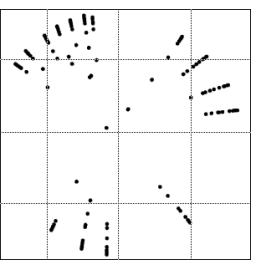


Der richtige Polytropenexponent ist zwar abhängig von den thermischen Eigenschaften des Fluids und des Gases, aber auch wesentlich von der Zeitskala bestimmt, auf der die Blasendynamik abläuft. Diese Zeitskala entspricht im Fall schwach getriebener Blasen gerade der Frequenz des antreibenden Schallfeldes:

$$
\eta=\eta(\omega)
$$

$\omega$ Kreisfrequenz des Schallfeldes

Um den Verlauf des Polytropenexponenten zwischen den Grenzfällen der isotherm und adiabatisch ablaufenden Blasenpulsationen angeben zu können, muss die thermische Dämpfung der Blase bestimmt werden.

Durch die Betrachtung der linearisierten Blasendynamik leitet Devin [36] einen Dämpfungsterm ab, der in drei Anteile aufspaltet: thermische Dämpfung durch Wärmeaustausch zwischen Gas in der Blase und dem umgebenden Fluid; akustische Dämpfung durch Schallabstrahlung in das Fluid und viskose Dämpfung durch die Viskosität an der Gas-Fluid Grenzschicht:

$$
\begin{aligned}
\delta_{\mathrm{th}} & =3(\gamma-1) \frac{\xi(\sinh (\xi)+\sin (\xi))-2(\cosh (\xi)-\cos (\xi))}{\xi^{2}(\cosh (\xi)-\cos (\xi))+3(\gamma-1) \xi(\sinh (\xi)-\sin (\xi))} \\
\delta_{\mathrm{ak}} & =\frac{\varrho_{1} R_{\mathrm{n}}^{3} \omega^{3}}{3 \eta p_{\mathrm{stat}} C_{\mathrm{l}}} \\
\delta_{\mathrm{vi}} & =\frac{4 \omega \mu}{3 \eta p_{\mathrm{stat}}}
\end{aligned}
$$

$\varrho_{1} \quad$ Dichte des Fluids

$R_{\mathrm{n}} \quad$ Ruheradius

$p_{\text {stat }}$ statischer Umgebungsdruck im Fluid

$C_{1} \quad$ Schallgeschwindigkeit im Fluid

$\mu$ Viskosität des Fluids

Von Devin [36] sowie von Eller [37] und Prosperetti [38, 39] wird der Polytropenexponent für lineare Blasenschwingungen angegeben:

$$
\eta=\frac{\gamma}{1+\delta_{\mathrm{th}}^{2}}\left(1+3 \frac{\gamma-1}{\xi} \frac{\sinh (\xi)-\sin (\xi)}{\cosh (\xi)-\cos (\xi)}\right)^{-1}
$$

Die Größe $\xi$ fasst die Abhängigkeit von den thermischen Eigenschaften, der Schallfeldfrequenz und dem Ruheradius zusammen:

$$
\xi=R_{\mathrm{n}} \sqrt{\frac{2 \omega}{D_{\mathrm{th}}}}
$$

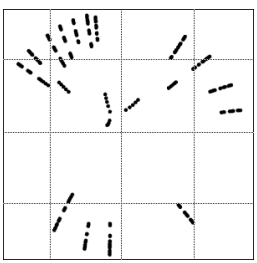




$$
\begin{aligned}
D_{\mathrm{th}}=\varkappa /\left(\varrho_{\mathrm{g}} c_{\mathrm{p}}\right) & \text { thermisches Diffusionsvermögen } \\
\varrho_{\mathrm{g}} & \text { Dichte des Gases in der Blase } \\
\varkappa & \text { Wärmeleitfähigkeit des Gases }
\end{aligned}
$$

Der Term $\sqrt{D_{\text {th }} / \omega}$ beschreibt die Eindringtiefe des thermischen Effekts in die Blase pro Schwingungszyklus. Eine genaue Beschreibung des thermischen Diffusionsvermögens wird von Prosperetti [38] gegeben. Somit ist die physikalische Bedeutung des Ausdrucks für $\xi$ zu verstehen als Verhältnis von Ruheradius der Blase zur thermischen Eindringtiefe, bezogen auf die Zeitskala der Blasendynamik.

In Abbildung 2.9 ist der Polytropenexponent nach (2.16) gegen die Kreisfrequenz für verschiedene typische Ruheradien aufgetragen. Für einen festen Ruheradius kommt es erst ab einer bestimmten Grenzfrequenz zu einer Änderung im Polytropenexponenten. Mit anwachsendem Radius verschiebt sich diese Grenze hin zu niedrigeren Frequenzen.

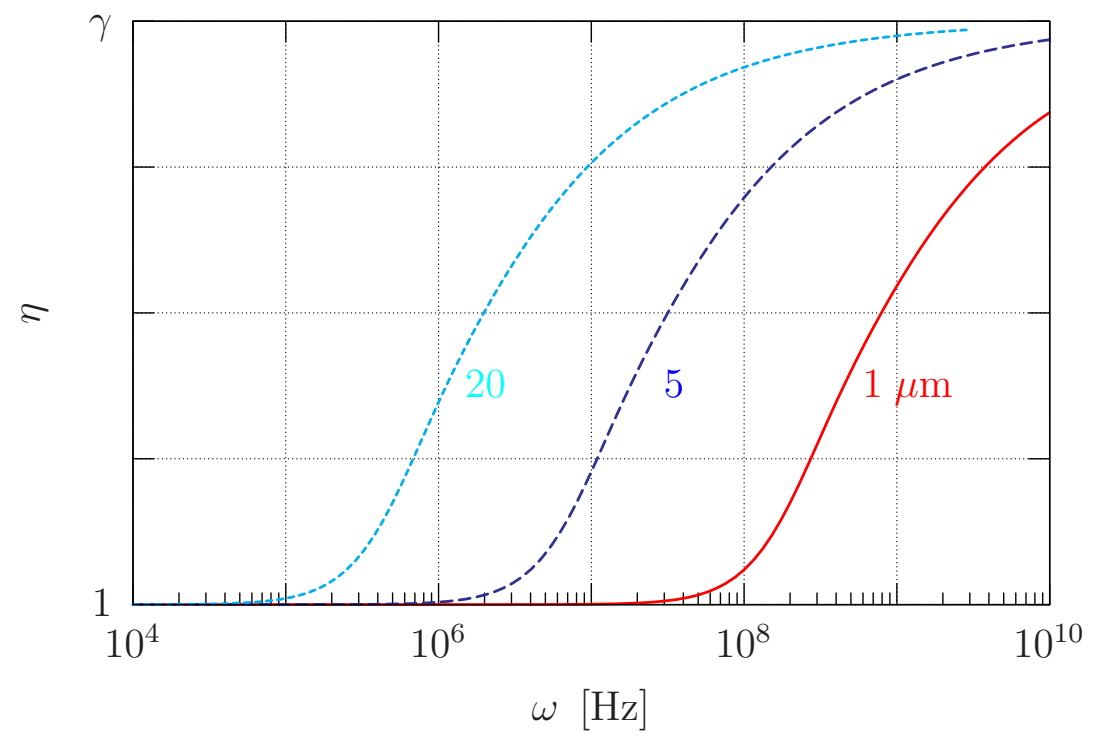

Abbildung 2.9: Gezeigt ist der Übergang des Polytropenexponenten von einem rein isothermen Prozess $(\eta=1)$ zu einem rein adiabatischen Prozess $(\eta=\gamma)$ nach (2.16) in Abhängigkeit von der Kreisfrequenz bei unterschiedlichen Ruheradien; $R_{\mathrm{n}}=1,5,20 \mu \mathrm{m}, D_{\mathrm{th}}=0.2 \cdot 10^{-4} \mathrm{~m}^{2} / \mathrm{s}$.

Eine Blase, die harmonisch dem treibenden Druckterm folgt, schwingt isotherm bei einer nicht zu hohen Anregungsfrequenz. Um die Theorie auf eine nichtlinear schwingende Blase zu übertragen, wird für diese Blase eine Frequenz angenommen, die an die Blasenwandgeschwindigkeit gekoppelt ist. Demzugrunde liegt

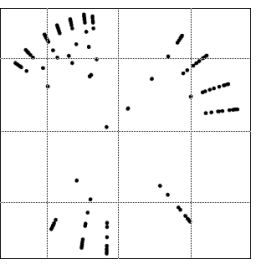


die Überlegung, dass eine schnell bewegte Blasenwand einen schnellen Kollaps der Blase und ein frühzeitiges Wiederaufschwingen bedeutet, was als eine höhere Frequenz gegenüber der treibenden Periodendauer interpretiert werden kann.

Aus der linearen Betrachtung der Blasenoszillation ergibt sich ein Ausdruck für die Frequenz der Blasendynamik:

$$
\begin{aligned}
R & =R_{\mathrm{n}}+R_{\mathrm{A}} \sin (\omega t) \\
\Rightarrow \quad \dot{R} & =\omega R_{\mathrm{A}} \cos (\omega t) \\
\Rightarrow \quad \omega & =\frac{\dot{R}}{R_{\mathrm{A}} \cos (\omega t)}
\end{aligned}
$$

\section{$R_{\mathrm{A}}=R_{\max }-R_{\mathrm{n}} \quad$ Amplitude der radialen Blasenauslenkung}

Zur Bestimmung der unteren Grenzfrequenz kann $\cos (\omega t)=1$ gesetzt werden. Da der entscheidende Einfluss der schnell bewegten Blasenwand auf den Ablauf des thermischen Prozesses in den Spitzen der Kollapsphasen auftritt, ist die Vorgeschichte der Blase, etwa ihre maximale Auslenkung, nicht ausschlaggebend. In guter Näherung wird der Betrag der Geschwindigkeit der Blasenwand auf den Ruheradius bezogen. So ergibt sich eine „momentane Frequenz", die der Blasenpulsation dynamisch angepasst ist und in Kombination mit (2.17) den Parameter $\xi$ liefert:

$$
\omega_{\mathrm{dyn}}(t)=\frac{|\dot{R}(t)|}{R_{\mathrm{n}}} \quad \Rightarrow \quad \xi=R_{\mathrm{n}} \sqrt{\frac{2|\dot{R}|}{R_{\mathrm{n}} D_{\mathrm{th}}}}
$$

Wie von Krefting et al. [19, 40] bereits in einem heuristischen Ansatz gezeigt wurde, nimmt der Polytropenexponent bei stark kollabierenden Blasen vor allem Einfluss auf den Verlauf der Nachschwinger nach dem ersten Kollaps der Blase. Diese Nachschwinger lassen sich mit einem variablen Exponenten deutlich besser an die experimentellen Beobachtungen einer kollabierenden Blase anpassen.

Abbildung 2.10 zeigt den Verlauf einer radialen Blasenpulsation und die aus der Geschwindigkeit bestimmte dynamische Frequenz.

Die Wahl des Polytropenexponenten wirkt sich auf die Phasenlage des Blasenkollaps aus und auf die Stärke der Nachschwinger. Während sich der Exponent bei einer langsamen Dynamik dem isothermen Fall annähert, strebt er in der Zeit einer schnell bewegten Blasenwand gegen den adiabatischen Grenzfall. In den Umkehrpunkten der Blasenschwingung liegt ein rein isothermer Ablauf vor. Diesen überwiegend vorliegenden isothermen Prozess während einer Pulsationsperiode zeigen auch HiLGENFELDT et al. [41] auf.

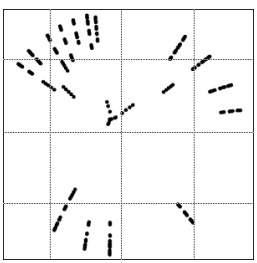




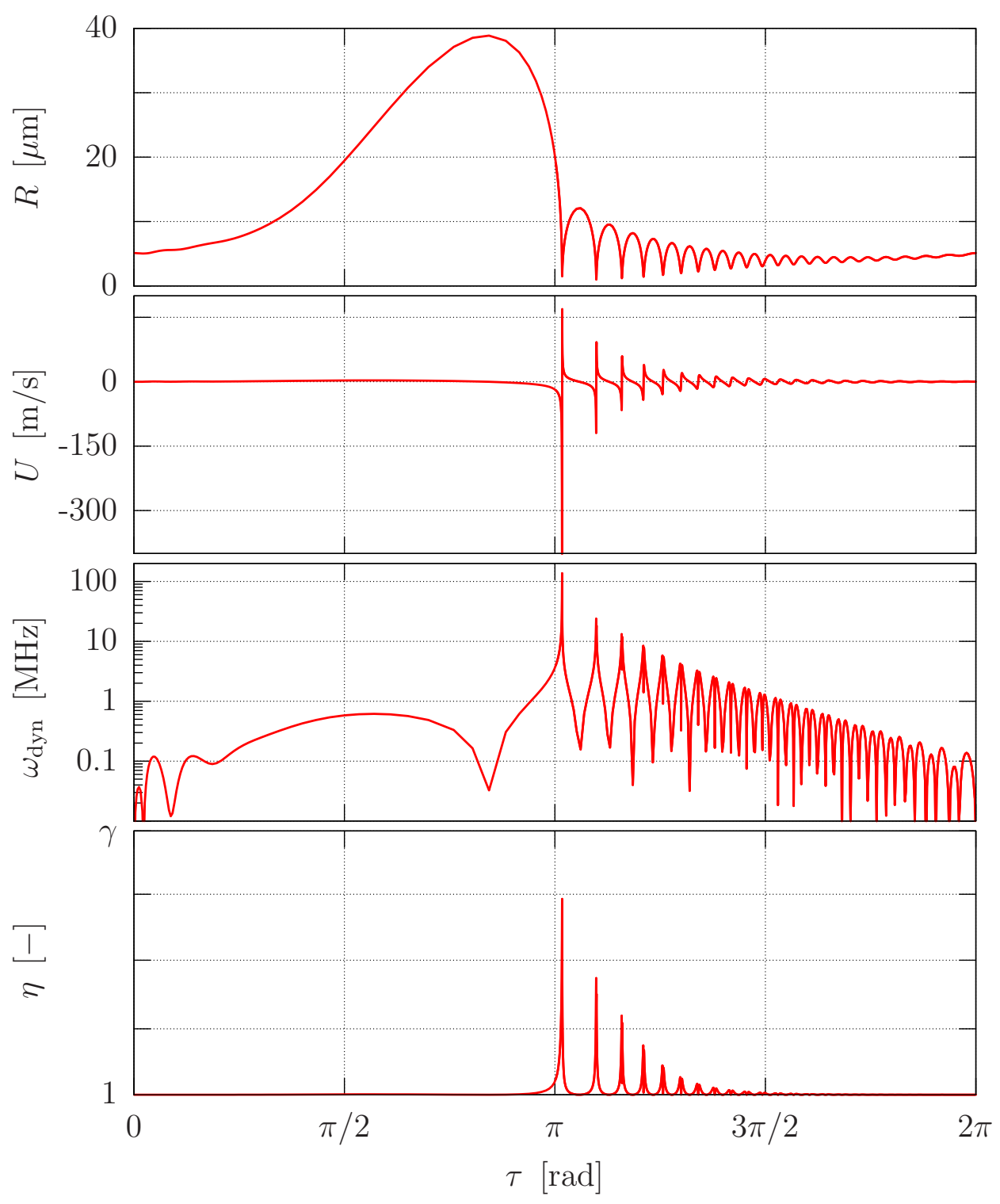

Abbildung 2.10: Darstellung von Radius und Blasenwandgeschwindigkeit der radialen Blasenpulsation nach Keller-Miksis (2.10). Weiterhin gezeigt ist der Verlauf der dynamischen Frequenz $\omega_{\text {dyn }}$ nach $(2.18)$ sowie der Verlauf des Polytropenexponenten $\eta$ nach $(2.16) ; \nu=20 \mathrm{kHz}$, $p_{\mathrm{A}}=120 \mathrm{kPa}, R_{\mathrm{n}}=5 \mu \mathrm{m}, \gamma=5 / 3$.

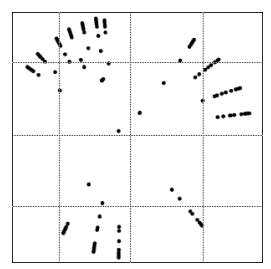




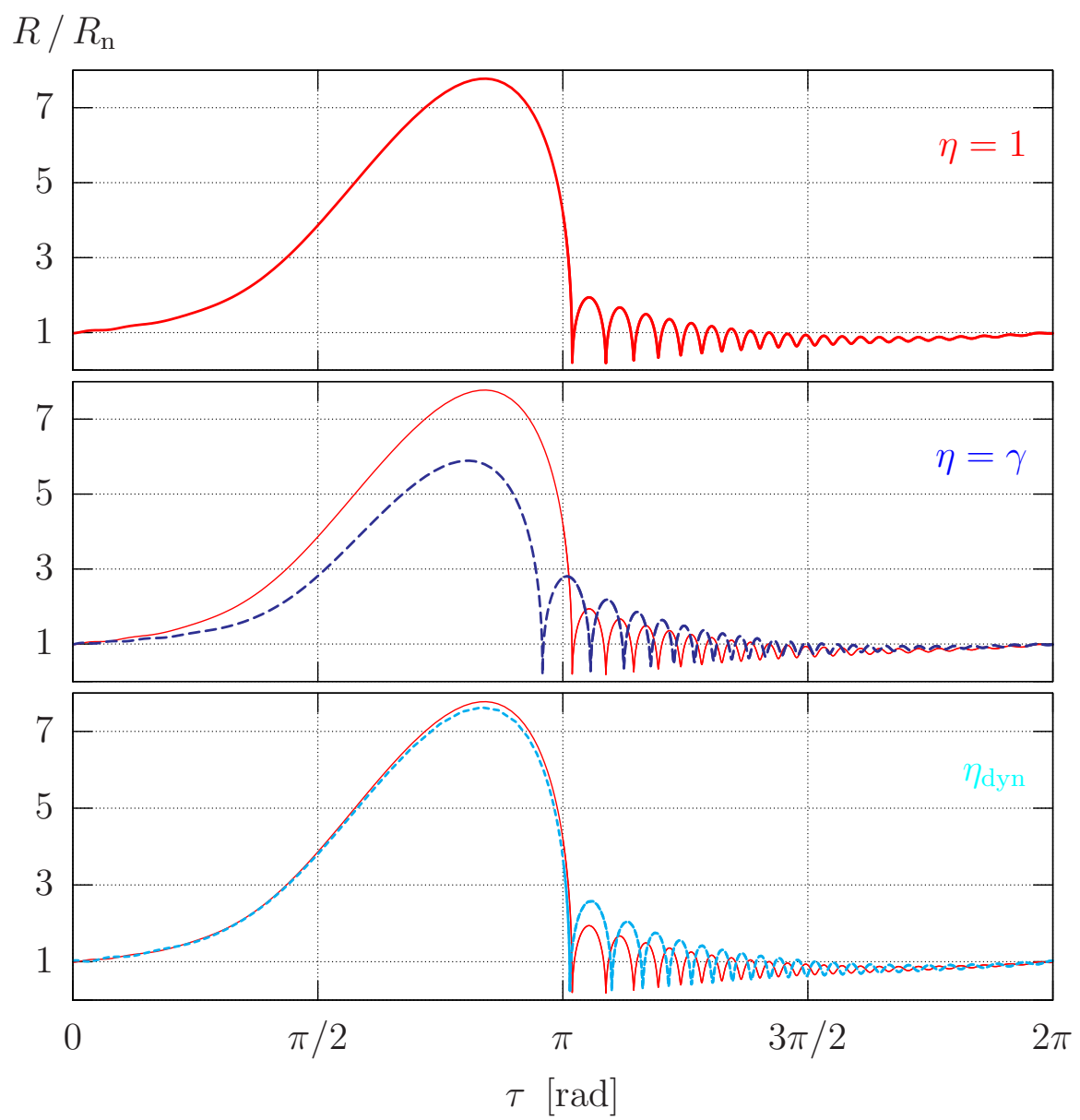

Abbildung 2.11: Gegenüberstellung der radialen Blasenoszillation nach Keller-Miksis (2.10) im rein isothermen Fall $(\eta=1)$, adiabatischem Fall $(\eta=\gamma)$ und dem dynamisch über die Frequenz nach (2.18) an die Blasenwandgeschwindigkeit gekoppelten Polytropenexponenten; $p_{\mathrm{A}}=120 \mathrm{kPa}$, $\nu=20 \mathrm{kHz}, R_{\mathrm{n}}=5 \mu \mathrm{m}, \gamma=5 / 3, D_{\mathrm{th}}=0.2 \cdot 10^{-4} \mathrm{~m}^{2} / \mathrm{s}$.

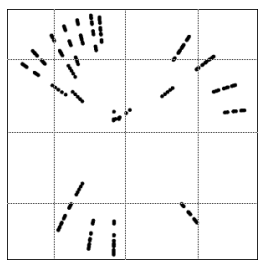


Die vorgestellten Blasenmodelle gehen von einem über das Blaseninnere konstanten Druck aus: $p_{\mathrm{i}}=p_{\text {stat }}\left(R_{\mathrm{n}} / R\right)^{3 \eta}$. Gerade für einen heftigen Kollaps kommt es jedoch zu Druckschwankungen bis hin zur Aussendung von Stoßwellen in das Blaseninnere, wie es etwa von MetTen [43] mit Methoden der Molekulardynamik untersucht wurde. Das heißt einer exakteren Modellierung des thermischen Prozesses muss eine verbesserte Beschreibung des Blaseninnendruckes vorausgehen.

Für die nachfolgenden Berechnungen in der vorliegenden Arbeit wird stets der isotherme Grenzfall eingesetzt, nachdem sich in Testsimulationen gezeigt hat, dass diese Betrachtung eine gute Näherung darstellt. Abbildung 2.11 zeigt einen Vergleich der Blasendynamik nach Keller-Miksis (2.10) in dem isothermen und adiabatischen Fall, sowie für den dynamisch angepassten Polytropenexponenten.

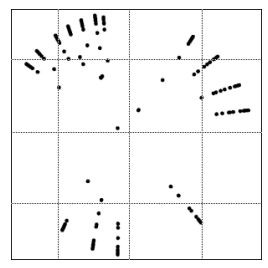




\subsection{Oberflächenmoden}

Die Stabilität der Kugelgestalt einer Blase wird durch die angeregten Oberflächenmoden bestimmt. Starke Schwingungen der Oberfläche können zur Fragmentierung der ursprünglichen Blase führen.

Zur Behandlung von Oberflächenmoden muss die Annahme einer radialen Symmetrie der Blasenoberfläche abgewandelt werden. Dies geschieht durch die Überlagerung der sphärischen Pulsation mit Auslenkungen der Oberfläche, das heißt der Blasenwandabstand zum Ursprung ist abhängig von Zeit und Winkel:

$$
\tilde{R}(t, \vartheta, \varphi)=R(t)+a(t, \vartheta, \varphi)
$$

$\tilde{R} \quad$ Blasenwandabstand zum Ursprung

$R$ sphärischer Anteil

a Anteil der Oberflächenauslenkung
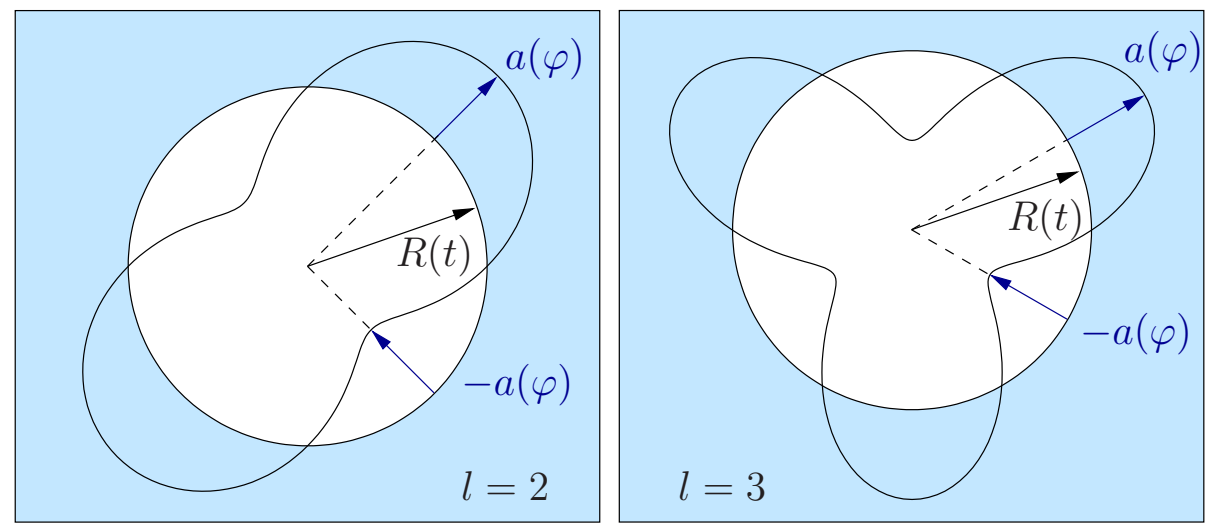

Abbildung 2.12: Zweidimensionale Darstellung zweier möglicher Oberflächenschwingungsmoden einer Blase; $l=2,3$.

Die rein radial oszillierende Blase ist eine idealisierte Annahme. In Kugelkoordinaten muss der Radius im Allgemeinen abhängig von den Winkeln $\varphi$ und $\vartheta$ sowie der Zeit $t$ beschrieben werden. Die anschwingenden Oberflächenmoden werden durch die Kugelflächenfunktionen beschrieben:

$$
\begin{aligned}
\tilde{R}(\varphi, \vartheta, t) & =R(t)+\sum_{l=1}^{\infty} \sum_{m=-l}^{l} a_{l, m}(t) Y_{l, m}(\varphi, \vartheta) \\
Y_{l, m}(\varphi, \vartheta) & =\sqrt{\frac{(2 l+1)(l-m) !}{4 \pi(l+m) !}} P_{l}^{m}(\cos \vartheta) \mathrm{e}^{\imath m \varphi} \\
P_{l}^{|m|}(\psi) & =\frac{(-1)^{m}}{2^{l} l !}\left(1-\psi^{2}\right)^{m / 2} \frac{\mathrm{d}^{l+m}}{\mathrm{~d} \psi^{l+m}}\left(\psi^{2}-1\right)^{l}
\end{aligned}
$$

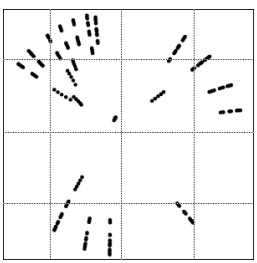




$$
\begin{aligned}
a_{l, m} & \text { Entwicklungskoeffizienten } \\
Y_{l, m}(\varphi, \vartheta) & \text { Kugelfächenfunktion } \\
P_{l}^{|m|}(\psi) & \text { zugeordnete Legendre-Polynome 1. Art } \\
l, m & \text { Modenindizes }
\end{aligned}
$$

Die Kugelflächenfunktionen bilden ein vollständiges Orthonormalsystem. Sie werden als Eigenfunktionen der möglichen Schwingungsformen einer sphärischen Blase angenommen. Eine Überlagerung aller Moden würde somit die exakte Oberflächengestalt der Blase angeben.

Eine periodische Blasenpulsation führt zu periodischen, parametrisch angeregten Schwingungen in den Entwicklungskoeffizienten, beschrieben durch lineare Differentialgleichungen zweiter Ordnung für $a_{l}$ :

$$
\ddot{a}_{l, m}+B(t) \dot{a}_{l, m}-A(t) a_{l, m}=0
$$

Der Index $m$ gibt die Entartung der Oberflächenmode $l$ an. Er wird im Folgenden nicht mehr angegeben, da er für die Stabilitätsbetrachtung keine Rolle spielt.

Die Koeffizienten $A$ und $B$ werden von PLEsset [44] für einen Flüssigkeitstropfen in einem idealen Fluid angegeben und in einer späteren Veröffentlichung von Plesset und Mitchell [45] auch für den Spezialfall einer leeren Blase. Diesen Ansatz erweiternd gibt Prosperetti [46] die Koeffizienten für ein wirbelfreies Fluid an:

$$
\begin{aligned}
& A(t)=A(R(t), \dot{R}(t), \ddot{R}(t))=(l-1)\left(\frac{\ddot{R}}{R}-\frac{l+2}{\varrho_{1} R^{3}}((l+1) \sigma+2 \mu \dot{R})\right) \\
& B(t)=B(R(t), \dot{R}(t), \ddot{R}(t))=\frac{3 \dot{R}}{R}+(l+2)(2 l+1) \frac{2 \mu}{\varrho_{1} R^{2}}
\end{aligned}
$$

Es zeigt sich, dass für Kavitationsblasen im Wesentlichen die zweite Mode angeregt wird $(l=2)$, bevor Energie in höheren Moden aufgenommen wird. Je komplizierter die Mode wird, umso mehr weicht die Oberfläche von der sie minimierenden Kugelgestalt ab, das heißt, es muss mehr Arbeit gegen die Oberflächenspannung aufgebracht werden. Aus diesem Grund sind niedrige Moden, speziell $l=2$, bevorzugt.

Die Oberflächenstabilität der Blase hängt somit direkt mit der Stabilität der angeregten Moden zusammen; bereits bei einer instabilen Mode wird die Blase instabil. Dabei werden in der Literatur im Allgemeinen zwei mögliche Instabilitäten unterschieden: 
- Bei der Parametrischen Instabilität kommt es zu einem Anwachsen der Oberflächenauslenkung mit jeder von der Blase durchlaufenen Oszillationsperiode.

Die Integration der Differentialgleichungen (2.22) für $a_{l}$ und $\dot{a}_{l}$ über eine Periode der Blasenpulsation lassen sich ersetzen durch Multiplikation mit der Monodromy-Matrix ${ }^{2}$. Ist ein Eigenwert dieser Matrix betragsmäßig größer als Eins, so kommt es mit jeder Schwingungsperiode zu einer Verstärkung, sind dagegen alle Eigenwerte kleiner Eins, stellt dies eine Dämpfung des Systems dar:

$$
\max \left(\left|\lambda_{1,2}\right|\right) \quad\left\{\begin{array}{l}
>1 \text { instabil } \\
\leq 1 \text { stabil }
\end{array}\right.
$$

\section{$\lambda_{1,2} \quad$ Eigenwerte der Monodromy-Matrix}

Die Viskosität der Flüssigkeit geht in die Berechnung ein, dagegen unberücksichtigt bleiben andere Verluste wie Schallabstrahlung, Wirbelbildung, Wärmeleitung und die Anregung höherer Moden. Um diese Verluste nicht gänzlich außer Acht zu lassen, kann statt der Stabilitätsgrenze für $\max \left(\left|\lambda_{1,2}\right|\right)=1$ ein höherer Wert etwa $10 \ldots 100$ angenommen werden, womit der stärkeren Dämpfung des System Rechnung getragen wird, wie von Krefting et al. [48] ausgeführt.

Abbildung 2.13 zeigt die Verschiebung der parametrischen Stabilitätsgrenze für unterschiedliche maximale Eigenwerte in der Parameterebene von Ruheradius und Schalldruckamplitude.

Das System weist eine Grenze bei $R_{\mathrm{n}} \approx 5 \mu \mathrm{m}$ auf, unterhalb derer Stabilität vorherrscht. Ebenso kann in weiten Bereichen für $p_{\mathrm{A}}<80 \mathrm{kPa}$ das Blasensystem als stabil angesehen werden. Um die unterschätzte Dämpfung der Oberflächenmode auszugleichen, können höhere Eigenwerte als Stabilitätsgrenze angesetzt werden, womit sich der Stabilitätsbereich zu höheren Ruheradien und Schalldruckamplituden hin ausweitet.

- Für die Beurteilung der in Abbildung 2.14 gezeigten Rayleigh-TaylorInstabilität wird der zeitliche Verlauf der Blasenoszillation über ihre Pulsationsperiode beobachtet. Eine Blase gilt als instabil, sobald mindestens einmal die Auslenkung der Oberfläche $a_{l}$ den Radius $R$ überschreitet:

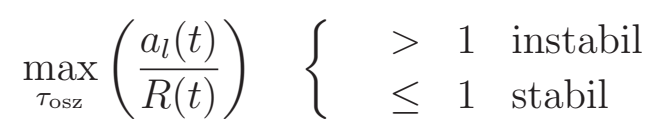

\footnotetext{
2 Dem zu Grunde liegt die Floquet-Theorie, siehe zum Beispiel Bronstein et al. [47].
}

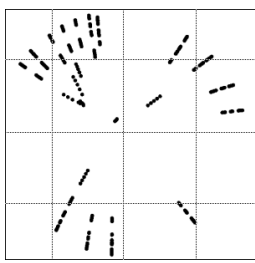




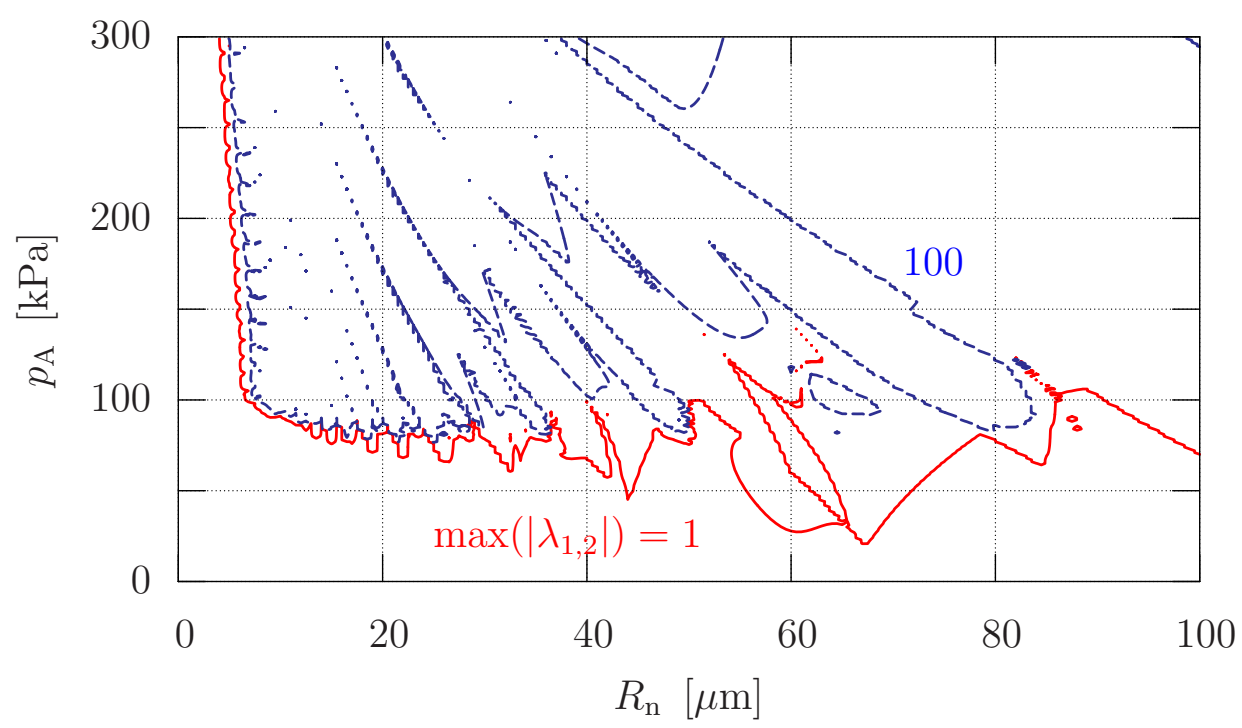

Abbildung 2.13: Betrachtung der parametrischen Stabilität des an die Keller-Miksis-Gleichung (2.10) gekoppelten Systems (2.22) in der $p_{\mathrm{A}^{-}} R_{\mathrm{n}^{-}}$ Parameterebene. Eingezeichnet sind die Übergänge zwischen stabilen und instabilen Gebieten. Der Stabilitätsbereich verkleinert sich mit zunehmender Dämpfung (höherer Eigenwert); $\max \left(\left|\lambda_{1,2}\right|\right)=1,100, \nu=20 \mathrm{kHz}$, $\eta=1$ (isotherm).

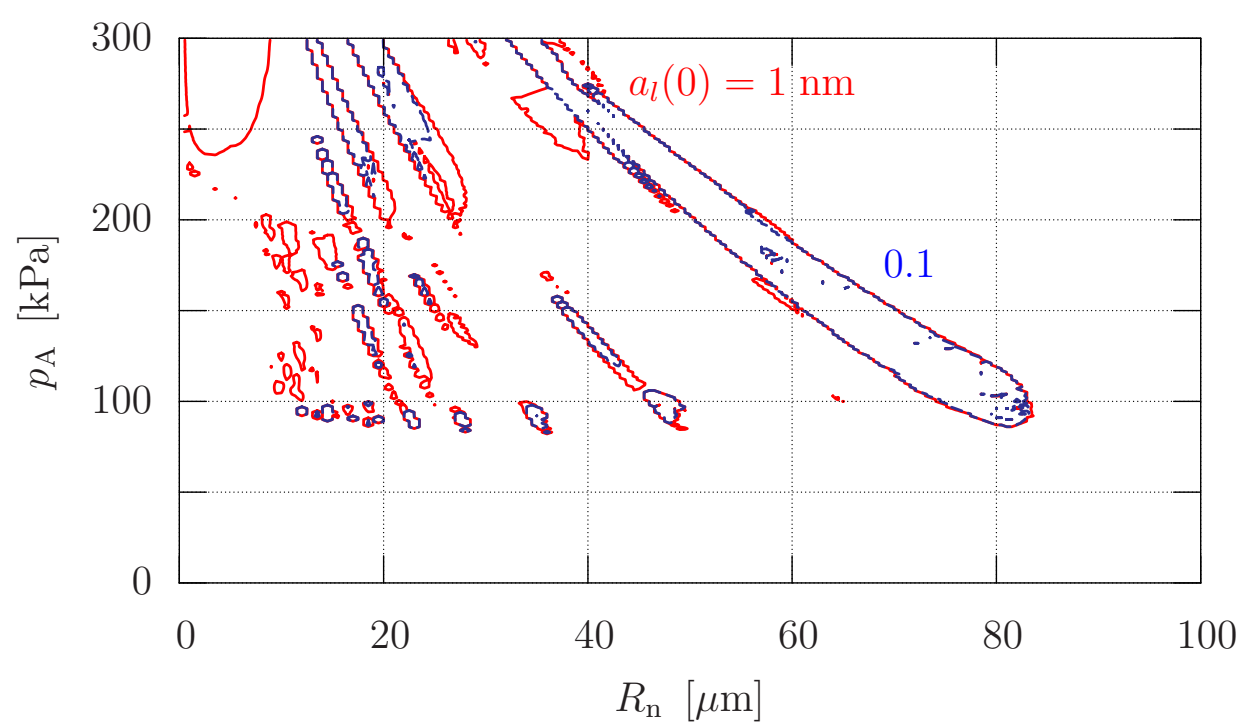

Abbildung 2.14: Betrachtung des Rayleigh-Taylor-Stabilitätskriteriums für unterschiedliche Grundauslenkungen der Oberfläche. Eingezeichnet sind die Übergänge zwischen stabilen und instabilen Gebieten. Mit zunehmender Anfangsauslenkung vergrößert sich der instabile Bereich; $a_{l}(0)=$ $0.1,1 \mathrm{~nm}, \nu=20 \mathrm{kHz}, \eta=1$ (isotherm).

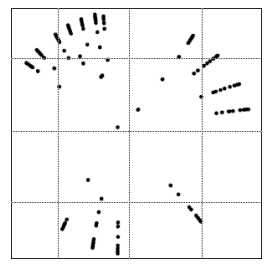


Da hier die absolute Oberflächenauslenkung eine Rolle spielt, muss $a_{l}(t=0)$ mit einem realistischen Wert initialisiert werden. Üblich sind hier einige Nanometer. Der Stabilitätsbereich bei entsprechender Initialisierung ist in Abbildung 2.14 gezeigt. Die Oberflächendynamik der $a_{l}(t)$ ist einseitig getrieben von der radiale Blasendynamik $R(t)$. Das heißt die Blasendynamik $R(t)$ verläuft unabhängig von der linearen Oberflächendynamik $a_{l}(t)$. Zudem ist die Differentialgleichung (2.22) von $a_{l}(t)$ linear, womit die gesamte Oberflächenauslenkung $a_{l}(t)$ linear mit der Anfangsauslenkung $a_{l}(t=0)$ skaliert.

Qualitativ ist das Rayleigh-Taylor stabile Regime vergleichbar mit den Bereichen der parametrischen Stabilität in Abbildung 2.13. Es zeigen sich in beiden Fällen die aus den Resonanzen der Blase hervorgehenden „Stabilitätszungen“.

Mit Annahme einer größeren Anfangsauslenkung der Oberfläche, weitet sich das nach Rayleigh-Taylor instabile Gebiet aus. Die Gegenüberstellung beider Kriterien in Abbildung 2.15 verdeutlicht, dass die Instabilitätsbereiche nach RayleighTaylor innerhalb der parametrischen Stabilitätsgrenze bleiben. In dem parametrisch stabilen Bereich wird die Oberflächenauslenkung für die in Abbildung 2.15 gezeigten Parameterwerte über eine Pulsationsperiode so stark gedämpft, dass es zu keiner den Blasenradius übersteigenden Störung kommt.

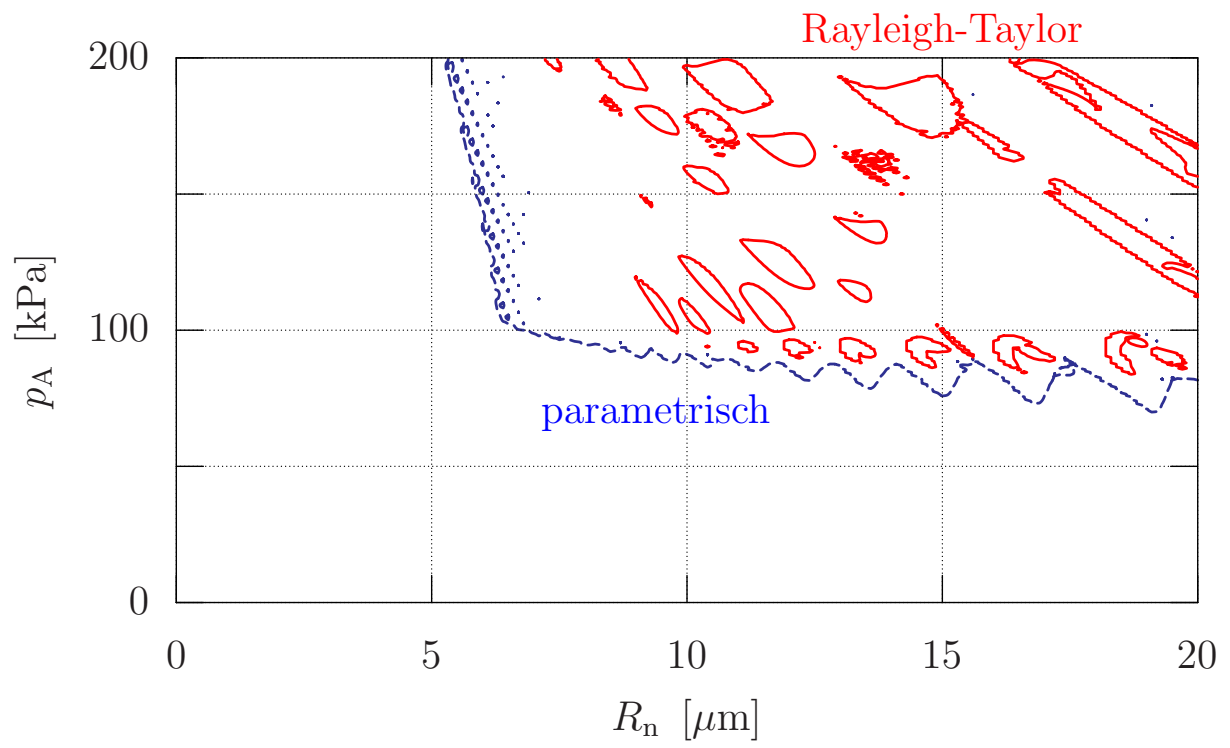

Abbildung 2.15: Ausschnittsvergrößerte Gegenüberstellung der parametrischen Stabilitätsgrenze aus Abbildung 2.13 und der Rayleigh-TaylorStabilität aus Abbildung 2.14; $\nu=20 \mathrm{kHz}, \max \left|\lambda_{1,2}\right|=1, a_{l}(0)=1 \mathrm{~nm}$.

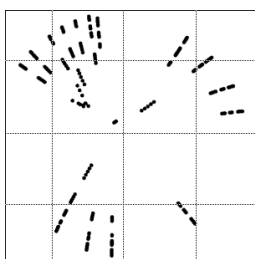




\subsection{Diffusion}

Der Gasgehalt einer Blase ist im Allgemeinen nicht konstant. Diffusionsprozesse durch die Blasenwand führen über die Zeit zu einer Änderung der Blasenruhegröße:

$$
R_{\mathrm{n}}=R_{\mathrm{n}}\left(t, R_{0}, p_{\mathrm{A}}\right)
$$

$R_{0} \quad$ Ausgangsruheradius $R_{0}=R_{\mathrm{n}}(t=0)$

In einer ruhenden Flüssigkeit wirkt der Umgebungsdruck, bestehend aus Luftdruck $p_{\text {Luft }}$ und hydrostatischem Druck $p_{\text {hyd }}$. Der Innendruck $p_{\text {i }}$ einer nicht angetriebenen Blase in dem Fluid kompensiert gerade den statischen Umgebungsdruck $p_{\text {stat }}$ und die die Blase zusammenziehende Oberflächenspannung $p_{\sigma}$. Der hydrostatische Druck $p_{\text {hyd }}$, also der Druck der Wassersäule, kann in der Regel gegenüber dem Luftdruck vernachlässigt werden, so dass $p_{\text {stat }}=p_{\text {Luft }}$ :

$$
p_{\mathrm{i}}=p_{\text {stat }}+p_{\sigma}>p_{\text {stat }}=p_{\infty}
$$

$$
\begin{aligned}
p_{\mathrm{i}} & \text { Gasdruck im Blaseninneren } \\
p_{\text {stat }} & \text { statischer Druck } \\
p_{\sigma}=2 \sigma / R & \text { Oberflächendruckterm } \\
\sigma & \text { Oberflächenspannung } \\
p_{\infty} & \text { Druck im Fluid in großer Entfernung von der Blase }
\end{aligned}
$$

Sogar im Fall einer gesättigten Lösung würde demnach aufgrund der Oberflächenspannung jede statische Blase vollständig in Lösung diffundieren. Das heißt, eine Blase kann nur eine statische Gleichgewichtslage bezüglich der Diffusion erreichen, sofern die Flüssigkeit mit Gas übersättigt ist. Es ist nun aber auch möglich, dass durch Volumenoszillationen ein dynamisches mittleres Gleichgewicht erreicht wird.

Die Verknüpfung von Konzentration und Partialdruck des entsprechenden Gases über dem Fluid gibt bei niedrigen Konzentrationen das Henry'sche Gesetz an:

$$
p=k_{\mathrm{H}} c
$$

p Partialdruck einer Gasteilchensorte

$k_{\mathrm{H}} \quad$ Henry'sche Konstante

c Konzentration der Teilchensorte in der Flüssigkeit

Bei einer oszillierenden Blase treten drei für die Diffusion wichtige Effekte auf, die von ELLER und FLYNN [49] beschrieben werden. Sie bewirken eine sogenannte gleichgerichtete Diffusion, das heißt innerhalb einer Schwingungsperiode besteht ein in die Blase gerichteter Netto-Massenfluss. 
1. Bei einer über den Ruheradius aufschwingenden Blase sinkt der Innendruck $p_{\mathrm{i}}$ unter den Druck in der umgebenden Flüssigkeit $p_{\mathrm{a}}$, was den Massenfluss in die Blase verstärkt, siehe Abbildung 2.16.

In der umgekehrten Oszillationsphase schrumpft die Blase, mit der Kompression des Gases wächst der Innendruck an, wodurch Gas in die Flüssigkeit gedrückt wird.
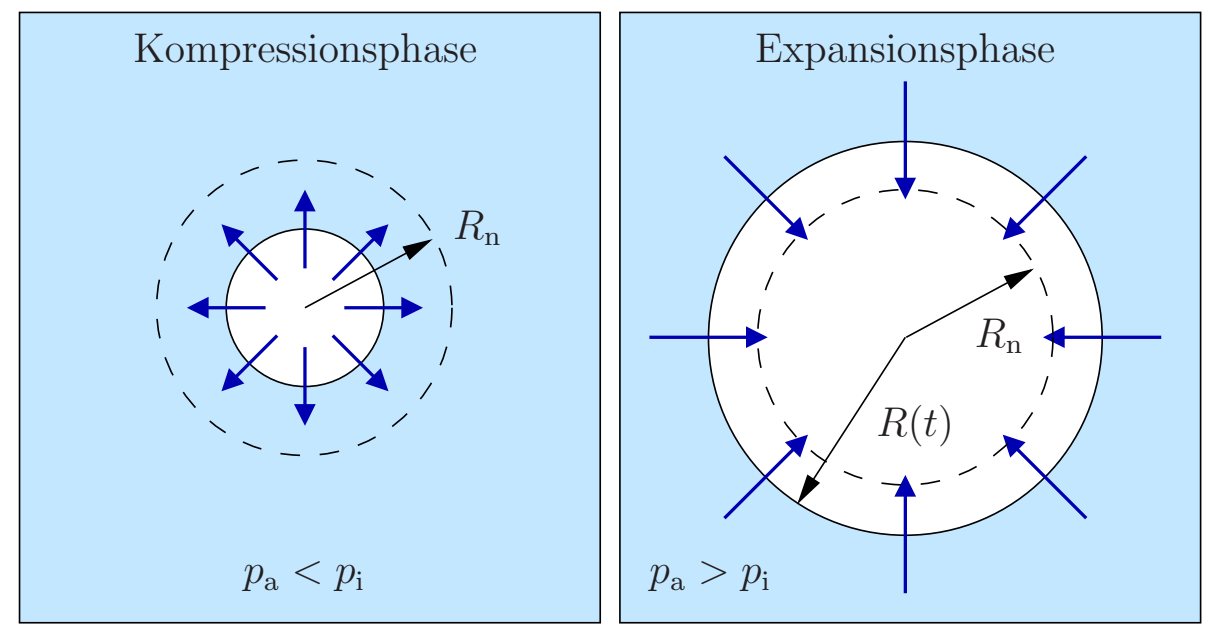

Abbildung 2.16: Abhängigkeit der Diffusion von der momentanen Blasengröße, innerhalb der Pulsationsperiode; links: In der Kompressionsphase geht Gas in Lösung; rechts: Diffusion von Gas in die Blase hinein während der Expansionsphase.

Im Fall einer stark nichtlinear schwingenden Blase befindet sich die Blase für einen längeren Zeitraum im Bereich oberhalb ihres Ruheradius als unterhalb, so wird im Mittel über eine Periode die Diffusion in die Blase begünstigt.

2. Mit dem Aufschwingen der Blase vergrößert sich ihre Oberfläche $A \sim R^{2}(t)$, und umgekehrt sinkt die Oberfläche unter die Fläche in der Ruhelage $A_{n}=$ $4 \pi R_{\mathrm{n}}^{2}$ während der Kollapsphasen der Blasenoszillation.

Da der Massenfluss proportional zur Oberfläche ist, begünstigt das wiederum die Diffusion nach innen:

$$
\frac{\mathrm{d} m}{\mathrm{~d} t} \sim 4 \pi R^{2}(t)
$$

$m$ Masse des Gases in der Blase

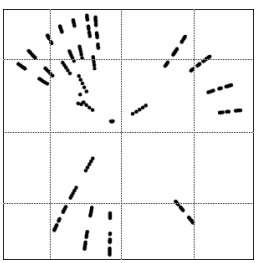


3. Im Fluid stellt sich über die Zeit eine gleichmäßige Konzentrationsverteilung ein. Durch Diffusionsprozesse während der Blasenoszillation kommt es an der Gas-Fluid-Grenzschicht zu einem Gradienten der Konzentration innerhalb des Flüssigkeitsvolumens, mit dem der Gasaustausch stattfindet.

Der Schaleneffekt drückt aus, dass sich ein Element konstanten Volumens an der Blasenwand für eine große Blase über einem größeren Oberflächenelement verteilt, sich dagegen bei einer kleinen Blase stärker in radialer Richtung erstreckt (vergleiche Abbildung 2.17).

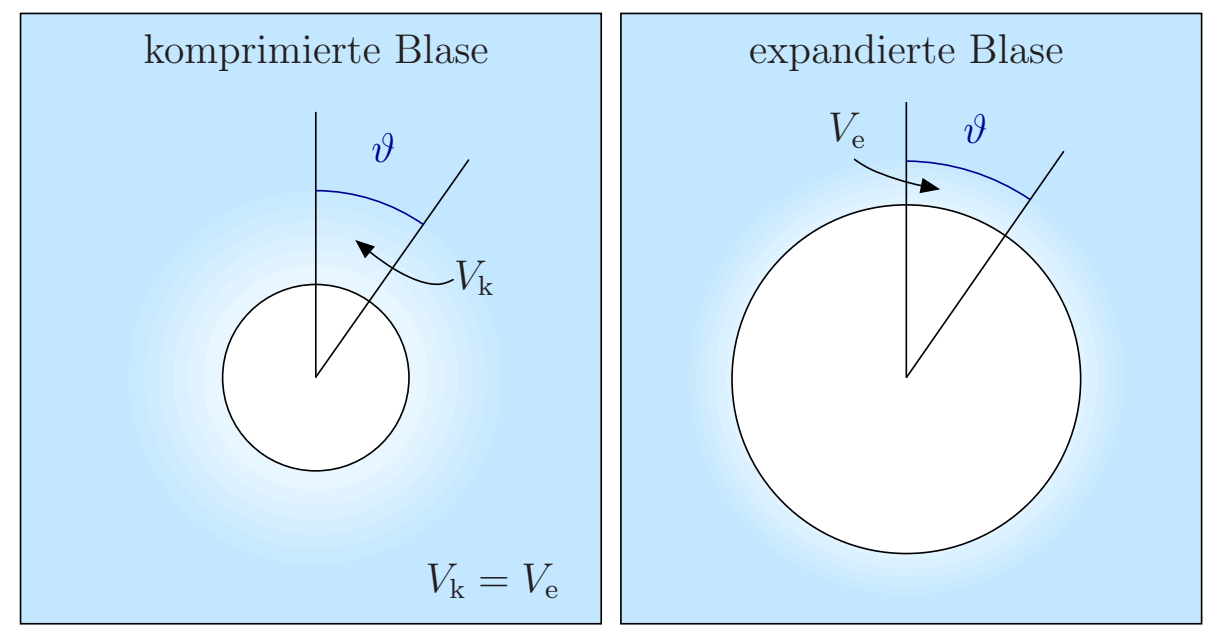

Abbildung 2.17: Aus der Blase in Lösung gehendes Gas verteilt sich auf kurzen Zeitskalen zunächst in der Nahumgebung der Blase auf ein fixes Volumen. Abhängig von der Gasmenge dehnt sich das Volumen in radialer Richtung aus, wodurch der Konzentrationsgradient über diesen Schalen variiert wird; links: schwacher Gradient an der Grenzschicht einer kleinen Blase; rechts: starker Konzentrationsgradient an einer großen Blase.

Die Stauchung des Volumenelements ist gleichbedeutend mit einem steileren Konzentrationsgradienten, womit sich bei aufgezogener Blase ein stärkerer „Konzentrationsdruck“ in Richtung der Blase einstellt.

Der durch das erste Fick'sche Gesetz beschriebene Massenfluss setzt sich aus den drei gerade beschriebenen Effekten zusammen:

$$
\frac{\mathrm{d} m}{\mathrm{~d} t}=\left.4 \pi R^{2}(t) D_{\mathrm{R}} \frac{\partial c}{\partial r}\right|_{r=R}
$$

$D_{\mathrm{R}} \quad$ Diffusionskonstante

$R$ Radius der Blase

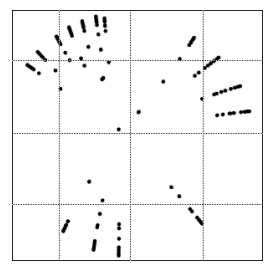


Innerhalb der Blase $(r<R)$ wird eine über den Raum gleichmäßige Konzentration angenommen. Die Konzentration im angrenzenden Fluid ergibt sich als Lösung der radialsymmetrischen Diffusionsgleichung (zweites Fick'sches Gesetz):

$$
\frac{\mathrm{d} c}{\mathrm{~d} t}=\frac{\partial c}{\partial t}+\vec{v}_{r} \cdot \nabla c=D \nabla^{2} c, \quad \vec{v}_{r}=\frac{R^{2} U}{r^{2}} \frac{\vec{r}}{r}
$$

Für die Lösung der Diffusionsgleichung (2.27) beziehen ELLER und FLYNN [49] nichtlineare radiale Blasenpulsationen ein. Die Schwierigkeit der Behandlung einer bewegten Grenzfläche lösen sie durch die getrennte Betrachtung der Blasenoszillation und der Diffusion.

Die Entkopplung der Oszillation und der Diffusion ist gerechtfertigt, so lange die Blasenpulsation auf deutlich schnelleren Zeitskalen abläuft als der langsamere Diffusionsprozess. Ausgedrückt wird das Verhältnis der Zeitskalen durch die Péclet-Zahl:

Diffusionszeitskala: $\quad \tau_{\text {diff }}=R^{2} / D_{\mathrm{R}}$ dynamische Zeitskala: $\tau_{\text {osz }}=R /|\dot{R}|$

$$
P e=\frac{\tau_{\text {diff }}}{\tau_{\mathrm{osz}}}=\frac{R|\dot{R}|}{D_{\mathrm{R}}}
$$

Ausgehend von der Diffusionsgleichung (2.27) und dem Massenfluss (2.26) ergibt sich die Änderung der Teilchenzahl in der Blase unter Annahme einer sphärischen Oberfläche und unter Vernachlässigung der schnellen Blasenpulsation gegenüber der Diffusion (große Péclet-Zahl):

$$
\begin{aligned}
\frac{\mathrm{d} n}{\mathrm{~d} t}= & 4 \pi R_{\mathrm{n}} D_{\mathrm{R}}\left(\left\langle R / R_{\mathrm{n}}\right\rangle_{\tau_{\text {osz }}}+R_{\mathrm{n}} \sqrt{\frac{\left\langle\left(R / R_{\mathrm{n}}\right)^{4}\right\rangle_{\tau_{\text {osz }}}}{\pi D_{\mathrm{R}} t}}\right) \\
& \cdot c_{\text {sat }}\left(\frac{c_{\infty}}{c_{\text {sat }}}-\frac{\left\langle\left(p_{\mathrm{i}} / p_{\text {stat }}\right)\left(R / R_{\mathrm{n}}\right)^{4}\right\rangle_{\tau_{\text {osz }}}}{\left\langle\left(R / R_{\mathrm{n}}\right)^{4}\right\rangle_{\tau_{\text {osz }}}}\right)
\end{aligned}
$$

$n$ molare Anzahl von Gasmolekülen in der Blase

$c_{\infty}$ molare Gaskonzentration im Fluid in großer Entfernung von der Blase

$c_{\text {sat }}$ molekülabhängige Sättigungskonzentration des Fluids

Die Sättigungskonzentration für eine bestimmte Molekülspezies ergibt sich aus dem Henry'schen Gesetz (2.25):

$$
c_{\mathrm{sat}}=p_{\mathrm{stat}} / k_{\mathrm{H}}
$$

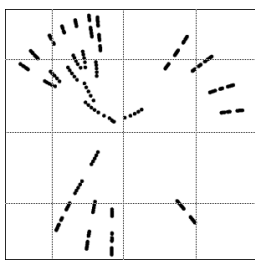


Die zeitliche Mittelung ist definiert als:

$$
\langle f\rangle_{\tau_{\mathrm{osz}}} \equiv \frac{1}{\tau_{\mathrm{osz}}} \int_{0}^{\tau_{\mathrm{osz}}} f \mathrm{~d} t
$$

$\tau_{\text {osz }}=1 / \nu \quad$ Schallfeldperiode

Das Verhältnis der Partialdrücke entspricht den Konzentrationsverhältnissen; der Normaldruck in einer ruhenden Blase setzt sich zusammen aus statischem Druck und dem Oberflächenterm:

$$
\frac{p_{\mathrm{n}}}{p_{\text {stat }}}=\frac{c_{\mathrm{g}}}{c_{\mathrm{sat}}} ; \quad p_{\mathrm{n}}=p_{\text {stat }}+\frac{2 \sigma}{R_{\mathrm{n}}}
$$

$p_{\mathrm{n}} \quad$ Normaldruck in der Blase

$c_{\mathrm{g}}$ molare Gaskonzentration im Fluid direkt an der Blasenwand

Der Blaseninnendruck ist aus (2.8) bekannt:

$$
p_{\mathrm{i}}=p_{\mathrm{n}}\left(\frac{R_{\mathrm{n}}}{R}\right)^{3 \eta} \Rightarrow \frac{p_{\mathrm{i}}}{p_{\text {stat }}}=\frac{p_{\mathrm{n}}}{p_{\text {stat }}}\left(\frac{R_{\mathrm{n}}}{R}\right)^{3 \eta}=\frac{c_{\mathrm{g}}}{c_{\mathrm{sat}}}\left(\frac{R_{\mathrm{n}}}{R}\right)^{3 \eta}
$$

In der Betrachtung des isothermen Falles $(\eta=1)$ erhält ELLER [50] mit diesem Ausdruck für das Druckverhältnis die Änderung der Teilchenzahl:

$$
\frac{\mathrm{d} n}{\mathrm{~d} t}=4 \pi R_{\mathrm{n}} D_{\mathrm{R}}\left(\left\langle\frac{R}{R_{\mathrm{n}}}\right\rangle_{\tau_{\mathrm{osz}}}+R_{\mathrm{n}} \sqrt{\frac{\left\langle\left(R / R_{\mathrm{n}}\right)^{4}\right\rangle_{\tau_{\mathrm{osz}}}}{\pi D_{\mathrm{R}} t}}\right) c_{\mathrm{g}}\left(\frac{c_{\infty}}{c_{\mathrm{g}}}-\frac{\left\langle R / R_{\mathrm{n}}\right\rangle_{\tau_{\mathrm{osz}}}}{\left\langle\left(R / R_{\mathrm{n}}\right)^{4}\right\rangle_{\tau_{\mathrm{osz}}}}\right)
$$

Um die Wachstumsrate anzugeben, nimmt CRUm [51, 52] die ideale Gasgleichung an, die sich mit (2.29) umformt:

$$
p_{\mathrm{n}} V_{\mathrm{n}}=n R_{\mathrm{g}} \Theta \quad \Leftrightarrow \quad p_{\text {stat }}+\frac{2 \sigma}{R_{\mathrm{n}}}=\frac{3 n R_{\mathrm{g}} \Theta}{4 \pi R_{\mathrm{n}}^{3}}
$$

$R_{\mathrm{g}} \quad$ universelle Gaskonstante

$\Theta$ absolute Temperatur

Für die zeitliche Änderung der Molmasse $n$ folgt aus (2.32):

$$
\frac{\mathrm{d} n}{\mathrm{~d} t}=\frac{4 \pi}{R_{\mathrm{g}} \Theta}\left(p_{\text {stat }} R_{\mathrm{n}}^{2}+\frac{4 \sigma}{3} R_{\mathrm{n}}\right) \frac{\mathrm{d} R_{\mathrm{n}}}{\mathrm{d} t}
$$

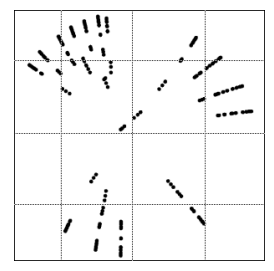


Kombiniert mit (2.31) leitet sich die Wachstumsrate für den Ruheradius ab:

$$
\begin{aligned}
\frac{\mathrm{d} R_{\mathrm{n}}}{\mathrm{d} t}= & \frac{D_{\mathrm{R}} R_{\mathrm{g}} \Theta c_{\mathrm{g}}}{p_{\mathrm{stat}} R_{\mathrm{n}}+4 \sigma / 3}\left(\left\langle\frac{R}{R_{\mathrm{n}}}\right\rangle_{\tau_{\mathrm{osz}}}+R_{\mathrm{n}} \sqrt{\frac{\left\langle\left(R / R_{\mathrm{n}}\right)^{4}\right\rangle_{\tau_{\mathrm{osz}}}}{\pi D_{\mathrm{R}} t}}\right) \\
& \cdot\left(\frac{c_{\infty}}{c_{\mathrm{g}}}-\frac{\left\langle R / R_{\mathrm{n}}\right\rangle_{\tau_{\text {osz }}}}{\left\langle\left(R / R_{\mathrm{n}}\right)^{4}\right\rangle_{\tau_{\text {osz }}}}\right)
\end{aligned}
$$

In späteren Arbeiten vernachlässigt ElLER $[50,53]$ den Term $\sim R_{\mathrm{n}} / \sqrt{t}$. Der gleiche Ansatz wird auch von CRum [51, 52] angegeben, der dabei zusätzlich den Polytropenexponenten aus (2.16) einsetzt und gute Übereinstimmungen zu Messungen an einer Kavitationsblase bei variierten Oberflächenspannungen erhält:

$$
\begin{gathered}
\frac{\mathrm{d} R_{\mathrm{n}}}{\mathrm{d} t}=\frac{D_{\mathrm{R}} R_{\mathrm{g}} \Theta c_{\mathrm{g}}}{p_{\text {stat }} R_{\mathrm{n}}+4 \sigma / 3}\left\langle\frac{R}{R_{\mathrm{n}}}\right\rangle_{\tau_{\text {osz }}}\left(\frac{c_{\infty}}{c_{\mathrm{g}}}-\frac{\left\langle\left(R / R_{\mathrm{n}}\right)^{4-3 \eta}\right\rangle_{\tau_{\text {osz }}}}{\left\langle\left(R / R_{\mathrm{n}}\right)^{4}\right\rangle_{\tau_{\text {osz }}}}\right) \\
c_{\mathrm{g}}=\left(1+\frac{2 \sigma}{R_{\mathrm{n}} p_{\text {stat }}}\right) c_{\mathrm{sat}}
\end{gathered}
$$

Die in Abbildung 2.18 gezeigten Diffusionsraten nach (2.35) verdeutlichen die Abhängigkeit der Diffusion von der Schalldruckamplitude, der Gaskonzentration in der Flüssigkeit sowie der Art der Gasmoleküle. Die Änderung der Diffusionrate mit der Schallfrequenz ist in Abbildung 2.19 aufgetragen. In allen Abhängigkeiten stellt sich ein instabiles Diffusionsgleichgewicht bei einem Ruheradius von etwa $2 \mu \mathrm{m}$ ein, das heißt Kavitationsblasen unterhalb dieses Radius lösen sich trotz gerichteter Diffusion weiter auf, dagegen wachsen Blasen oberhalb dieser Schwelle an.

Die in den jeweiligen Rechnungen eingesetzten Diffusionskonstanten und Sättigungskonzentrationen beruhen auf der von TöGEL und LoHSE [54] zusammengetragenen Aufstellung, die in Tabelle 2.1 wiedergegeben ist.

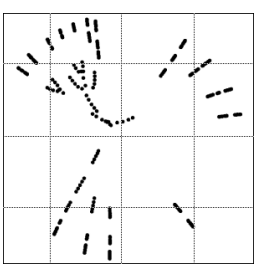




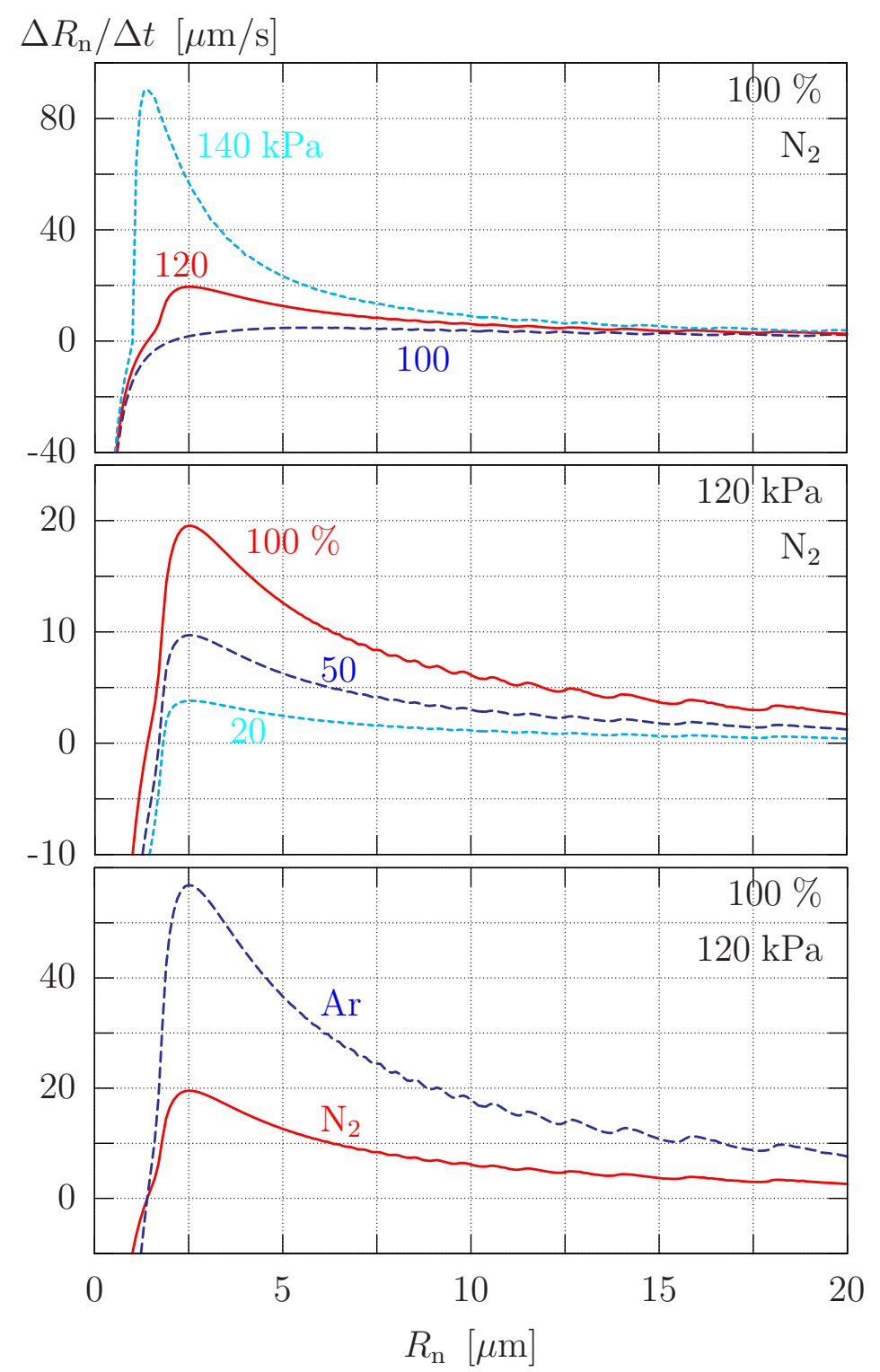

Abbildung 2.18: Darstellung der Diffusionsrate (2.35) gegen den Ruheradius. Die beiden oberen Grafiken zeigen Stickstoffmoleküle bei variierter Schalldruckamplitude bzw. Gaskonzentration. Die untere Auftragung vergleicht Argon- und Stickstoffmoleküle; $\nu=20 \mathrm{kHz}, \eta=1$ (isotherm), $p_{\mathrm{A}}=100,120,140 \mathrm{kPa}, c_{\infty} / c_{\mathrm{sat}}=20,50,100 \%$.

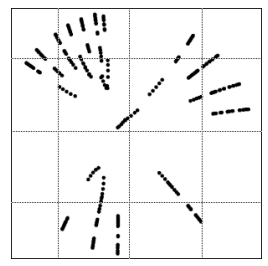




\begin{tabular}{lcc} 
Spezies & $D_{\mathrm{R}}\left[10^{-9} \mathrm{~m}^{2} / \mathrm{s}\right]$ & $c_{\mathrm{sat}}\left[\mathrm{mol} / \mathrm{m}^{3}\right]$ \\
\hline Wasserstoff $\mathrm{H}_{2}$ & 5.1 & 0.78 \\
Sauerstoff $\mathrm{O}_{2}$ & 2.4 & 1.3 \\
Stickstoff $\mathrm{N}_{2}$ & 2 & 0.6 \\
Argon Ar & 2.5 & 1.4 \\
Xenon Xe & 1.47 & 4.3
\end{tabular}

Tabelle 2.1: Zusammenstellung der Diffusionskonstanten und Sättigungskonzentrationen in Wasser für relevante Spezies in der Blase bei einem Partialdruck von $100 \mathrm{kPa}$ und einer Temperatur von $25^{\circ} \mathrm{C}$ (entnommen aus TöGEL und LOHSE [54]).

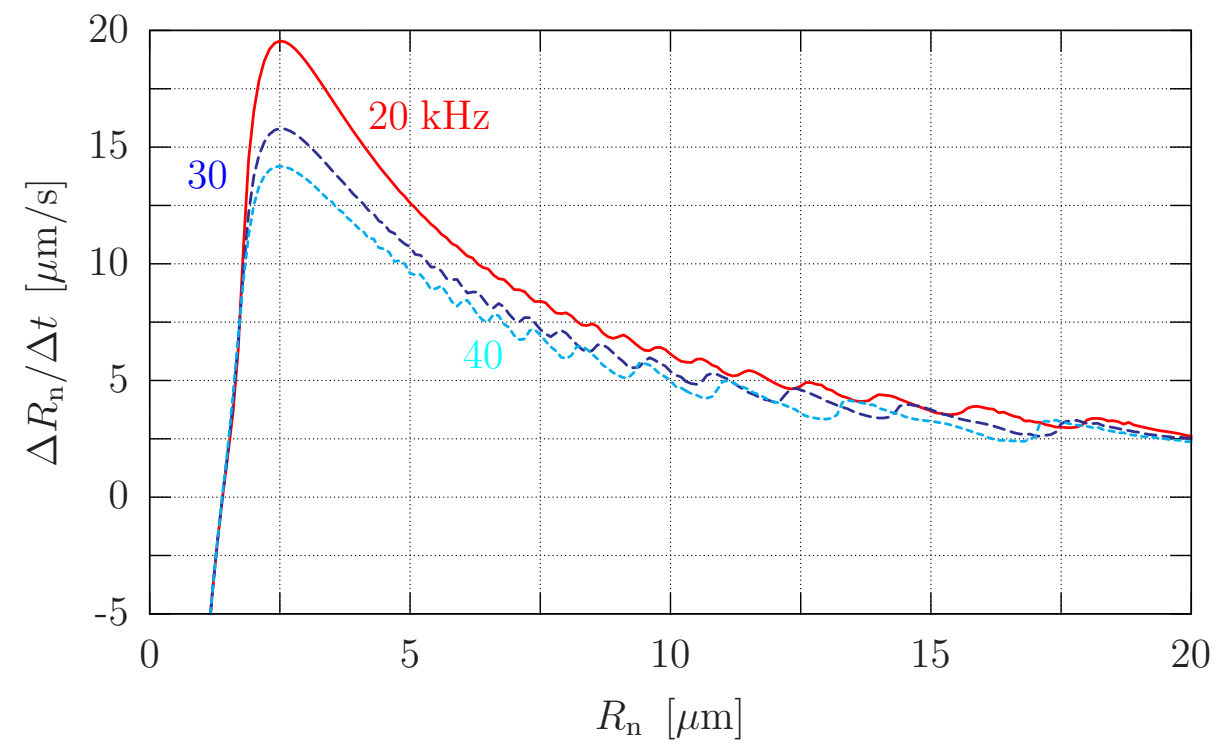

Abbildung 2.19: Diffusionsrate (2.35) von Stickstoffmolekülen bei unterschiedlichen die Blasendynamik treibenden Frequenzen; $p_{\mathrm{A}}=120 \mathrm{kPa}$, $c_{\infty} / c_{\mathrm{sat}}=100 \%, \nu=20,30,40 \mathrm{kHz}$.

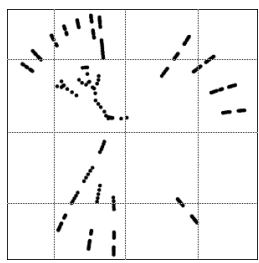


Die Lage eines Diffusionsgleichgewichts ergibt sich durch die Forderung $\mathrm{d} n / \mathrm{d} t \stackrel{!}{=} 0$ bzw. $\mathrm{d} R_{\mathrm{n}} / \mathrm{d} t \stackrel{!}{=} 0$. Nach (2.31) bzw. (2.34) bedeutet dies:

$$
\begin{gathered}
\frac{c_{\infty}}{c_{\mathrm{g}}}-\frac{\left\langle R / R_{\mathrm{n}}\right\rangle_{\tau_{\mathrm{osz}}}}{\left\langle\left(R / R_{\mathrm{n}}\right)^{4}\right\rangle_{\tau_{\mathrm{osz}}}} \stackrel{!}{=} 0 \quad \text { mit } \quad c_{\mathrm{g}}=\frac{p_{\mathrm{n}}}{p_{\text {stat }}} c_{\mathrm{sat}}=\left(1+\frac{2 \sigma}{R_{\mathrm{n}} p_{\mathrm{stat}}}\right) c_{\mathrm{sat}} \\
\Rightarrow \quad \frac{c_{\infty}}{c_{\mathrm{sat}}}-\left(1+\frac{2 \sigma}{R_{\mathrm{n}} p_{\text {stat }}}\right) \frac{\left\langle R / R_{\mathrm{n}}\right\rangle_{\tau_{\mathrm{osz}}}}{\left\langle\left(R / R_{\mathrm{n}}\right)^{4}\right\rangle_{\tau_{\mathrm{osz}}}}=0
\end{gathered}
$$

Die Auftragungen in Abbildung 2.20 zeigen die Abgrenzung in der Parameterebene von Ruheradius und Schalldruckamplitude, unterhalb derer sich Blasen trotz gleichgerichteter Diffusion aufösen. Je nach Sättigung des Fluids ist ein Mindestwert der Druckamplitude erforderlich, damit die Blasen die Gleichgewichtslage überschreiten und anwachsen können. Dieser Wert steigt mit abnehmendem Sättigungsgrad, also steigender Entgasung des Wassers.

Oberhalb der Gleichgewichtslage wächst bei konstanter Schalldruckamplitude eine zunächst kleine Kavitationsblase schnell an, wobei die Diffusionsrate mit zunehmenden Ruheradius abnimmt. Unterhalb der Gleichgewichtslage löst sich eine große Blasen zunächst langsam auf, mit abnehmenden Radius beschleunigt sich dieser Vorgang. Durch dieses Verhalten bestehen bei der Sättigung von $100 \%$ in Abbildung 2.20 nur instabile Gleichgewichtslagen. In den Bereichen einer positiven Steigung in dem Verlauf des Gleichgewichtes stellt sich allerdings eine stabile Lage ein. Beispielsweise ist bei einer Sättigung von $1 \%$ die Erhaltung einer diffusionsstabilen Blase über große Parameterbereiche möglich. ${ }^{3}$

3 Der Gasgehalt ist somit entscheidend beim Einsatz von „Blasenfallen“, etwa bei Untersuchungen der Einzelblasen-Sonolumineszenz. 


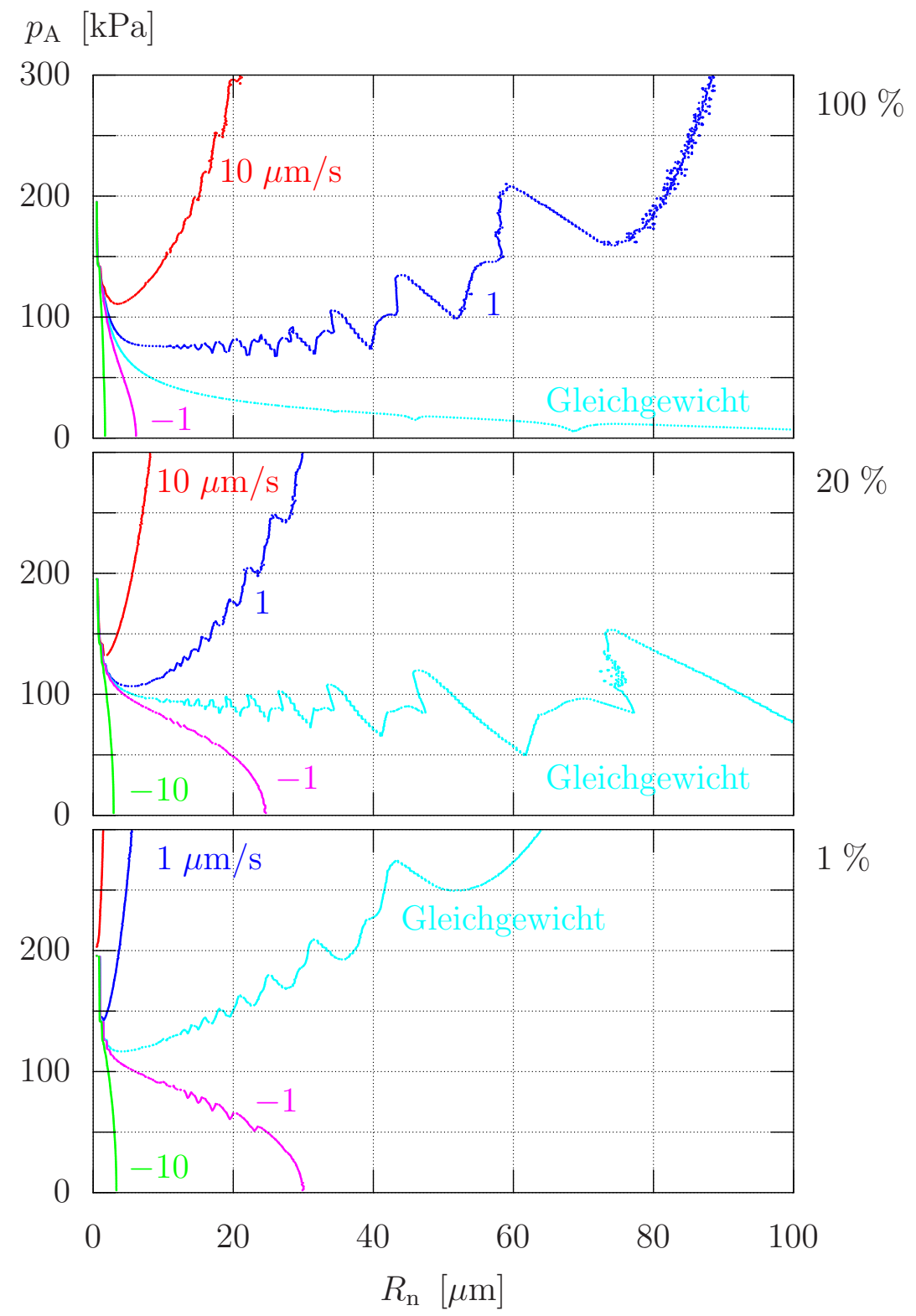

Abbildung 2.20: Anwachsrate von Kavitationsblasen in Wasser durch gleichgerichtete Diffusion von Stickstoffmolekülen in der Parameterebene aus Ruheradius und Schalldruckamplitude. Die Höhenlinien zeigen die Diffusionsraten an, Diffusionsgleichgewicht besteht für $0 \mu \mathrm{m} / \mathrm{s}$; $\Delta R_{\mathrm{n}} / \Delta t=-10,-1,0,1,10 \mu \mathrm{m} / \mathrm{s}, \nu=20 \mathrm{kHz}, \eta=1$ (isotherm), $c_{\infty} / c_{\text {sat }}=100,20,1 \%$.

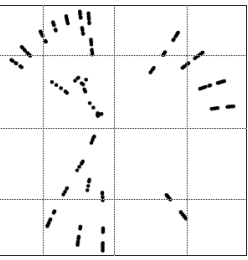




\section{Kapitel 3}

\section{Struktur des Schallfeldes}

Um die Blasendynamik an den verschiedenen Positionen in einem Resonator angeben zu können, muss die Schalldruckverteilung bestimmt sein. Deren räumliche und zeitliche Entwicklung wird unter der Annahme idealer linearer Akustik von der Wellengleichung beschrieben:

$$
\frac{1}{C_{1}^{2}} \frac{\partial^{2} \tilde{p}}{\partial t^{2}}-\Delta \tilde{p}=0
$$

$\tilde{p}=\tilde{p}(x, t) \quad$ Schallwechseldruck

$C_{1} \quad$ Schallgeschwindigkeit in der Flüssigkeit

Mit Vorgabe der Randbedingung lassen sich für einfache Geometrien analytische Lösungen der Wellengleichung angeben (Abschnitt 3.1).

Um die Schalldruckverteilung bei komplizierteren Geometrien berechnen zu können, wird die Wellengleichung mit der Methode der Finiten Elemente gelöst. Ebenso kann das Resonanzspektrum bestimmt werden, dessen Spitzen sich mit den Eigenfrequenzen bzw. -moden des betrachteten Resonators decken (Abschnitt $3.2)$.

Die Einbeziehung einer ortsabhängigen Blasenverteilung in der Küvette und der in Kapitel 2 vorgestellten nichtlinearen Blasendynamik führt in Abschnitt 3.3 auf eine erweiterte Wellengleichung nach van Wijngaarden. Sie wird in dieser Arbeit zur Beschreibung der Wellenausbreitung im kavitierenden Medium verwendet. Die volle nichtlineare Version unter Verwendung der Keller-Miksis-Gleichung kann aus numerischen Gründen jedoch nur im Eindimensionalen beispielhaft gelöst werden (Abschnitt 3.3.2).

Um die Schallfeldverteilung im Dreidimensionalen lösen zu können, bedarf es aus numerischen Gründen der Vereinfachung der vollen van Wijngaarden-

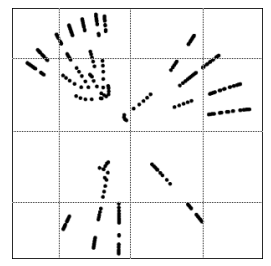


Wellengleichung. Dazu wird in Abschnitt 3.3.3 die linearisierte Keller-MiksisGleichung eingesetzt. Anschließend wird eine zeitharmonische Lösung der Wellengleichung vorausgesetzt also eine „eingeschwungene“ Lösung:

$$
\tilde{p}(\vec{x}, t)=p_{\mathrm{A}}(\vec{x}) \sin (\omega t-\varphi(\vec{x}))
$$

$p_{\mathrm{A}} \quad$ Schallfeldamplitude

$\varphi$ Phasenlage des Schallfeldes

$\omega=2 \pi \nu \quad$ Kreisfrequenz des Schallfeldes

Mit dieser Annahme wird die Ausbildung zeitharmonischer Modenstrukturen beschrieben, die von der Frequenz, der Resonatorgeometrie sowie dem Medium und gegebenenfalls von der Blasenverteilung abhängen. Wird dieser zeitharmonische Lösungsansatz (3.2) in die Wellengleichung (3.1) eingesetzt, so entkoppeln örtliche und zeitliche Abhängigkeit, was auf die Helmholtz-Gleichung führt:

$$
\frac{\omega^{2}}{C_{1}^{2}} p_{\mathrm{A}}+\Delta p_{\mathrm{A}}=0
$$

\section{$k=\omega / C_{1} \quad$ Wellenzahl}

Die numerische Analyse eines Resonators führt ohne Einbeziehung einer Dämpfung auf eine divergierende Amplitude im Resonanzpunkt. Es kann ein zusätzlicher linearer Dämpfungsterm hinzugefügt werden, was nach MEYER und NEUMANN [55] im akustischen Fall auf die gedämpfte Wellengleichung führt:

$$
\frac{1}{C_{1}^{2}} \frac{\partial^{2} \tilde{p}}{\partial t^{2}}-\Delta \tilde{p}+\frac{D}{C_{1}^{2} \varrho_{1}} \frac{\partial \tilde{p}}{\partial t}=0
$$

\section{$D \quad$ Dämpfungskonstante}

Für die zeitharmonische Betrachtung wird der komplexe Lösungsansatz gewählt:

$$
\tilde{p}=p_{\mathrm{A}}(\vec{x}) \mathrm{e}^{\imath \omega t}
$$

Mit diesem Ansatz folgt aus der gedämpften Wellengleichung (3.4) die gedämpfte Helmholtz-Gleichung:

$$
\left(\frac{\omega^{2}}{C_{1}^{2}}-\imath \frac{\omega D}{\varrho_{1} C_{1}^{2}}\right) p_{\mathrm{A}}+\Delta p_{\mathrm{A}}=0
$$

Die Dämpfung durch das Medium drückt sich in der komplexen Erweiterung der Wellenzahl aus:

$$
k^{2}=\frac{\omega^{2}}{C_{1}^{2}}-\imath \frac{\omega D}{\varrho_{1} C_{1}^{2}}
$$

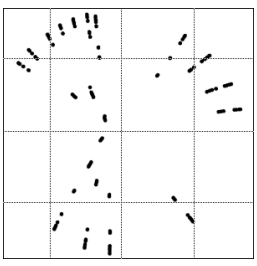


Neben Stehwellenfeldern werden später auch laufende Wellenfelder $\left(p_{\mathrm{A}}=\right.$ const. und $\nabla \varphi \neq 0$ ) in Anlehnung an das sich unter einer Sonotrode ausbildende Wellenfeld betrachtet (Abschnitt 3.4).

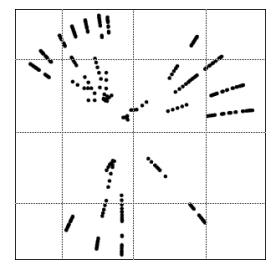




\title{
3.1 Analytische Beschreibung
}

Das sich in einer Küvette ausbildende Schallfeld ist von der Resonatorgeometrie und den Abschlüssen am Rand abhängig. Durch Reflexionen und Überlagerungen der fortwährend eingekoppelten Welle bilden sich frequenzabhängige Schalldruckverteilungen aus. Durch Verluste und Nichtlinearitäten bleiben die Amplituden in der Realität begrenzt. Hier wird der „eingeschwungene“ Zustand betrachtet, bei dem sich Energiezufuhr und Verluste die Waage halten und der Druck monofrequent mit zeitlich konstanter Amplitude oszilliert. Daher wird nur die HelmholtzGleichung gelöst, die die räumliche Verteilung der Druckamplitude beschreibt.

Eigenmoden des Systems stellen sich zu den Resonanzfrequenzen ein, dabei sind hier ideale (vollkommen reflektierende) Randbedingungen angenommen. Die exakten Modenformen können für einfache Fälle analytisch angegeben werden. Dies sind insbesondere Kugeln, Zylinder und Quader. So wird in Abbildung 3.1 ein quaderförmiger Resonator mit schallweichen Begrenzungen gezeigt, in dem sich eine $(1,1,1)$-Mode einstellt.

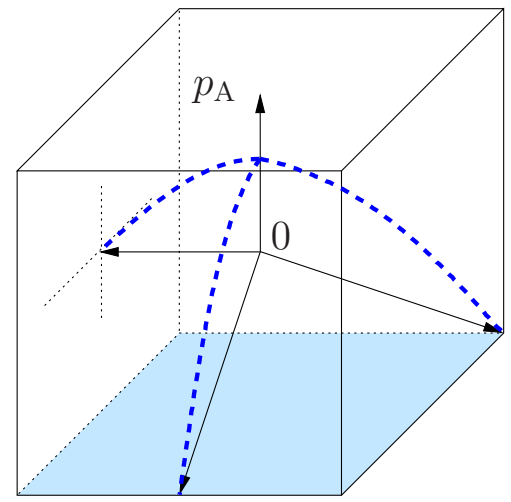

\begin{abstract}
Abbildung 3.1: Kubische Küvette mit schallweichen Seitenflächen. Skizziert ist schematisch der Verlauf der Schalldruckamplitude in der Grundmode vom Druckmaximum im Zentrum entlang der drei eingezeichneten Achsen zum Mittelpunkt der Seitenfläche und der Kante sowie zu einem Eckpunkt.
\end{abstract}

Die analytische mit $\omega=2 \pi \nu$ oszillierende Lösung der Helmholtz-Gleichung (3.3) für einen Quader mit den Kantenlängen $l_{\mathrm{x}}, l_{\mathrm{y}}, l_{\mathrm{z}}$ wird etwa von MEYER und NeUmann [55] angegeben:

$$
p_{\mathrm{A}}(\vec{x})=\hat{p}_{\mathrm{A}} \cos \left(k_{\mathrm{x}} x\right) \cos \left(k_{\mathrm{y}} y\right) \cos \left(k_{\mathrm{z}} z\right)
$$

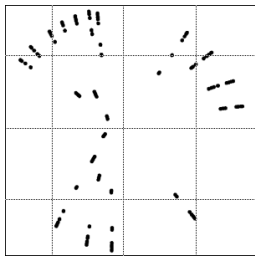




$$
\vec{x}=(x, y, z), \quad \vec{k}=\left(k_{\mathrm{x}}, k_{\mathrm{y}}, k_{\mathrm{z}}\right), \quad \lambda_{\mathrm{x}, \mathrm{y}, \mathrm{z}}=2 l_{\mathrm{x}, \mathrm{y}, \mathrm{z}}, \quad k_{\mathrm{x}, \mathrm{y}, \mathrm{z}}=\frac{2 \pi}{\lambda_{\mathrm{x}, \mathrm{y}, \mathrm{z}}}=\frac{\pi}{l_{\mathrm{x}, \mathrm{y}, \mathrm{z}}}
$$

$\lambda_{\mathrm{x}, \mathrm{y}, \mathrm{z}} \quad$ Wellenlänge des Schallfeldes in $x, y, z$-Richtung

$l_{\mathrm{x}, \mathrm{y}, \mathrm{z}} \quad$ Kantenlängen des Quaders

$\hat{p}_{\mathrm{A}} \quad$ maximale Schalldruckamplitude

$\vec{k} \quad$ Wellenzahlvektor

$p_{\mathrm{A}}$ ortsabhängige Amplitude des Schallfeldes

Diese analytische Lösung wurde in bisherigen Partikelmodellierungen der Strukturbildung von Kavitationsblasen zur Beschreibung einfacher Systeme verwendet (KoCH et al. [56]). Der nun folgende Ansatz zur numerischen Lösung erlaubt die Betrachtung gestörter Schallfeldmoden, etwa durch eingebrachte Objekte oder das Auftreten von Kavitationsblasen.

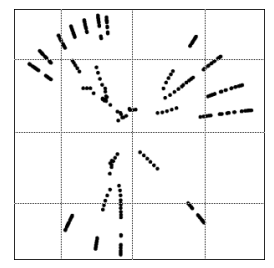




\subsection{Eigenmoden}

Die numerischen Simulationen in dieser Arbeit orientieren sich am Verhalten einfacher experimenteller Aufbauten. Für stehende Wellen, das heißt für den Fall eines Resonators, ist dies eine würfelförmige Glasküvette. Sie besitzt eine hohe Güte in Resonanz und wird nahe ihrer Grundmode betrieben.

Abbildung 3.2 zeigt das eingesetzte System bestehend aus der wasserbefüllten Küvette von $5 \times 5 \times 5 \mathrm{~cm}^{3}$, an die über die Bodenplatte ein ringförmiger, piezokeramischer Ultraschallwandler gekoppelt ist. Technische Details des Systems sind im Anhang B aufgeführt.

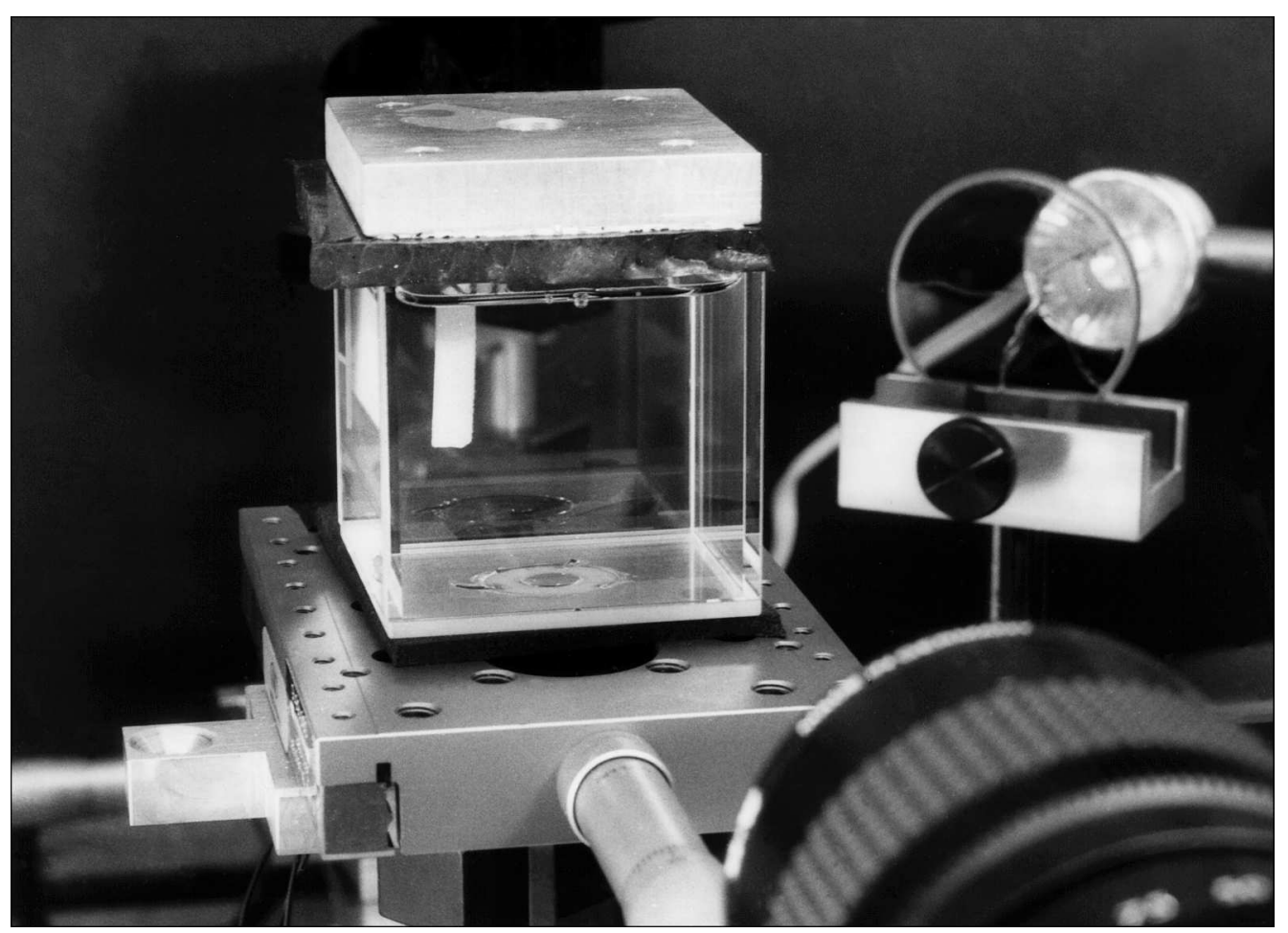

Abbildung 3.2: Aufnahme der quaderförmigen Küvette der Firma Hellma, Blickrichtung über das Kameraobjektiv, rechts hinten ist die Streulichtbeleuchtung zu sehen; Aufnahme entnommen aus Krefting [19].

Über den unter der Bodenfläche verklebten Piezoring wird das Ultraschallfeld in die Küvette eingekoppelt.

Zunächst wurde das System ohne Auftreten von Kavitation auf sein Resonanzverhalten untersucht. Die experimentellen Daten hierzu stammen aus der Arbeit von KREFTing [19].

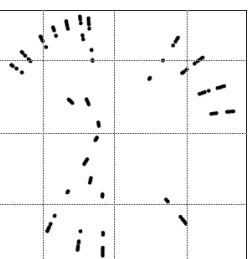


Die numerischen Untersuchung wurde mit FEMLAB ${ }^{1}$ [57] durchgeführt. Die Begrenzungsflächen und die freie Flüssigkeitsoberfläche werden dabei als schallweich angenommen $\left(p_{\mathrm{A}}=0\right)$. Die Beschleunigungen des Piezoringes werden durch die Bodenplatte auf das Fluid übertragen. Es bilden sich frequenzabhängige Schallfeldverteilungen im System aus.

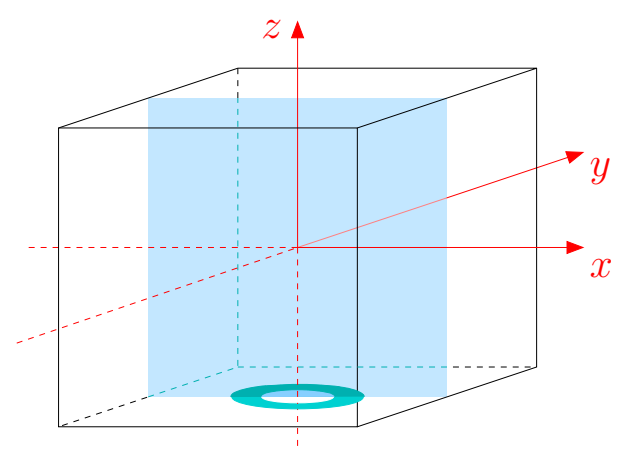

\begin{abstract}
Abbildung 3.3: Gezeigt ist das in der Diskussion verwendete Koordinatensystem. Die folgenden Abbildungen des Schallfeldes liegen in der $x$ - $z$-Ebene. Der Koordinatenursprung liegt im geometrischen Zentrum des Wasservolumens. In die Bodenplatte ist die das System treibende Piezofläche eingezeichnet.
\end{abstract}

Zur jeweiligen Resonanzfrequenz bildet sich die zugehörige Eigenmode aus. In der folgenden Arbeit werden diese Modenstrukturen in der in Abbildung 3.3 skizzierten $x$-z-Ebene $(y=0)$ dargestellt. Abbildung 3.4 zeigt die berechnete $(1,1,1)$-Mode für $\nu=23.647 \mathrm{kHz}$ sowie die $(1,1,2)$-Mode für $\nu=33.25 \mathrm{kHz}$.

Die Eigenfrequenz der (1, 1, 1)-Mode ist als Maxima in der Resonanzkurve (Abbildung 3.5) des Systems wiederzufinden. Die numerisch berechnete Lage der $(1,1,1)$-Resonanz verschiebt sich bereits mit einer angenommenen, um $1 \mathrm{~mm}$ verringerten Füllhöhe der Küvette um etwa $160 \mathrm{~Hz}$; Abbildung 3.5 (unten) zeigt diese Verschiebung für die beiden Füllhöhen $5 \mathrm{~cm}$ und $4.9 \mathrm{~cm}$. Damit deckt sich das numerische Resultat im Rahmen der Fehlertoleranz mit der im Experiment durch Vibrometermessungen von KREFTing [19] bestimmten Resonanz:

$$
\nu_{\text {res }}^{\text {num }} \approx 23.647 \mathrm{kHz} \quad \text { gegenüber } \quad \nu_{\text {res }}^{\text {exp }} \approx 23.85 \mathrm{kHz}
$$

Im ungedämpften System liefert die numerische Betrachtung eine scharfe Resonanzspitze. Durch Verwendung der gedämpften Helmholtz-Gleichung (3.5) und bei geeigneter Wahl des Dämpfungsterms lässt sich die Resonanzkurve in ihrer

${ }^{1}$ Programm zur numerischen Lösung von Differentialgleichung mittels der Methode der Finiten Elemente. 


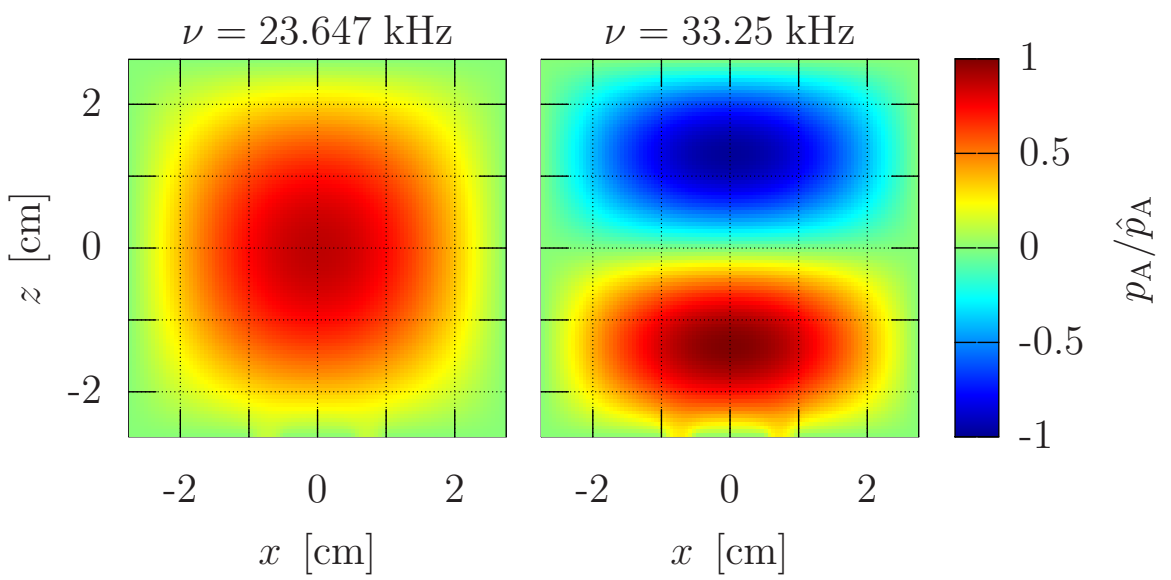

Abbildung 3.4: Die in der $x$-z-Ebene dargestellte, normierte Schalldruckverteilung ergibt sich als Lösung der Helmholtz-Gleichung (3.3) zu Eigenfrequenzen der quaderförmigen Hellma-Küvette mit Piezoring; $\omega=2 \pi \nu, C_{1}=1482 \mathrm{~m} / \mathrm{s}$; links: $(1,1,1)$-Mode bei $\nu=23.647 \mathrm{kHz}$; rechts: $(1,1,2)$-Mode bei $\nu=33.25 \mathrm{kHz}$.

Halbwertsbreite an die experimentell gemessenen Verläufe anpassen. Von KREFTING [19] wird nicht der absolute Schalldruck, sondern die Wandgeschwindigkeit der Küvette gemessen. Für hohe Amplituden, noch im Bereich des linearen Zusammenhanges der Wandgeschwindigkeit mit der Schalldruckamplitude, wird die Eichung angegeben:

$$
5.5 \mathrm{kPa} \mathrm{s} / \mathrm{mm}, \quad p_{\mathrm{A}} \approx 40 \mathrm{kPa}
$$

Die Breite der Resonanzspitze ist ein Maß für die Güte des Systems. Mit zunehmender Dämpfung verbreitert sich die Resonanz und ihre Höhe nimmt ab. Außerdem verschiebt sie sich leicht zu niedrigeren Frequenzen (entsprechende Darstellungen geben Meyer und Guicking [58]). Die in der Vibrometermessung bestimmte Halbwertsbreite beträgt etwa:

$$
\Delta_{\mathrm{hwb}}^{\exp } \approx 90 \mathrm{~Hz}
$$

Im numerischen Fall wird die gedämpfte Wellengleichung (3.5) zur Bestimmung der Resonanzkurve herangezogen. Der ungedämpfte Fall führt bei genauem Einstellen der Resonanzfrequenz zu einem beliebigen Ansteigen der Druckamplitude. Über den Dämpfungsfaktor $D$ lässt sich nun die numerisch bestimmte Halbwertsbreite an den von KREFTing [19] gemessenen Verlauf anpassen, wie in Abbildung 3.5 gezeigt. Die den gewählten Dämpfungskonstanten entsprechenden Halbwertsbreiten stellt Tabelle 3.1 zusammen. Die Halbwertsbreite skaliert linear mit der Dämpfungskonstanten, die experimentelle Halbwertsbreite von

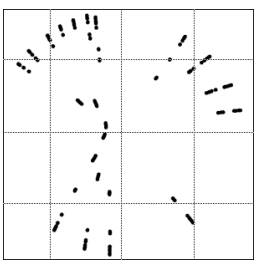




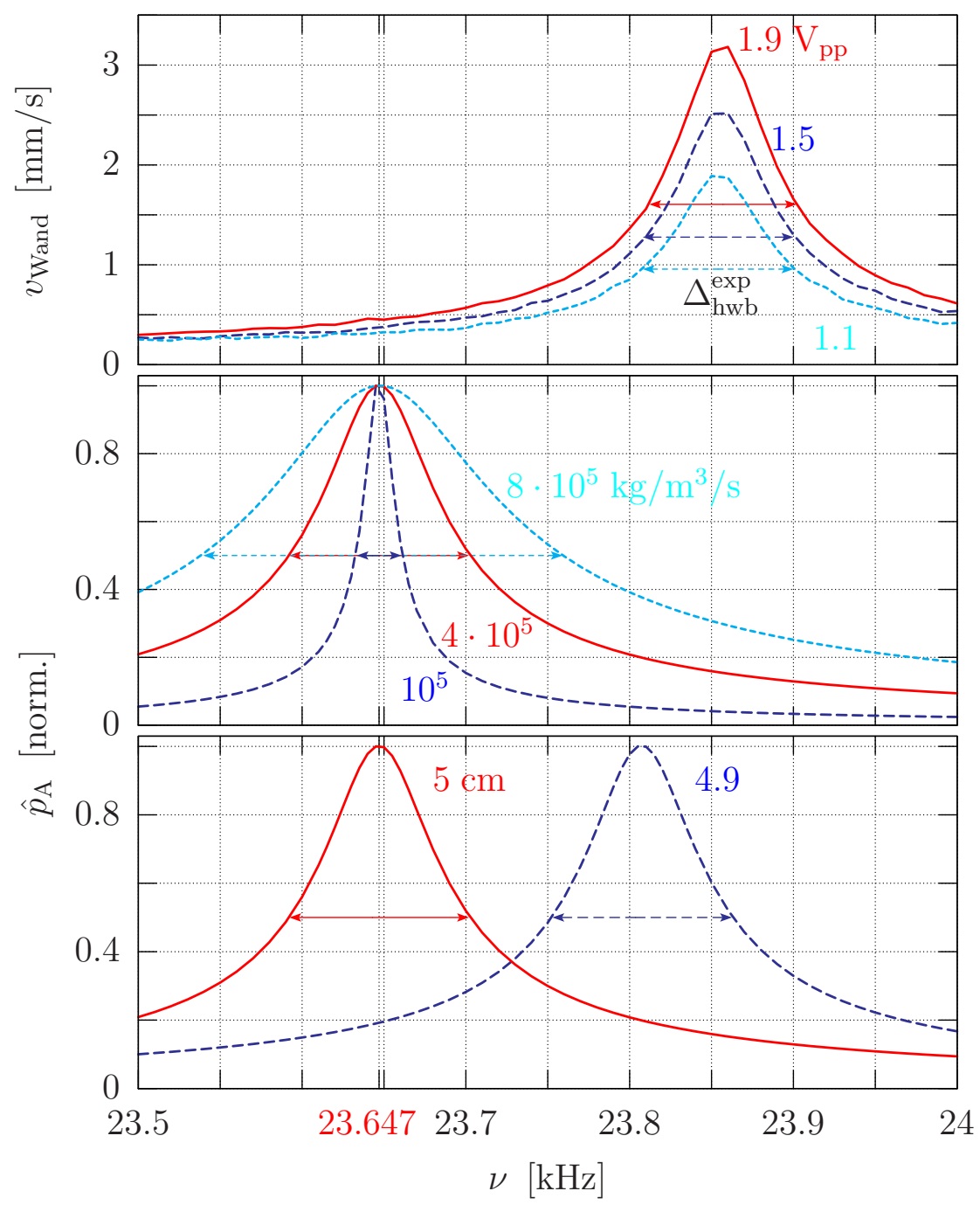

Abbildung 3.5: Resonanzkurven der Hellma-Küvette mit Piezoring; Oben: Vibrometermessung an der Quaderwand zu unterschiedlichen Anregungsamplituden. Die Messungen wurden in der blasenfreien Küvette von Krefting [19] durchgeführt. Es wird angenommen, dass ein linearer Zusammenhang zwischen Küvettenwandgeschwindigkeit $v_{\text {Wand }}$ und der Druckamplitude sowie zwischen Anregungsspannung des Piezoringes und seiner Auslenkung besteht. Mitte: numerische Lösung der gedämpften Helmholtz-Gleichung (3.5); schallweiche Abschlüsse, ohne Blasen. Unten: Resonanzverschiebung durch Variation der Füllhöhe der Küvette für $5 \mathrm{~cm}$ und $4.9 \mathrm{~cm} ; \varrho_{1}=998 \mathrm{~kg} / \mathrm{m}^{3}, C_{\mathrm{l}}=1482 \mathrm{~m} / \mathrm{s}, D=$ $10^{5}, 4 \cdot 10^{5}, 8 \cdot 10^{5} \mathrm{~kg} / \mathrm{m}^{3} / \mathrm{s}$.

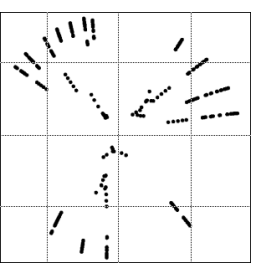


$90 \mathrm{~Hz}$ ergibt sich aus der numerischen Lösung gerade für eine Dämpfung von $D=3.26 \cdot 10^{5} \mathrm{~kg} / \mathrm{m}^{3} / \mathrm{s}$.

\begin{tabular}{cc}
$D\left[10^{5} \mathrm{~kg} / \mathrm{m}^{3} / \mathrm{s}\right]$ & $\Delta_{\mathrm{hwb}}^{\text {num }}[\mathrm{Hz}]$ \\
\hline 1 & 27 \\
3.26 & $90 \approx \Delta_{\mathrm{hwb}}^{\exp }$ \\
4 & 109 \\
8 & 219
\end{tabular}

Tabelle 3.1: Aufstellung der numerisch ermittelten Halbwertsbreiten für die in Abbildung 3.5 gezeigten Resonanzkurven.

Die wirkliche Wellendämpfung in Wasser ist vergleichbar klein und kann vernachlässigt werden ${ }^{2}$, so dass durch die Anpassung des Dämpfungsfaktors praktisch allein die Güte des Resonators ausgedrückt wird. Im experimentellen System wird ein Teil der Verlustenergie durch die nicht idealen Reflexionen akustisch abgestrahlt und vermutlich der Hauptteil in Wandschwingungen komplizierterer Art umgesetzt und dort absorbiert. Im Modell wird das durch den überall gleichmäßig im Wasser wirkenden Dämpfungsterm modelliert, da diese Methode am einfachsten zu realisieren ist.

Mit Variation des Dämpfungsterms ändert sich die Lage der Resonanz. Diese Verschiebung ist in Abbildung 3.6 aufgetragen. Wird als die konstante treibende Frequenz die Resonanzfrequenz des blasenfreien ungedämpften Systems gewählt, so kommt es bei hinreichender Dämpfung zu einem Modenübergang von der $(1,1,1)$ - auf die $(1,1,2)$-Mode.

Für das blasenfreie System, für das nach Abbildung 3.5 ein Dämpfungsfaktor von $D=3.26 \cdot 10^{5} \mathrm{~kg} / \mathrm{m}^{3} / \mathrm{s}$ angepasst wurde, tritt keine signifikante Resonanzverschiebung in Erscheinung. Ein deutlicher Verschiebungseffekt ist erst ab einem Dämpfungsfaktor von etwa $10^{7} \mathrm{~kg} / \mathrm{m}^{3} / \mathrm{s}$ zu erkennen.

2 Meyer und Neumann [55] geben an, dass eine $10 \mathrm{kHz}$ Welle in Wasser $100 \mathrm{~km}$ laufen muss, um um $1 \mathrm{~dB}$ bzw. $12 \%$ in der Amplitude gedämpft zu werden. 


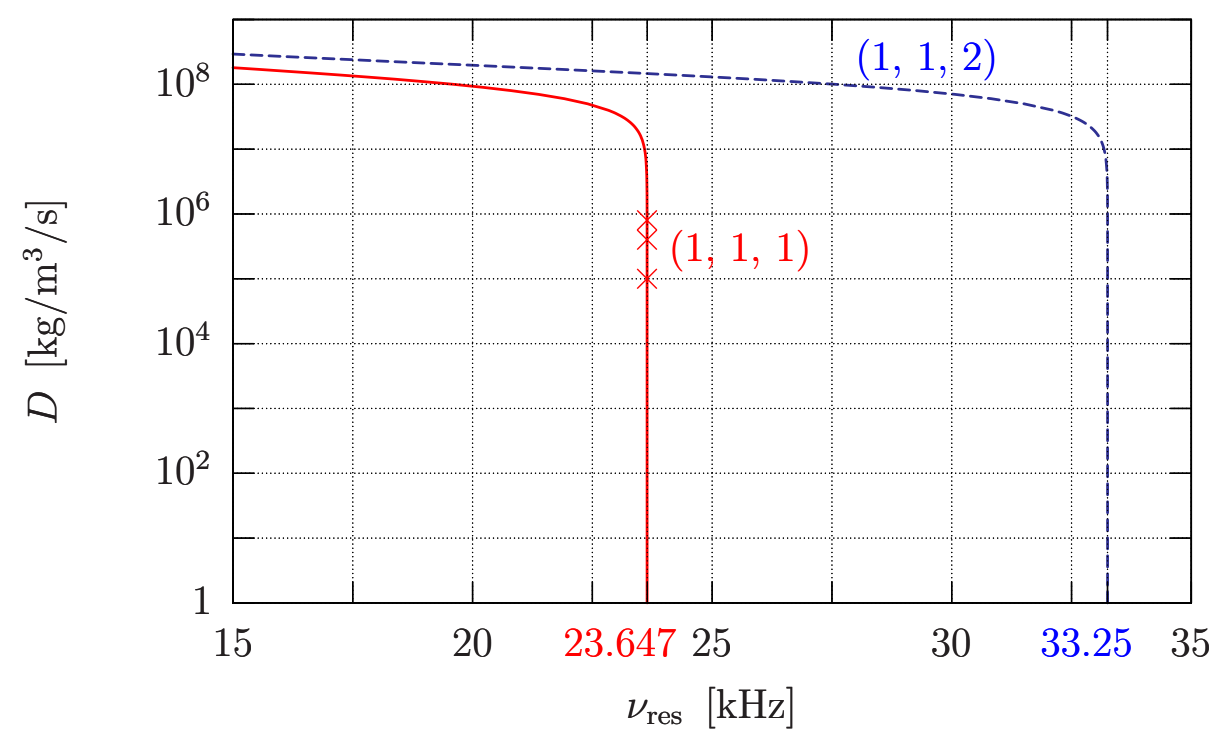

Abbildung 3.6: Betrachtete Verschiebung des Resonanzspektrums nach (3.5) der kavitationsfreien Hellma-Küvette mit Piezoring abhängig von dem Dämpfungsfaktor. Eingezeichnet ist die Abhängigkeit der Resonanzfrequenz der (1, 1, 1)- sowie der (1, 1, 2)-Mode; Die Kreuze zeigen die in Abbildung 3.5 betrachteten Parameter an; $\varrho_{1}=998 \mathrm{~kg} / \mathrm{m}^{3}, C_{\mathrm{l}}=$ $1482 \mathrm{~m} / \mathrm{s}$.

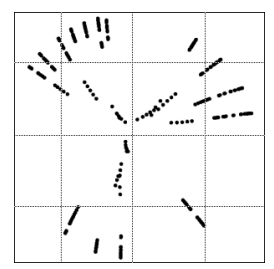




\subsection{Blasen-Schallfeld-Wechselwirkung}

Befinden sich Blasen in der Flüssigkeit, so ändern sich die akustischen Eigenschaften des Resonatorsystems. Ein erhöhter Blasenanteil führt in der Regel zu einer Absenkung der Schallgeschwindigkeit in dem entsprechenden Volumen, was wiederum die Resonanz des Systems verstimmt. Diese Rückkopplung von Kavitationsblasen auf die Druckverteilung im Resonator wird theoretisch im Folgenden unter Annahme eines Blasen-Flüssigkeits-Gemisches untersucht.

Um die Ausbreitung akustischer Wellen in blasenhaltigen Flüssigkeiten zu beschreiben, leitet VAN WIJNGAARDEN [59] heuristisch eine modifizierte Wellengleichung ab. Die Verknüpfung von Gas- und Wasser-Anteilen in dem Medium gelingt durch die Einführung einer zeit- und ortsabhängigen Dichte des Gemisches:

$$
\varrho_{\mathrm{m}}=\varrho_{\mathrm{m}}(\vec{x}, t)
$$

Ausgehend von der bekannten Ableitung der Wellengleichung über Kontinuitätsund Euler-Gleichung zeigen Commander et al. [60] ebenfalls eine Ableitung der modifizierten Wellengleichung wie sie von van Wijngaarden angegeben wird. Über mikroskopische Gleichungen konnten CAFLISCH et al. [61] das Modell von van Wijngaarden streng herleiten. Dabei wird die Wellenlänge einer sich im Gemisch ausbreitenden Störung als groß gegenüber typischen Blasenradien angenommen $\left(\lambda \gg R_{\mathrm{n}}\right)$, und der Gasanteil als sehr klein vorausgesetzt $(\alpha \ll 1)$.

\subsubsection{Wijngaarden-Modell}

Das Modellsystem von van Wijngaarden setzt sich zusammen aus der divergenzfreien Kontinuitätsgleichung $\left(\nabla \cdot \varrho_{\mathrm{l}}=0\right)$, der Euler-Gleichung, der Dichte des Gemisches, der Erhaltung der Blasenanzahl, der adiabatischen Zustandsgleichung für das Gas und der Blasendynamik nach Keller-Miksis ${ }^{3}$ (2.10):

$$
\begin{aligned}
\frac{1}{\varrho_{\mathrm{l}}} \frac{\partial \varrho_{\mathrm{l}}}{\partial t}+\nabla \cdot \vec{u} & =0 \\
\varrho_{1}\left(\frac{\partial \vec{u}}{\partial t}+\vec{u} \cdot \nabla \vec{u}\right)+\nabla \tilde{p} & =0 \\
\varrho_{\mathrm{m}} & =\varrho_{\mathrm{l}}(1-\alpha)
\end{aligned}
$$

3 Van Wijngaarden verwendet ursprünglich das Rayleigh'sche Blasenmodell. In dieser Arbeit wird das realistischere Keller-Miksis-Modell eingesetzt. 


$$
\begin{aligned}
\frac{\mathrm{d} n}{\mathrm{~d} t}+\nabla \cdot(n \vec{u}) & =0 \\
p_{\mathrm{g}} R^{3 \eta} & =\text { const. } \\
\left(1-\frac{\dot{R}}{C_{\mathrm{l}}}\right) R \ddot{R}+\frac{3}{2}\left(1-\frac{\dot{R}}{3 C_{\mathrm{l}}}\right) \dot{R}^{2} & =\left(1+\frac{\dot{R}}{C_{\mathrm{l}}}\right) \frac{p}{\varrho_{\mathrm{l}}}+\frac{R}{\varrho_{\mathrm{l}} C_{\mathrm{l}}} \frac{\mathrm{d} p}{\mathrm{~d} t}
\end{aligned}
$$

Der Gasdruck in der Blase ist nach (2.11) anzusetzen:

$$
p_{\mathrm{g}}=p_{\mathrm{stat}}-p_{\mathrm{v}}+\frac{2 \sigma}{R_{\mathrm{n}}}
$$

Im Folgenden wird der Aufbau dieses Modells dargelegt und die Wellengleichung zur Beschreibung einer sich ausbreitenden Druckstörung im Blasen-FluidGemisch abgeleitet.

Die Dichte des Gemisches setzt sich entsprechend dem Gas- bzw. Flüssigkeitsanteil aus den jeweiligen Dichten zusammen:

$$
\varrho_{\mathrm{m}}=(1-\alpha) \varrho_{\mathrm{l}}+\alpha \varrho_{\mathrm{g}}
$$

$\begin{array}{cl}\varrho_{\mathrm{m}} & \text { Dichte des Gemisches } \\ \varrho_{\mathrm{l}} & \text { Dichte der Flüssigkeit } \\ \varrho_{\mathrm{g}} & \text { Dichte des Gases }\end{array}$

$\alpha \in[0,1] \quad$ Gasanteil im Volumen („volume fraction“)

Da die Dichte des Gases deutlich unter der Dichte des Fluids liegt $\left(\varrho_{\mathrm{g}} \ll \varrho_{\mathrm{l}}\right)$ und der Gasanteil am Gemisch klein angenommen werden soll $(\alpha \ll 1)$, gilt für die Dichte des Gemisches die in (3.7c) angegebene Näherung:

$$
\varrho_{\mathrm{m}} \approx(1-\alpha) \varrho_{1}
$$

Werden ausschließlich ideal sphärische Blasen eines Ruheradius angenommen, so kann der Gasanteil ausgedrückt werden über die Blasenzahldichte $n=n(\vec{x}, t)$ multipliziert mit ihrem Volumenverlauf:

$$
\alpha=n V=n \frac{4}{3} \pi R^{3}
$$

$$
\begin{aligned}
n=n(\vec{x}, t) & \text { Blasen pro Volumen } \\
V & \text { zeitabhängiges Volumen einer Blase }
\end{aligned}
$$

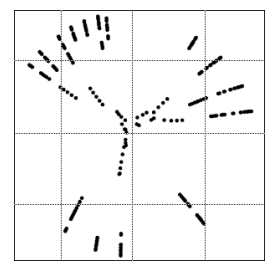


Der Gasanteil in einem Fluidvolumen ändert sich mit der Blasendichte $n=n(\vec{x}, t)$ und der Oszillationsdynamik der Blase $R=R\left(t, p_{\mathrm{A}}, R_{\mathrm{n}}\right)$ :

$$
\frac{\mathrm{d} \alpha}{\mathrm{d} t}=4 \pi R^{2} \dot{R} n+\frac{4}{3} \pi R^{3} \dot{n}
$$

Blasenerhaltung nach (3.7d) liegt vor, sofern Fragmentierungen, Kollisionen von Blasen und ihre Erzeugung oder Auflösung vernachlässigt werden. Die Gesamtblasenzahl ist dann eine Erhaltungsgröße ( $N=$ const.), deren lokale zeitliche Änderung gerade dem Blasenfluss durch ein betrachtetes quellen- und senkenfreies Volumen entspricht $(\nabla \cdot n=0)$ :

$$
\frac{\mathrm{d} n}{\mathrm{~d} t}+n \nabla \cdot \vec{u}_{\mathrm{B}}=0
$$

$\vec{u}_{\mathrm{B}} \quad$ Translationsgeschwindigkeit der Blasen

Die Kontinuitätsgleichung (3.7a) schreibt sich für ein Gemisch als:

$$
\frac{\partial \varrho_{\mathrm{m}}}{\partial t}+\nabla \cdot\left(\varrho_{\mathrm{m}} \vec{u}\right)=0
$$

$\vec{u} \quad$ Strömungsgeschwindigkeit des Gemisches

Durch Einsetzen der Näherung (3.7c) für die Gemischdichte folgt:

$$
\frac{1}{\varrho_{1}} \frac{\partial \varrho_{1}}{\partial t}+\nabla \cdot \vec{u}+\underbrace{\frac{1}{\varrho_{1}} \vec{u} \nabla \cdot \varrho_{1}}_{=0}=\frac{1}{1-\alpha}\left(\frac{\partial \alpha}{\partial t}+\vec{u} \nabla \cdot \alpha\right)
$$

Der Divergenzterm für die Flüssigkeitsdichte verschwindet im Fall einer inkompressiblen Flüssigkeit ( $\varrho_{1}=$ const.), ortsabhängig ist lediglich die Dichte des Gemisches $\varrho_{\mathrm{m}}$. Auf der rechten Seite lässt sich der Klammerausdruck als die totale Zeitableitung von $\alpha$ auffassen:

$$
\frac{1}{\varrho_{1}} \frac{\partial \varrho_{1}}{\partial t}+\nabla \cdot \vec{u}=\frac{1}{1-\alpha} \frac{\mathrm{d} \alpha}{\mathrm{d} t}
$$

Die totale zeitliche Ableitung des Gasanteils $\alpha$ wird durch (3.9) ausgedrückt, die hier auftretende Ableitung der Blasenzahldichte $n$ wird durch die Erhaltungsgleichung für die Blasenzahl (3.10) ersetzt:

$$
\frac{\mathrm{d} \alpha}{\mathrm{d} t}=4 \pi R^{2} \dot{R} n+\frac{4}{3} \pi R^{3} \cdot \overbrace{\left(-n \nabla \cdot \vec{u}_{\mathrm{B}}\right)}^{\dot{n}=}, \quad \alpha=\frac{4}{3} \pi R^{3} n
$$

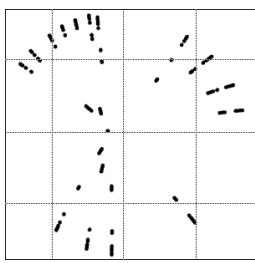


Dieser Ausdruck wird in (3.11) eingesetzt. Die Geschwindigkeit des Blasenfeldes soll bei der Berechnung des Schallfeldes der Strömungsgeschwindigkeit des Fluids entsprechen: $\overrightarrow{u_{\mathrm{B}}} \approx \vec{u}$. Die Relativbewegung von Blasen und Flüssigkeit wird hier also vernachlässigt. Der Gasanteil im Fluid wird als sehr niedrig angenommen $(\alpha \ll 1)$, womit gilt $1 \pm \alpha \approx 1$ :

$$
\begin{aligned}
\frac{1}{\varrho_{1}} \frac{\partial \varrho_{1}}{\partial t}+\nabla \cdot \vec{u} & =\underbrace{\frac{1}{1-\alpha}}_{\approx 1}\left(4 \pi R^{2} \dot{R} n-\alpha \nabla \cdot \vec{u}\right) \\
\Rightarrow \quad \frac{1}{\varrho_{1}} \frac{\partial \varrho_{1}}{\partial t}+\underbrace{(1+\alpha)}_{\approx 1} \nabla \cdot \vec{u} & =4 \pi R^{2} \dot{R} n
\end{aligned}
$$

An dieser Stelle soll eine zeitunabhängige Blasendichte angenommen werden $(n=$ $n(\vec{x})$ ). Dies entspricht einer strömungsfreien Flüssigkeit und der Betrachtung von Zeitskalen unterhalb der Blasentranslation.

Die sich aus (3.7b) ergebende linearisierte Euler-Gleichung lässt sich auf Gemische anwenden in der Form:

$$
-\nabla \tilde{p}=\varrho_{\mathrm{m}} \frac{\partial \vec{u}}{\partial t}
$$

Unter der Annahme eines niedrigen Gasanteils im Fluid $(\alpha \ll 1)$ wird die Gemischdichte genähert durch:

$$
\varrho_{\mathrm{m}}=\varrho_{1} \underbrace{(1-\alpha)}_{\approx 1} \approx \varrho_{1}
$$

Entsprechend wird die Euler-Gleichung zur Flüssigkeits-Euler-Gleichung:

$$
-\nabla \tilde{p}=\varrho_{1} \frac{\partial \vec{u}}{\partial t}
$$

Aus diesem Gleichungssystem wird die Wellengleichung im Gas-FlüssigkeitsGemisch abgeleitet. Die zeitliche Ableitung der Kontinuitätsgleichung (3.12) führt auf:

$$
\frac{1}{\varrho_{1}} \frac{\partial^{2} \varrho_{1}}{\partial t^{2}}+\nabla \cdot \frac{\partial \vec{u}}{\partial t}=4 \pi n\left(2 R \dot{R}^{2}+R^{2} \ddot{R}\right)
$$

$n$ zeitunabhängige Blasenzahldichte

Einsetzen der linearisierten Euler-Gleichung (3.13) liefert:

$$
\frac{\partial^{2} \varrho_{1}}{\partial t^{2}}-\nabla \cdot \nabla \tilde{p}=\varrho_{1} 4 \pi n\left(2 R \dot{R}^{2}+R^{2} \ddot{R}\right)
$$

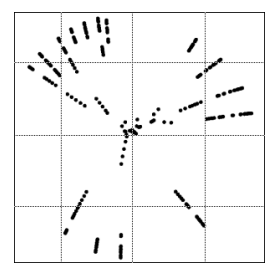


Von der adiabatischen Zustandsgleichung (3.7e) ausgehend, leiten beispielsweise Cremer und Möser [62] den um den Gleichgewichtspunkt $\left(p_{\text {stat }}, \varrho_{1}\right)$ linearisierten Zusammenhang von Schalldruck und Flüssigkeitsdichte ab:

$$
\varrho_{1}=\frac{\tilde{p}}{C_{1}^{2}}
$$

Da Änderungen der Gemischdichte gegenüber der Flüssigkeitsdichte für niedrigen Gasgehalt $(\alpha \ll 1)$ vernachlässigt werden können, geht eine Druckänderung im Wesentlichen mit einer Änderung in der Schallgeschwindigkeit einher.

Die Wijngaarden-Wellengleichung gekoppelt an das Blasenfeld lautet dann:

$$
\frac{1}{C_{1}^{2}} \frac{\partial^{2} \tilde{p}}{\partial t^{2}}-\Delta \tilde{p}=\varrho_{1} 4 \pi n\left(2 R \dot{R}^{2}+R^{2} \ddot{R}\right)
$$

Diese Gleichung beschreibt mit geeigneten Randbedingungen die Wellenausbreitung in schwach blasenhaltigen Flüssigkeiten unter den angenommenen Näherungen und ist Basis für die Betrachtung der Rückkopplung der Blasen auf das Schallfeld.

Eine zeitliche Veränderung der Blasendichte $n(\vec{x}, t)$ wird in dem folgenden Kapitel durch das Partikelmodell eingeführt (Abschnitt 4.5). Verschiedene Autoren betrachten auch direkt eine Evolutionsgleichung für die kontinuierliche Größe $n(\vec{x}, t)$, so zum Beispiel Akhatov et al. [63], Kobelev et al. [64] oder SERvant et al. [65]. Diese Ansätze konnten aber bisher die Blasenwechselwirkung nicht hinreichend berücksichtigen, so dass in der vorliegenden Arbeit der neue Weg der Partikelmodellierung gewählt wird.

\subsubsection{Numerische Lösungen im Eindimensionalen}

Die volle Gleichung (3.14) wurde in FEMLAB [57] für die eindimensionale Wellenausbreitung implementiert. Der linke Rand stellt dabei eine harmonisch abstrahlende Quelle dar, der rechte Rand ist ideal schallweich reflektierend. Die gewählten Abmessungen sind hier in Anlehnung an die äußere Kantenlänge der aus Abschnitt 3.2 bekannten Hellma-Küvette von $l_{\mathrm{x}}=5.5 \mathrm{~cm}$ gewählt. Die Anregungsfrequenz ist entsprechend angepasst für die Ausbildung eines Druckbauches:

$$
\nu=\frac{C_{\mathrm{l}}}{l_{\mathrm{x}}} \approx 13.47 \mathrm{kHz}
$$

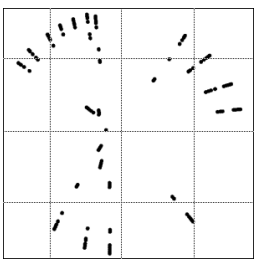


Transiente Lösungen der Wijngaarden-Wellengleichung (3.14) sind in Abbildung 3.7 gezeigt. Berechnet sind sie für nichtlineare, nicht zu stark getriebene Blasenpulsationen, beschrieben durch das Keller-Miksis-Modell (2.10). Es ist eine deutliche Beeinflussung der Schallgeschwindigkeit in Abhängigkeit von der Blasenzahldichte zu erkennen. Die Blasenzahlverteilung wird als symmetrische Gaußverteilung um den Ursprung modelliert:

$$
\operatorname{gauss}(\vec{x})=\frac{n_{\max }}{(2 \pi)^{3 / 2} \Delta^{3}} \mathrm{e}^{-\vec{x}^{2} /\left(2 \Delta^{2}\right)}
$$

$\Delta$ Parameter der Verteilungsbreite

$n_{\max }$ Skalierungsfaktor

Die Halbwertsbreite dieser Verteilung ergibt sich aus dem beispielsweise von Bronstein et al. [47] angegebenen Zusammenhang:

$$
\Delta_{\mathrm{hwb}}=2 \sqrt{2 \log (2)} \Delta \approx 2.35 \Delta
$$

Die Gesamtzahl der Blasen in dem betrachteten Volumen $\left(l_{\mathrm{x}}, l_{\mathrm{y}}, l_{\mathrm{z}}\right)$ ergibt sich durch die Integration der Verteilungsfunktion (3.15):

$$
N=n_{\max } \operatorname{erf}\left(\frac{l_{\mathrm{x}}}{2 \sqrt{2} \Delta}\right) \operatorname{erf}\left(\frac{l_{\mathrm{y}}}{2 \sqrt{2} \Delta}\right) \operatorname{erf}\left(\frac{l_{\mathrm{z}}}{2 \sqrt{2} \Delta}\right)
$$

Die Gauß'sche Fehlerfunktion erf $(x)$ ist laut Bronstein et al. [47] definiert als:

$$
\operatorname{erf}(x) \equiv \frac{2}{\sqrt{\pi}} \int_{0}^{x} \mathrm{e}^{-t^{2}} \mathrm{~d} t
$$

In dem hier relevanten Parameterbereich von $l_{\mathrm{x}, \mathrm{y}, \mathrm{z}} \approx 5 \mathrm{~cm}$ und $\Delta_{\mathrm{hwb}} \leq 2 \mathrm{~cm}$ geht die Fehlerfunktion gegen Eins und kann im Folgenden vernachlässigt werden:

$$
N \approx n_{\max }
$$

Für die Übertragung des Begriffs der Blasenzahldichte in das Eindimensionale geht die Gesamtzahl an Blasen $N$ über in eine Flächendichte an Blasen $N_{\text {yz }}$. Diese Flächendichte ist konstant in der Ebene, senkrecht zu der betrachteten Achse.

In den Auftragungen von Abbildung 3.7 sind für verschiedene Blasenzahldichteverteilungen eindimensionale Lösungen der Wijngaarden-Gleichung (3.14) gezeigt. Die am linken Rand $(x=-2.75 \mathrm{~cm})$ mit fester Frequenz $\nu=13.47 \mathrm{kHz}$

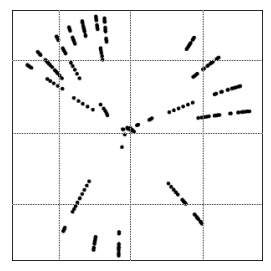



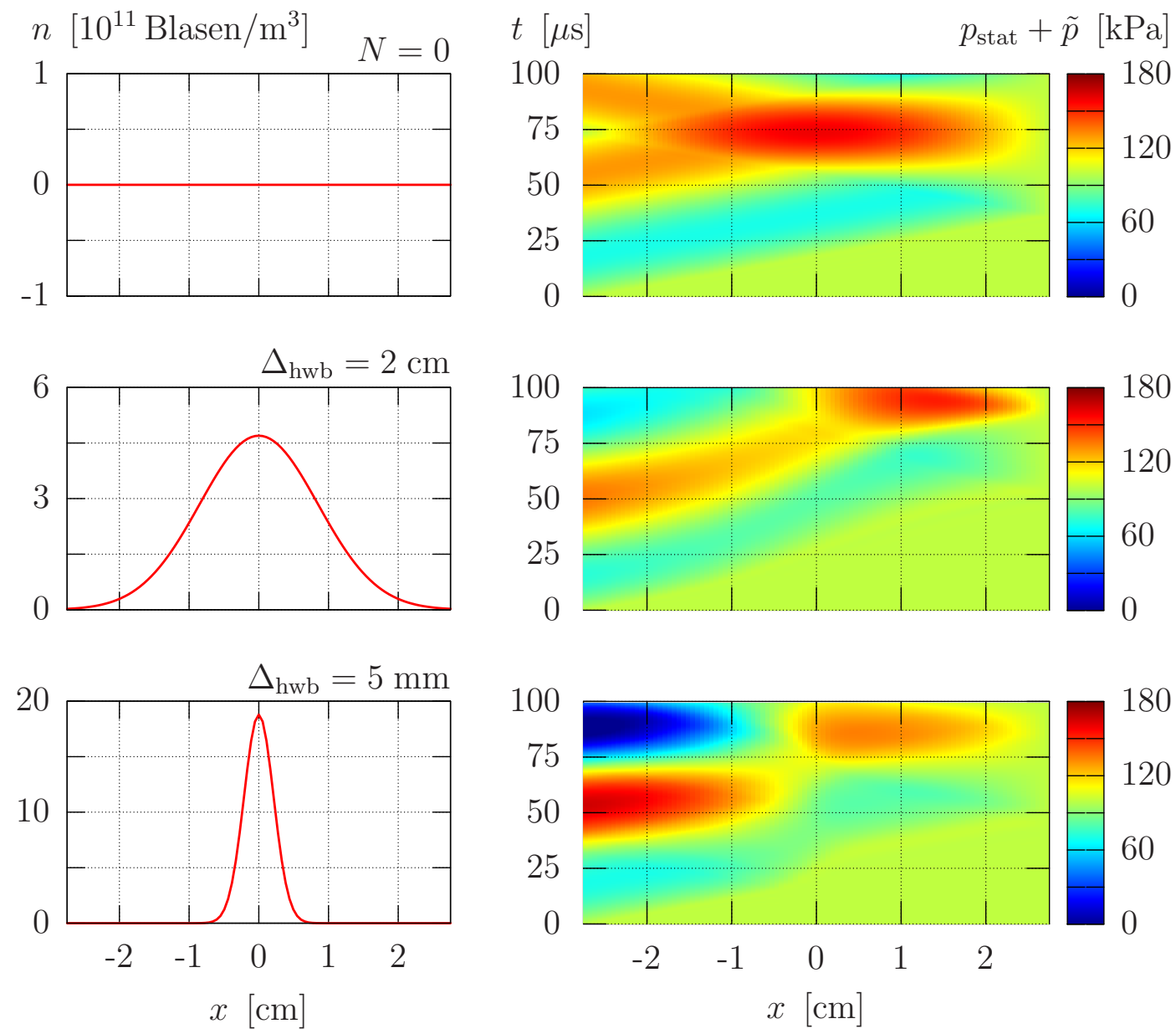

Abbildung 3.7: $\mathrm{Zu}$ unterschiedlichen Blasenzahlverteilungen ist die zeitliche Entwicklung des Druckes als Lösung der Wijngaarden-Gleichung (3.14) gezeigt. Die von links einlaufende Welle wird schallweich reflektiert und überlagert sich mit dem nachfolgenden Wellenzug. Farbkodiert ist die raum-zeitliche Entwicklung des Druckes $p_{\text {stat }}+\tilde{p}$ aufgetragen. Oben sind keine Blasen vorhanden, in der Mitte und unten ist die Flächendichte $N_{\text {yz }}=10^{10}$ Blasen $/ \mathrm{m}^{2}$. Zu erkennen ist die Verlangsamung des Wellenzuges in der Region hoher Blasendichte; $\nu=13.47 \mathrm{kHz}, R_{\mathrm{n}}=5 \mu \mathrm{m}$, $p_{\mathrm{A}}=30 \mathrm{kPa}, p_{\text {stat }}=100 \mathrm{kPa}, \Delta_{\mathrm{hwb}}=2 \mathrm{~cm}, 5 \mathrm{~mm}$.

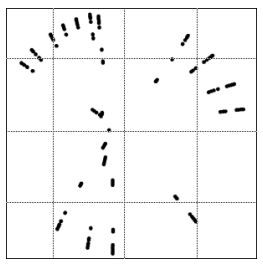


eingekoppelte Welle läuft durch den Resonator und wird am rechten Abschluss $(x=2.75 \mathrm{~cm})$ schallweich reflektiert. Durch die Überlagerung der reflektierten mit der einlaufenden Welle bildet sich über die Zeit ein Stehwellenfeld aus, das im ungedämpften Fall kontinuierlich in der Amplitude anwächst.

Die Rand- und Anfangsbedingungen sind gegeben als:

$$
\tilde{p}_{\text {links }}=p_{\mathrm{A}} \sin (2 \pi \nu t) ; \quad \tilde{p}_{\text {rechts }}=0 ; \quad \tilde{p}(t=0)=0
$$

Die oberen Auftragungen in Abbildung 3.7 zeigen die Situation unterhalb der Kavitationsschwelle. Die Blasenzahldichte ist hier Null und damit die Schallgeschwindigkeit über den Raum konstant, wie an der gleichförmig einlaufenden Welle in der Raum-Zeit-Ebene zu sehen ist. Über die Zeit bildet sich der einzelne Druckbauch der Grundmode aus.

Mit der ausgedehnten gaußförmigen Blasenzahldichteverteilung (Mitte) nimmt die Schallgeschwindigkeit um den Mittelpunkt ab. Entsprechend werden in den Ursprung einlaufende Wellen verzögert und auslaufende Wellen beschleunigt.

Eine schmalere Verteilung der Blasenzahldichte um den Mittelpunkt (unten) bewirkt eine relativ sprunghafte Änderung der Schallgeschwindigkeit. Mit steigender Blasenkonzentration um das Zentrum wirkt dieser Bereich zunehmend als schallweiche Trennung zwischen dem sich in der linken Hälfte ausbildenden Feld und dem schwächeren Feld in der rechten Hälfte, das heißt an der „Blasenwand“ wird bei hoher Blasendichte die Welle stark reflektiert. Dieser Effekt zeigt sich auch an der geringeren Stehwellenamplitude rechts der Mitte.

\subsubsection{Linearisierung}

Es zeigt sich, dass mit FEMLAB [57] die nichtlineare Blasenschwingung nur bis zu moderaten Druckamplituden gerechnet werden kann. Für zu große Amplituden, bzw. zu heftigen Blasenkollaps, treten numerische Fehler auf. Mit der folgenden Vereinfachung des Modells durch Einsetzen der linearisierten Keller-MiksisGleichung (2.12) wird die numerische Betrachtung stärker getriebener radialer Blasenpulsationen möglich $\left(p_{\mathrm{A}}>30 \mathrm{kPa}\right)$. Dabei wird in Kauf genommen, dass das Schwingungsverhalten der Blasen nicht mehr realistisch modelliert wird. Um mit dem Programmpaket FEMLAB die volle nichtlineare Gleichung zu behandeln, müsste der Differentialgleichungslöser modifiziert werden, was zu großen Aufwand erfordert hätte. 
Zur Linearisierung im Radius wird verwendet:

$$
R(t)=R_{\mathrm{n}}+\tilde{R}(t) \quad \Rightarrow \quad \dot{R}(t)=\dot{\tilde{R}}(t) \quad \Rightarrow \quad \ddot{R}(t)=\ddot{\tilde{R}}(t)
$$

Damit wird die rechte Seite der Wijngaarden-Gleichung (3.14) ausgedrückt und in linearer Näherung nur Terme bis zur Größenordnung $\mathcal{O}(\tilde{R})$ berücksichtigt:

$$
\begin{aligned}
\varrho_{1} 8 \pi n R \dot{R}^{2}+\varrho_{1} 4 \pi n R^{2} \ddot{R} & =\varrho_{1} 8 \pi n\left(R_{\mathrm{n}}+\tilde{R}\right) \dot{\tilde{R}}^{2}+\varrho_{1} 4 \pi n\left(R_{\mathrm{n}}+\tilde{R}\right)^{2} \ddot{\tilde{R}} \\
& \approx \varrho_{1} 4 \pi n R_{\mathrm{n}}^{2} \ddot{\tilde{R}}
\end{aligned}
$$

Die Linearisierung im Radius (3.18) führt auf die linearisierte WijngaardenGleichung:

$$
\frac{1}{C_{1}^{2}} \frac{\partial^{2} \tilde{p}}{\partial t^{2}}-\Delta \tilde{p}=\varrho_{1} 4 \pi n R_{\mathrm{n}}^{2} \ddot{\tilde{R}}
$$

Um die noch unbekannte Größe $\ddot{\widetilde{R}}$ zu beschreiben, wird die Lösung der linearisierten Keller-Miksis-Gleichung (2.13) herangezogen:

$$
\begin{gathered}
\tilde{R}(t)=\frac{\tilde{p}(t)}{\varrho_{\mathrm{l}} R_{\mathrm{n}}}\left(\frac{1}{\omega^{2}-\omega_{\text {res }}^{2}-\imath \omega \delta}\right) \\
\delta=\frac{4 \mu}{\varrho_{\mathrm{l}} R_{\mathrm{n}}^{2}}+\frac{\omega_{\text {res }}^{2} R_{\mathrm{n}}}{C_{\mathrm{l}}}, \quad \omega_{\text {res }}^{2}=\frac{1}{\varrho_{\mathrm{l}} R_{\mathrm{n}}^{2}}\left(3 \eta\left(p_{\text {stat }}+\frac{2 \sigma}{R_{\mathrm{n}}}\right)-\frac{2 \sigma}{R_{\mathrm{n}}}\right)
\end{gathered}
$$

Diese Linearisierung eingesetzt in (3.19) ergibt:

$$
\begin{aligned}
& \frac{1}{C_{1}^{2}} \frac{\partial^{2} \tilde{p}}{\partial t^{2}}-\Delta \tilde{p}=\frac{\varrho_{1} 4 \pi n R_{\mathrm{n}}^{2}}{\varrho_{1} R_{\mathrm{n}}} \frac{\partial^{2} \tilde{p}}{\partial t^{2}}\left(\frac{1}{\omega^{2}-\omega_{\text {res }}^{2}-\imath \omega \delta}\right) \\
\Rightarrow & \left(\frac{1}{C_{1}^{2}}-4 \pi n(\vec{x}) R_{\mathrm{n}} \frac{1}{\omega^{2}-\omega_{\text {res }}^{2}-\imath \omega \delta}\right) \frac{\partial^{2} \tilde{p}}{\partial t^{2}}-\Delta \tilde{p}=0
\end{aligned}
$$

Zur Betrachtung der Gültigkeit der Linearisierung wird das System aus (3.20) in Abbildung 3.8 der nichtlinearen Wijngaarden-Wellengleichung (3.14), wiederum für den eindimensionalen Fall, gegenübergestellt. Die Parameter entsprechen denen von Abbildung 3.7 (Mitte). Die zu sehende Übereinstimmung in der raumzeitlichen Entwicklung des Stehwellenfeldes bis etwa $t=150 \mu$ s rechtfertigt für die bis dahin auftretenden Druckamplituden $\left(p_{\mathrm{A}} \leq 30 \mathrm{kPa}\right)$ den Linearisierungsansatz. Bei den später auftretenden Amplituden bis etwa $150 \mathrm{kPa}$ wird die Stehwellenstruktur grob vom linearen Modell wiedergegeben, das Aufsteilen der Wellenfronten und die Druckspitzen im Blasenkollaps werden aber nicht erfasst.

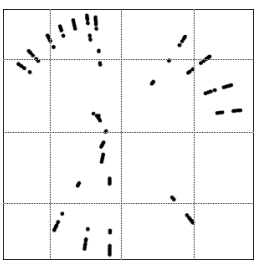



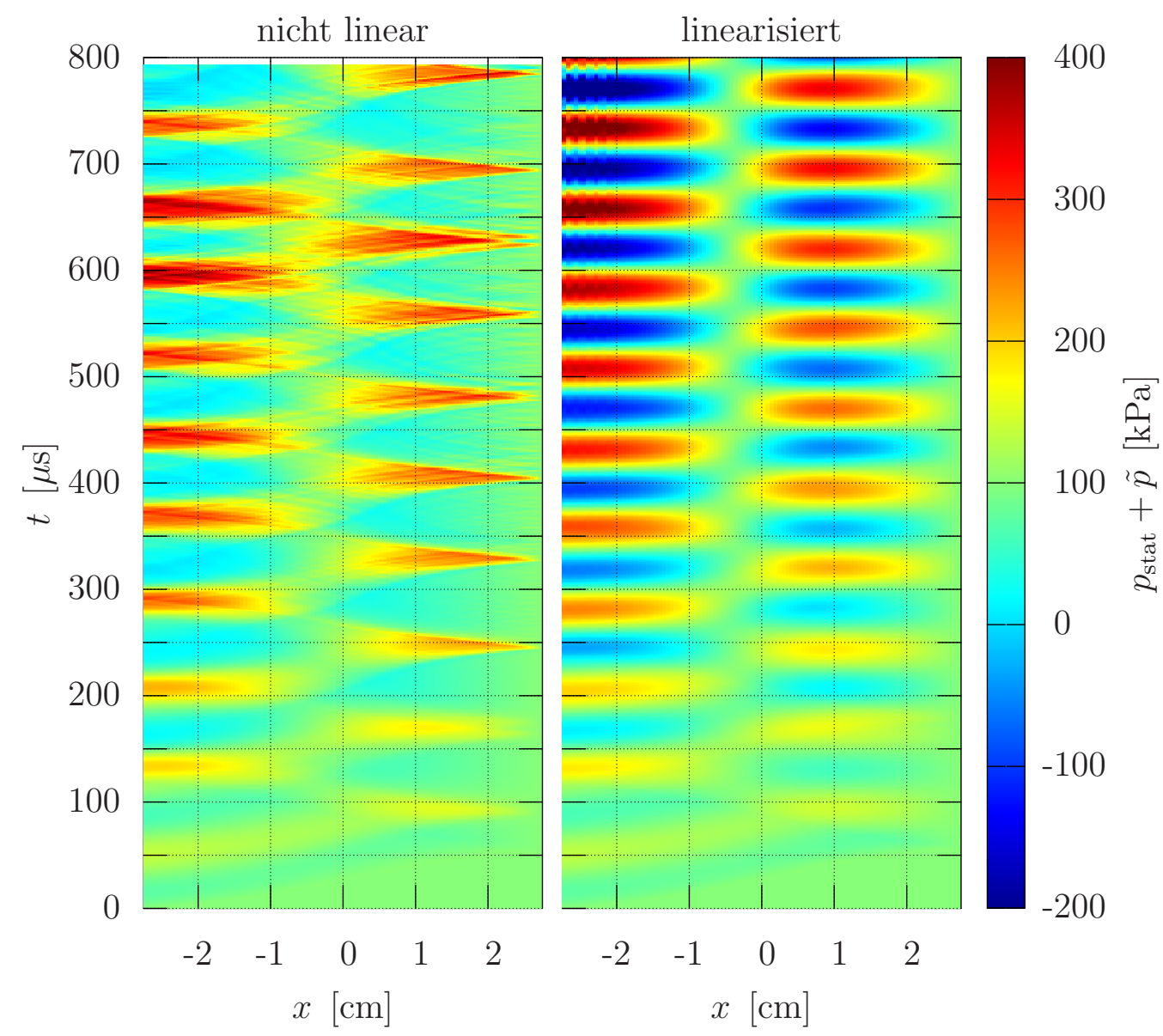

Abbildung 3.8: Gegenüberstellung der nichtlinearen und linearen Lösung der Wellengleichung im Eindimensionalen mit der in Abbildung 3.7 (Mitte) gezeigten Blasenzahlverteilung. links: nichtlineare Lösung der Wijngaarden-Gleichung (3.14) rechts: lineare Lösung der linearisierten Wijngaarden-Gleichung $(3.20) ; \nu=13.47 \mathrm{kHz}, p_{\text {stat }}=100 \mathrm{kPa}, p_{\mathrm{A}}=$ $30 \mathrm{kPa}, R_{\mathrm{n}}=5 \mu \mathrm{m}, N_{\mathrm{yz}}=10^{10}$ Blasen $/ \mathrm{m}^{2}, \Delta_{\mathrm{hwb}}=2 \mathrm{~cm}$.

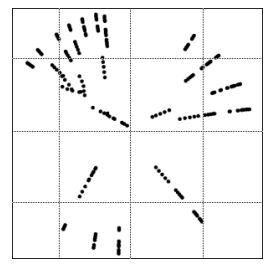


Lösungen der linearisierten Wellengleichung (3.20) im Eindimensionalen sind in Abbildung 3.9 gezeigt. Für kleine Schalldruckamplituden $\left(p_{\mathrm{A}}=50 \mathrm{kPa}\right)$ entspricht die lineare Näherung der in Abbildung 3.7 (Mitte) gezeigten nichtlinearen Lösung. Im Fall stärkerer Schalldruckamplituden $\left(p_{\mathrm{A}}=150 \mathrm{kPa}\right)$ skaliert die Lösung des linearen Systems mit der Anregung.
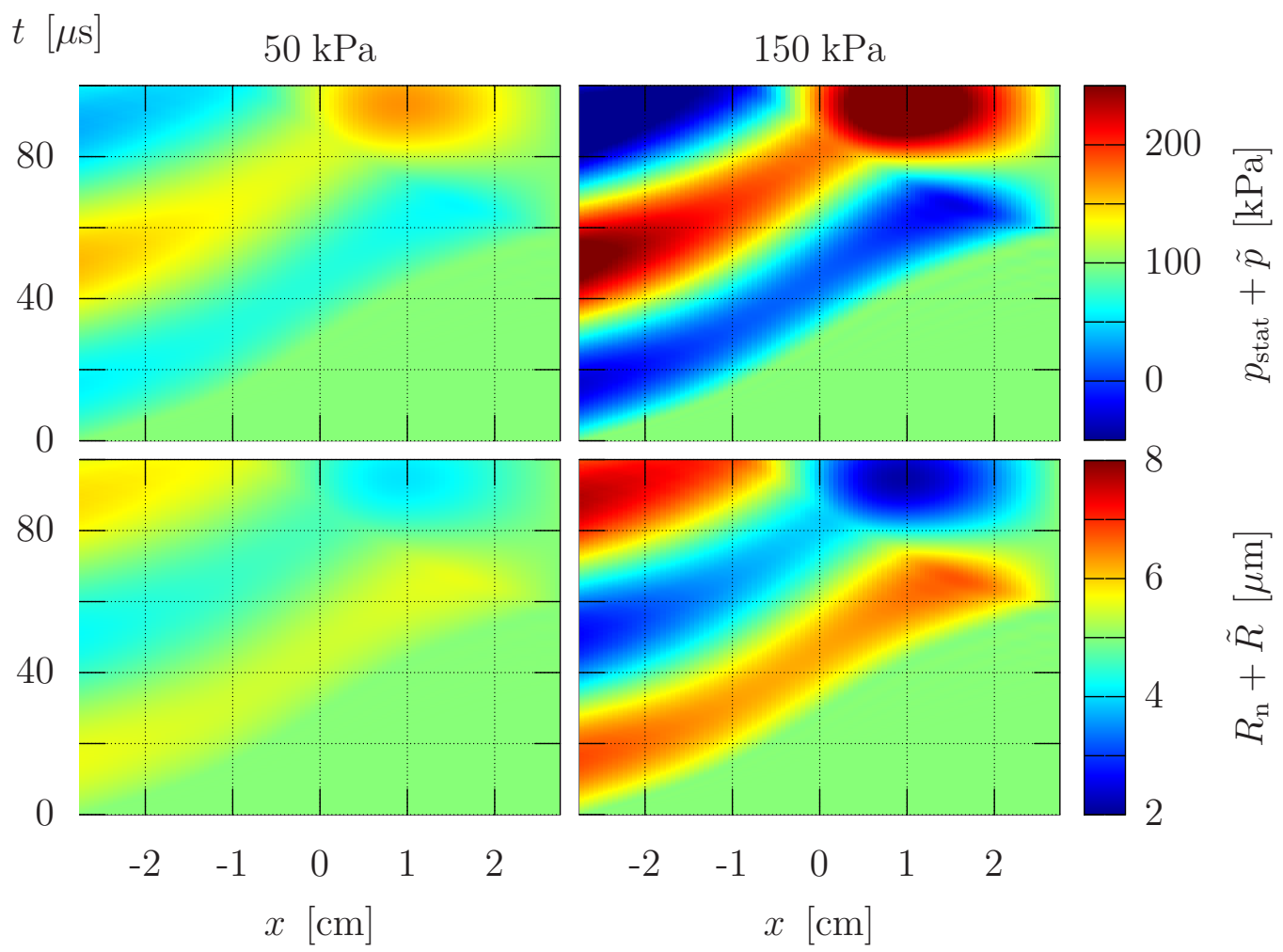

\begin{abstract}
Abbildung 3.9: Die oberen Abbildungen zeigen Lösungen der linearisierten Wijngaarden-Wellengleichung (3.20) zu unterschiedlichen Druckamplituden mit gaußförmiger Blasenzahldichteverteilung. Die unteren Auftragungen zeigen die entsprechenden linearisierten Radienverläufe nach $(2.13) ; \nu=13.47 \mathrm{kHz}, R_{\mathrm{n}}=5 \mu \mathrm{m}, p_{\text {stat }}=100 \mathrm{kPa}, p_{\mathrm{A}}=50,150 \mathrm{kPa}$, $\Delta_{\text {hwb }}=2 \mathrm{~cm}, N_{\text {yz }}=10^{10}$ Blasen $/ \mathrm{m}^{2}$.
\end{abstract}

Deutliche Abweichungen des linearisierten Keller-Miksis-Modells (2.13) von dem realistischen nichtlinearen Verhalten ergeben sich spätestens mit dem Überschreiten der in Abschnitt 2.2 diskutierten Blake-Schwelle. Durch die linearisierte Betrachtung bleiben die nichtlinearen Effekte aus, die das starke Aufschwingen der Blase bewirken. Außerdem ist mit der Linearisierung zu beachten, dass die harmonisch getriebenen Blasen symmetrisch um ihre Ruhelage schwingen, das heißt, erreicht ihre Auslenkung im Unterdruckbereich gerade die Größe ihres Ruhera-

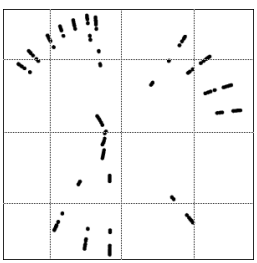


dius $\left(\tilde{R}=R_{\mathrm{n}}\right)$, so wird ihr Radius im Druckmaximum gerade Null $\left(\tilde{R}=-R_{\mathrm{n}}\right)$. Eine $5 \mu \mathrm{m}$ Blase würde etwa für Druckwerte $p_{\mathrm{A}}>350 \mathrm{kPa}$ negative Radien annehmen.

\subsubsection{Lösungen im Dreidimensionalen}

Im Dreidimensionalen wird die linearisierte Wellengleichung (3.20) im eingeschwungenen, zeitharmonischen Zustand betrachtet. Das heißt, alle variablen Größen werden als sinusförmig in der Zeit variierend angenommen. Betrachtet werden dabei die ausgebildeten Stehwellenfelder bzw. Eigenmoden des Systems. Die linearisierte Wellengleichung (3.20) nimmt die Form einer HelmholtzGleichung an, sofern eine zeitharmonische Lösung (3.2) vorausgesetzt wird:

$$
\tilde{p}=p_{\mathrm{A}}(x) \sin (\omega t) \quad \Rightarrow \quad \ddot{\tilde{p}}=-\omega^{2} \tilde{p}
$$

In die linearisierte Wellengleichung (3.20) eingesetzt liefert dieser Ansatz die modifizierte Helmholtz-Gleichung mit erweitertem Schallgeschwindigkeitsterm:

$$
\left(-\frac{\omega^{2}}{C_{1}^{2}}+4 \pi n(\vec{x}) R_{\mathrm{n}} \frac{\omega^{2}}{\omega^{2}-\omega_{\text {res }}^{2}-\imath \omega \delta}\right) p_{\mathrm{A}}-\Delta p_{\mathrm{A}}=0
$$

Diese Gleichung liefert eine harmonisch in der Zeit oszillierende ortsabhängige Lösung.

Die durch die Blasenzahldichte modifizierte Schallgeschwindigkeit in dem Gemisch lautet:

$$
C_{\mathrm{m}}=\left(\frac{1}{C_{1}^{2}}-\frac{4 \pi n(\vec{x}) R_{\mathrm{n}}}{\omega^{2}-\omega_{\mathrm{res}}^{2}-\imath \omega \delta}\right)^{-1 / 2}
$$

Im Grenzfall eines blasenfreien Systems $(n=0, \forall \vec{x})$ ergibt sich die ungestörte Schallgeschwindigkeit im Fluid $C_{\mathrm{m}}=C_{\mathrm{l}}$. Ansonsten wird die Blasenzahldichte auf der Zeitskala, auf der das Schallfeld berechnet wird, als zeitlich konstant angenommen.

Hier wird zunächst ein einheitlicher Ruheradius angesetzt. Ansonsten wäre eine Summation bzw. Integration des rechten Termes in der Klammer in (3.22) über alle beteiligten Blasengrößen und ihre zugehörigen Dichten notwendig, worauf hier der einfachheithalber verzichtet wird.

Die in den Abbildungen 3.2 und 3.3 gezeigte dreidimensionale Geometrie der Küvette mit angebrachtem Piezoring wurde über MATLAB [66] in dem Programmpaket FEMLAB [57] dargestellt.

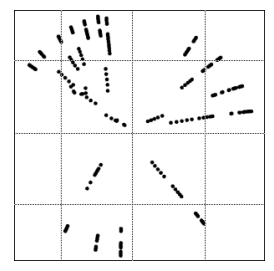


Das System (3.21) wird angetrieben durch harmonische Auslenkungen der Piezoringfläche:

$$
x=d \sin (\omega t) \quad \Rightarrow \quad \ddot{x}=-d \omega^{2} \sin (\omega t)
$$

Alle anderen Randbedingungen sind als schallweiche Abschlüsse definiert:

$$
p_{\text {Rand }}=0
$$

Als Testverteilung von Blasen und für die Darstellung des Effekts der modifizierten Schallgeschwindigkeit werden wiederum gaußförmige Blasendichteverteilungen radialsymmetrisch um das geometrische Zentrum eingesetzt. Die Auftragungen in Abbildung 3.10 zeigen die zeitharmonischen Lösungen der modifizierten Helmholtz-Gleichung (3.21). Die sich einstellende Schallgeschwindigkeitsverteilung im Blasen-Fluid-Gemisch ist in Abbildung 3.10 (links) aufgetragen. Abbildung 3.10 (rechts) zeigt Lösungen der modifizierten Helmholtz-Gleichung (3.21) für die verschiedenen gaußförmigen Kugelverteilungen von Blasen im Zentrum der Küvette.

Um eine Eigenfrequenzanalyse der modifizierten Helmholtz-Gleichung (3.21) mit der frequenzabhängigen Schallgeschwindigkeit (3.22) durchführen zu können wird diese Abhängigkeit unter Einsatz der linearen Blasen-Resonanzfrequenz (2.12b) abgeschätzt. Bei Vernachlässigung der Oberflächenspannung gilt für die lineare Resonanzfrequenz die bekannte Minnaert'sche Form (2.7):

$$
\omega_{\mathrm{res}} R_{\mathrm{n}}=3 \cdot 2 \pi
$$

Unter Verwendung dieses Zusammenhangs schreibt sich die Abschätzung der linearen Resonanzfrequenz $\omega_{\text {res }}$ gegen die treibende Frequenz $\omega$ als:

$$
\omega_{\text {res }} \gg \omega \text { falls } 3 / R_{\mathrm{n}} \gg \nu
$$

Diese Abschätzung ist gültig für die typischen Größen von $R_{\mathrm{n}} \approx 5 \mu \mathrm{m}$ und $\nu \approx 20 \mathrm{kHz}$. Die von der treibenden Frequenz unabhängige Schallgeschwindigkeit lautet dann:

$$
\widehat{C}_{\mathrm{m}} \approx\left(\frac{1}{C_{1}^{2}}+\frac{4 \pi n(\vec{x}) R_{\mathrm{n}}}{\omega_{\mathrm{res}}^{2}}\right)^{-1 / 2}
$$

Eine erhöhte Blasendichte in dem Gemisch führt zu lokalen Änderungen der Schallgeschwindigkeit womit es zu der in Abbildung 3.11 gezeigte Resonanzverschiebung des Systems kommt. Wird dabei die treibende Frequenz nicht

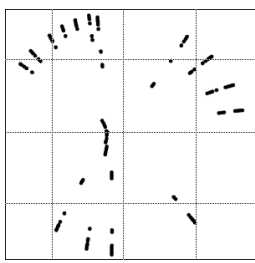



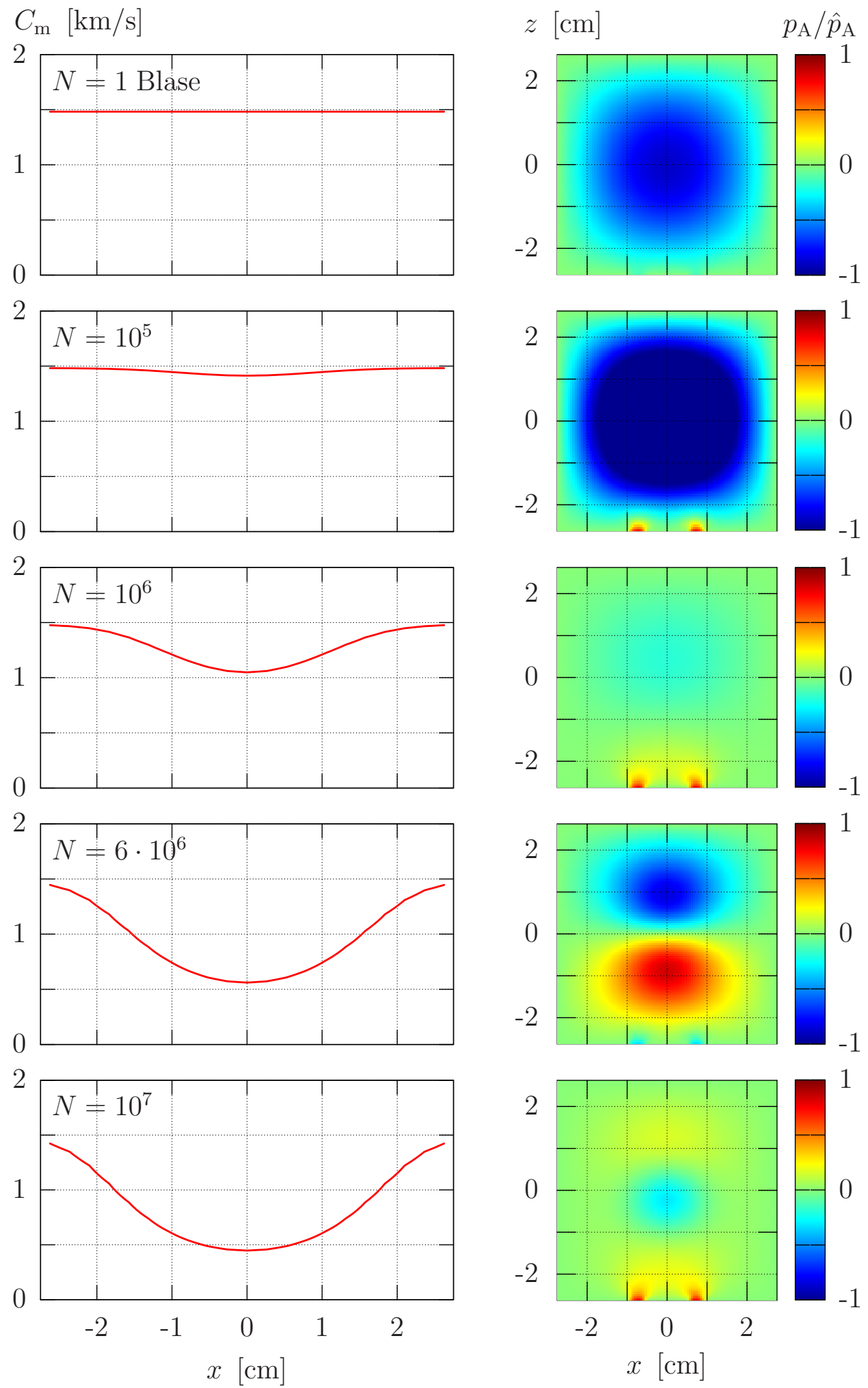

Abbildung 3.10: Verlauf der Schallgeschwindigkeit im Gemisch (links) mit den zugehörigen qualitativ aufgetragenen Schallfeldmoden (rechts, radialsymmetrisch um die $x$-Achse) als Lösungen der modifizierten Helmholtz-Gleichung (3.21); $\nu=23.647 \mathrm{kHz}, C_{\mathrm{l}}=1482 \mathrm{~m} / \mathrm{s}, R_{\mathrm{n}}=5 \mu \mathrm{m}$, $N=1,10^{5}, 10^{6}, 6 \cdot 10^{6}, 10^{7}$ Blasen, $\Delta_{\text {hwb }}=2 \mathrm{~cm}$.

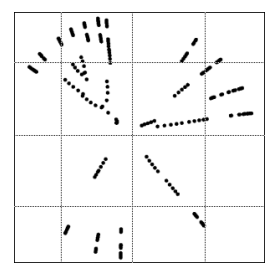




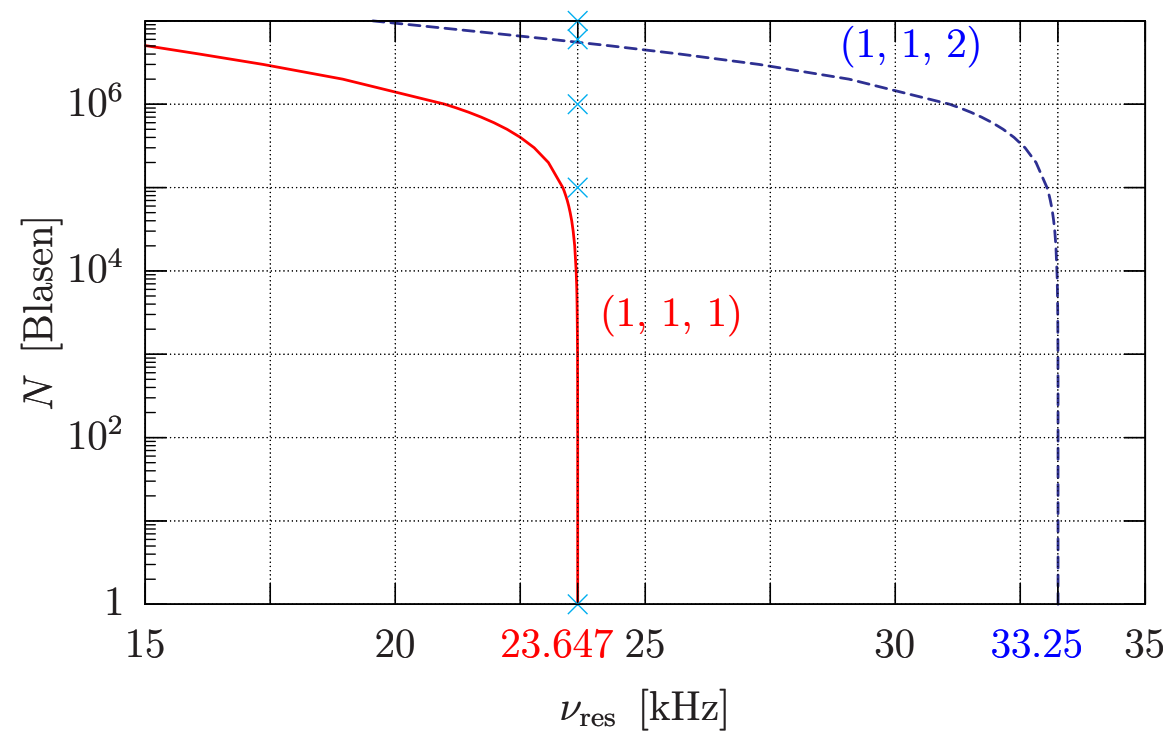

Abbildung 3.11: Die Resonanzverschiebung in der $(1,1,1)$ - und $(1,1,2)$-Mode in Abhängigkeit von der Gesamtblasenzahl bei einer gaußförmigen Blasenverteilung nach (3.15) ergibt sich aus den Eigenfrequenzen der modifizierten Helmholtz-Gleichung (3.21) mit Verwendung der nach Minnaert abgeschätzten Schallgeschwindigkeit (3.23); Die mit Kreuzen markierten Parameterwerte entsprechen den in Abbildung 3.10 betrachteten Moden des Systems; $\Delta_{\text {hwb }}=2 \mathrm{~cm}, D=0, \nu=23.647 \mathrm{kHz}$, $C_{\mathrm{l}}=1482 \mathrm{~m} / \mathrm{s}, R_{\mathrm{n}}=5 \mu \mathrm{m}$.

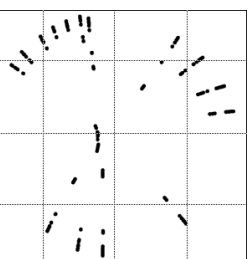


nachgeregelt, so kommt es in dem gezeigten Parameterbereich zu einem Modenübergang von der $(1,1,1)$ - auf die $(1,1,2)$-Mode.

Die Verschiebung der Resonanzfrequenz wird wesentlich durch die Blasenzahldichte bestimmt. Die Betrachtung der Abhängigkeit von der Dämpfung in Abbildung 3.6 zeigt keine signifikante Verschiebung der Resonanz in dem vorliegenden Parameterbereich.

Werden die Resonanzkurven aus Abbildung 3.5 (Mitte) dem Verlauf der Resonanzverschiebung in Auftragung 3.11 folgend über die ResonanzfrequenzBlasenzahldichte-Ebene verschoben, so ergibt sich ein Gebirge, das die Schalldruckamplitude anzeigt. Der Schnitt durch dies Gebirge in Abbildung $3.12 \mathrm{zu}$ fester treibender Frequenz zeigt den Druckabfall mit zunehmender Gesamtblasenzahl an, bedingt durch die Resonanzverschiebung.

Die „Unebenheiten“ in dem in Abbildung 3.12 gezeigten Druckverlauf gehen auf ein in der in Abbildung 3.11 gezeigten Resonanzverschiebung nicht aufgelöstes „Zappeln“ zurück. Dies numerische Artefakt rührt daher, dass die notwendige Blasenverteilung zur Lösung der modifizierten Helmholtz-Gleichung (3.21) auf einem diskretisierenden Gitter angegeben wird.

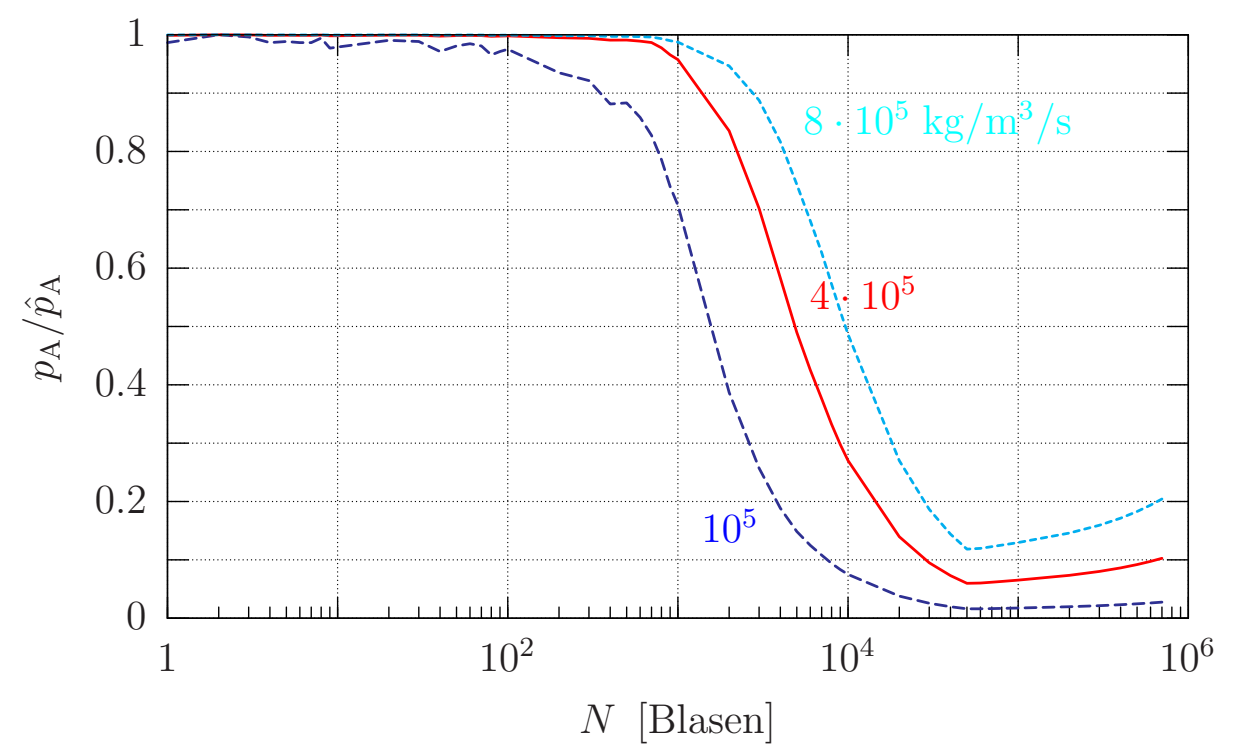

Abbildung 3.12: Druckverlauf in Abhängigkeit von der Gesamtblasenanzahl unter Einbeziehung der Dämpfung. Der Zusammenhang von Schalldruckamplitude und Blasenanzahl ergibt sich durch die Betrachtung der Resonanzkurven aus Abbildung 3.5 (Mitte), deren Maximum entsprechend der Resonanzverschiebung aus Abbildung 3.11 verschoben wird; $\nu=23.647 \mathrm{kHz}, \Delta_{\text {hwb }}=2 \mathrm{~cm}, D=10^{5}, 4 \cdot 10^{5}, 8 \cdot 10^{5} \mathrm{~kg} / \mathrm{m}^{3} / \mathrm{s}$.

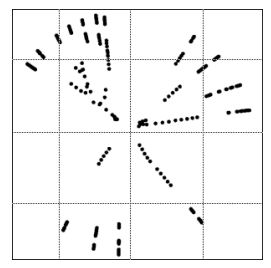


Die Dämpfung in dem Resonator wird berücksichtigt, indem die bereits im Schallgeschwindigkeitsterm modifizierte, lineare Wijngaarden-Wellengleichung (3.20) erweitert wird um den nach (3.4) bekannten Dämpfungsterm:

$$
\underbrace{\left(\frac{1}{C_{1}^{2}}-\frac{4 \pi n(\vec{x}) R_{\mathrm{n}}}{\omega^{2}-\omega_{\text {res }}^{2}-\imath \omega \delta}\right)}_{=1 / C_{\mathrm{m}}^{2}} \frac{\partial^{2} \tilde{p}}{\partial t^{2}}-\Delta \tilde{p}+\frac{D}{\varrho_{1} C_{1}^{2}} \frac{\partial \tilde{p}}{\partial t}=0
$$

Die blasenbedingte Dämpfung ist bereits in dem komplexen Anteil der unter Anwesenheit von Blasen modifizierten Schallgeschwindigkeit enthalten:

$$
\frac{1}{C_{\mathrm{m}}^{2}}=\frac{1}{C_{1}^{2}}-\frac{4 \pi n(\vec{x}) R_{\mathrm{n}}\left(\omega^{2}-\omega_{\mathrm{res}}^{2}\right)+\imath 4 \pi n(\vec{x}) R_{\mathrm{n}} \omega \delta}{\left(\omega^{2}-\omega_{\mathrm{res}}^{2}\right)^{2}+\omega^{2} \delta^{2}}
$$

Um diese linearisierte gedämpfte Wijngaarden-Wellengleichung in die Form einer zeitunabhängigen Helmholtz-Gleichung zu bringen wird ein komplexer Lösungsansatz gewählt:

$$
\tilde{p}=p_{\mathrm{A}} \mathrm{e}^{\imath \omega t}
$$

Eingesetzt in (3.24) ergibt die modifizierte gedämpfte HelmholtzGleichung:

$$
\widehat{k}^{2} p_{\mathrm{A}}+\Delta p_{\mathrm{A}}=0
$$

Die modifizierte komplexe Wellenzahl schreibt sich als:

$$
\begin{aligned}
\widehat{k}^{2} & =\frac{\omega^{2}}{C_{\mathrm{m}}^{2}}-\imath \frac{\omega D}{C_{1}^{2} \varrho_{\mathrm{l}}} \\
& =\frac{\omega^{2}}{C_{1}^{2}}-\frac{4 \pi n(\vec{x}) R_{\mathrm{n}}\left(\omega^{2}-\omega_{\mathrm{res}}^{2}\right) \omega^{2}}{\left(\omega^{2}-\omega_{\mathrm{res}}^{2}\right)^{2}+\omega^{2} \delta^{2}}-\imath\left(\frac{4 \pi n(\vec{x}) R_{\mathrm{n}} \omega^{3} \delta}{\left(\omega^{2}-\omega_{\mathrm{res}}^{2}\right)^{2}+\omega^{2} \delta^{2}}-\frac{\omega D}{C_{1}^{2} \varrho_{\mathrm{l}}}\right)
\end{aligned}
$$

Im Grenzfall des ungedämpften Systems $(D=0)$ ist diese Darstellung äquivalent zu der modifizierten Helmholtz-Gleichung (3.21).

\subsubsection{Einzelblase in der Küvette}

Von Krefting [19] wurde ein deutlicher Einfluss einzelner Blasen auf den Resonator festgestellt. Die Messungen werden im linearen Dynamikbereich $\left(\hat{p}_{\mathrm{A}}<\right.$ $50 \mathrm{kPa}$ ) für Blasen von etwa $50 \mu \mathrm{m}$ Ruheradius durchgeführt. Aufgrund der relativ langen Messzeiten zur Aufnahme einer Resonanzkurve, ist ein diffusionsbedingtes Anwachsen der Blase zu erwarten. Für die Messungen werden einzelne

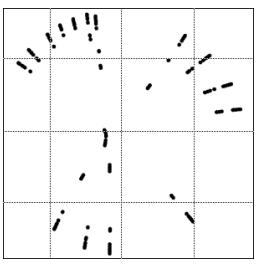


Blasen in die quaderförmige Hellma-Küvette injiziert, in der sie zunächst in einer konstanten Schalldruckmode, nahe der $(1,1,1)$-Mode gehalten werden.

Die Resonanzkurve des Systems aus wasserbefüllter Küvette mit Piezoring und Blase wird aufgenommen, indem die sich mit der treibenden Frequenz ändernden Wandauslenkungen gemessen werden. Schon bei der in Abbildung 3.13 gezeigten schwachen Anregung $\left(\hat{p}_{\mathrm{A}} \approx 15.8 \mathrm{kPa}\right)$ ist gegenüber dem blasenfreien System eine Verschiebung um etwa $10 \mathrm{~Hz}$ deutlich zu erkennen.

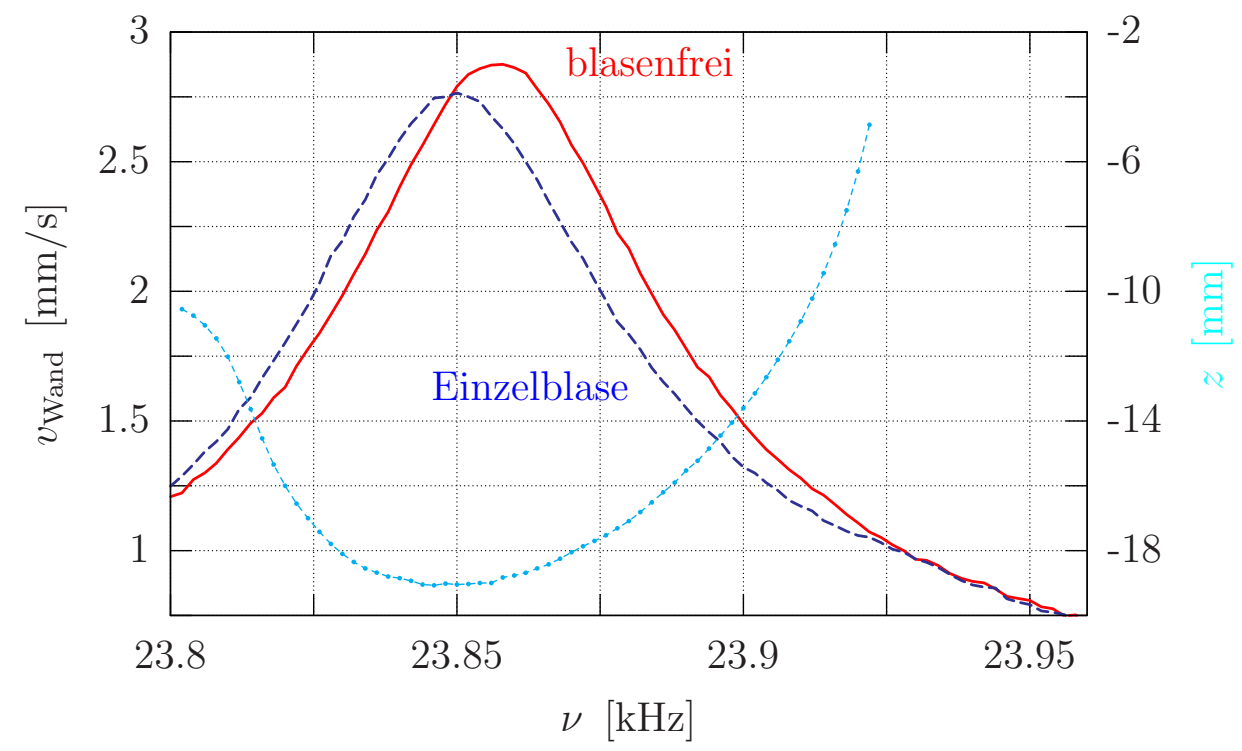

Abbildung 3.13: Vergleich der Resonanzkurve des blasenfreien Systems mit der verschobenen Resonanz, beim Auftreten einer Blase bei schwacher Anregung; die gepunktete Linie gibt die Blasenposition entlang der vertikalen $z$-Achse an ( $z=0$ entspricht der Wasseroberfläche); $R_{\mathrm{n}} \approx 50 \mu \mathrm{m}$, $\hat{p}_{\mathrm{A}} \approx 15.8 \mathrm{kPa}$. (Messung von Krefting [19])

Neben den Resonanzkurven ist in Abbildung 3.13 die vertikale Position der Blase aufgetragen. Entlang der gepunkteten Linie befindet sich die Blase an ihrer Gleichgewichtsposition aus Auftrieb und der sie entlang des Druckgradienten zum maximalen Schalldruck ziehenden Kraft. Entlang der fallenden Flanke der Resonanzkurve steigt die Blase auf; die Wasseroberfläche liegt bei $z=0$. Bei einer Frequenz knapp oberhalb $23.92 \mathrm{kHz}$ tritt die Blase aus der Küvette aus, und die Resonanzkurve deckt sich ab diesem Punkt mit der Kurve des zuvor ausgemessenen blasenfreien Systems.

Die Betrachtung bei einer hohen Anregungsamplitude $\left(\hat{p}_{\mathrm{A}} \approx 44.6 \mathrm{kPa}\right)$ im Bereich linearer Blasendynamik ist in Abbildung 3.14 gezeigt. Die injizierte Blase

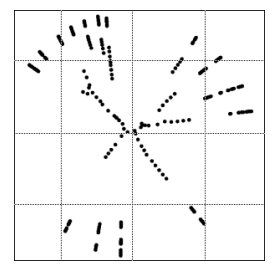


hält sich hier zunächst nahe der Oberfläche $(z=0)$ und führt erst mit dem Absinken in Richtung des geometrischen Zentrums der Küvette zu einer Abweichung der Resonanzkurve von der des blasenfreien Systems (bei $\nu \approx 23.79 \mathrm{kHz}$ ). Mit Anwesenheit der Blase fällt die auftretende Resonanzspitze $\nu \approx 23.865 \mathrm{kHz}$ etwa um den Faktor zwei schwächer aus. Im Bereich der Resonanz befindet sich die Blase gerade an ihrer dem Zentrum der Küvette nächstgelegenen Position $z \approx-19 \mathrm{~mm}$.

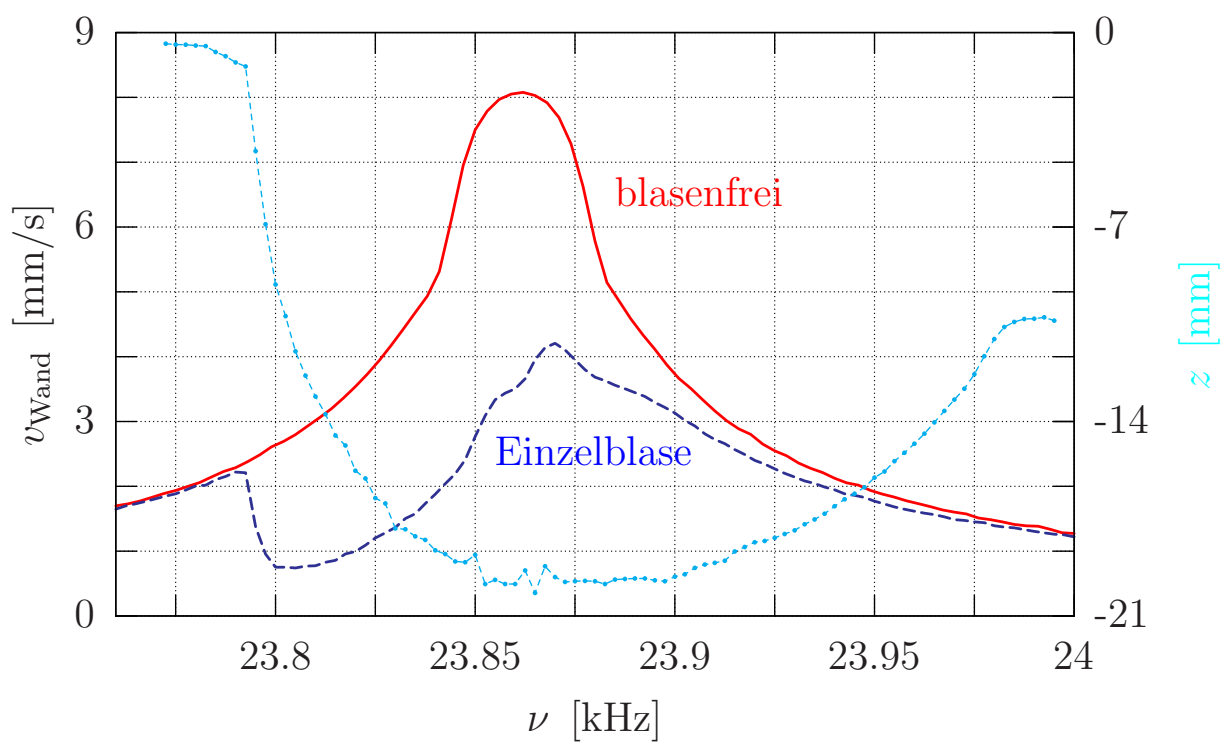

Abbildung 3.14: Vergleich der Resonanzkurve des blasenfreien Systems mit der verschobenen Resonanz, beim Auftreten einer Blase bei hoher Anregungsamplitude noch im Bereich einer angenommenen linearen Blasendynamik; die gepunktete Linie gibt die Blasenposition entlang der vertikalen $z$-Achse an ( $z=0$ entspricht der Wasseroberfläche); $R_{\mathrm{n}} \approx 50 \mu \mathrm{m}$, $\hat{p}_{\mathrm{A}} \approx 44.6 \mathrm{kPa}$. (Messung von Krefting [19])

In der numerischen Betrachtung wird der Übergang von der zuvor in Abbildung 3.11 betrachteten Resonanzverschiebung in Vielblasensystemen hin zu einer realistischen Abschätzung weniger, bis einzelner Blasen ausgeführt. Dabei muss mit der Gesamtblasenzahl auch die Halbwertsbreite der angenommenen gaußförmigen Blasenverteilung (3.15) den Ruheradien angepasst werden. Im Grenzfall einer Einzelblase wird angenommen:

$$
\Delta_{\mathrm{hwb}} \approx 2 \cdot R_{\mathrm{n}}
$$

Die in Abbildung 3.15 gezeigte Ausschnittsvergrößerung von Abbildung 3.11 zeigt die Resonanzverschiebung bei niedrigen Blasenzahlen. Wird die Gesamtblasenzahl mit einer niedrigeren Halbwertsbreite auf einem engeren Raumbereich um

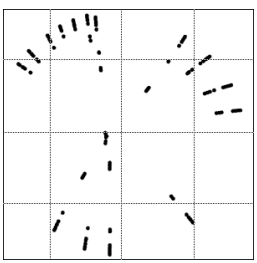


das Zentrum konzentriert, so verstärkt sich die Verschiebung der Resonanzfrequenz. Für $N=1000$ Blasen kommt es dabei mit der Umgruppierung von $\Delta_{\text {hwb }}=2 \mathrm{~cm}$ auf $\Delta_{\text {hwb }}=5 \mathrm{~mm}$ zu einer Resonanzverschiebung von etwa $10 \mathrm{~Hz}$.

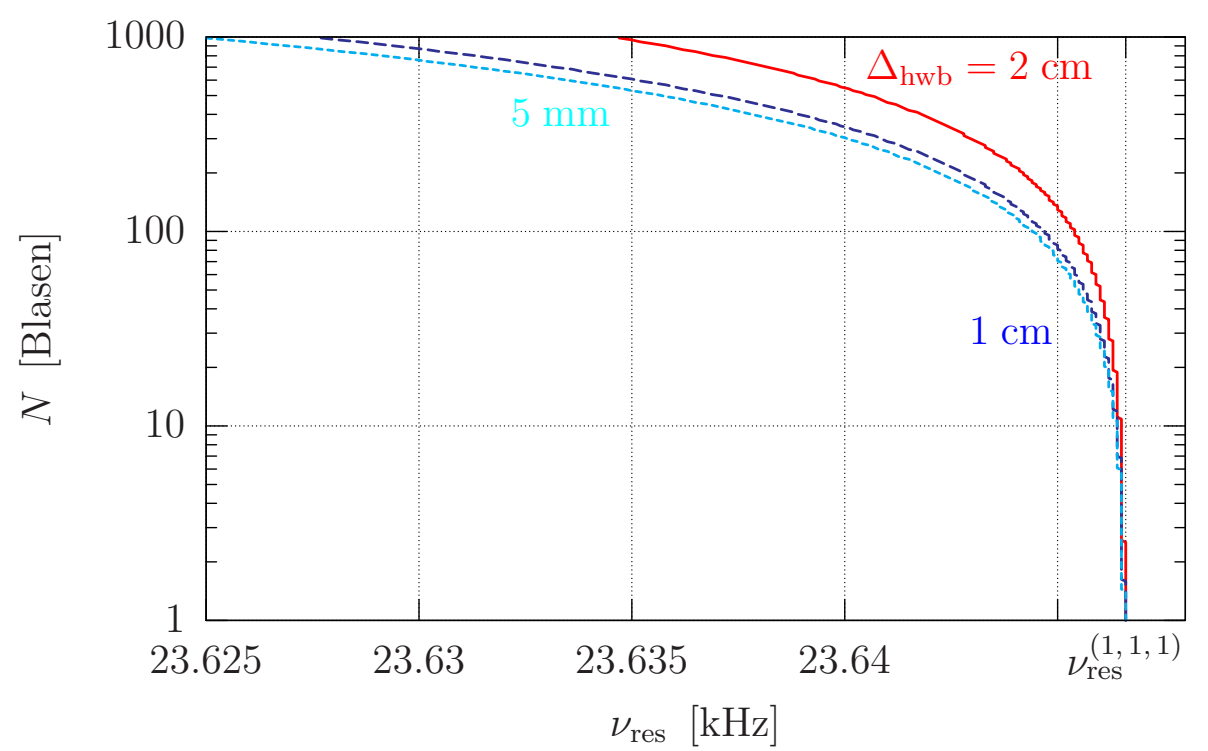

Abbildung 3.15: Betrachtung der Resonanzverschiebung aus der Eigenfrequenzanalyse der modifizierten Helmholtz-Gleichung (3.21) für kleine Gesamtblasenzahlen bei abnehmender Halbwertsbreite der gaußförmigen Blasenverteilung. Getrieben wird das System mit der Resonanzfrequenz, die sich im blasenfreien Fall für die $(1,1,1)$-Mode einstellt. $N=1 \ldots 1000$ Blasen, $\Delta_{\text {hwb }}=2,1,0.5 \mathrm{~cm}, \nu=\nu_{\text {res }}^{(1,1,1)}=23.647 \mathrm{kHz}$, $R_{\mathrm{n}}=5 \mu \mathrm{m}$.

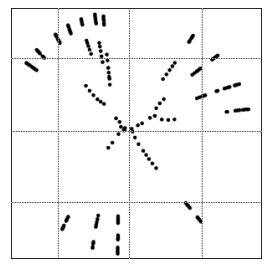




\subsection{Laufendes Wellenfeld}

Die fortlaufend über die Piezokeramik und die Wand in den Resonator eingekoppelten laufenden Wellen überlagern sich mit bereits reflektierten Wellenzügen, wodurch sich ein geometrieabhängiges Stehwellenfeld ausbildet. Mit Änderung der Impedanz, etwa durch ständig fluktuierende Blasenverteilungen, können auf kurzen Zeitskalen laufende Wellenanteile auftreten. Die Ausbildung der Stehwellenmode findet jedoch wie aus Abbildung 3.8 ersichtlich innerhalb weniger $100 \mu \mathrm{s}$ statt, demnach deutlich schneller, als normalerweise eine Blasenumgruppierung erfolgt. Typische Blasenstrukturen erweisen sich über den Zeitraum mehrerer Sekunden als stabil. Somit kann im Allgemeinen von stehenden Wellenfeldern in den betrachteten Resonatoren ausgegangen werden, wie sie abhängig von der Gesamtblasenzahl beispielsweise in der Abbildung 3.10 gezeigt sind.

Dagegen kann im Fall einer in ein wasserbefülltes Gefäß eingetauchten Sonotrode von einem laufenden Wellenfeld ausgegangen werden. Im Allgemeinen wird über die Sonotrode lokal ein sehr hoher Schalldruck in der Flüssigkeit erzeugt. Der Schall breitet sich ähnlich einer Kugelwelle in das Gefäß aus, wird aber durch Reflexionen nie wieder so konzentriert wie er ursprünglich von der Sonotrodenspitze ausging. Daher gibt es immer einen hohen Anteil, der nur von der Sonotrode wegläuft und grob mit dem Kehrwert des Abstands abfällt. Zudem kann es direkt an und vor der Sonotrode zu starker Blasenbildung kommen. Diese hohe Blasenkonzentration kann „abschirmend“ auf die eingekoppelten Wellen wirken, so dass es zu keinen Reflexionen und Überlagerungen kommt. Die Absorption der Wellen in dem Blasenfeld unterstützt eine fortwährend über die Sonotrode eingekoppelte laufende Welle.

Abbildung 3.16 zeigt die numerisch behandelte Anordnung. Dabei wird eine in ein Wasserbecken getauchte zylinderförmige Sonotrode betrachtet. Um die Ausbreitung laufender Wellen zu ermöglichen, wird das Wasserbecken als unendlich ausgedehnt angenommen, das heißt die Randbedingungen der Seitenwände und des Bodens sind an die Impedanz des Wassers angepasst. Die Wasseroberfläche um die Sonotrode ist schallweich, die seitliche Mantelfläche der Sonotrode schallhart angesetzt. Durch periodische ebene Auslenkungen ${ }^{4}$ der Stirnfläche der Sonotrode wird eine Welle eingekoppelt. In der Rechnung sind keine Blasen enthalten.

Der weiße Bereich in den Auftragungen 3.17 stellt die Sonotrodenspitze dar. Gezeigt ist die normierte ortsabhängige Amplitudenverteilung $p_{\mathrm{A}}$ (links) sowie die

${ }^{4}$ Durch die spezielle Geometrie des Sonotrodenkörpers wird eine Auslenkung der Mantelfläche vermieden und das Anschwingen von Oberflächenmoden auf der Stirnfläche vernachlässigbar klein gehalten.

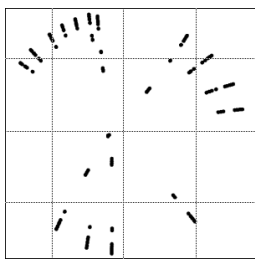




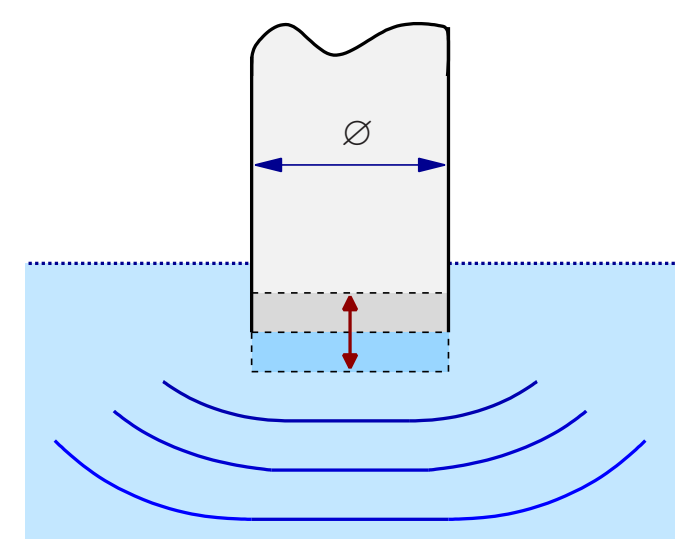

Abbildung 3.16: Abbildung der schwingenden Stirnfläche einer zylindrischen Sonotrode, über die eine laufende Welle in die Flüssigkeit eingekoppelt wird.

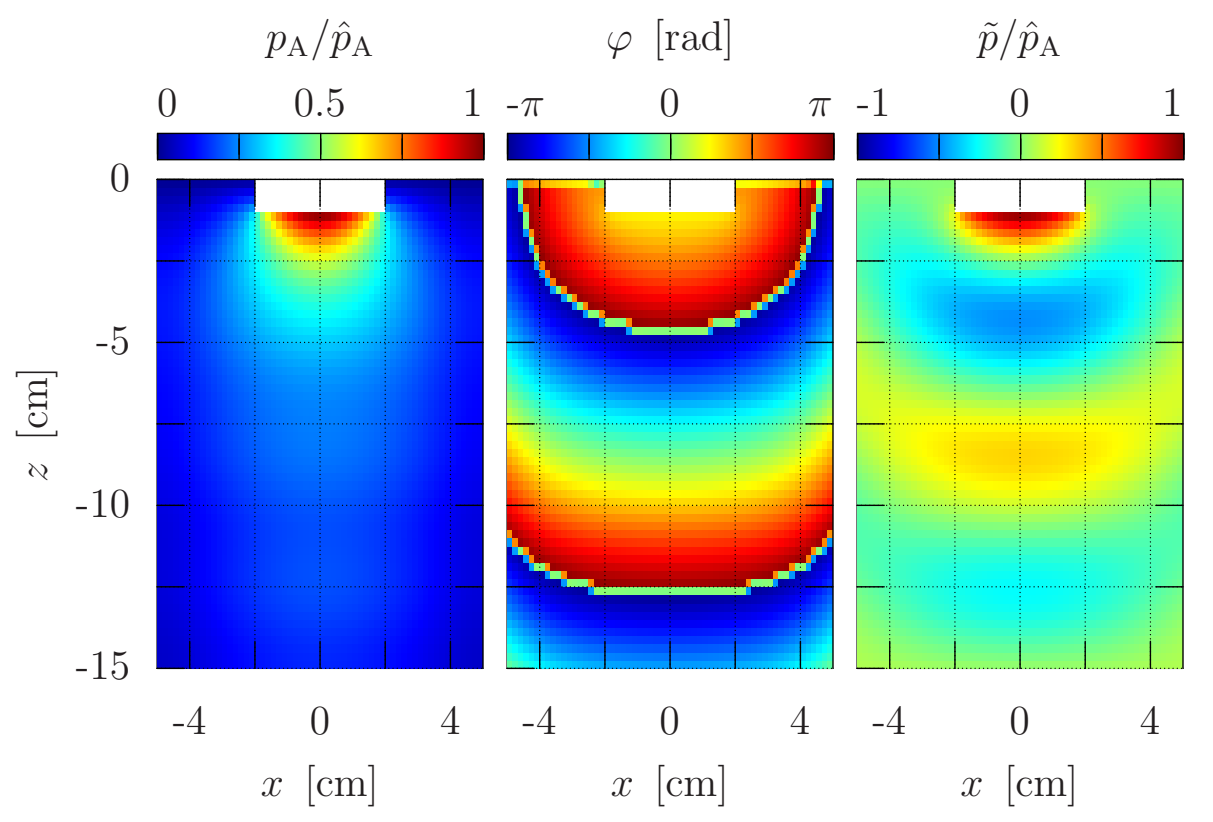

Abbildung 3.17: Zusammenstellung des Amplitudenverlaufs $p_{\mathrm{A}}$ (links) und der Phasenlage $\varphi$ (Mitte) zu der reellen Darstellung der laufenden Welle $\tilde{p}=p_{\mathrm{A}} \cos (\varphi)$ (rechts) unter einer Sonotrodenspitze als Lösung der gedämpften Helmholtz-Gleichung (3.5); Eintauchtiefe $1 \mathrm{~cm}, \varnothing=4 \mathrm{~cm}$, $\nu=20 \mathrm{kHz}, D=3.26 \cdot 10^{5} \mathrm{~kg} / \mathrm{m}^{3} / \mathrm{s}$.

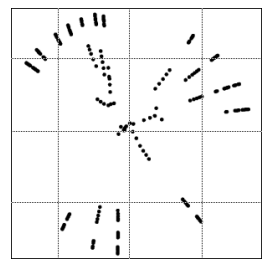


Phasenlage des Schallfeldes $\varphi$ (Mitte). Rechts ist eine Momentaufnahme der laufenden Welle zur Zeit $t=0 \mathrm{~s}$ von $\tilde{p}=p_{\mathrm{A}} \cos (\varphi)$ dargestellt.

Zur besseren qualitativen Betrachtung zeigt Abbildung 3.18 den Verlauf des Schallwechseldrucks als Schnitt durch das rechts in Abbildung 3.17 aufgetragene zylindersymmetrische Schallfeld.

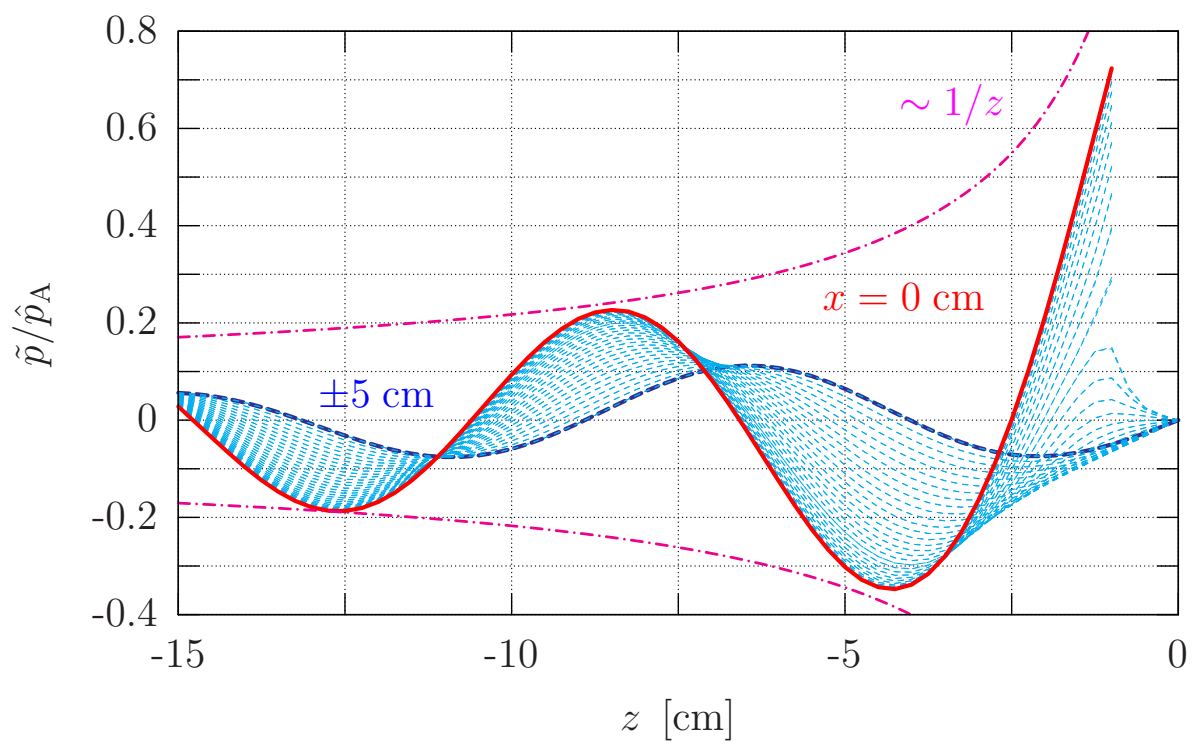

Abbildung 3.18: Darstellung des Druckverlaufs aus Abbildung 3.17 (rechts) in $z$-Richtung zu verschiedenen Abständen von der Symmetrieachse. Die Amplitude fällt in Näherung mit $1 / z \mathrm{ab} ; x=0 \ldots \pm 5 \mathrm{~cm}$.

Die von der Stirnfläche der Sonotrode aus laufende Welle schwächt sich ab, da die Ausbreitung in alle drei Raumrichtungen erfolgt und sich dabei die Wellenfront schnell vergrößert. Im Fall einer ideal punktförmigen Sonotrodenspitze würde die Wellenfront eine mit dem Kehrwert des Abstandes abklingende Kugelwelle bilden. Mit zunehmendem Durchmesser der Sonotrode ähnelt der Zentralbereich um die Symmetrieachse allerdings mehr und mehr einer ebenen Welle.

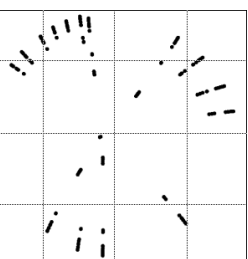




\section{Kapitel 4}

\section{Translationsdynamik}

Dieses Kapitel beschäftigt sich mit den wichtigsten Kräfte, die auf eine Blasen einwirken können, nämlich die virtuelle Trägheitskraft (virtuelle Masse) in Abschnitt 4.1, die Bjerkneskräfte in den Abschnitten 4.2 und 4.3 sowie die Reibungskraft im Abschnitt 4.4. Zusammen bilden sie die Newton'sche Bewegungsgleichung für eine Blase. Die in den vorangegangenen Kapiteln vorgestellten Modelle zur Beschreibung der radialen Blasendynamik (Kapitel 2) sowie der Schallfeldberechnung (Kapitel 3) werden dann in den Gesamtzusammenhang der in Abschnitt 4.5 vorgestellten Partikelmodellierung eingesetzt.

Das Translationsverhalten jeder einzelnen Blase ergibt sich als Lösung der gekoppelten Bewegungsgleichungen aller Blasen im Schallfeld:

$$
\sum_{k} \vec{F}_{k}^{(i)}=\overrightarrow{0}, \quad i=1 \ldots N, \quad k=\mathrm{M}, \mathrm{Bp}, \mathrm{Bs}, \mathrm{D}
$$

\section{$N \quad$ Anzahl der Blasen}

Die Summe aller Kräfte auf eine Blase ist gleich Null. Für die Strukturbildung in Vielblasensystemen sind vier Kräfte von Bedeutung: die virtuelle Masse M, die primäre und sekundäre Bjerkneskraft Bp und Bs sowie die Reibungskraft D über die der Index $k$ läuft.

Durch zeitliche Mittelungen in den Größen der Pulsationsdynamik wird eine Entkopplung der Blasenoszillation und der Translation erreicht. Gerechtfertigt ist dieses Vorgehen durch die unterschiedlichen Zeitskalen auf denen Oszillation und Translation ablaufen:

$$
\tau_{\text {osz }} \ll \tau_{\text {trans }}
$$

Mit der Entkopplung reduziert sich der Rechenaufwand, so dass das resultierende Partikelmodell entsprechend der zur Verfügung stehenden Rechnerleistung für Vielblasensysteme einsetzbar ist. 
Eine weitere Optimierung bezüglich des Zeitaufwandes in der Simulation wird durch die vorherige Tabellierung der gemittelten Größen aus der Pulsationsdynamik erreicht.

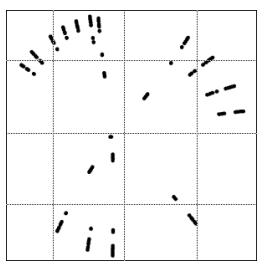




\subsection{Virtuelle Masse}

Die virtuelle Masse ergibt sich aus der durch die Flüssigkeit verursachten Trägheit eines umströmten Körpers. Mit Beschleunigung der Blase nimmt diese Strömung mehr Energie auf. Unter der Annahme der in Abbildung 4.1 skizzierten Potentialströmung $^{1}$ ist der Impuls der Blase erhalten, da sich im Zusammenlauf hinter der Blase die Energie aus der Strömung auf die Blase zurücküberträgt.

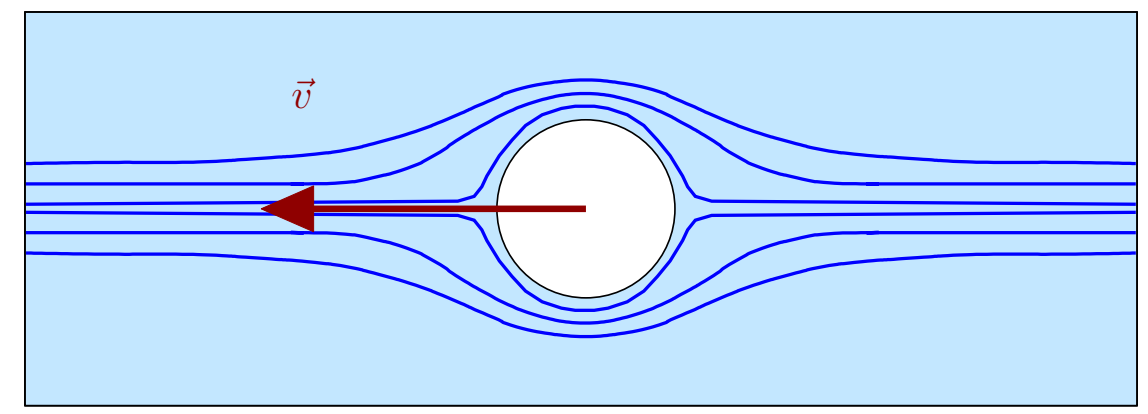

Abbildung 4.1: Darstellung der symmetrischen Potentialströmung um eine durch die Flüssigkeit bewegte Blase. Die durch diese Umströmung auftretende scheinbare Masse wird als „virtuelle“ Masse der Blase bezeichnet und verursacht die „virtuelle“ Trägheit.

Im Spezialfall einer sphärischen Blase entspricht die virtuelle Masse gerade der Hälfte der verdrängten Flüssigkeitsmasse wie zum Beispiel von LANDAU und Lifschitz [69] oder Leighton [7] angegeben:

$$
\vec{F}_{\mathrm{M}}=\frac{\varrho_{1} V}{2} \ddot{\vec{x}}
$$

$\varrho_{1} \quad$ Dichte der Flüssigkeit

$V \quad$ Blasenvolumen

$\ddot{\vec{x}} \quad$ Beschleunigung der Blase

${ }^{1}$ Eine symmetrische, Potential- oder auch Laplace-Strömung ergibt sich bei hoher ReynoldsZahl und wirbelfreier Strömung als Lösung der viskositätsfreien Navier-Stokes-Gleichung. Bei langsam bewegten Objekten mit niedriger Reynolds-Zahl stellt sich eine ebenfalls symmetrische schleichende Strömung ein, wie zum Beispiel von BATChELOR [67] oder TRITTON [68] angegeben.

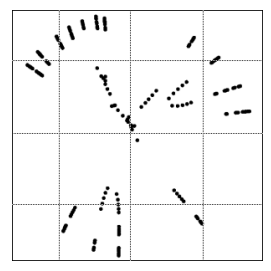




\subsection{Primäre Bjerkneskraft}

Die primäre Bjerkneskraft beschreibt die Kraftwirkung auf eine Blase durch das am Ort der Blase vorherrschende äußere Schallfeld:

$$
\vec{F}_{\mathrm{Bp}}=\vec{F}_{\mathrm{Bp}}\left(t, \tilde{p}, R_{\mathrm{n}}\right)
$$

$$
\begin{aligned}
t & \text { Zeit } \\
\tilde{p}=\tilde{p}(\vec{x}, t) & \text { Schallwechseldruck } \\
R_{\mathrm{n}} & \text { Ruheradius der Blase }
\end{aligned}
$$

Von BJERKNes [70] wird die Kraftwirkung als Resultat des Schallfeldgradienten erklärt. Mit der in Abbildung 4.2 gezeigten Variation des Druckes über die Ausdehnung der Blase wirken unterschiedliche Drücke auf die einzelnen Punkte der Blasenoberfläche.

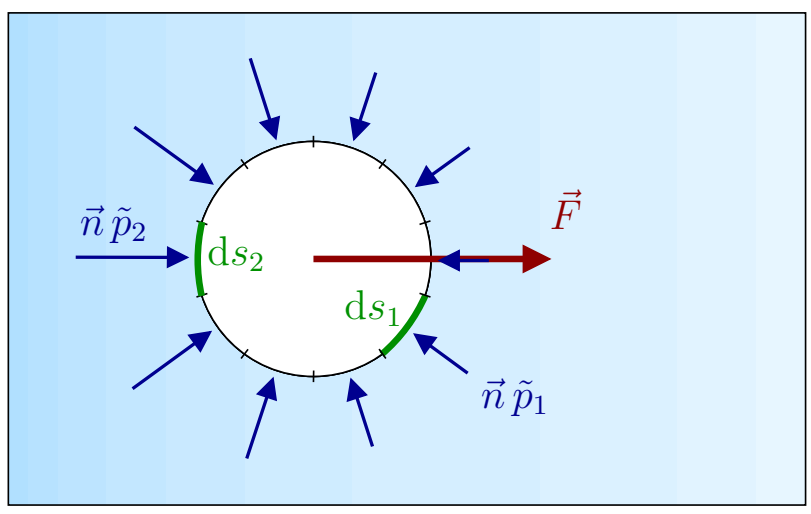

Abbildung 4.2: Im Gradientenfeld des Schalls (Farbverlauf) erfahren die Oberflächenelemente der Blase unterschiedlich starke Kräfte, die im Resultat eine Beschleunigung in Richtung des (negativen) Druckgradienten bewirken.

Integriert über die Oberfläche ergibt sich die wirkende Kraft mit Ausnutzung des Gauß'schen Satzes. Das negative Vorzeichen ergibt sich aus der Orientierung der Normalenvektoren:

$$
\vec{F}=\int_{\partial V} \vec{n} \tilde{p} \mathrm{~d} s=-\int_{V} \nabla \tilde{p} \mathrm{~d} V
$$

$\mathrm{d} s$ Oberflächenelement

$\partial V$ Blasenoberfläche

$\vec{n} \quad$ Flächennormalenvektor in die Blase gerichtet

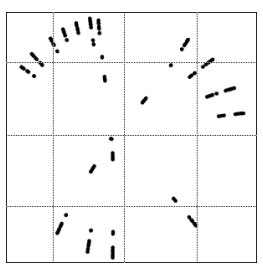


Für Schallfelder im Kilohertzbereich ist die Wellenlänge $(\lambda \approx 10 \mathrm{~cm})$ deutlich größer als der Durchmesser einer typischen Kavitationsblase $\left(R_{\max } \approx 0.1 \mathrm{~cm}\right)$, in diesem Fall kann der Druckgradient als konstant über das Integrationsgebiet angenommen werden:

$$
\vec{F} \approx-\nabla \tilde{p} \int_{V} \mathrm{~d} V=-V \nabla \tilde{p}
$$

Von einem harmonisch oszillierenden Schallfeld ausgehend wird vereinfachend eine periodische Volumenoszillation angenommen, mit einer Periodizität ${ }^{2}$ von $n_{\text {per }}$. Die gemittelte Kraft über einen Pulsationszyklus der Blasen $\tau_{\text {zyk }}=n_{\text {per }} / \nu$ wird als primäre Bjerkneskraft bezeichnet:

$$
\begin{aligned}
& \qquad \vec{F}_{\mathrm{Bp}}=-\langle V \nabla \tilde{p}\rangle_{\tau_{\text {zyk }}} \\
& n_{\text {per }} \begin{array}{l}
\text { Periodizität der Blasenpulsation } \\
\tau_{\text {osz }}=1 / \nu
\end{array} \\
& \tau_{\text {zyk }}=\tau_{\text {osz }} n_{\text {per }} \text { Periodendauer der Schallfeldoszillation } \\
& \text { Periodendauer eines Blasenpulsationszyklus }
\end{aligned}
$$

Die Annahme eines harmonisch treibenden Schallfeldes mit ortsabhängiger Amplitude und Phase führt auf die Schreibweise (3.2):

$$
\tilde{p}=p_{\mathrm{A}}(\vec{x}) \sin (\omega t-\varphi(\vec{x}))
$$

Durch die phasenstarre Kopplung des die radiale Pulsation antreibenden akustischen Wechseldruckes mit gerade dieser Pulsation entfällt in der periodenweisen Mittelung die Phasenlage:

$$
\vec{F}_{\mathrm{Bp}}=-\left\langle\nabla p_{\mathrm{A}}(\vec{x}) \sin (\omega t) V(t)\right\rangle_{\tau_{\mathrm{zyk}}}+\left\langle p_{\mathrm{A}}(\vec{x}) \nabla \varphi(\vec{x}) \cos (\omega t) V(t)\right\rangle_{\tau_{\mathrm{zyk}}}
$$

Die rein ortsabhängigen Terme lassen sich aus der zeitlichen Mittelung herausziehen:

$$
\vec{F}_{\mathrm{Bp}}=-\nabla p_{\mathrm{A}}(\vec{x})\langle\sin (\omega t) V(t)\rangle_{\tau_{\mathrm{zyk}}}+p_{\mathrm{A}}(\vec{x}) \nabla \varphi(\vec{x})\langle\cos (\omega t) V(t)\rangle_{\tau_{\mathrm{zyk}}}
$$

Die beiden verbleibenden Mittelungen bilden Koeffizienten der primären Bjerkneskraft. Damit ist die Zeitabhängigkeit separiert und die primäre Bjerkneskraft kann ortsabhängig angegeben werden:

$$
\begin{aligned}
f_{\mathrm{Bp}}^{\nabla p_{\mathrm{A}}} & \equiv\langle\sin (\omega t) V\rangle_{\tau_{\mathrm{zyk}}} \\
f_{\mathrm{Bp}}^{\nabla \varphi} & \equiv\langle\cos (\omega t) V\rangle_{\tau_{\mathrm{zyk}}}
\end{aligned}
$$

2 Die Periodizität wird auf 16 begrenzt, das heißt $n_{\text {per }}>16$ steht in dieser Arbeit für höher periodische Blasenpulsationen sowie für mögliche chaotische Schwingungsformen; Abbildung 2.4 zeigt die Periodizitätenverteilung in der $R_{\mathrm{n}}-p_{\mathrm{A}}$-Parameterebene. 


$$
\Rightarrow \quad \vec{F}_{\mathrm{Bp}}=-\nabla p_{\mathrm{A}}(\vec{x}) f_{\mathrm{Bp}}^{\nabla p_{\mathrm{A}}}+p_{\mathrm{A}}(\vec{x}) \nabla \varphi(\vec{x}) f_{\mathrm{Bp}}^{\nabla \varphi}
$$

Die Betrachtung des harmonischen Schallwechseldruckes lässt sich in die beiden idealisierten Grenzfälle von stehender und laufender ${ }^{3}$ ebener Welle aufteilen:

$$
\begin{aligned}
\text { stehend: } \quad p_{\mathrm{A}}=\sin (\vec{k} \vec{x}) & \Rightarrow \nabla p_{\mathrm{A}}=\vec{k} \cos (\vec{k} \vec{x}) \\
\varphi=\mathrm{const} . & \Rightarrow \nabla \varphi=0 \\
\text { laufend: } \quad p_{\mathrm{A}}=\mathrm{const.} & \Rightarrow \nabla p_{\mathrm{A}}=0 \\
\varphi=\vec{k} \vec{x} & \Rightarrow \nabla \varphi=\vec{k}
\end{aligned}
$$

$\lambda$ Wellenlänge

$k=2 \pi / \lambda \quad$ Wellenzahl

Abbildung 4.3 zeigt exemplarisch die beiden idealen Fälle einer stehenden Welle $\left(\hat{p}_{\mathrm{A}}^{\nabla p_{\mathrm{A}}}=120 \mathrm{kPa}\right)$ und einer von links einlaufenden Welle $\left(\hat{p}_{\mathrm{A}}^{\nabla \varphi}=110 \mathrm{kPa}\right)$ sowie ihre Überlagerung. Im reinen stehenden Wellenfeld zeigt sich dabei die bekannte zum Druckbauch gerichtete Kraftwirkung. Mit dem laufenden Anteil wird die Blase von der Welle mitgenommen, das heißt entlang des Phasengradienten beschleunigt. Durch die Superposition mit dem konstanten laufenden Anteil kommt es im Bereich $x<0$ zu einer Verstärkung der primären Bjerkneskraft gegenüber dem reinen stehenden Anteil. Mit dem gleichen Versatz der Kraft wird für $x>0$ die anziehende Kraft in ihrem Maximalwert geschwächt. Bei den gewählten Parametern resultiert eine Verschiebung der Gleichgewichtslage um etwa $0.24 \mathrm{~mm}$. Außerdem tritt neben der ursprünglichen stabilen Gleichgewichtslage der stehenden Komponente bei $x=0$ eine instabile Gleichgewichtslage bei $x \approx 7 \mathrm{~mm}$ auf. Durch dieses zweite Gleichgewicht wird der anziehende Bereich des Druckbauches zu großen $x$ hin begrenzt.

Die Wirkung der primären Bjerkneskraft ist grundlegend für die Ausbildung vieler Blasenstrukturen. Im Stehwellenfeld ergeben sich je nach Vorzeichen anziehende und abstoßende Bereiche.

Bereits in Abbildung 2.2 wurde das Schwingungsverhalten von Blasen in einem Stehwellenfeld verschiedener Schalldruckamplituden untersucht. So folgt eine schwach getriebene Blase im Wesentlichen dem treibenden Schalldruck mit einer Phasenverschiebung um $180^{\circ}$. Das heißt, die Blase wird in der Unterdruckphase expandiert und in der Überdruckphase komprimiert. Mit steigender Schalldruckamplitude kommt es neben einem Zunehmen des erreichten maximalen Blasenradius gleichzeitig zu einer Verschiebung der Phasenlage der größten erreichten

${ }^{3}$ Die Laufrichtung ergibt sich aus der Richtung des Wellenzahlvektors. $k_{\mathrm{x}, \mathrm{y}, \mathrm{z}}>0$ beschreibt eine in positiver Richtung laufende Welle.

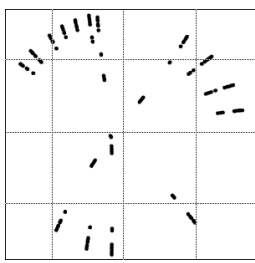




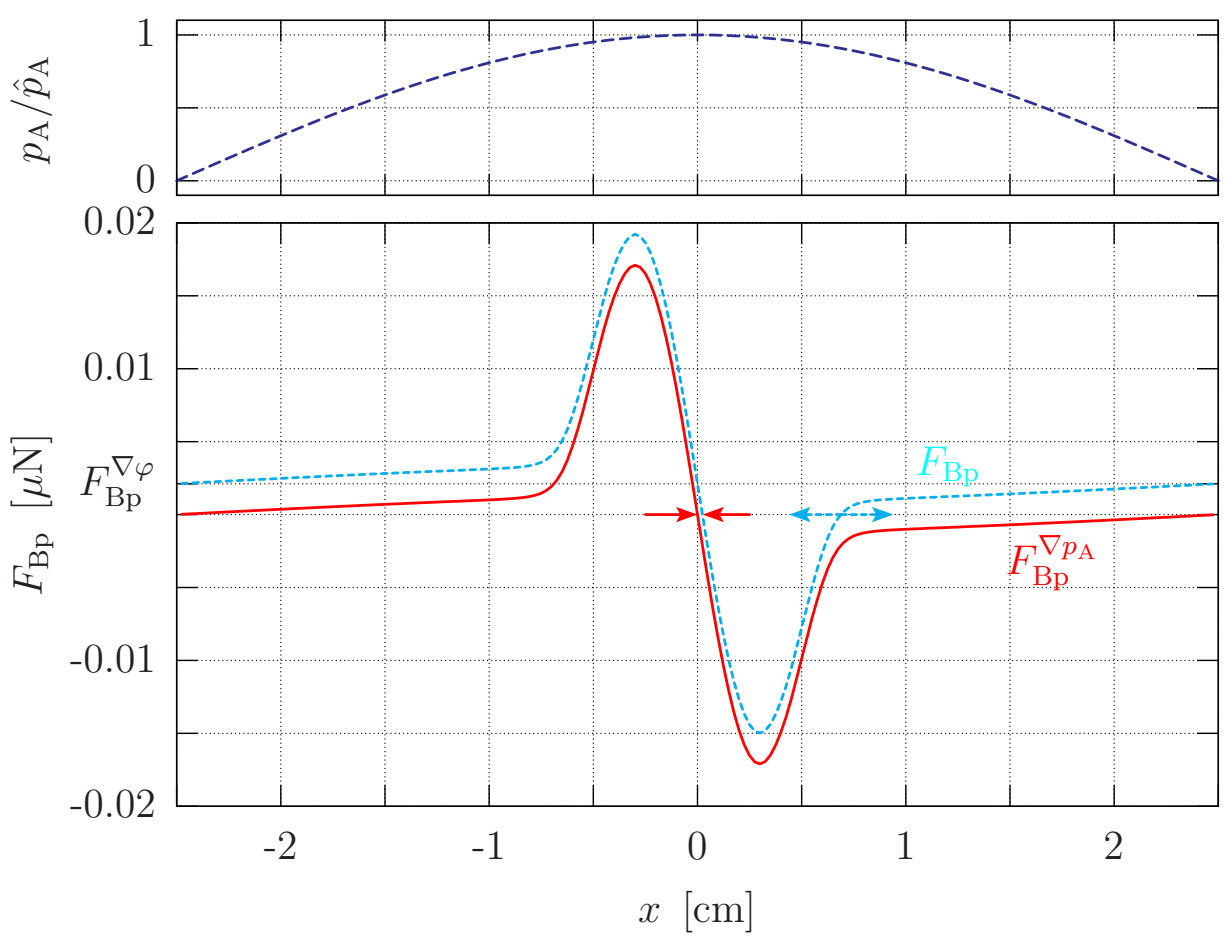

Abbildung 4.3: Komponentenweise Betrachtung der primären Bjerkneskraft (4.4). Durch die Überlagerung des stehenden Anteils (entsprechend der oben gezeigten Schalldruckamplitude) mit dem laufenden Anteil (konstante Schalldruckamplitude) bildet sich die durch die Pfeile gekennzeichnete instabile Gleichgewichtslage der Gesamtkraft aus $F_{\mathrm{Bp}}=F_{\mathrm{Bp}}^{\nabla p_{\mathrm{A}}}+F_{\mathrm{Bp}}^{\nabla \varphi}$; $\lambda=10 \mathrm{~cm}, C_{\mathrm{l}}=1482 \mathrm{~m} / \mathrm{s}, \nu=C_{\mathrm{l}} / \lambda, R_{\mathrm{n}}=5 \mu \mathrm{m}, \hat{p}_{\mathrm{A}}^{\nabla p_{\mathrm{A}}}=120 \mathrm{kPa}$, $\hat{p}_{\mathrm{A}}^{\nabla \varphi}=110 \mathrm{kPa}$.

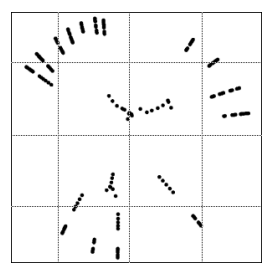


Blasenexpansion. Abhängig von dieser Phasenbeziehung zwischen der Blasenpulsation $V(t)$ und dem Schalldruckverlauf $\nabla \tilde{p}(t)$ kann es zu einer Umkehr des Vorzeichens der primären Bjerkneskraft kommen (AкHATOV et al. [71]), wie es Abbildung 4.4 zeigt.

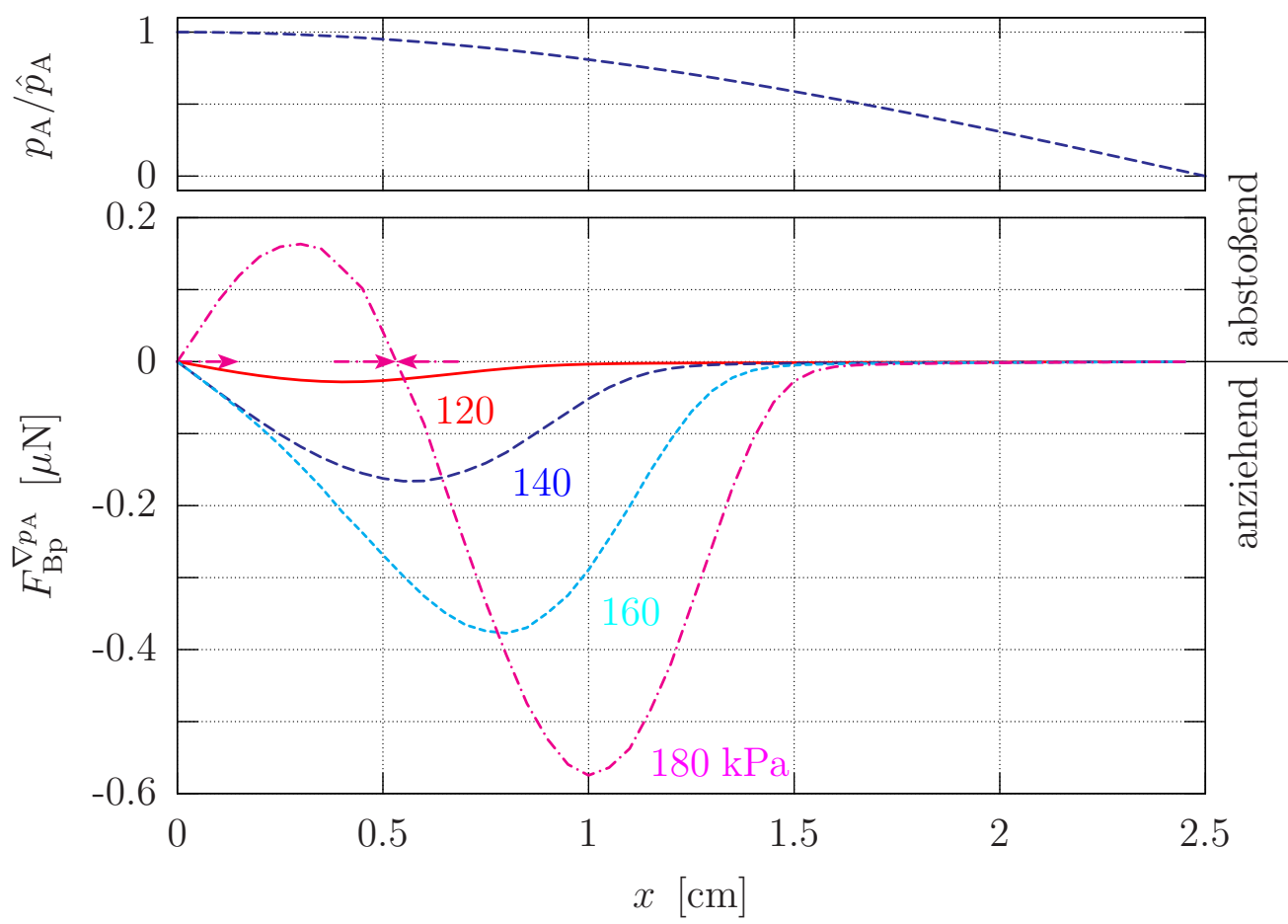

Abbildung 4.4: Wirkung des Stehwellenanteils der primären Bjerkneskraft in einem kosinusförmigen Stehwellenfeld auf eine Blase bei verschiedenen maximalen Schalldruckamplituden; der Druckbauch liegt bei $x=0$ $\hat{p}_{\mathrm{A}}=120,140,160,180 \mathrm{kPa}, \lambda=10 \mathrm{~cm}, \nu=20 \mathrm{kHz}, \eta=1, R_{\mathrm{n}}=5 \mu \mathrm{m}$.

Für typische Werte von Frequenz, Druckamplitude und Blasenradius ist der Verlauf der primären Bjerkneskraft abhängig vom Abstand zum Druckbauch und dem maximalen Schalldruck in Abbildung 4.4 gezeigt. Mit anwachsender Schalldruckamplitude verstärkt sich die radiale Blasenpulsation und damit die anziehende Wirkung des Druckbauchs, bis die nichtlineare Dynamik der Blase zu dem Vorzeichenwechsel in der Kraft führt. Mit diesem Vorzeichenwechsel bildet sich die in Abbildung 4.4 für $\hat{p}_{\mathrm{A}}=180 \mathrm{kPa}$ eingezeichnete stabile Gleichgewichtslage aus (hier nahe $x=0.5 \mathrm{~cm}$ ). Für diesen Schalldruck stellt der Druckbauch $(x=0 \mathrm{~cm})$ eine instabile Gleichgewichtslage dar. Er wirkt daher abstoßend. ${ }^{4}$

4 Die stabile Gleichgewichtslage durch die anziehende Wirkung des Druckbauchs bei den gezeigten niedrigeren Schalldruckamplituden wird gerade ausgenutzt um etwa „Blasenfallen“ zu bauen.

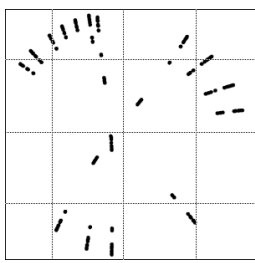


Abbildung 4.5 zeigt das Vorzeichen des primären Bjerkneskraftkoeffizienten $f_{\mathrm{Bp}}^{\nabla p_{\mathrm{A}}}$ bei einer Frequenz von $\nu=20 \mathrm{kHz}$ in der Parameterebene von Schalldruckamplitude und Ruheradius. In dem „abstoßenden“ Bereich werden die Blasen gemäß der primären Bjerkneskraft (4.4) gegen den Druckamplitudengradienten zu niedrigen Schalldrücken hingetrieben. Dagegen werden sie in dem „anziehenden“ Bereich zu höheren Drücken gezogen.

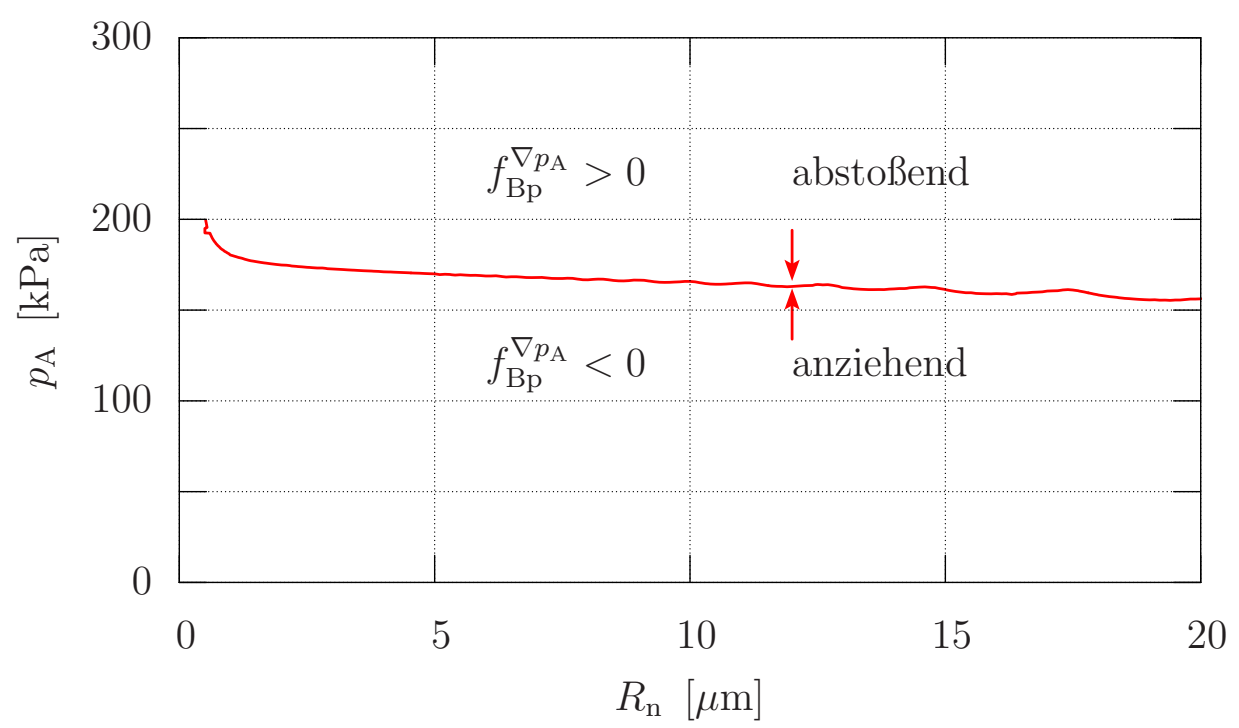

Abbildung 4.5: Vorzeichenwechsel des Stehwellenanteils des primären Bjerkneskraftkoeffizienten $f_{\mathrm{Bp}}^{\nabla p_{\mathrm{A}}}$. Unterhalb der eingezeichneten Linie werden die jeweiligen Blasen zum Druckbauch hin gezogen, für Druckamplituden oberhalb wirkt der Druckbauch abstoßend; $\nu=20 \mathrm{kHz}$.

Die Betrachtung des durch die Schalldruckamplitude und den Ruheradius aufgespannten Parameterraums lässt unter Einbeziehung der in Kapitel 2 eingeführten Abgrenzungen einen Stabilitätsbereich erkennen, in dem Kavitationsblasen längere Zeit existieren können. In Abbildung 4.6 sind dazu neben dem Vorzeichenwechsel des primären Bjerkneskraftkoeffizienten aus Abbildung 4.5 auch die Blake-Schwelle für $\Omega=2$ entsprechend Abbildung 2.8 eingezeichnet. Ebenfalls gezeigt ist die Grenze parametrischer Oberflächenstabilität für $\max (|\lambda|)=1$ der $l=2$ Mode nach Abbildung 2.13 sowie das Gleichgewicht der gerichteten Diffusion von Stickstoffmolekülen bei vollständiger Sättigung $c_{\infty} / c_{\text {sat }}=100 \%$ aus Abbildung 2.20.

Zu kleinen Ruheradien hin laufen die Diffusionsgrenze und die Blake-Schwelle zusammen, wie es auch Rechnungen von Louisnard und Gomez [72] gezeigt haben. Für den Diffusionsstrom ist die Größe der Oberfläche entscheidend, so dass

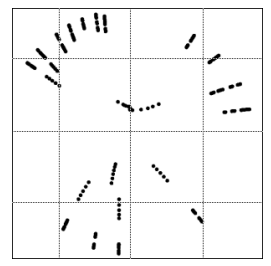




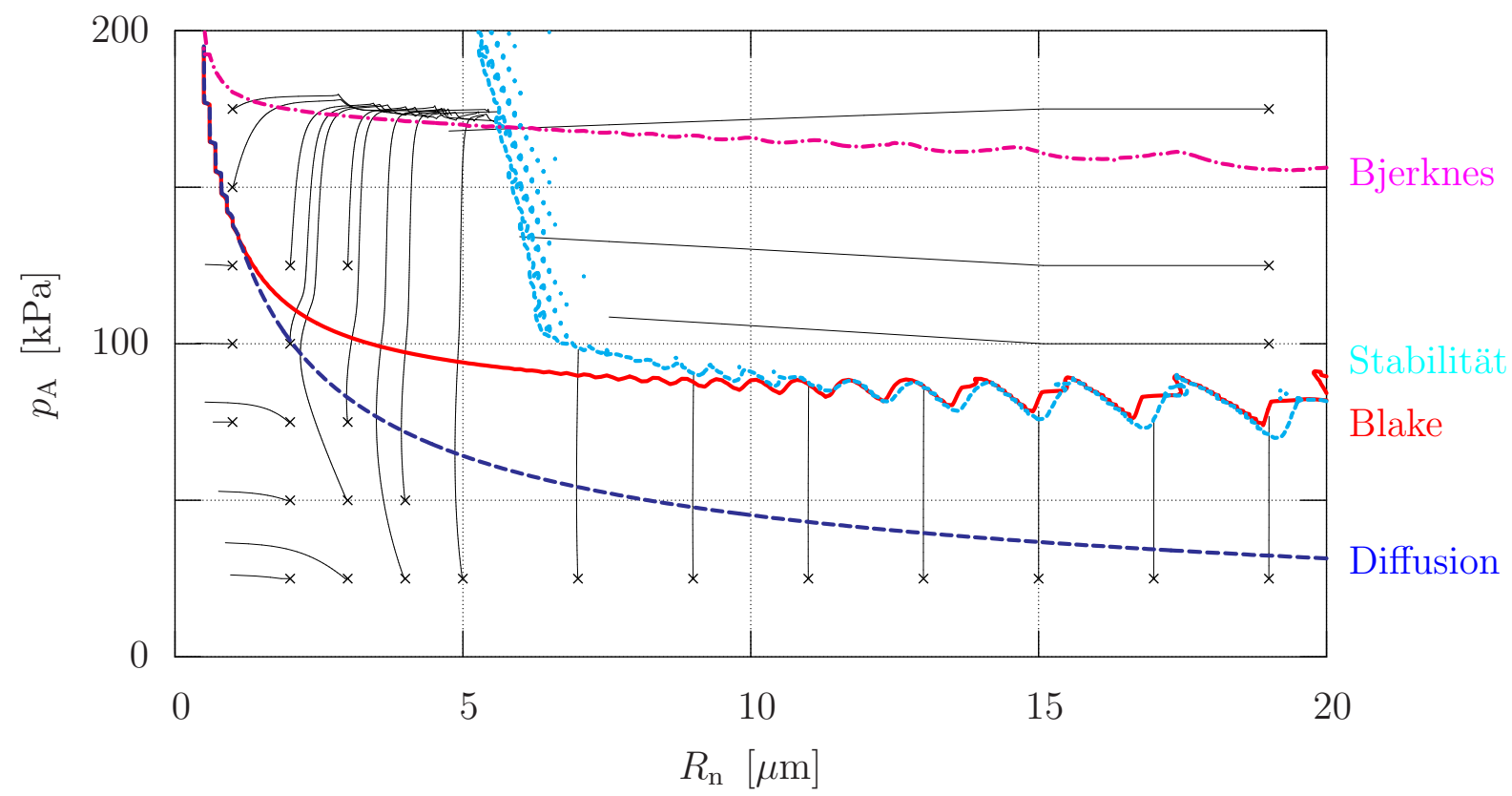

Abbildung 4.6: Darstellung des „Lebensraums“ von Kavitationsblasen in der Parameterebene der Schalldruckamplitude und des Ruheradius; Aufgetragen ist die dynamische Blake-Schwelle $R_{\max } \geq \Omega \cdot R_{\mathrm{n}}$ die sich aus Betrachtung der Lösung der Keller-Miksis-Gleichung (2.10) ergibt, das Diffusionsgleichgewicht (2.36) von Stickstoffmolekülen, die parametrische Stabilitätsgrenze der $l=2$ Mode für deren Lösung (2.22) an das KellerMiksis-Modell gekoppelt wird sowie der Vorzeichenwechsel des primären Bjerkneskraftkoeffizienten (4.3a); $\nu=20 \mathrm{kHz}, c_{\infty} / c_{\mathrm{sat}}=100 \%, \Omega=2$.

Die von den Kreuzen ausgehenden Bahnen zeigen die Bewegung von Blasen im entsprechenden Parameterbereich, die sich in einem analytisch definierten Stehwellenfeld (3.6) bewegen; $\hat{p}_{\mathrm{A}}=200 \mathrm{kPa}, \lambda=10 \mathrm{~cm}$.

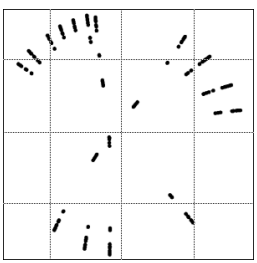


es für eine kleine Kavitationsblase einer starken radialen Dynamik bedarf, um die Grenze diffusionsbedingten Anwachsens zu erreichen. Zu großen Ruheradien hin bildet die Blake-Schwelle eine Abschätzung der parametrischen Stabilitätsgrenze, da große Blasen schon bei relativ schwacher Expansion oberflächeninstabil werden.

Mit dem Überschreiten der Blakeschwelle hin zu stärkeren Schalldruckamplituden setzt die typische nichtlineare Pulsationsdynamik der Kavitationsblasen ein. Damit erreichen sie deutlich größere Maximalradien und erfahren eine stärkere Anziehung der primären Bjerkneskraft, bis sie schließlich die Grenze des Vorzeichenwechsels in dem primären Bjerkneskraftkoeffizienten erreichen.

Die Bewegung entlang der $p_{\mathrm{A}}$-Richtung in der Parameterebene ist dominierend. Sie entspricht einer tatsächlichen Translation einer Blase im Stehwellenfeld. Diffusionsbedingte Verschiebungen gemäß der Wachstumsrate (2.35) entlang der $R_{\mathrm{n}^{-}}$ Achse laufen auf deutlich langsameren Zeitskalen gegenüber der Translation ab. Oberhalb der Diffusionsschwelle „verschieben“ sich die Blasen dabei zu größeren Ruheradien, unterhalb tendieren sie zum Aufösen. Sie können jedoch durch die primäre Bjerkneskraft auch im Fall kleiner Ruheradien noch über die Diffusionsschwelle gezogen werden (im Diagramm vertikal nach oben). Ebenso werden Blasen, die oberhalb der Diffusionsgrenze zu großen Ruheradien hin anwachsen, durch die primäre Bjerkneskraft in den parametrisch instabilen Bereich gezogen. Kavitationsblasen, die die Grenze der parametrischen Oberflächenstabilität überschreiten, fragmentieren, so dass es zu einem „Springen“ entlang der $R_{\mathrm{n}}$-Achse zu niedrigeren Ruheradien kommt.

Im Ganzen kann durch die zusammengeführten Betrachtungen in Auftragung 4.6 für $\nu=20 \mathrm{kHz}$ ein Bereich zwischen $R_{\mathrm{n}} \approx 1.5 \ldots 6 \mu \mathrm{m}$ und $p_{\mathrm{A}} \approx 100 \ldots 175 \mathrm{kPa}$ ausgemacht werden, in dem der Aufenthalt von Kavitationsblasen durch alle Effekte begünstigt ist bzw. längerfristig möglich sein sollte. Falls der Anregungsdruck im Druckbauch höher als ca. $175 \mathrm{kPa}$ ist, bleiben die Blasen außerhalb des Bauches bei diesem Wert „hängen“, so dass dann gewissermaßen ein Akkumulationspunkt in der Parameterebene bei etwa $R_{\mathrm{n}} \approx 6 \mu \mathrm{m}$ und $p_{\mathrm{A}} \approx 175 \mathrm{kPa}$ existieren sollte.

Mit dieser Analyse ist eine mögliche Erklärung für das vorrangig beobachtete Auftreten von Blasen im Bereich von $R_{\mathrm{n}} \approx 5 \mu \mathrm{m}$ gegeben. Außerdem zeigt sich, das Blasen unterhalb der Diffusionsschwelle, sich nicht zwangsweise aufösen müssen. Bei hinreichendem Einfluss der primären Bjerkneskraft ist es möglich dass sie die Schwelle überschreiten bevor sie vollständig in Lösung gegangen sind. Somit existiert neben der Vereinigung von Mikroblasen ein weiterer Mechanismus, der

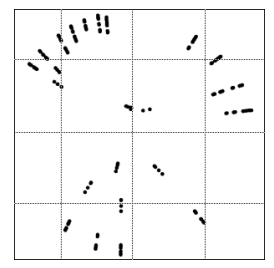


ihr Anwachsen bewirkt und damit von Bedeutung für die Strukturbildung ist.

Die Abbildungen 4.7 und 4.8 zeigen die möglichen stabilen Gleichgewichtslagen im Stehwellenfeld einer Blase mit $R_{\mathrm{n}}=5 \mu \mathrm{m}$. Betrachtet sind dabei die Schalldruckverteilungen der $(1,1,1)$-Mode bei $\nu=23.64 \mathrm{kHz}$ sowie die $(1,1,2)$-Mode bei $\nu=33.86 \mathrm{kHz}$, die sich gemäß Abschnitt 3.2 als Lösungen der gedämpften Helmholtz-Gleichung (3.4) bestimmen. Dieser Betrachtung liegt die Geometrie der in Abschnitt 3.2 vorgestellten quaderförmigen Hellma-Küvette $\left(5 \times 5 \times 5 \mathrm{~cm}^{3}\right)$ zugrunde.
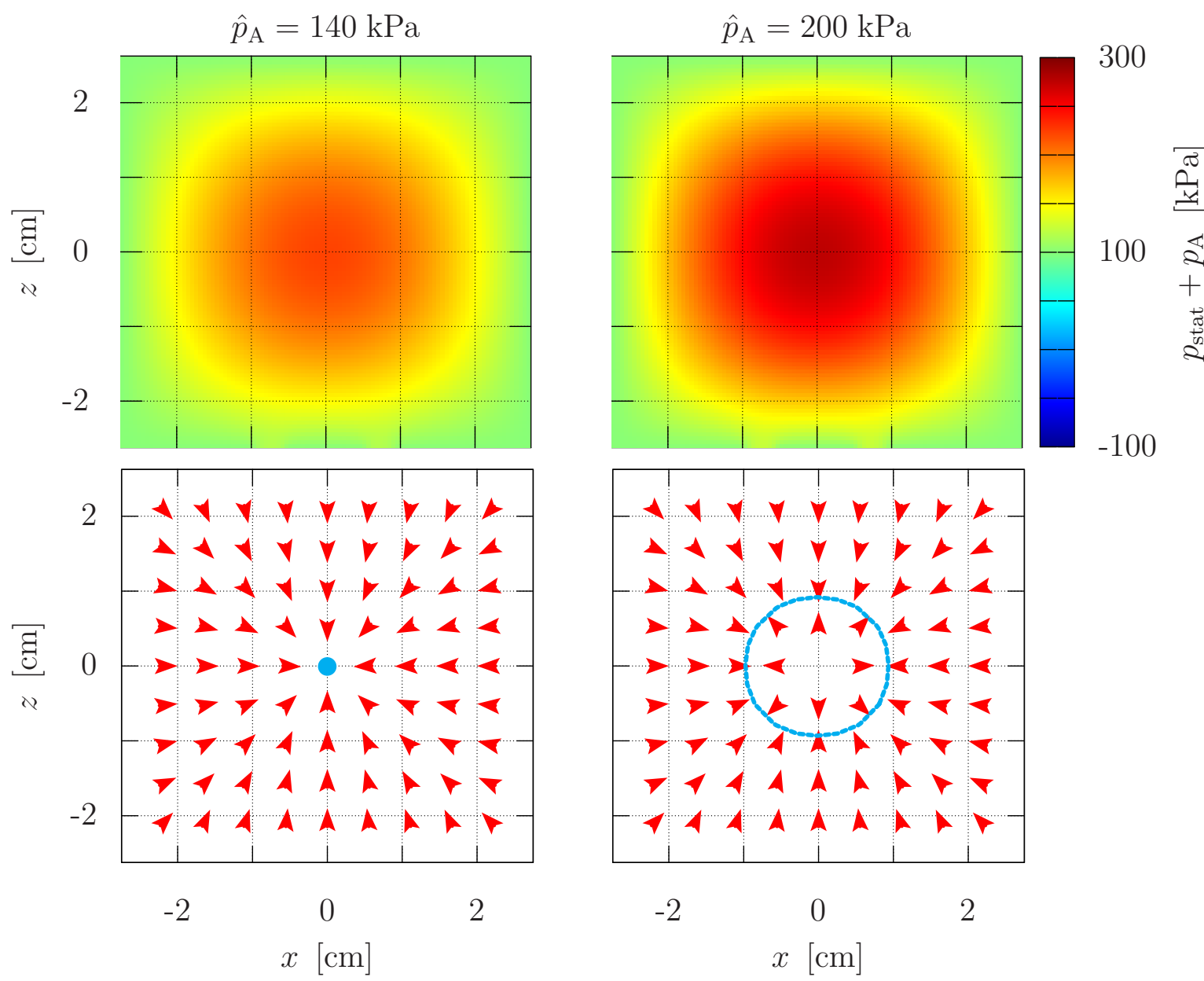

Abbildung 4.7: Oben ist die aus Abbildung 3.4 bekannte (1, 1, 1)-Mode der Hellma-Küvette zu zwei verschiedenen maximalen Schalldruckamplituden gezeigt. Die unteren Auftragungen zeigen die zugehörige Wirkungsrichtung der primären Bjerkneskraft auf eine $5 \mu \mathrm{m}$-Blase. Hervorgehoben sind die stabilen Gleichgewichtslagen der Blase; $\nu=23.647 \mathrm{kHz}$, $\hat{p}_{\mathrm{A}}=140,200 \mathrm{kPa}$.

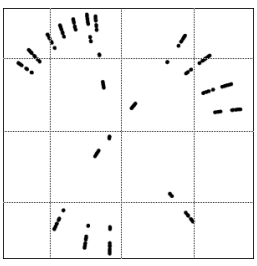



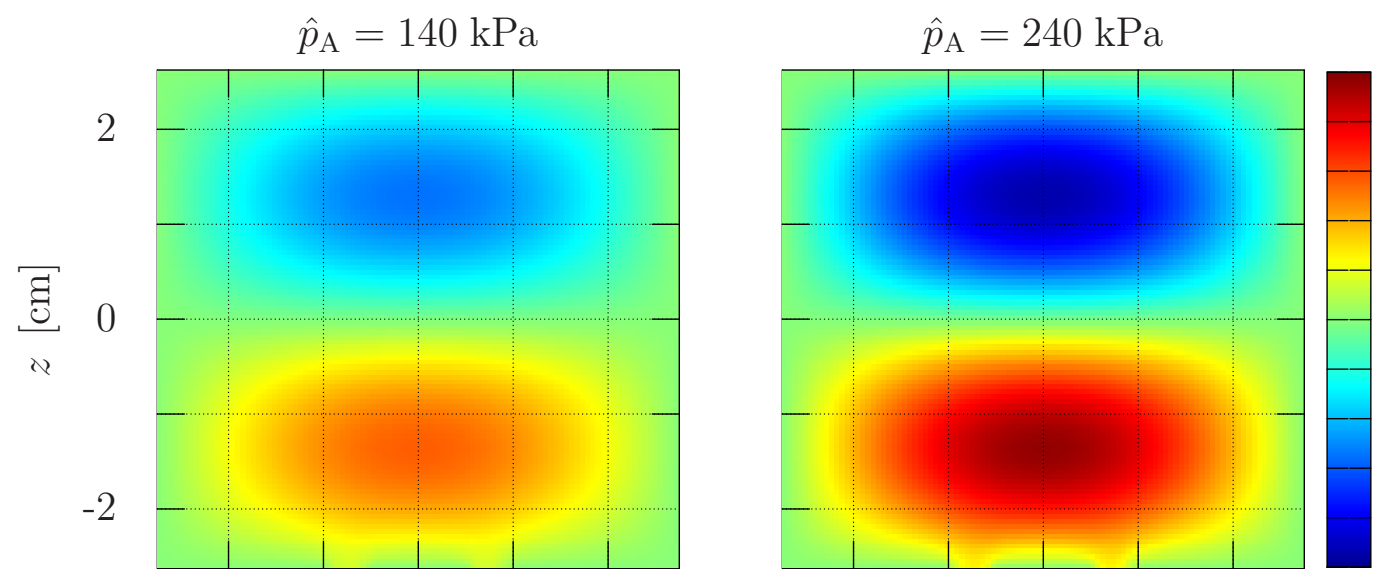

300
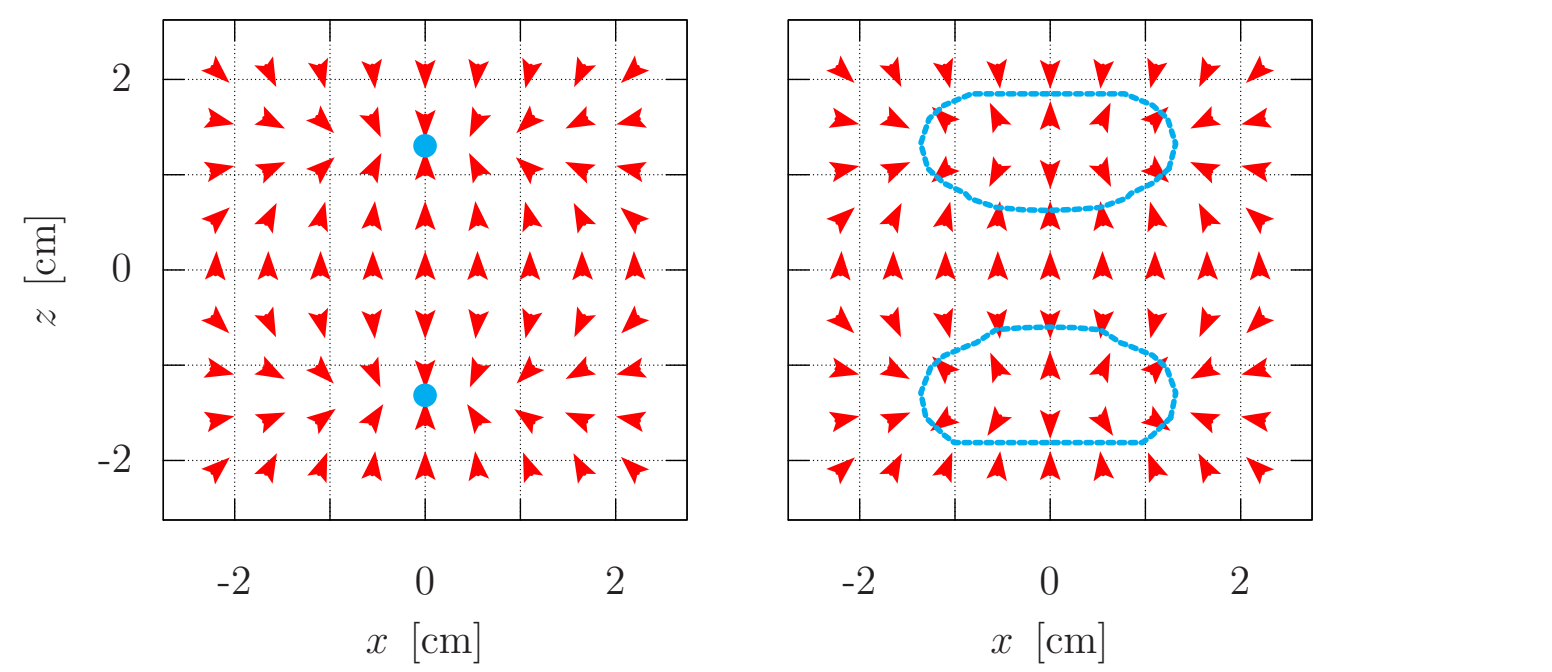

Abbildung 4.8: Oben ist die aus Abbildung 3.4 bekannte (1, 1, 2)-Mode der Hellma-Küvette zu zwei verschiedenen maximalen Schalldruckamplituden gezeigt. Die unteren Auftragungen zeigen die zugehörige Wirkungsrichtung der primären Bjerkneskraft auf eine $5 \mu \mathrm{m}$-Blase. Hervorgehoben sind die stabilen Gleichgewichtslagen der Blase; $\nu=33.86 \mathrm{kHz}$, $\hat{p}_{\mathrm{A}}=140,240 \mathrm{kPa}$.

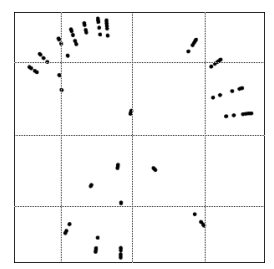


Im laufenden Wellenfeld, etwa unter einer Sonotrode, tritt der Phasengradient auf $(\nabla \varphi \neq 0)$, der mit der laufenden Komponente der primären Bjerkneskraft eine den Blasenstrom fokussierende Wirkung haben kann.

Die Amplitudenverteilung des aus Abbildung 3.18 bekannten laufenden Wellenfeldes unter der Sonotrode wird in den oberen Abbildungen 4.9 im Schnitt entlang der $z$-Achse für Amplitude und Phase dargestellt, in den oberen Abbildungen 4.10 entlang der $x$-Achse.

Wie in Abbildung $4.9 \mathrm{zu}$ sehen, bildet sich entlang der Symmetrieachse $(x=0)$ die höchste Druckamplitude aus und der stärkste Gradientenabfall. Bei typischen Schalldrücken einer Sonotrode $\left(\hat{p}_{\mathrm{A}} \gtrsim 200 \mathrm{kPa}\right)$ wird eine $5 \mu \mathrm{m}$-Blase zunächst entlang des Gradienten von der Sonotrodenfläche weg beschleunigt. Gelangt sie in einen Druckbereich, in dem sich analog zu der Betrachtung in Abbildung 4.4 das Vorzeichen des stehenden Anteils der primären Bjerkneskraft umdreht, so befindet sich die Blase in einer stabilen Gleichgewichtslage. Die dritte Auftragung in Abbildung 4.9 zeigt dies Verhalten, außerdem ist zu erkennen, dass an diesem "Gleichgewichtspunkt" die Wirkung des laufenden Anteils der primären Bjerkneskraft überwiegt, und die Blase weiterhin entlang der z-Achse von der Sonotrode weg beschleunigt wird.

Ein Vorzeichenwechsel hin zu einer schwachen Anziehung ist in der untersten Auftragung von Abbildung $4.9 \mathrm{zu}$ sehen. Diese Umkehr verschiebt sich mit zunehmenden Abstand von der Symmetrieachse bei $x=0 \mathrm{~cm}$ in Richtung der Sonotrodenfläche.

Der zweite Effekt im betrachteten laufenden Wellenfeld ist in den Abbildungen 4.10 verdeutlicht. Der Schnitt durch die Schalldruckamplitude entlang der $x$-Achse in verschiedenen Abständen von der Sonotrodenspitze zeigt zum Einen das schon in Abbildung 4.9 gesehene schnelle Absinken entlang der z-Achse, in Verbindung mit einem „Zerlaufen“ der Wellenfront. Zum Anderen bildet sich direkt unterhalb der Sonotrodenfläche ein Amplitudengradient aus, der von der Symmetrieachse aus bis zum Rand der Sonotrode auf etwa $30 \%$ abfällt.

In dem hier angenommenen starken Schalldruck $\hat{p}_{\mathrm{A}}=300 \mathrm{kPa}$ werden typische Kavitationsblasen mit $R_{\mathrm{n}}=5 \mu \mathrm{m}$ von der Symmetrieachse weg beschleunigt. In der untersten Auftragung von Abbildung 4.10 ist eine Vorzeichenumkehr der primären Bjerkneskraft am Rand der Sonotrodenfläche zu erkennen. Zudem zieht sich der stark abstoßende Bereich mit zunehmendem Abstand von der Sonotrode um die Symmetrieachse zusammen.

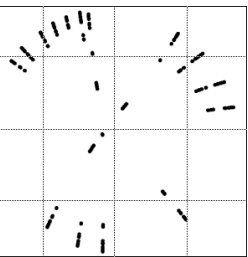




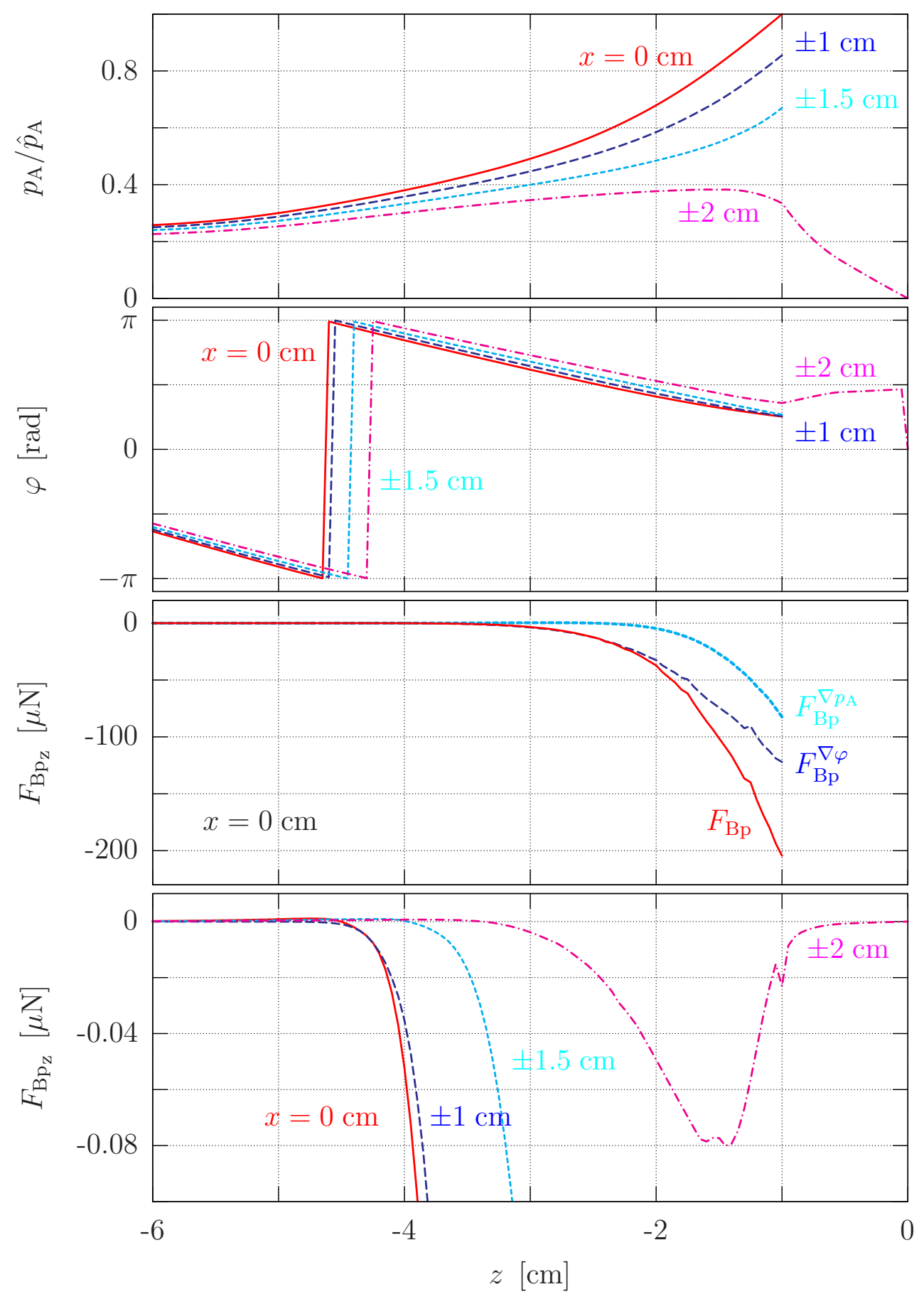

Abbildung 4.9: Schnittdarstellung des Amplituden- und Phasenverlaufs in $z$-Richtung aus Abbildung 3.17 in verschiedenen Abständen zur Symmetrieachse $(x=0 \mathrm{~cm})$. In der dritten Auftragung ist die primäre Bjerkneskraft anteilsweise aufgetragen. Die unterste Abbildung zeigt die primäre Bjerkneskraft in unterschiedlichen Abständen zur Symmetrieachse $x=0,1,1.5,2 \mathrm{~cm}$; Eintauchtiefe $1 \mathrm{~cm}$, Sonotrodendurchmesser $4 \mathrm{~cm}$, $\nu=20 \mathrm{kHz}, D=3.26 \cdot 10^{5} \mathrm{~kg} / \mathrm{m}^{3} / \mathrm{s}, \hat{p}_{\mathrm{A}}=300 \mathrm{kPa}, R_{\mathrm{n}}=5 \mu \mathrm{m}$.

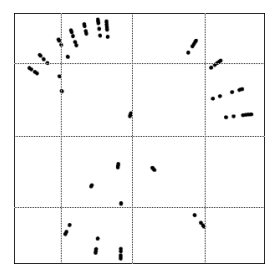




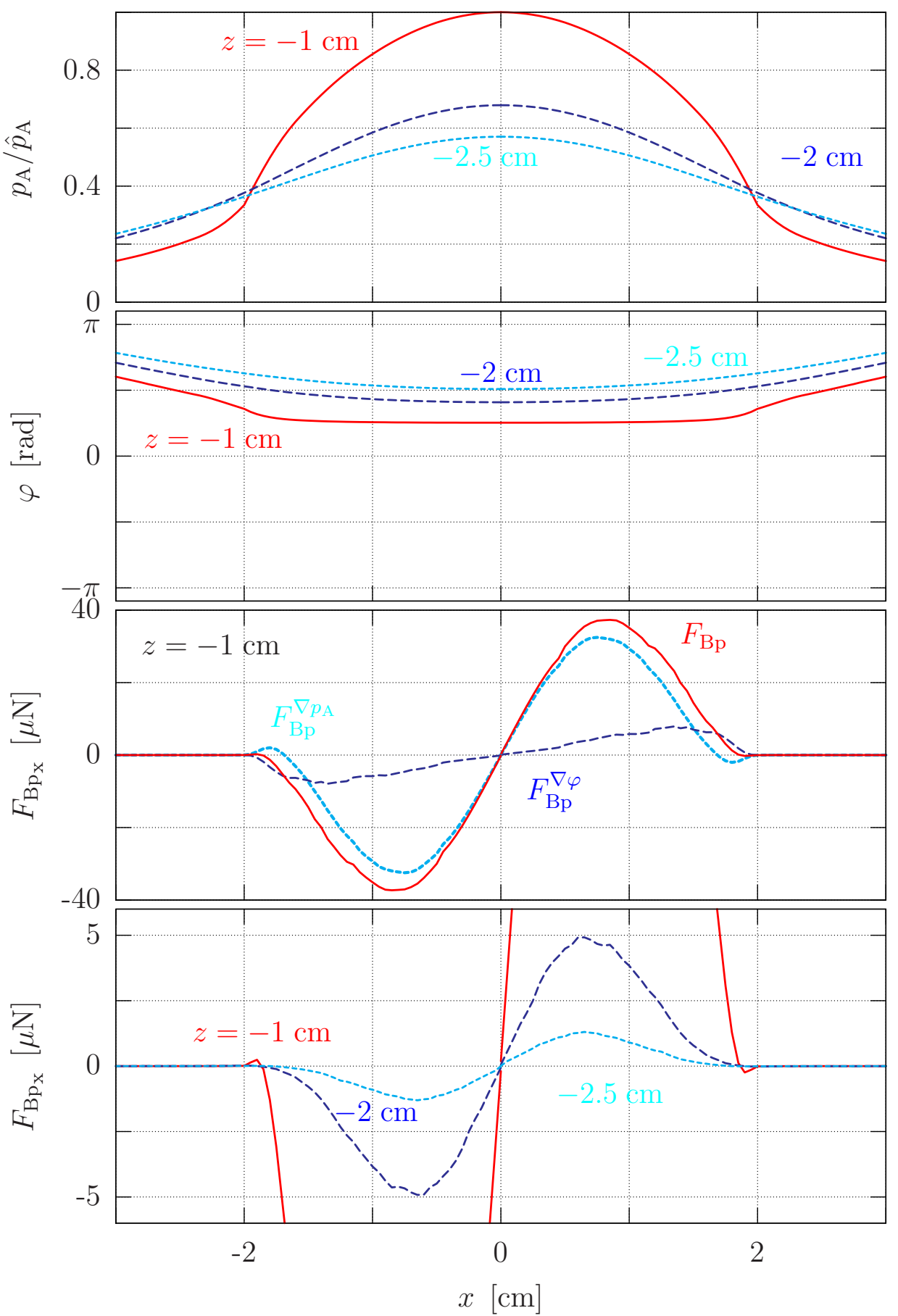

Abbildung 4.10: Schnittdarstellung des Amplituden- und Phasenverlaufs in $x$-Richtung aus Abbildung 3.17 in verschiedenen Abständen zur Stirnfläche der Sonotrode $z=-1,-2,-3 \mathrm{~cm}$. In der dritten Auftragung ist die primäre Bjerkneskraft anteilsweise dargestellt. Die unterste Abbildung zeigt den Verlauf der primären Bjerkneskraft in den verschiedenen $z$-Ebenen; Eintauchtiefe $1 \mathrm{~cm}$, Sonotrodendurchmesser $4 \mathrm{~cm}, \nu=20 \mathrm{kHz}$, $D=3.26 \cdot 10^{5} \mathrm{~kg} / \mathrm{m}^{3} / \mathrm{s}, \hat{p}_{\mathrm{A}}=300 \mathrm{kPa}, R_{\mathrm{n}}=5 \mu \mathrm{m}$.

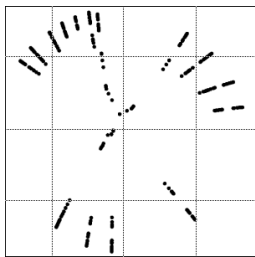




\subsection{Sekundäre Bjerkneskraft}

Eine oszillierende Kavitationsblase induziert eine Druckwelle in die sie umgebende Flüssigkeit. Wie in Abbildung 4.11 skizziert wirkt diese Druckschwankung auf benachbarte Blasen, indem sich der dort herrschende Druckgradient ändert und analog zur primären Bjerkneskraft eine resultierende Kraft auf die Nachbarblase entsteht.

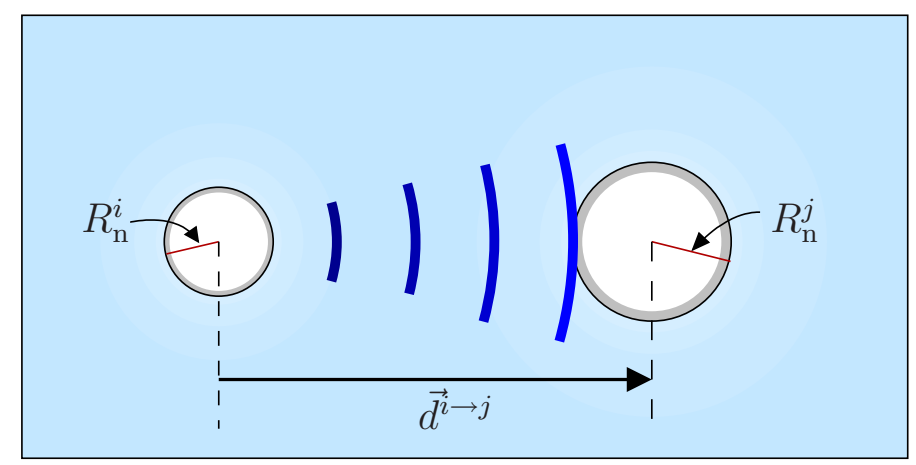

Abbildung 4.11: Schallabstrahlung einer pulsierenden Blase $i$ auf eine benachbarte Blase $j$. Im Allgemeinen haben die Blasen verschiedene Ruheradien und zeigen unterschiedliche Pulsationsdynamiken.

Diese Kraftwirkung durch Blase $i$ auf Blase $j$ wird durch die sekundäre Bjerkneskraft beschrieben:

$$
\vec{F}_{\mathrm{Bs}}^{i \rightarrow j}=\vec{F}_{\mathrm{Bs}}^{i \rightarrow j}\left(p_{\mathrm{A}}^{i}, p_{\mathrm{A}}^{j}, R_{\mathrm{n}}^{i}, R_{\mathrm{n}}^{j}, \vec{d}^{\imath \rightarrow j}\right)
$$

$p_{\mathrm{A}}^{i, j} \quad$ Druck am Ort der Blase $i$ bzw. $j$

$R_{\mathrm{n}}^{i, j} \quad$ Ruheradius der Blase $i$ bzw. $j$

$\overrightarrow{d^{i} \rightarrow j}=\vec{x}^{j}-\vec{x}^{i} \quad$ Abstand der Blasen

Die Laufzeit zwischen den Blasen ist über die Schallgeschwindigkeit mit dem Abstand verknüpft:

$$
\Delta t^{i, j}=\frac{1}{C_{1}}\left|\overrightarrow{d^{\imath} \rightarrow j}\right|
$$

Aus der Kontinuitätsgleichung ergibt sich bei Radialsymmetrie die bereits in Abschnitt 2.1 verwendete Beziehung ${ }^{5}$ für das Geschwindigkeitsfeld um Blase $i$ :

$$
u(t)=\frac{U^{i}\left(t-\Delta t^{i, j}\right) R^{i}\left(t-\Delta t^{i, j}\right)^{2}}{r(t)^{2}}
$$

${ }^{5}$ Hier wird die Schallausbreitung kompressibel gerechnet $\left(C_{1}<\infty\right)$, gleichzeitig aber die Strömung als inkompressibel angenommen ( $\varrho_{1}=$ const.). Diese Näherung ist zulässig für Strömungsgeschwindigkeiten deutlich unterhalb der Schallgeschwindigkeit: $u \ll C_{\mathrm{l}}$.

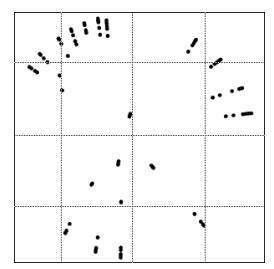


Mit $R^{i}$ und $U^{i}$ sind Radius und Geschwindigkeit an der die Welle abstrahlenden Blasenwand zur Zeit $t-\Delta t^{i, j}$ bezeichnet. Die Größen $r$ und $u$ dagegen beschreiben die durch diese Welle induzierte Bewegung eines Volumenelements zur Zeit $t$ am Ort $r$. Eingesetzt in die Euler-Gleichung ergibt sich:

$$
\begin{aligned}
-\nabla p(t) & =\varrho_{l} \frac{\mathrm{d} \vec{u}}{\mathrm{~d} t} \\
& =\varrho_{1} \frac{1}{r^{2}} \frac{\mathrm{d}}{\mathrm{d} t}\left(\vec{U}\left(t-\Delta t^{i, j}\right) R\left(t-\Delta t^{i, j}\right)^{2}\right)
\end{aligned}
$$

Die Kraftwirkung auf Blase $j$ in diesem durch Blase $i$ induzierten Druckgradienten beträgt ${ }^{6}$ :

$$
\begin{aligned}
\vec{F}^{i \rightarrow j} & =-V^{j}(t) \nabla p(t) \\
& =V^{j}(t) \frac{\varrho_{1}}{4 \pi} \frac{\mathrm{d}^{2}}{\mathrm{~d} t^{2}} V^{i}\left(t-\Delta t^{i, j}\right) \cdot \frac{\vec{d}^{i \rightarrow j}}{\left|\overrightarrow{d^{i \rightarrow j}}\right|^{3}}
\end{aligned}
$$

Nach partieller Integration mit den Anschlussbedingungen $V(0)=V\left(\tau_{\text {zyk }}^{i, j}\right)$ und $\dot{V}(0)=\dot{V}\left(\tau_{\text {zyk }}^{i, j}\right)$ folgt die zeitlich gemittelte sekundäre Bjerkneskraft, die von Blase $i$ auf Blase $j$ wirkt, wie sie etwa auch von METTIN et al. [75] angegeben wird:

$$
\vec{F}_{\mathrm{Bs}}^{i \rightarrow j}=\frac{\varrho_{1}}{4 \pi}\left\langle\dot{V}^{j}(t) \dot{V}^{i}\left(t-\Delta t^{i, j}\right)\right\rangle_{\tau_{\mathrm{zyk}}^{\mathrm{i}, \mathrm{j}}} \frac{\vec{d}^{i \rightarrow j}}{\left|\overrightarrow{d^{i} \rightarrow j}\right|^{3}}
$$

Die Mittelung erfolgt hier über das kleinste gemeinsame Vielfache der Periodizitäten der beiden radial pulsierenden Blasen multipliziert mit der Schallfeldperiodendauer: $\tau_{\mathrm{zyk}}^{i, j}=\operatorname{kgv}\left(n_{\mathrm{per}}^{i}, n_{\mathrm{per}}^{j}\right) \tau_{\mathrm{osz}}$

Die sekundären Bjerkneskräfte mehrerer Blasen überlagern sich linear. Um die gesamte auf Blase $j$ wirkende sekundäre Bjerkneskraft zu erhalten, muss über die ausgeübten Kräfte aller anderen Blasen summiert werden:

$$
\vec{F}_{\mathrm{Bs}}^{j}=\frac{\varrho_{1}}{4 \pi} \sum_{i \neq j}\left\langle\dot{V}^{j}(t) \dot{V}^{i}\left(t-\Delta t^{i, j}\right)\right\rangle_{\tau_{\mathrm{zyk}}^{\mathrm{i}, \mathrm{j}}} \cdot \frac{\overrightarrow{d^{i} \rightarrow j}}{\left|\overrightarrow{d^{i} \rightarrow j}\right|^{3}}
$$

Als sekundärer Bjerkneskoeffizient von Blase $i$ auf Blase $j$ wirkend ist nun

6 Genaugenommen wird durch Blase $i$ auch das Pulsationsverhalten von Blase $j$ beeinflusst, wie zum Beispiel von Kamphausen [73] und Mettin et al. [74] untersucht. Dieser Effekt auf den Radius $R^{j}(t)$ wird aber vernachlässigt.

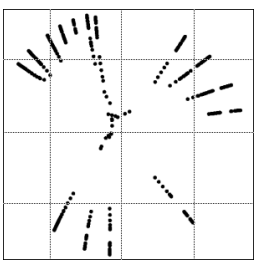


definiert:

$$
\begin{aligned}
f_{\mathrm{Bs}}^{i \rightarrow j} & =\frac{\varrho_{1}}{4 \pi}\left\langle\dot{V}^{j}(t) \dot{V}^{i}\left(t-\Delta t^{i, j}\right)\right\rangle_{\tau_{\mathrm{zyk}}^{\mathrm{i}, \mathrm{j}}} \\
\Rightarrow \quad \vec{F}_{\mathrm{Bs}}^{j} & =\sum_{i \neq j} f_{\mathrm{Bs}}^{i \rightarrow j} \frac{\vec{d}^{i \rightarrow j}}{\left|\overrightarrow{d^{i} \rightarrow j}\right|^{3}}
\end{aligned}
$$

Bei Annahme einer inkompressiblen Flüssigkeit, das heißt unendlicher Schallgeschwindigkeit, besteht keine Laufzeitverzögerung $\left(\Delta t^{i, j}=0\right)$. In diesem Fall gilt für den sekundären Bjerkneskraftkoeffizieten (4.7a) sowie für die sekundäre Bjerkneskraft (4.7b) offenbar:

$$
f_{\mathrm{Bs}}^{i \rightarrow j}=-f_{\mathrm{Bs}}^{j \rightarrow i} \Rightarrow \vec{F}_{\mathrm{Bs}}^{i \rightarrow j}=-\vec{F}_{\mathrm{Bs}}^{j \rightarrow i}
$$

Die sekundäre Bjerkneskraft wird in Abbildung 4.12 als Wechselwirkung einer im Druckbauch eines Stehwellenfeldes der (1, 1, 1)-Mode positionierten Blase mit einer im Abstand variierten zweiten Blase betrachtet.

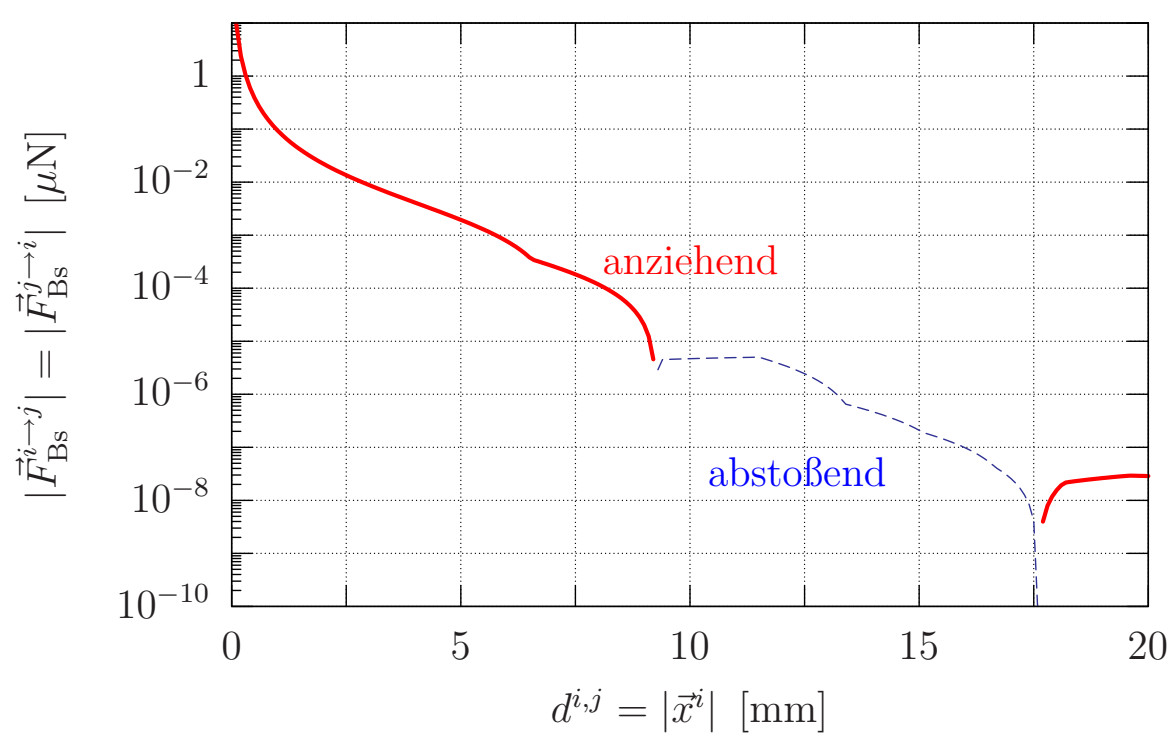

Abbildung 4.12: Beispiel für anziehende (rot) und abstoßende (blau) Bereiche der sekundären Bjerkneskraft abhängig vom Blasenabstand: eine Blase ruht im Druckbauch einer Stehwelle. In der logarithmischen Auftragung sind negative (abstoßende) Bereiche gestrichelt dargestellt; $p_{\mathrm{A}}^{j}=\hat{p}_{\mathrm{A}}=120 \mathrm{kPa}, R_{\mathrm{n}}^{i}=R_{\mathrm{n}}^{j}=7 \mu \mathrm{m}, \nu=20 \mathrm{kHz}, \Delta t^{i, j}=0, \eta=1$ (isotherm). 
Wie beispielsweise in Auftragung 4.12 zu sehen divergiert die sekundäre Bjerkneskraft im Nahbereich:

$$
\left|\vec{F}_{\mathrm{Bs}}^{i \rightarrow j}\right| \sim \frac{1}{\left|\overrightarrow{d^{i} \rightarrow j}\right|^{2}} \rightarrow \infty \quad \text { für } \quad\left|\vec{d}^{i \rightarrow j}\right| \rightarrow 0
$$

In bestimmten Abständen kommt es durch die unterschiedlichen Blasenschwingungen der an unterschiedlichen Orten des Stehwellenfeldes befindlichen Blasen zu einer Vorzeichenumkehr und somit zu einer Abstoßung der beiden Blasen voneinander. Die Abbildungen 4.13 und 4.14 zeigen die Gebiete in der Parameterebene der maximalen Schalldruckamplitude gegen den Blasenabstand. In den Auftragungen 4.13 wird dabei der Ruheradius der im Druckbauch ruhenden Blase bei $7 \mu \mathrm{m}$ festgehalten und die sich im Abstand $d^{i, j}$ befindliche Blase mit verschiedenen Ruheradien betrachtet.

In den Darstellungen 4.14 wird umgekehrt die Wechselwirkung für eine im Zentrum anwachsende Blase betrachtet, der sich eine Blase mit konstantem Ruheradius von $5 \mu \mathrm{m}$ annähert.

In beiden mittleren Abbildungen von 4.13 und 4.14 ist der Fall einer Blase im Druckbauch $R_{\mathrm{n}}^{j}=7 \mu \mathrm{m}$ und einer bewegten Blase $R_{\mathrm{n}}^{i}=5 \mu \mathrm{m}$ gezeigt. In dieser Konfiguration bildet sich um die maximale Schalldruckamplitude von $\hat{p}_{\mathrm{A}}=100 \mathrm{kPa}$ ein schmaler abstoßender Bereich aus, der bis hin zu kleinsten Blasenabständen wirksam ist. Allerdings übt die sekundäre Bjerkneskraft im Großteil des Parameterraumes, in dem sie deutlichen Einfluss auf die Translationsdynamik nimmt (das heißt bei Blasenabständen kleiner als $3 \ldots 5 \mathrm{~mm}$ ), eine die Blasen einander anziehende Wirkung aus.

Abbildung 4.15 (oben) zeigt die Abhängigkeit der sekundären Bjerkneskraft von der Schalldruckamplitude bei konstanten Ruheradien. Es ist eine Zunahme um mehrere Größenordnungen beim Erhöhen der Druckamplitude von 120 auf $200 \mathrm{kPa}$ zu sehen. Die abstoßenden Kräfte bleiben allerdings auch dann in der Regel vernachlässigbar $\left(<10^{-3} \mu \mathrm{N}\right)$.

Eine Änderung der Größe der im Druckbauch ruhenden Blase $\left(R_{\mathrm{n}}^{j}=5,7,20 \mu \mathrm{m}\right)$ nimmt kaum Einfluss auf die Größenordnung der sekundären Bjerkneskraft wie in Abbildung 4.15 (unten) zu sehen.

Bei Betrachtung einer kompressiblen Flüssigkeit tritt der zeitliche Versatz auf $\left(\Delta t^{i, j} \neq 0\right)$. In ihn geht zum Einen die Laufzeit zwischen den Blasen ein:

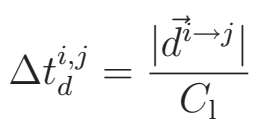

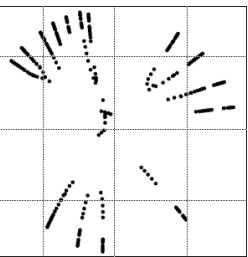




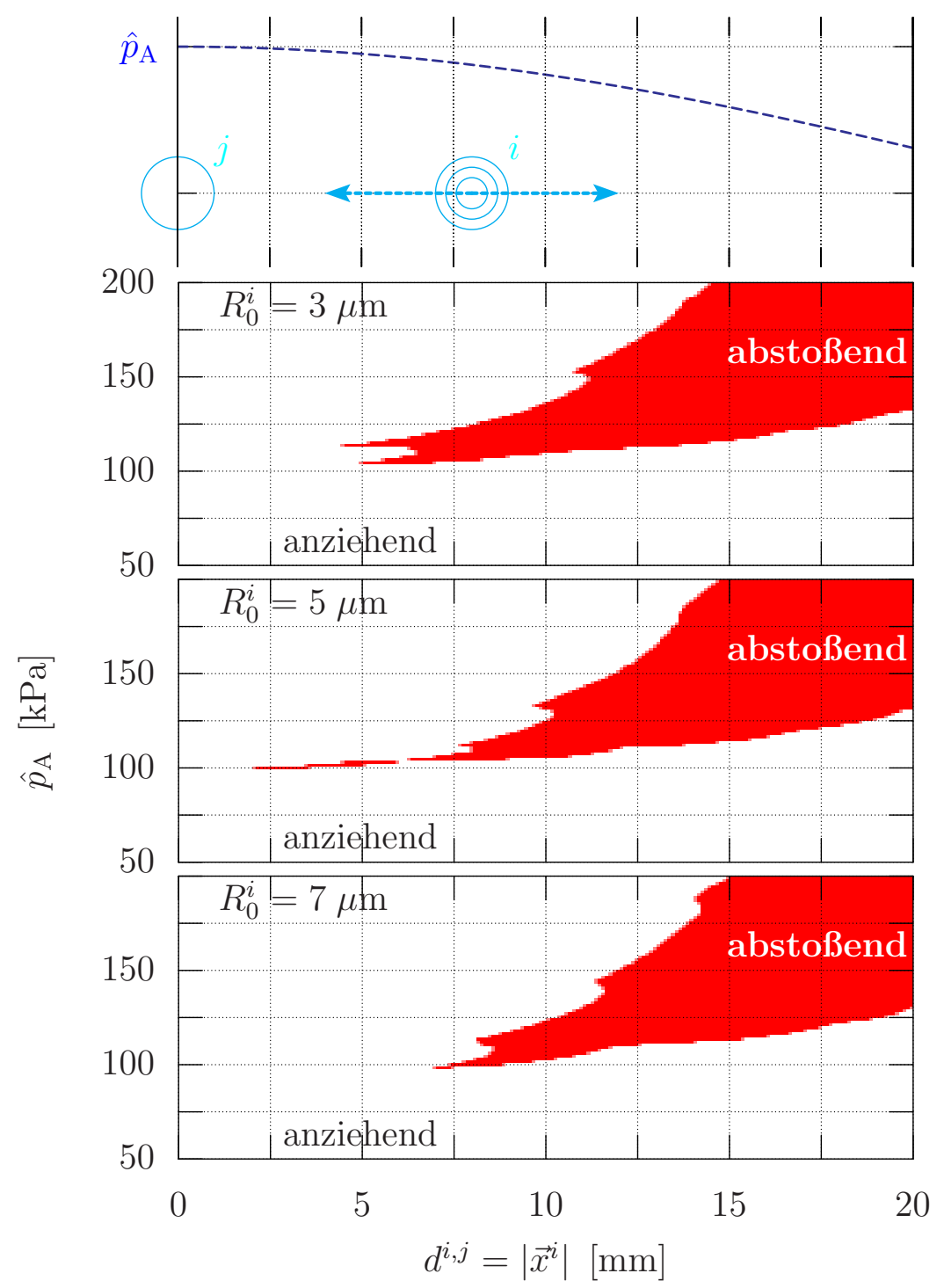

Abbildung 4.13: Vorzeichenwechsel der sekundären Bjerkneskraft zwischen einer Blase im Druckbauch eines Stehwellenfeldes mit konstantem Ruheradius $R_{\mathrm{n}}^{j}=7 \mu \mathrm{m}$ und einer in ihrer Position variierten Blase unterschiedlicher Ruheradien $R_{\mathrm{n}}^{i}=3,5,7 \mu \mathrm{m}$ in der Parameterebene von maximaler Schalldruckamplitude und Blasenabstand. Die variierten Blasenkonfigurationen im Schalldruckverlauf sind in der oberen Skizze verdeutlicht; $\nu=20 \mathrm{kHz}, \eta=1, \Delta t^{i, j}=0$.

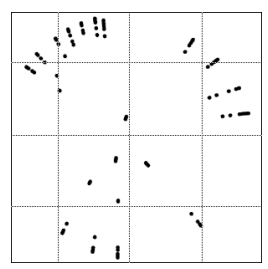




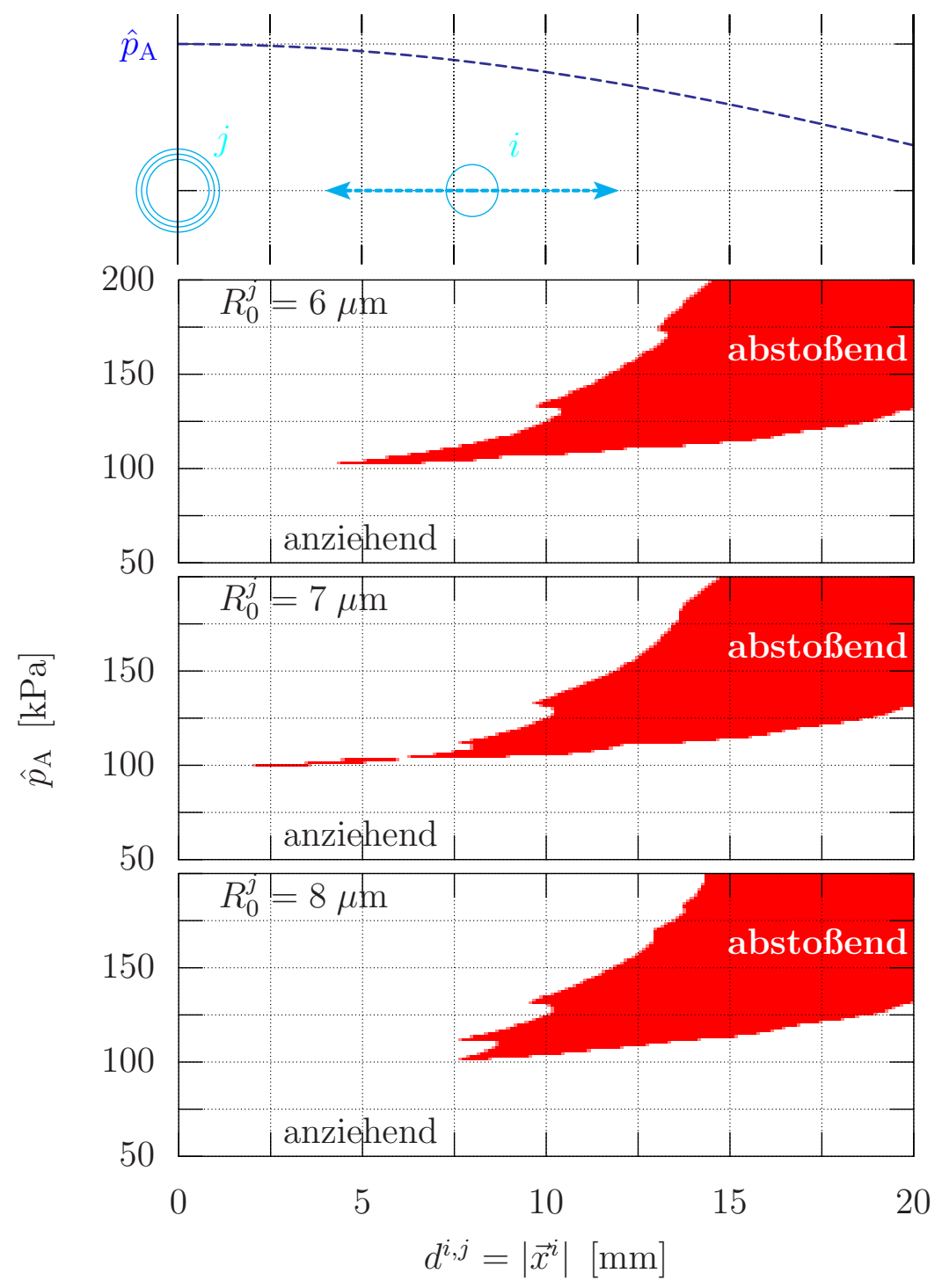

Abbildung 4.14: Vorzeichenwechsel der sekundären Bjerkneskraft zwischen einer Blase im Druckbauch eines Stehwellenfeldes für variierten Ruheradius $R_{\mathrm{n}}^{j}=6,7,8 \mu \mathrm{m}$ und einer in ihrer Position variierten Blase mit konstantem Ruheradius $R_{\mathrm{n}}^{i}=5 \mu \mathrm{m}$ in der Parameterebene von maximaler Schalldruckamplitude und Blasenabstand. Die variierten Blasenkonfigurationen im Schalldruckverlauf sind in der oberen Skizze verdeutlicht; $\nu=20 \mathrm{kHz}, \eta=1, \Delta t^{i, j}=0$.

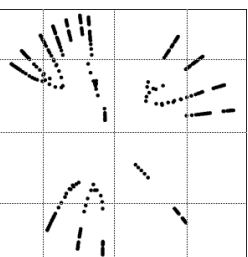




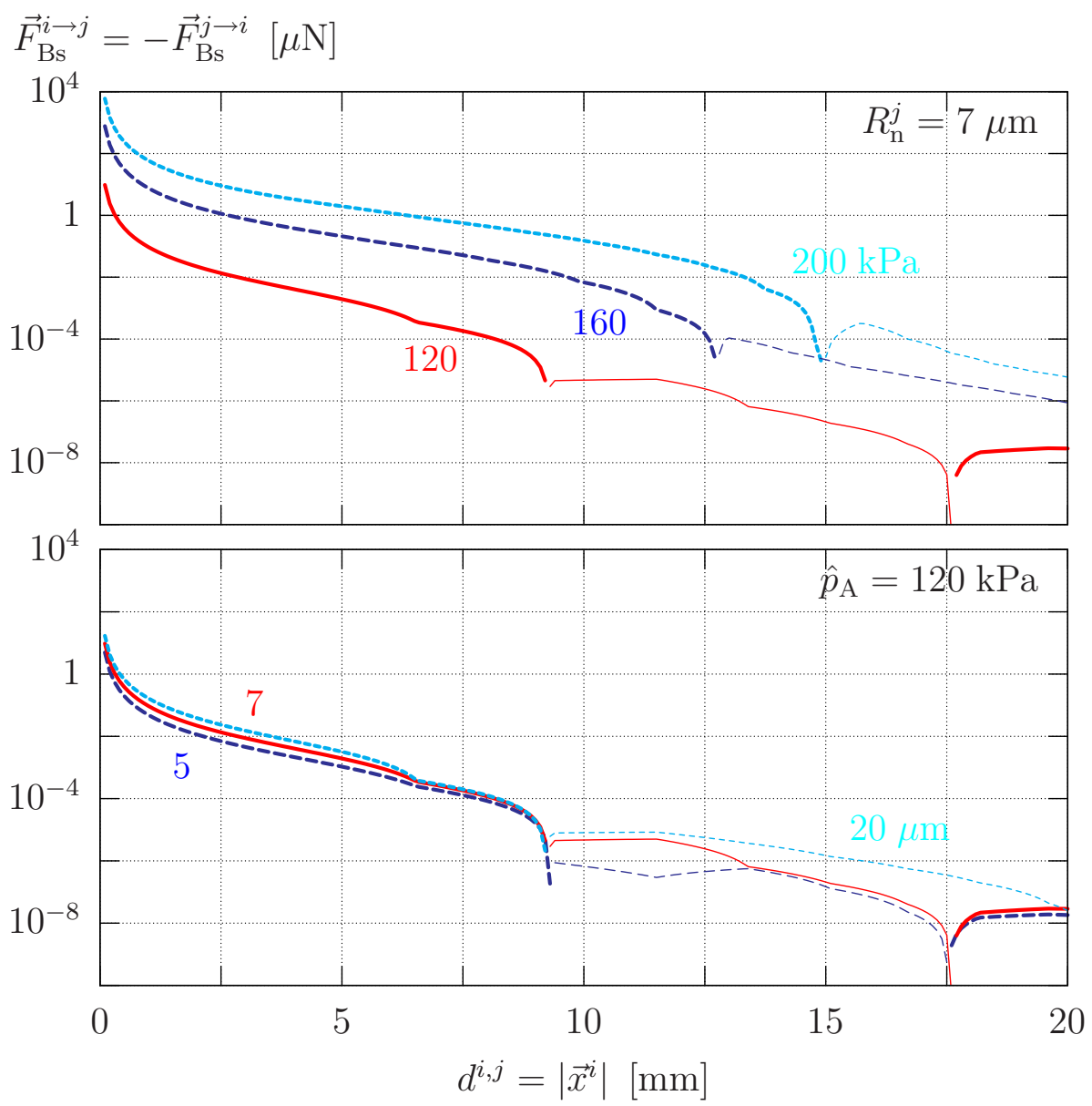

Abbildung 4.15: Beeinflussung der sekundären Bjerkneskraft durch die Schalldruckamplitude $\hat{p}_{\mathrm{A}}=120,160,200 \mathrm{kPa}$ bei konstantem Ruheradius $R_{\mathrm{n}}^{j}=7 \mu \mathrm{m}$ (oben) und durch verschiedene Ruheradien $R_{\mathrm{n}}^{j}=5,7,20 \mu \mathrm{m}$ der im Druckbauch $\left(\hat{p}_{\mathrm{A}}=120 \mathrm{kPa}\right)$ ruhenden Blase (unten). Negative (abstoßende) Bereiche sind durch dünnere Linien gekennzeichnet; $R_{\mathrm{n}}^{i}=$ $7 \mu \mathrm{m}, \nu=20 \mathrm{kHz}, \Delta t^{i, j}=0, \eta=1$.

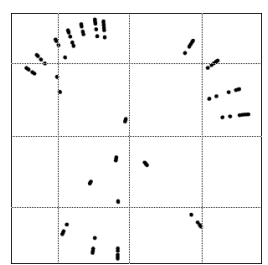


Die sekundäre Bjerkneskraft wird im Allgemeinen von der deutlich stärkeren primären Bjerkneskraft überlagert und tritt erst ab Blasenabständen kleiner etwa $3 \mathrm{~mm}$ in Erscheinung. Die daraus resultierende Laufzeit beträgt:

$$
\Delta t_{d}^{i, j}=\frac{\left|\overrightarrow{d^{i} \rightarrow j}\right|}{C_{1}} \approx 2 \mu \mathrm{s}
$$

Außerdem kann sofern vorhanden eine Phasendifferenz zwischen den Schalldrücken an den Blasenpositionen als zeitlicher Versatz aufgefasst werden:

$$
\begin{aligned}
\Delta \varphi^{i \rightarrow j} & =\varphi^{j}-\varphi^{i} \Rightarrow \Delta t_{\varphi}^{i, j}=\frac{\tau_{\mathrm{osz}}}{2 \pi} \Delta \varphi^{i \rightarrow j} \\
& \Rightarrow \quad \Delta t^{i, j}=\Delta t_{d}^{i, j}+\Delta t_{\varphi}^{i, j}
\end{aligned}
$$

In einem Stehwellenfeld werden aufgrund der an allen Blasenpositionen gleichphasigen Oszillation des Schallfeldes auch die Blasen gleichphasig getrieben $\left(\Delta t_{\dot{\varphi}}^{i, j}=0\right)$. So kommt es nur durch die Verzögerung in der Laufzeit zu einer Verschiebung der radialen Blasenpulsationen gegeneinander:

$$
\Delta t^{i, j}=\Delta t_{d}^{i, j}
$$

Die Auswirkung dieser Verschiebung ist beispielhaft in Abbildung $4.16 \mathrm{zu}$ sehen. Dieser Phasenversatz nimmt bei der sehr schwachen sekundären Bjerkneskraft keinen Einfluss auf die weiteren Rechnungen und kann vernachlässigt werden. In dem spitzen Einbruch der sekundären Bjerkneskraft bei $\hat{p}_{\mathrm{A}}=100 \mathrm{kPa}$ wechselt das Vorzeichen für diesen Druckwert, so dass es zu einer Abstoßung zwischen den Blasen kommt.

Unter Vernachlässigung der Laufzeit gilt immer:

$$
\vec{F}_{\mathrm{Bs}}^{i \rightarrow j}=-\vec{F}_{\mathrm{Bs}}^{j \rightarrow i} \quad \text { bei } \quad \Delta t^{i, j}=0
$$

Bei Einbeziehung der Phasenverzögerung zwischen den wechselwirkenden Blasen gilt diese Gleichheit nur in dem Spezialfall identischer Blasenpulsationen, das heißt gleichen Ruheradien und übereinstimmender Schalldruckamplituden:

$$
\vec{F}_{\mathrm{Bs}}^{i \rightarrow j}=-\vec{F}_{\mathrm{Bs}}^{j \rightarrow i} \quad \text { bei } \quad \Delta t^{i, j} \neq 0 \quad \text { gilt für } \quad R_{\mathrm{n}}^{i}=R_{\mathrm{n}}^{j} \quad \text { und } \quad p_{\mathrm{A}}^{i}=p_{\mathrm{A}}^{j}
$$

In Abbildung 4.17 sind die unterschiedlichen sekundären Bjerkneskräfte für die Wirkung von Blase $i$ auf Blase $j$ und in umgekehrter Richtung von Blase $j$ auf Blase $i$ aufgetragen. Zusätzlich ist die Kraft unter Vernachlässigung des Laufzeiteffektes eingezeichnet. Die sich abzeichnenden Abweichungen in der Kraft treten überwiegend bei Werten $<10^{-3} \mu \mathrm{N}$ auf und lassen sich gegenüber der ebenfalls wirkenden in Abschnitt 4.2 betrachteten primären Bjerkneskraft vernachlässigen, so dass im Folgenden die Laufzeit nicht mehr betrachtet wird $\left(\Delta t^{i, j}=0\right)$.

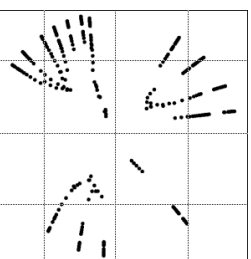




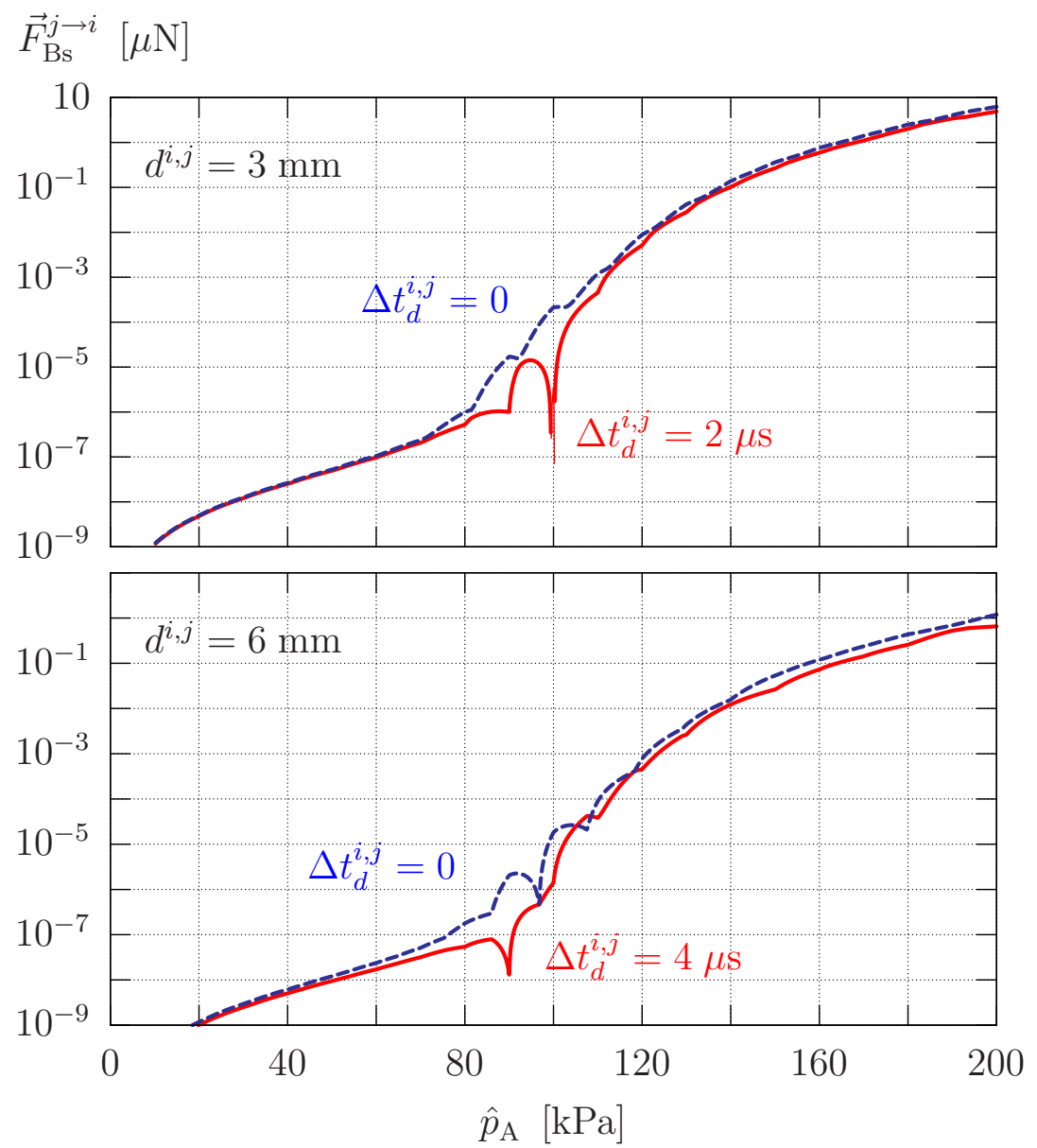

Abbildung 4.16: Auswirkung der laufzeitbedingten Phasenverschiebung auf die sekundäre Bjerkneskraft. Blase $j$ ruht im Druckbauch; $d^{i, j}=3 \mathrm{~mm}$ (oben) und $6 \mathrm{~mm}$ (unten), $R_{\mathrm{n}}^{i}=R_{\mathrm{n}}^{j}=7 \mu \mathrm{m}, p_{\mathrm{A}}^{j}=\hat{p}_{\mathrm{A}}, \nu=20 \mathrm{kHz}, \eta=1$ (isotherm), $\Delta t_{\varphi}^{i, j}=0$ (Stehwellenfeld).

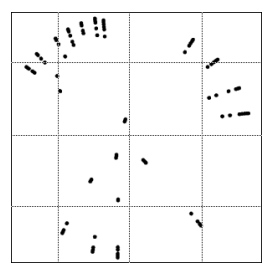




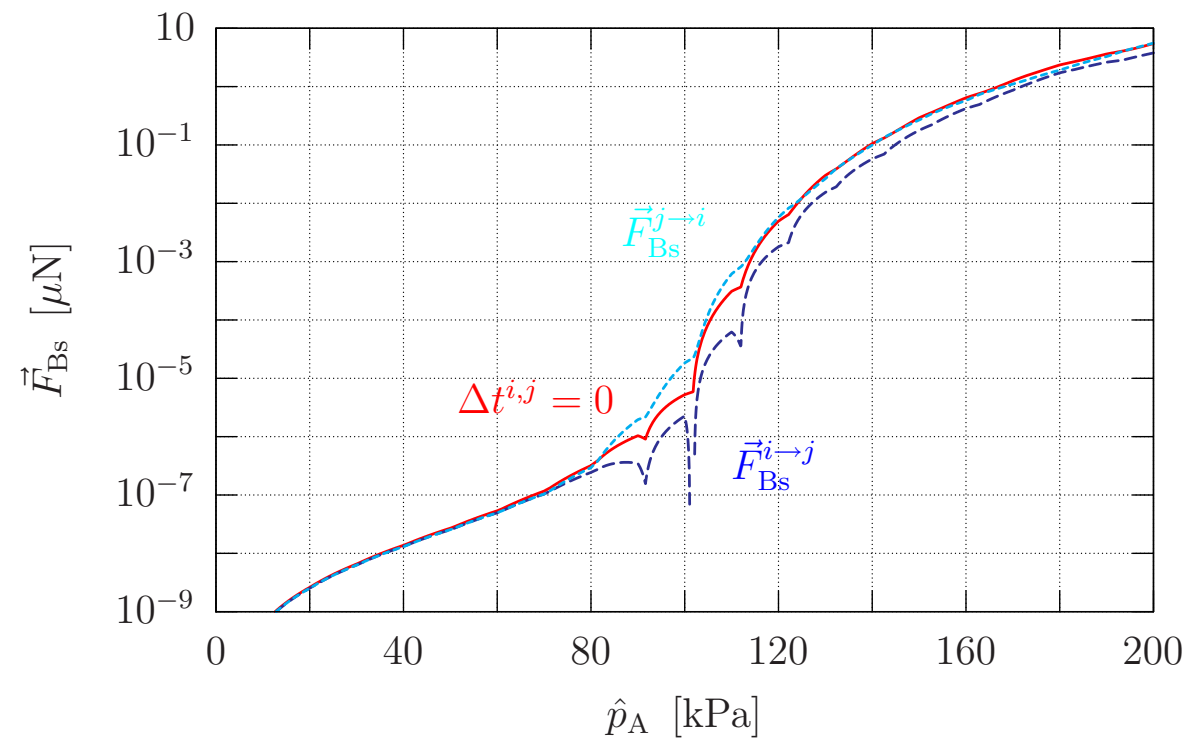

Abbildung 4.17: Unterscheidung der sekundären Bjerkneskraft von Blase $i$ auf Blase $j$ und von Blase $j$ auf Blase $i$. Blase $j$ befindet sich im Druckbauch eines radialsymmetrischen Schallfeldes; $d^{i, j}=3 \mathrm{~mm}, p_{\mathrm{A}}^{j}=\hat{p}_{\mathrm{A}}$, $R_{\mathrm{n}}^{j}=8 \mu \mathrm{m}, R_{\mathrm{n}}^{i}=5 \mu \mathrm{m}, \nu=20 \mathrm{kHz}, \eta=1$ (isotherm).

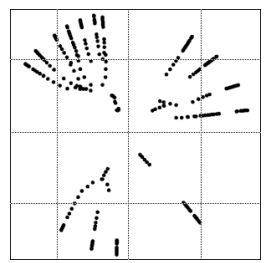




\subsection{Reibungskraft}

Die viskose Reibungskraft ist der Bewegung einer Blase entgegengerichtet und begrenzt ihre Translationsgeschwindigkeit. Für niedrige Geschwindigkeiten $(|\vec{v}|<$ $0.1 \mathrm{~m} / \mathrm{s})$ und kleine Objekte $(R \lesssim 10 \mu \mathrm{m})$ scheint zunächst die Annahme einer laminaren, schleichenden Strömung gerechtfertigt. Die Reibung wird hier als proportional $^{7}$ zu der Geschwindigkeit der Blase und ihrer Querschnittsfläche senkrecht zur Bewegungsrichtung angenommen:

$$
\vec{F}_{\mathrm{D}}=\vec{F}_{\mathrm{D}}(\vec{v}, R) \sim-\vec{v} \pi R^{2}
$$

Exakt müsste mit der Relativgeschwindigkeit $\vec{w}$ der Geschwindigkeit des Blasenschwerpunktes $\vec{v}$ gegenüber der bewegten Flüssigkeit $\vec{u}$ gerechnet werden, wie es Abbildung 4.18 zeigt: $\vec{w}=\vec{v}-\vec{u}$. In den betrachteten Resonatoren wird die Flüssigkeitsströmung ${ }^{8}$ vernachlässigt: $\vec{w}=\vec{v}$. Außerdem wird bereits eine über die Pulsationsperiode gemittelte Geschwindigkeit angenommen: $\vec{v} \equiv\langle\vec{v}\rangle_{\tau_{\text {osz }}}$

In der Literatur sind unterschiedliche Reibungsmodelle zu finden, die verschiedene Fälle beschreiben. Die in Abbildung 4.18 skizzierte Unterscheidung in die „no slip“ und „slip“ Bedingung entsprechen einer harten Kugel, an deren Oberfläche die Tangentialkomponente der Strömung auf Null abfällt, bzw. einer Gasblase, deren Moleküle entsprechend der Flüssigkeitsströmung zirkulieren.

Als charakteristische Größe für den viskosen Widerstand dient die beispielsweise von Gerthsen et al. [76] angegebene Reynolds-Zahl:

$$
R e=\frac{2 \varrho_{1} R|\vec{v}|}{\mu}
$$

$\varrho_{1} \quad$ Dichte des Fluids

$\mu$ dynamische Viskosität

Die Annahme einer harten Kugel ist eine Näherung, die bei an der Blase anlagernden Verschmutzungen gemacht werden kann. CRUM und ElLER [78] geben die entsprechende Stokes'sche Reibung an:

$$
\vec{F}_{\mathrm{D}}=-6 \pi \mu R \vec{v}
$$

7 In einer turbulenten Strömung würde nach GERTHSEN et al. [76] die Newton'sche Reibung $\vec{F}_{\mathrm{D}} \sim \vec{v}^{2}$ gelten.

8 Genaugenommen müsste hier die durch das Schallfeld verursachte Schallschnelle in der Flüssigkeit betrachtet werden, wie sie beispielsweise DoINIKOV [77] zur Untersuchung einer Einzelblase im Stehwellenfeld einsetzt. Dies wird hier aber ebenfalls vernachlässigt. 

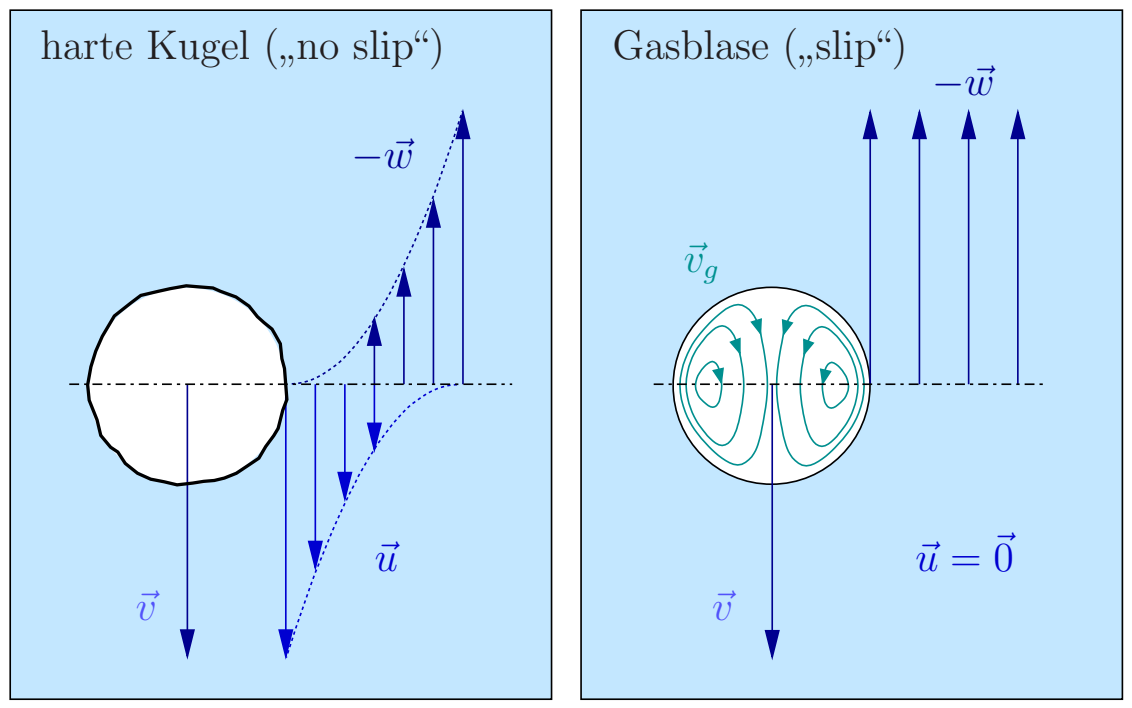

Abbildung 4.18: Darstellung des laminaren Strömungsprofils bei einer durch eine Flüssigkeit laufenden Blase. Links: Die angrenzende Flüssigkeitsschicht haftet an der Schmutz behafteten Blasenoberfläche $(\vec{u}=\vec{v})$, mit zunehmender Entfernung geht die Strömungsgeschwindigkeit gegen Null $(\vec{u} \rightarrow \overrightarrow{0})$. Rechts: Durch seine Zirkulation bietet das Gas in der Blase keinen Angriffspunkt für die angrenzende Flüssigkeitsschicht, das heißt die Blase „gleitet" ohne die Flüssigkeit mitzunehmen.

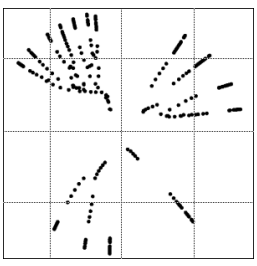


Wie es beispielsweise bei BATChelor [67] zu finden ist, ist es in der Literatur üblich, einen dimensionslosen Reibungskoeffizienten einzuführen, der im Zusammenhang mit der Reynolds-Zahl steht. Für die Stokes'sche Reibung (4.9), die linear mit $|\vec{v}|$ wächst, lässt sich der Koeffizient angeben als:

$$
C_{\mathrm{D}} \equiv \frac{2\left|\vec{F}_{\mathrm{D}}\right|}{\varrho_{1}|\vec{v}|^{2} \pi R^{2}}=\frac{4 \alpha}{R e}
$$

Für die Stokes'sche Reibung einer harten Kugel ergibt sich so ein Faktor von $\alpha=$ 6. Die Reibungskraft schreibt sich unter Verwendung des Reibungskoeffizienten in der Form:

$$
\vec{F}_{\mathrm{D}}=-C_{\mathrm{D}} \frac{\varrho_{1}}{2} \pi R^{2}|\vec{v}| \vec{v}=-\alpha \mu \pi R \vec{v}
$$

Für eine oszillierende leere Blase sind die Fälle kleiner und großer Reynolds-Zahl zu unterscheiden. Der Fall kleiner Reynolds-Zahl entspricht der von LANDAU und Lifschitz [69] angegebenen Stokes'schen Reibung einer leeren Blase mit $\alpha=4$. Die Unterscheidung der Fälle $R e \gg 1$ und $R e \ll 1$ werden beispielsweise von Magnaudet und Legendre [79] oder von RedDy und Szeri [80] diskutiert:

$$
\begin{array}{llll}
\vec{F}_{\mathrm{D}}=-4 \pi \mu R \vec{v} & \text { für } & R e \ll 1 & \text { (laminar) } \\
\vec{F}_{\mathrm{D}}=-12 \pi \mu R \vec{v} & \text { für } & R e \gg 1 & \text { (turbulent) }
\end{array}
$$

Eine symmetrische Strömung bei sehr kleiner Reynolds-Zahl Re $<1$ verliert mit steigender Reynolds-Zahl ihre Symmetrie, nach TRITTON [68] kann sie bis etwa $R e \approx 40$ als laminar eingestuft werden, bevor sie für hohe Reynolds-Zahlen turbulent wird. Typische Reynolds-Zahlen liegen für Blasen mit einem mittleren Radius von $R=10 \mu \mathrm{m}$ und einer Geschwindigkeit von $|\vec{v}|=0.1 \mathrm{~m} / \mathrm{s}$ bei $R e \approx$ 2. Stark beschleunigte Blasen können sich kurzzeitig auch in Regimen höherer Reynolds-Zahlen bewegen.

Ausgehend von einer linearisierten Bewegungsgleichung wird von KREFTing [19] eine Anpassung des Vorfaktors $\alpha$ an experimentelle Daten vorgenommen. In Anlehnung an frühere Arbeiten von CRUM und ElLER [78] wird eine Blase zunächst im Druckbauch eines Stehwellenfeldes der $(1,1,1)$-Mode in der bereits in Abschnitt 3.2 vorgestellten Hellma-Küvette „gefangen“. Nach Absenken der Schalldruckamplitude beginnt die Blase aufzusteigen, wobei sie weiterhin pulsiert.

Die Bewegungsgleichung zur numerischen Beschreibung beinhaltet die virtuelle Masse, die primäre Bjerkneskraft, die Reibungskraft sowie den Auftrieb.

Der Auftrieb ${ }^{9}$ einer Blase bestimmt sich aus der Masse der verdrängten Flüs-

9 In einem starken Schallfeld kann der Auftrieb gegen die primäre Bjerkneskraft vernachlässigt werden. Lediglich bei geringem Schalldruck und nahe einem Druckknoten kommt der Auftrieb zum Tragen. 
sigkeit multipliziert mit der Gravitationskonstanten:

$$
\vec{F}_{\text {auf }}=\varrho_{\mathrm{l}} \frac{4}{3} \pi R^{3} \cdot \vec{g}_{\mathrm{z}}
$$

Abbildung 4.19 zeigt die experimentellen Daten von zwei Messungen im Vergleich zu Lösungen der Bewegungsgleichung mit den entsprechenden Reibungskräften.

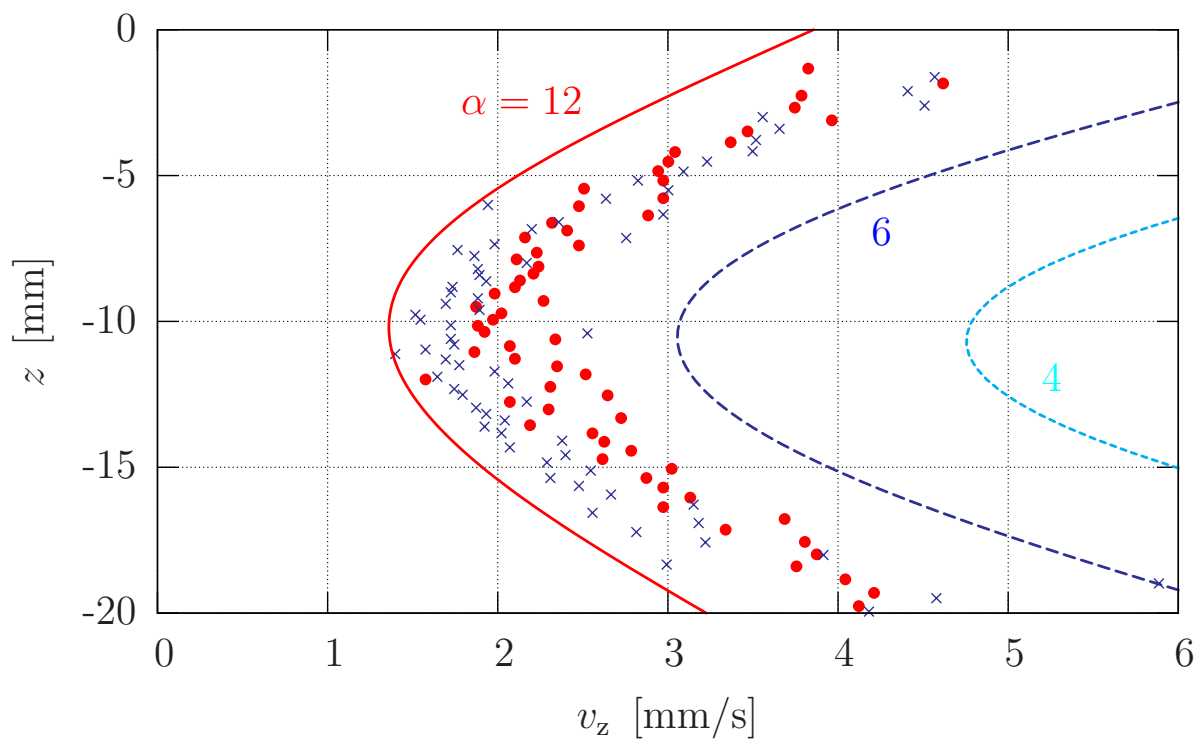

Abbildung 4.19: Experimentell bestimmte Aufstiegsgeschwindigkeiten pulsierender Blasen verglichen mit theoretischen Beschreibungen der Reibungskraft (farbige Linien). Die gepunkteten Verläufe stellen zwei experimentelle Verläufe dar (übernommen von KREFTiNG [19]); $p_{\mathrm{A}} \approx 5.3 \mathrm{kPa}$, $R_{\mathrm{n}} \approx 58 \mu \mathrm{m}$.

Die von dem Druckbauch $(z \approx-20 \mathrm{~mm})$ aus startenden Blasen werden durch den Auftrieb zur Wasseroberfläche $(z=0)$ hin beschleunigt. Gleichzeitig bremst sie die primäre Bjerkneskraft ab. Auf halbem Weg zwischen Druckbauch und Druckknoten $(x \approx-10 \mathrm{~mm})$ ist ein Minimum der Geschwindigkeit bzw. ein Maximum der primären Bjerkneskraft zu sehen. Dieser Effekt erklärt sich durch Betrachtung des linearisierten Systems. Dabei ist die Blasenauslenkung direkt proportional zum Schalldruck multipliziert mit dem Schalldruckgradienten und liefert genau das beobachtete Maximum der primären Bjerkneskraft.

Die von Krefting [19] beobachteten Blasen liegen in ihrer Aufstiegsgeschwindigkeit zwischen dem Modell einer leeren pulsierenden Blase bei hoher Reynolds-Zahl $(\alpha=12)$ und dem einer harten Kugel $(\alpha=6)$. Der vorgeschlagene Faktor liegt bei $\alpha=11$ und führt auf die Reibungskraft:

$$
\vec{F}_{\mathrm{D}}=-11 \pi \mu R \vec{v}
$$

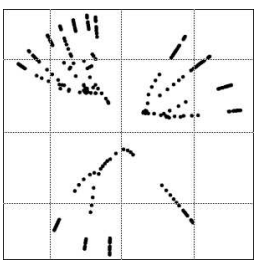




\subsection{Partikelmodell}

Die Partikelmodellierung löst die gekoppelten Bewegungsgleichungen eines $N$ Blasensystems $(i=1 \ldots N)$. Die Newton'schen Bewegungsgleichungen beschreiben die Kraftwirkungen auf jede einzelne Blase:

$$
\vec{F}_{\mathrm{M}}^{i}+\vec{F}_{\mathrm{Bp}}^{i}+\vec{F}_{\mathrm{Bs}}^{i}+\vec{F}_{\mathrm{D}}^{i}=\overrightarrow{0}
$$

Die Kopplungen dieser Gleichungen bestehen über die Wechselwirkung der Blasen beschrieben durch die sekundäre Bjerkneskraft sowie über eine eventuelle Rückwirkung der Blasen auf das Schallfeld, von dem sie getrieben werden.

Für die direkte Integration der Bewegungsgleichungen werden die virtuelle Masse (4.1), die primäre Bjerkneskraft (4.4), die sekundäre Bjerkneskraft (4.5) und die Reibungskraft (4.10) herangezogen:

$$
\begin{aligned}
\vec{F}_{\mathrm{M}}^{i} & =\frac{\varrho_{\mathrm{l}}}{2} V^{i} \ddot{\vec{x}}^{i} \\
\vec{F}_{\mathrm{Bp}}^{i} & =-\nabla \tilde{p}\left(\vec{x}^{i}\right) V^{i} \\
\vec{F}_{\mathrm{Bs}}^{i} & =\sum_{j \neq i} \vec{F}_{\mathrm{Bs}}^{j \rightarrow i} \\
\vec{F}_{\mathrm{Bs}}^{j \rightarrow i} & =\frac{\varrho_{\mathrm{l}}}{4 \pi}\left\langle\dot{V}^{j}(t) \dot{V}^{i}\left(t-\Delta t^{i, j}\right)\right\rangle_{\tau_{\mathrm{zyk}}^{\mathrm{i}, \mathrm{j}}} \frac{\vec{d}^{i \rightarrow j}}{\left|\vec{d}^{i \rightarrow j}\right|^{3}} \\
\vec{F}_{\mathrm{D}}^{i} & =-\alpha \pi \mu R^{i} \dot{\vec{x}}^{i}
\end{aligned}
$$

Zusammen ergeben diese Kräfte die Formulierung der Bewegungsgleichung (4.13) in der Form:

$$
\begin{aligned}
\vec{F}_{\mathrm{M}}^{i} & =-\vec{F}_{\mathrm{D}}^{i}-\vec{F}_{\mathrm{Bp}}^{i}-\vec{F}_{\mathrm{Bs}}^{i} \\
\Leftrightarrow \frac{\varrho_{\mathrm{l}}}{2} V^{i}(t) \ddot{\vec{x}}^{i} & =\alpha \pi \mu R^{i}(t) \dot{\vec{x}}^{i}+\nabla \tilde{p}\left(\vec{x}^{i}, t\right) V^{i}(t)-\sum_{j \neq i} \vec{F}_{\mathrm{Bs}}^{j \rightarrow i}
\end{aligned}
$$

Die Lösung dieser Differentialgleichungen unter den Anfangsbedingungen $\vec{x}^{i}(t=$ $0)=\vec{x}_{0}^{i}$ liefert einen kontinuierlichen Verlauf der Blasentrajektorie:

$$
\vec{x}^{i}(t), \quad t \in \mathbb{R}^{+}
$$

Der Gradient des akustischen Wechseldrucks leitet sich ab aus der Formulierung des Wechseldrucks (3.2) zu

$$
\nabla \tilde{p}=\nabla p_{\mathrm{A}} \sin (\omega t-\varphi)-p_{\mathrm{A}} \nabla \varphi \cos (\omega t-\varphi)
$$


Die Blasendynamik $R(t)$ wird durch das Keller-Miksis-Modell (2.10) beschrieben.

Die Betrachtung der kompletten Bewegungsgleichungen bei einem Vielblasensystem und dessen Aufintegration bedeutet einen erheblichen Rechenaufwand, zumal sich die Zeitskalen des Problems über einen großen Bereich erstrecken. Die Partikelmodellierung soll die Dynamik des Gesamtsystems auf SekundenZeitskalen wiedergeben. Eine Integration der Bewegungsgleichungen (4.14) wird beispielsweise von LUTHER [81] ausgeführt.

Die unterschiedlichen Zeitskalen der vorgestellten Dynamiken gestatten eine entkoppelte Behandlung der Blasenoszillation, der Translation sowie der Ausbildung komplexer Blasenstrukturen:

$$
\tau_{\text {osz }}[\mu \mathrm{s}] \ll \tau_{\text {trans }}[\mathrm{ms}] \ll \tau_{\text {stream }}[\mathrm{s}]
$$

1. Im Bereich der Mikrosekunden-Skala liegt die Schallfeldperiode, und es läuft die aus Kapitel 2 bekannte radiale Blasenpulsation ab.

2. Die in diesem Kapitel vorgestellte Blasentranslation läuft auf der Millisekunden-Skala ab.

3. Änderungen der in Kapitel 3 betrachteten räumlichen Schallfelddynamik laufen auf der Sekunden-Skala ab, ebenso die Ausbildung der im folgenden Kapitel betrachteten Streamerfiguren.

Da die translatorische Dynamik der Blasen, also die Lösungen der Bewegungsgleichungen auf einer langsameren Zeitskala ablaufen gegenüber der Oszillationsdynamik, können diese Dynamiken getrennt betrachtet werden. Dies geschieht durch die Mittelung der Bewegungsgleichungen (4.14) über eine Schallfeldperiode:

$$
\begin{aligned}
\frac{\varrho_{1}}{2}\left\langle V^{i}\right\rangle_{\tau_{\mathrm{osz}}} \ddot{\vec{x}}^{i}= & \alpha \pi \mu\left\langle R^{i}\right\rangle_{\tau_{\mathrm{osz}}} \dot{\vec{x}}^{i} \\
& +\nabla p_{\mathrm{A}}\left\langle V^{i} \sin (\omega t)\right\rangle_{\tau_{\mathrm{osz}}} \\
& -p_{\mathrm{A}} \nabla \varphi\left\langle V^{i} \cos (\omega t)\right\rangle_{\tau_{\mathrm{osz}}} \\
& -\sum_{j \neq i} \frac{\varrho_{1}}{4 \pi}\left\langle\dot{V}^{j}(t) \dot{V}^{i}\left(t-\Delta t^{i, j}\right)\right\rangle_{\tau_{\mathrm{zyk}}^{\mathrm{i}, j}} \frac{\vec{d}^{i \rightarrow j}}{\left|\vec{d}^{i \rightarrow j}\right|^{3}}
\end{aligned}
$$

Die Lösungen dieser gemittelten Bewegungsgleichungen ergeben diskretisierte Blasentrajektorien:

$$
\vec{x}^{i}(t), \quad t=n \tau_{\text {osz }}, \quad n=0,1,2, \ldots
$$

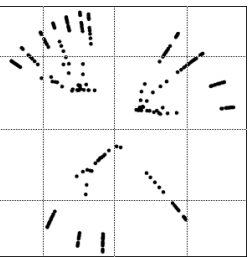


Durch die Mittelung gehen jedoch Effekte verloren. So wird etwa die ruckartige Bewegung der Blase nicht mehr aufgelöst. Diese rührt daher, dass die in der Expansionsphase größer aufgezogene Blase stärker abgebremst wird und sich langsamer bewegt als die kleinere komprimierte Blase, die stärker beschleunigt wird, wodurch es während einer Oszillationsperiode der Blase zu Variationen in der Geschwindigkeit kommt.

Mit der Mittelung der Bewegungsgleichungen bietet es sich an, eine Koeffizientendarstellung zu wählen:

$$
\begin{aligned}
f_{\mathrm{M}}^{i} & =\frac{\varrho_{\mathrm{l}}}{2}\left\langle V^{i}\right\rangle_{\tau_{\mathrm{osz}}} \\
f_{\mathrm{D}}^{i} & =\pi \mu\left\langle R^{i}\right\rangle_{\tau_{\mathrm{osz}}} \\
f_{\mathrm{Bp}}^{\nabla p_{\mathrm{A}}{ }^{i}} & =\left\langle V^{i} \sin (\omega t)\right\rangle_{\tau_{\mathrm{osz}}} \\
f_{\mathrm{Bp}}^{\nabla \varphi^{i}} & =\left\langle V^{i} \cos (\omega t)\right\rangle_{\tau_{\mathrm{osz}}} \\
f_{\mathrm{Bs}}^{j \rightarrow i} & =\frac{\varrho_{\mathrm{l}}}{4 \pi}\left\langle\dot{V}^{i}(t) \cdot \dot{V}^{j}\left(t-\Delta t^{i, j}\right)\right\rangle_{\tau_{\mathrm{zyk}}^{\mathrm{i}, \mathrm{j}}}
\end{aligned}
$$

Die Bewegungsgleichungen schreiben sich somit als:

$$
f_{\mathrm{M}}^{i} \ddot{\vec{x}}^{i}=\alpha f_{\mathrm{D}}^{i} \dot{\vec{x}}^{i}+\nabla p_{\mathrm{A}} f_{\mathrm{Bp}}^{\nabla p_{\mathrm{A}}^{i}}-p_{\mathrm{A}} \nabla \varphi f_{\mathrm{Bp}}^{\nabla \varphi^{i}}-\sum_{j \neq i} f_{\mathrm{Bs}}^{j \rightarrow i} \frac{\vec{d}^{i \rightarrow j}}{\left|\vec{d}^{i \rightarrow j}\right|^{3}}
$$

Ein deutlicher Geschwindigkeitsvorteil für die Behandlung von Vielblasensystemen $(N \gtrsim 500)$ wird erreicht, indem die Koeffizienten abhängig von den möglichen auftretenden Druckamplituden und Ruheradien vor der Lösung der Bewegungsgleichung (4.17) tabelliert abgelegt werden.

In diesen Tabellen steckt im Wesentlichen der Rechenaufwand zur Bestimmung der radialen Blasendynamik. Durch die Diskretisierung der Koeffizienten im Parameterraum für Ruheradius und Schalldruckamplitude wird eine Interpolation zwischen den Stützpunkten der gespeicherten Werte notwendig, die im Fall einer zu grobmaschigen Tabellierung einen Fehler verursacht.

\subsubsection{Programmablauf}

Vor dem eigentlichen Ablauf der Partikelmodellierung müssen die über die Schallfeldperiode gemittelten Koeffizienten (4.16a) - (4.16e) in dem zu erwartenden Parameterbereich von Schalldruckamplitude und Ruheradius bestimmt und tabelliert werden.

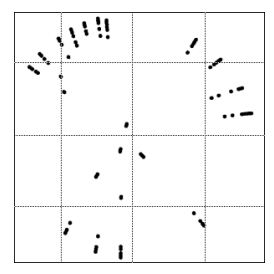


Die Generierung neuer Blasen erfolgt an vorgegebenen Blasenquellen, die ortsfest zu definierten Zeitpunkten neue Blasen in die Simulation einbringen. Verteilt werden diese Quellpunkte in Anlehnung an experimentelle Beobachtungen.

Das Schallfeld bestimmt sich aus der Lösung der modifizierten gedämpften Helmholtz-Gleichung (3.25). In die Bestimmung des Schallfeldes geht die aktuelle Blasenverteilung in dem Resonator ein. Da die Veränderung der Schallfeldverteilung auf einer langsameren Zeitskala abläuft als die Translation der Blasen, werden die Blasenspuren quasi als „Langzeitbelichtung“ zum Lösen der HelmholtzGleichung verwendet.

Mit der Anwesenheit vieler Blasen kommt es zu einer nicht mehr zu vernachlässigenden Rückwirkung der Blasen auf die Struktur des Schallfeldes, das heißt nach einer gewissen Anzahl von Schallfeldperioden ist das Schallfeld neu zu berechnen.

Die Einkopplung der Blasenverteilung in die Schallfeldberechnung erfolgt über eine Blasendichteverteilung auf einem Gitter, die auf eine Änderung der Schallgeschwindigkeit im Fluid abgebildet wird. Durch die Anwesenheit von Blasenwolken wird die Schallgeschwindigkeit deutlich beeinflusst. Dagegen bleibt die Dichte des Zweiphasenmediums relativ unbeeinflusst, da das aufsummierte Blasenvolumen gegenüber dem Fluidvolumen vernachlässigbar gering bleibt.

Als Kriterium einer Blasenkollision wird abgefragt, ob der Abstand der fraglichen Blasen kleiner ist als die Summe der Maximalradien, die sie während einer Integrationsperiode eingenommen haben:

$$
\overrightarrow{d^{c} \rightarrow j} \stackrel{?}{<} R_{\max }^{i}+R_{\max }^{j}, \quad R_{\max }=\max _{\tau_{\mathrm{osz}}}(R(t))
$$

Falls es zu einer Kollision kam, wird eine Verschmelzung der beteiligten Blasen ( $i$ und $j$ ) angenommen und die neue Blase $(k)$ in den Schwerpunkt der zusammenlaufenden Blasen gesetzt:

$$
\vec{x}^{k}=\vec{x}^{i}+\vec{d}^{i \rightarrow j} \frac{V^{j}}{V^{k}}
$$

Die Vereinigung der Blasen erfolgt unter Beachtung der Massenerhaltung des Gases in der Blase:

$$
m^{k}=m^{i}+m^{j} \Rightarrow R_{\mathrm{n}}^{k}=\sqrt[3]{R_{\mathrm{n}}^{i^{3}}+R_{\mathrm{n}}^{j^{3}}}
$$

Die neue Geschwindigkeit der vereinten Blasen folgt aus der Impulserhaltung:

$$
\vec{v}^{k}=\frac{\vec{v}^{i} R_{\mathrm{n}}^{i^{3}}+\vec{v}^{j} R_{\mathrm{n}}^{j^{3}}}{R_{\mathrm{n}}^{k^{3}}}
$$

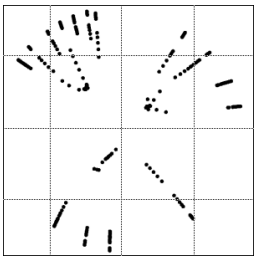


Die gerichtete Diffusion wird ebenfalls berücksichtigt und ist abhängig von der Entgasung der Flüssigkeit sowie der Art der auftretenden Gasmoleküle. In den Abbildungen 2.20 sind typische Anwachsraten von Kavitationsblasen im Parameterraum von Schalldruckamplitude und Ruheradius aufgetragen. Die Werte werden in der Simulation aus einer Tabelle übernommen.

Im Allgemeinen sind in Vielblasensystemen die freien Wegstrecken der Blasen so gering, dass die Zeitspannen zwischen Blasenkollisionen zu kurz sind, als dass es zu einer deutlichen diffusionsbedingten Änderung der Blasengröße kommen könnte. Die Implementation der Partikelmodellierung bietet die Option die Änderungsrate des Ruheradius nach der Wachstumsrate (2.35) einzubeziehen.

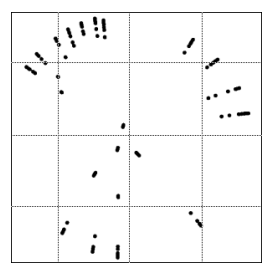




\section{Kapitel 5}

\section{Anwendungen der Partikelmodellierung}

In den aus Ultraschallanwendungen bekannten Vielblasensystemen lassen sich eine Vielzahl für die jeweiligen Bedingungen typische Strukturen erkennen. Von MetTin [82] wird ein Klassifikationsschema der bekannten Blasenstrukturen vorgestellt. Das folgende Kapitel zeigt einzelne Strukturen aus diesem „Zoo“ der Blasenformationen. Durch den Einsatz der in den vorherigen Kapiteln vorgestellten Modellierungen gelingt die numerisch Nachgebildung komplexer Figuren.

Die gesamte numerische Behandlung orientiert sich stets an beobachteten Blasenstrukturen, die teils in Hochgeschwindigkeits-Videoaufnahmen räumlich und zeitlich detailliert aufgelöst wurden und teils in Langzeitbelichtungen als einhüllende Struktur festgehalten wurden.

Der hier betrachtete Ausschnitt an Strukturen umfasst zunächst in Abschnitt 5.1 eine einzelne lasergenerierte Blase. An dieser Einzelblase kann die Wirkung der primären Bjerkneskraft in einem Stehwellenfeld sehr gut verifiziert werden. In Abschnitt 5.2 wird die Wechselwirkung mit einer zweiten Blase, wie sie von der sekundären Bjerkneskraft beschrieben wird, untersucht.

Nach dieser ersten quantitativen Überprüfung der Modellierungen werden in Abschnitt 5.3 die von APPEL [83] dreidimensional aufgenommenen Mehrblasensysteme („akustische Lichtenbergfiguren“) numerisch nachgebildet. Für die Betrachtung der in einem Vielblasensystem auftretenden Ruheradien wird in Abschnitt 5.4 ein erweiterter experimenteller Aufbau von APPEL [83] eingesetzt.

Eine weitere typische Struktur besteht aus einer doppelschichtigen paarweisen Anordnung von Streamerfiguren, wie sie bereits von MetTin et al. [84, 85] beschrieben wurde. In Abschnitt 5.5 wird die Ausbildung solcher Figuren auf die Schallfeldgeometrie zurückgeführt und qualitativ berechnet.

In Abschnitt 5.6 wird auf von KReFting [19] gemachte Aufnahmen zurückgegriffen, in denen es durch die gezielte Einbringung von bevorzugten Blasenentste-

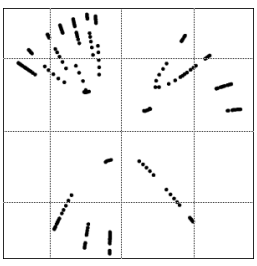


hungspunkten möglich ist, diese Positionen festzulegen und entsprechend genau in die Simulation einzusetzen.

Um die Rückwirkung einer Blasenverteilung auf das Schallfeld zu analysieren, wird in Abschnitt 5.7 die von KREFTING [19] vorgestellte zweidimensionale Lichtenbergfigur verwendet.

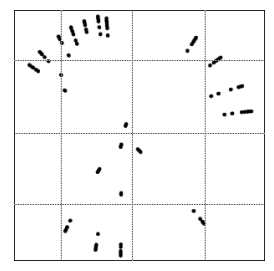




\subsection{Lasererzeugte Einzelblasen}

In dieser Versuchsreihe wird eine einzelne Blase außerhalb des Druckbauchs präpariert und ihre Trajektorie verfolgt. In der ruhenden Flüssigkeit wandert die Blase in Richtung Druckbauch des im Resonator ausgebildeten Stehwellenfeldes der $(1,1,1)$-Mode.

Die Versuchsanordnung ist in Abbildung 5.1 gezeigt. Die zu untersuchenden Blasen werden mit Hilfe eines in leicht entgastem Wasser fokussierten Femtosekunden-Lasers generiert. Der Aufbau geht auf die Arbeit von Geisler [86] zurück. Durch dieses kontaktlose Einbringen der Blasen bleiben Resonator und Schallfeld weitestgehend unbeeinflusst. Außerdem lassen sich durch das Lasersystem Blasen zu definierten Zeitpunkten und an Positionen mit $100 \mu \mathrm{m}$ Genauigkeit erzeugen. Mit Einstellung der Laserpulsenergie wird zudem die Blasengröße vorgegeben. Im optischen Durchbruch kommt es zunächst zu einer Plasmabildung, die eine entlang der Strahlachse ausgedehnte Blasenstruktur zur Folge hat. Wie von GeIsler [86] untersucht, bildet sich nach wenigen Pulsationsperioden eine einzelne sphärische Blase aus.

Da es nicht notwendig ist, den Zeitpunkt des optischen Durchbruchs exakt zu erfassen, kann das Femtosekunden-Lasersystem zur Blasenerzeugung unabhängig von dem Küvetten- und Kameraaufbau betrieben werden. Die freilaufende HiSISHochgeschwindigkeitskamera signalisiert jeden Anfang einer Belichtung (Auflösung: $54 \mu \mathrm{m} /$ Pixel in der Bildhöhe und $27 \mu \mathrm{m} /$ Pixel in der Bildbreite). Der so freigegebene Trigger löst den LED-Blitz nun mit dem nächsten Takt des Funktionsgenerators aus (Periodenlänge $\tau_{\text {osz }} \approx 50 \mu \mathrm{s}$ ), wodurch eine Synchronisation der Belichtung mit der Schallfeldphase erreicht wird. Über die Verzögerung kann die gewünschte Phasenlage des die Blasen treibenden Stehwellenfeldes abgepasst werden. Weitere Details des verwendeten Aufbaus sind in Anhang B angegeben.

Das Eigenmoden-Spektrum des hier eingesetzten Resonatorsystems bestehend aus der mit leicht entgastem destillierten Wasser befüllten Hellma-Küvette mit Piezoring wurde in Abschnitt 3.2 bereits diskutiert.

Der über die Laserpulsenergie eingeregelte Ruheradius wird an einer Serie von Blasen ohne Schallfeld bestimmt. Dazu liefert die hochaufösende Pulnix-CCDKamera mit Fernfeldmikroskop (Auflösung $0.41 \pm 0.01 \mu \mathrm{m} /$ Pixel) Bilder wie in Abbildung 5.2 gezeigt. Der mittlere Blasenradius wurde bestimmt zu:

$$
R_{\mathrm{n}}=4.8 \pm 0.7 \mu \mathrm{m}
$$

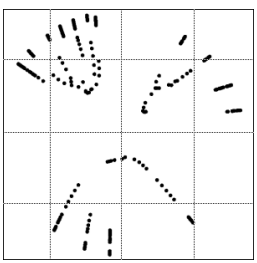




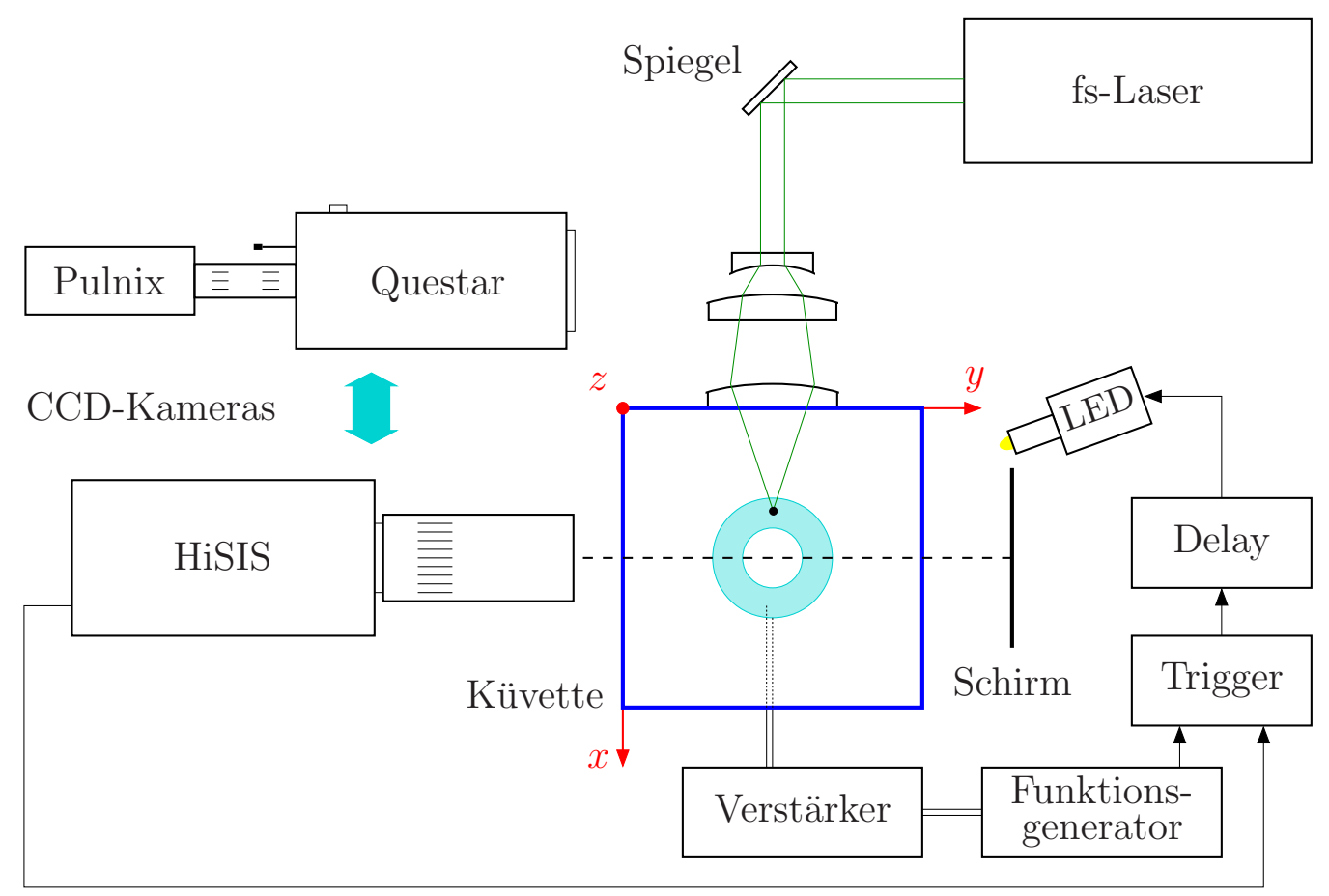

Abbildung 5.1: Schematische Darstellung (Aufsicht) des eingesetzten Aufbaus mit austauschbaren CCD-Kameras. Die Einkopplung des Schallfeldes erfolgt über den Piezoring unter dem Küvettenboden mittels des Funktionsgenerators, eines Verstärkers und einer Impedanzanpassung. Der Femtosekunden-Laserpuls wird über eine spezielle Aufweitungsoptik in die Küvette fokussiert. Der LED-Blitz wird über eine Triggerschaltung von der jeweiligen CCD-Kamera freigegeben und vom Funktionsgenerator ausgelöst. Aufnahmen können im Durchlicht (mit zusätzlicher Mattscheibe) oder im Streulicht gemacht werden.

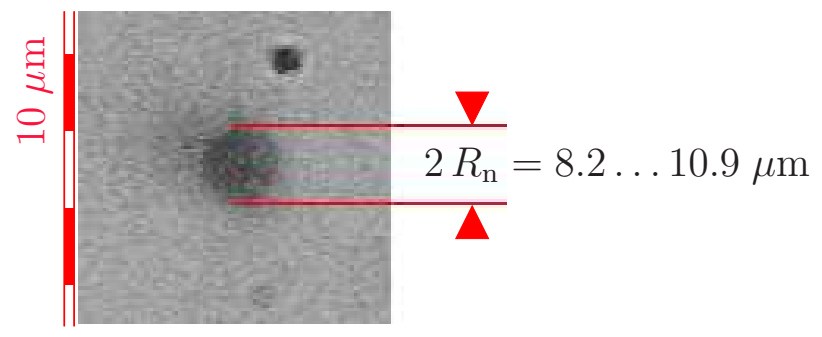

Abbildung 5.2: Ausgemessener Durchmesser einer nicht getriebenen Blase nach dem Laserschuss; Aufnahme der hochauflösenden Pulnix-CCDKamera im Durchlicht; der obere fixe Punkt ist auf eine Blase an der Küvettenwand zurückzuführen; Kameraauflösung $0.41 \pm 0.01 \mu \mathrm{m} /$ Pixel.

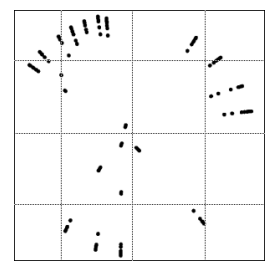




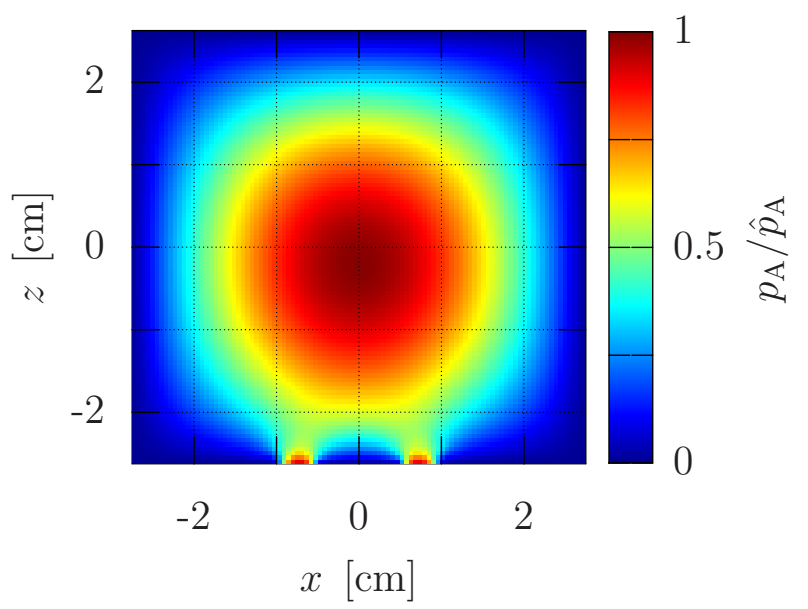

\begin{abstract}
Abbildung 5.3: Darstellung der normierten Schalldruckamplitude in der Hellma-Küvette als zeitharmonische Lösung der Helmholtz-Gleichung (3.3); der Druckbauch liegt etwas unterhalb der geometrischen Mitte bei $(0,0,-2) \mathrm{mm} ; \nu=23.2 \mathrm{kHz}$.
\end{abstract}

Die Schallfeldmode ergibt sich als zeitharmonische Lösung der in Abschnitt 3.2 diskutierten Helmholtz-Gleichung (3.3). Abbildung 5.3 zeigt die Schalldruckverteilung für die experimentell vorgegebene Frequenz:

$$
\nu=23.2 \mathrm{kHz}
$$

Die normierte Schalldruckverteilung im Resonator wird nach Referenzmessungen mit einem Glasfaserhydrophon (siehe Anhang B) über die am Piezokristall anliegende Spannung rückskaliert auf eine maximale Schalldruckamplitude von:

$$
\hat{p}_{\mathrm{A}}=120 \pm 10 \mathrm{kPa}
$$

Durch die Fokussierung des optischen Durchbruchs in der $x$-z-Ebene kann die aufgenommene und in Abbildung 5.4 aufgetragene Trajektorie der entstehenden Blase als zweidimensionale Bahn ausgewertet werden $(y=0)$. Das Bild wurde aus dem im Streulicht aufgenommenen Film generiert, indem aus jedem Einzelbild die Spalte ( $z$-Achse) bzw. Zeile ( $x$-Achse) mit dem hellsten Lichtpunkt extrahiert wurde und dann aufeinanderfolgende Spalten bzw. Zeilen nebeneinander gestellt wurden.

Nach der Induzierung durch den Laserpuls $(t<0)$ startet die Blase mit Geschwindigkeit Null. Durch die primäre Bjerkneskraft zum Druckbauch hin beschleunigt

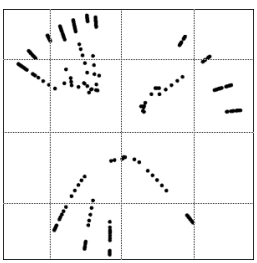


erreicht sie eine maximale Geschwindigkeit, die sich aus der Tangentensteigung um $t=0.2 \mathrm{~s}$ in Aufnahme 5.4 bestimmt:

$$
v_{\mathrm{x}}^{\max } \approx 27 \mathrm{~mm} / \mathrm{s}
$$

An der Intensität der in Abbildung 5.4 aufgetragenen Trajektorie kann ein diffusionsbedingtes Anwachsen der Blase erkannt werden.

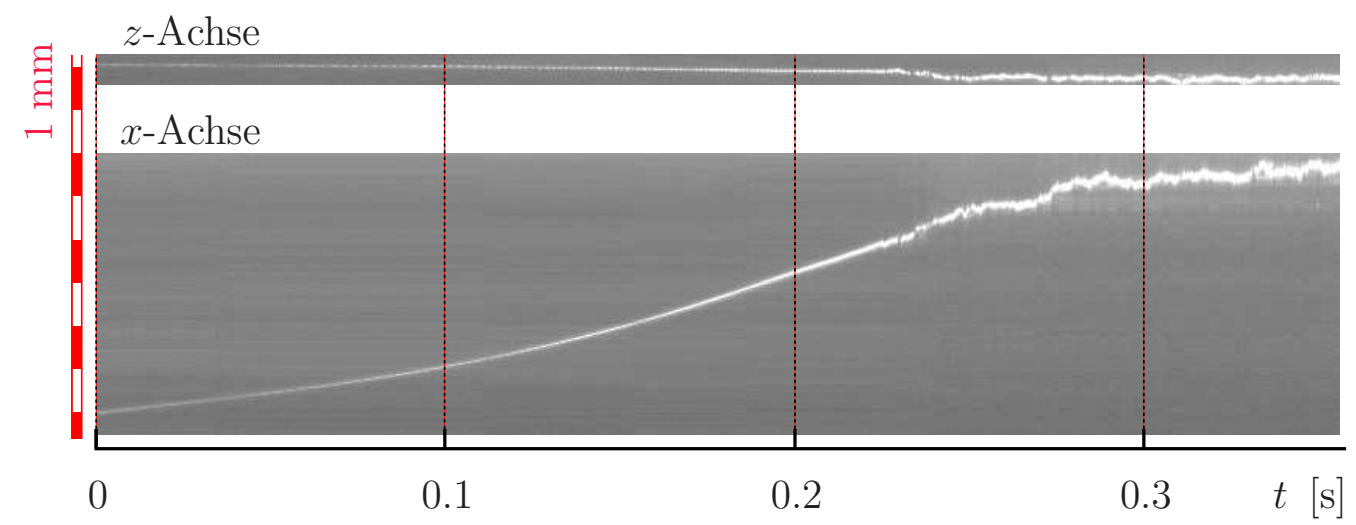

Abbildung 5.4: Darstellung der aufgenommenen Trajektorie einer einzelnen ins Zentrum des Resonators laufenden Blase (Aufgrund der Kameraauflösung skalieren $x$ - und $z$-Achse unterschiedlich); Bildrate: $2250 \mathrm{~Hz}$, Ortsauflösung $54 \mu \mathrm{m} /$ Pixel in der $z$-Achse und $27 \mu \mathrm{m} /$ Pixel in der $x$ Achse.

In der Nähe des Druckmaximums ab etwa $t \approx 0.225 \mathrm{~s}$ beginnt die durch Diffusion angewachsene Blase „zappelnde“ Bewegungen auszuführen, während sie tendenziell weiter in den Druckbauch gezogen wird. Aufgrund der langen Belichtungszeit gegenüber dieser schnellen Translation verschmiert die Aufnahme. Die Ursache dieses Verhaltens besteht darin, dass die Blase sich in Parameterbereichen ihrer Oberflächenstabilitätsgrenze befindet. Somit kommt es zu einer ständigen Abgabe von Mikroblasen und deren Rekombination. Die abgespaltenen Mikroblasen liegen im Allgemeinen unterhalb der Auflösungsgrenze. Abbildung 5.5 zeigt in einer hochaufgelösten Zeitserie den Abspaltungsprozess an einer ähnlichen oberflächeninstabilen Blase und die Rekombination der Teilblasen in einem anderen Experiment. Dieses Verhalten wurde bereits von Krefting [19] und KocH [87] untersucht.

Für die Erfassung der Blasentrajektorie in Abbildung 5.6 wurden von LuTHER [81] und APPEL [83] implementierte Filter- und Trackingalgorithmen eingesetzt.

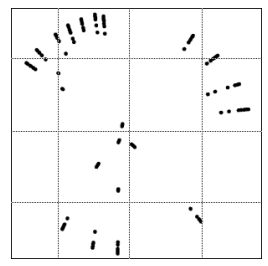




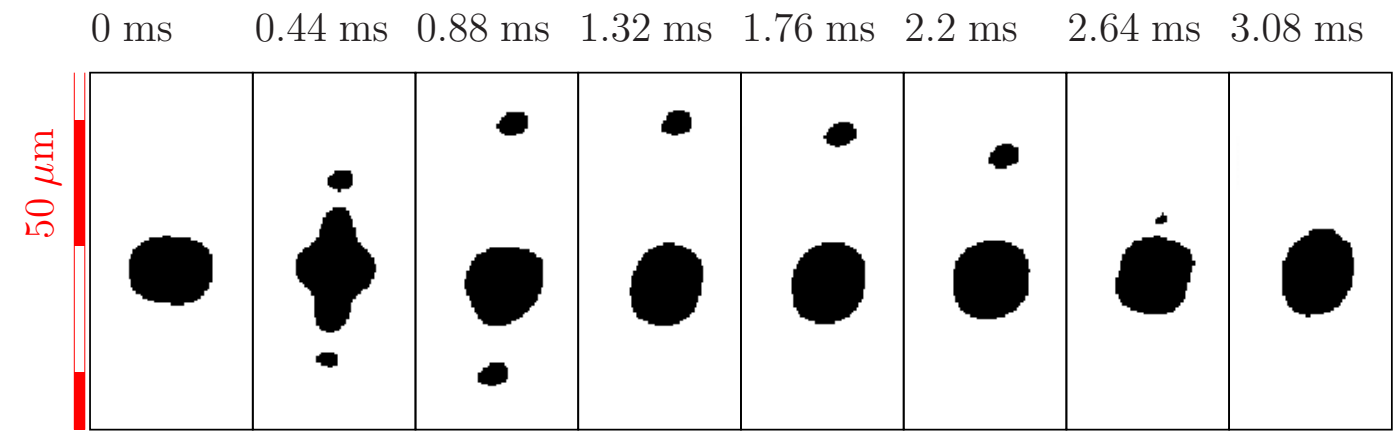

Abbildung 5.5: Abspaltung zweier Mikroblasen von einer oberflächeninstabilen Blase und deren Rekombination; bildbearbeitete Aufnahme von Krefting et al. [88, 19].

Der Nullpunkt wird durch das geometrische Zentrum des Resonators festgelegt ${ }^{1}$.

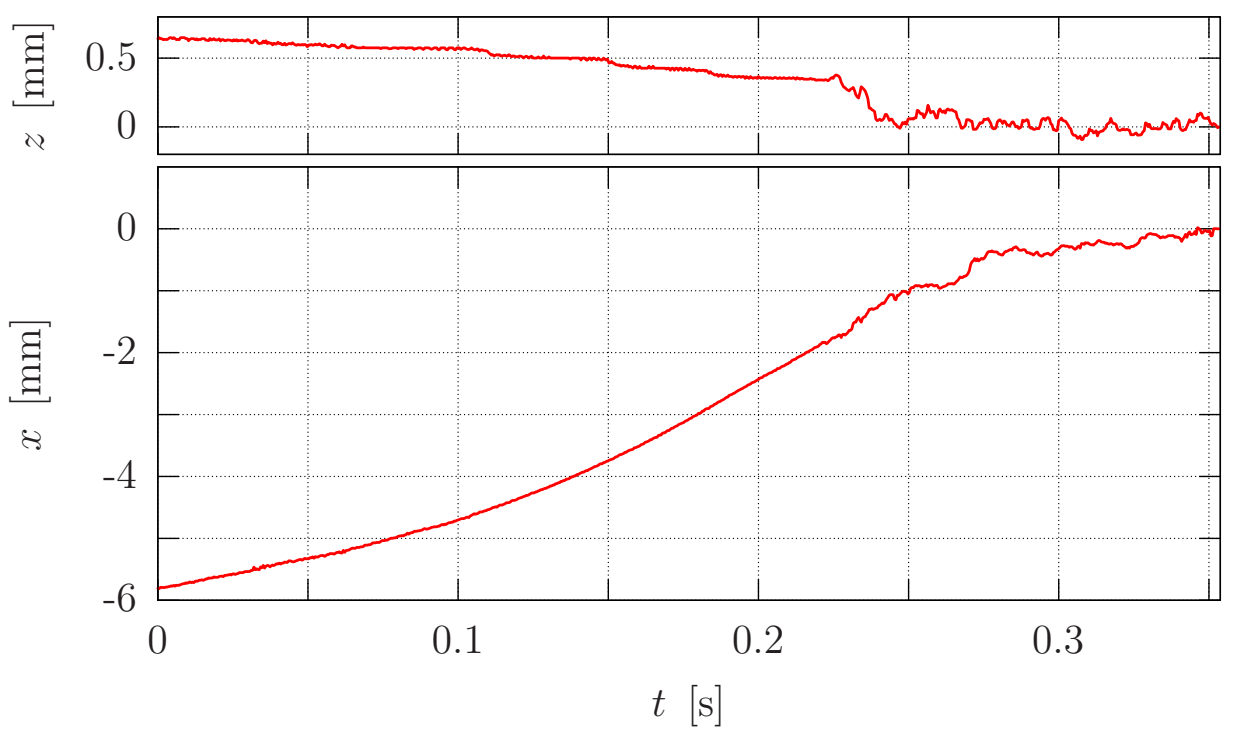

Abbildung 5.6: Nach Filterung und Tracking aus Aufnahme 5.4 gewonnene Trajektorie ( $x$ - und $z$-Achse skalieren unterschiedlich).

${ }^{1}$ Durch die in Abschnitt 3.3.5 betrachtete Resonanzverschiebung und die Asymmetrie durch den Schallwandler am Boden kommt es nicht zu einer exakten Übereinstimmung mit dem Druckbauch, das heißt der Ort maximaler Schalldruckamplitude liegt hier $2 \mathrm{~mm}$ tiefer.

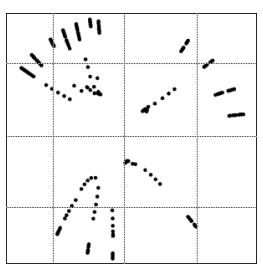




\subsubsection{Partikelmodellierung}

Zum Einsatz kommt hier die Partikelsimulation nach Abschnitt 4.5 unter Einbeziehung von primärer Bjerkneskraft, Reibung und virtueller Masse:

$$
\vec{F}_{\mathrm{Bp}}+\vec{F}_{\mathrm{D}}+\vec{F}_{\mathrm{M}}=\overrightarrow{0}
$$

Zur Initialisierung dienen experimentell vorgegebenen Parameter:

$$
\begin{aligned}
\text { maximale Schalldruckamplitude: } & \hat{p}_{\mathrm{A}}=120 \pm 10 \mathrm{kPa} \\
\text { Schallfeldfrequenz: } \quad \nu & =23.2 \mathrm{kHz}
\end{aligned}
$$

Die Startwerte der Blasentrajektorie ergeben sich durch die Auswertung der Aufnahmen: der Ruheradius nach Abbildung 5.2, die Startposition aus der an der Lage des Druckbauches ausgerichteten Trajektorie aus Abbildung 5.6, ebenso die Anfangsgeschwindigkeit aus der Tangentensteigung zu Zeitpunkt $t=0$ :

$$
\begin{aligned}
\text { Ruheradius: } & R_{0}=4.8 \pm 1 \mu \mathrm{m} \\
\text { Startposition: } & \vec{x}_{0}=(-6.3,0,-1.3) \pm 0.5 \mathrm{~mm} \\
\text { Anfangsgeschwindigkeit: } & \vec{v}_{0}=(6.5,0,0.5) \pm 0.5 \mathrm{~mm} / \mathrm{s}
\end{aligned}
$$

Die Simulation berücksichtigt entsprechend Abschnitt 2.5 ein Anwachsen der Blase durch gerichtete Diffusion gemäß der Anwachsrate (2.35):

$$
R_{\mathrm{n}}=R_{\mathrm{n}}(t), \quad R_{0}=R_{\mathrm{n}}(t=0)
$$

Unmittelbar nach der Plasmabildung im optischen Durchbruch formt sich eine Blase vermutlich überwiegend aus Wasserstoff- und Sauerstoff-Molekülen. Nach kurzer Zeit wird es zu einem Ausgleich mit Gasbestandteilen der im Wasser gelösten Luft (78\% Stickstoff, $21 \%$ Sauerstoff und $1 \%$ Edelgasen) kommen, das heißt einem wesentlichen Anteil von Stickstoff-Molekülen.

Die Werte für die Diffusionsrate $D_{\mathrm{R}}$ und Sättigungskonzentration $c_{\text {sat }}$ werden für eine Temperatur von $\Theta=298.15 \mathrm{~K}$ bei TöGEL und LoHSE [54] angeben:

\begin{tabular}{lcc} 
Spezies & $D_{\mathrm{R}}\left[10^{-9} \mathrm{~m}^{2} / \mathrm{s}\right]$ & $c_{\text {sat }}\left[\mathrm{mol} / \mathrm{m}^{3}\right]$ \\
\hline Wasserstoff $\mathrm{H}_{2}$ & 5.1 & 0.78 \\
Sauerstoff $\mathrm{O}_{2}$ & 2.4 & 1.3 \\
Stickstoff $\mathrm{N}_{2}$ & 2 & 0.6 \\
Argon Ar & 2.5 & 1.4 \\
Luft & 2.09 & 0.76
\end{tabular}

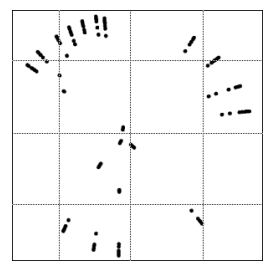




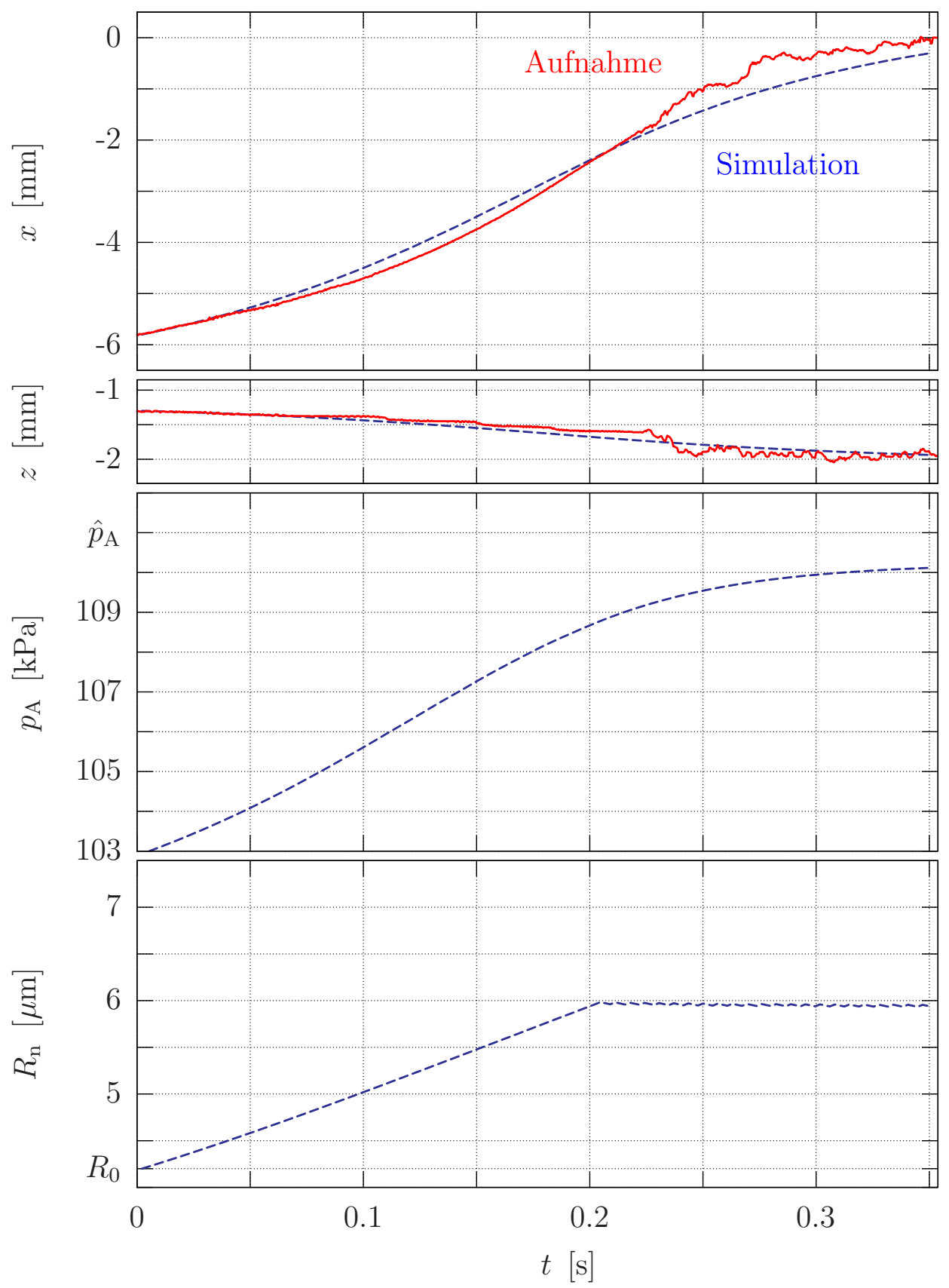

Abbildung 5.7: Partikelsimulation einer einzelnen in ihre Ruhelage gezogenen Luft-Blase gegenüber der experimentellen Aufnahme. Gezeigt sind die $x$ - und $z$-Komponente der Trajektorie, die Schalldruckamplitude am Ort der Blase $p_{\mathrm{A}}$ sowie der Ruheradius der Blase $R_{\mathrm{n}} ; \hat{p}_{\mathrm{A}}=111 \mathrm{kPa}$, $\nu=23.2 \mathrm{kHz}, \eta=1$ (isotherm), $R_{0}=4.2 \mu \mathrm{m}, D_{\mathrm{R}}=2.09 \cdot 10^{-9} \mathrm{~m}^{2} / \mathrm{s}$ (Luft), $c_{\mathrm{sat}}=0.76 \mathrm{~mol} / \mathrm{m}^{3}, c_{\infty} / c_{\mathrm{sat}}=100 \%, \alpha=11$.

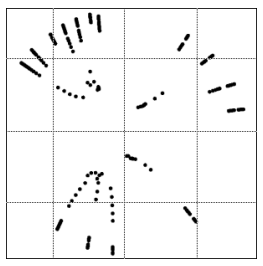


In Abbildung 5.7 (unten) ist der anwachsende Ruheradius aufgetragen. Es ist zu erkennen, dass die Blase von $R_{0}=4.2 \mu \mathrm{m}$ bis knapp unter $R_{\mathrm{n}}=6 \mu \mathrm{m}$ anwächst, wo sie in den oberflächeninstabilen Bereich eintritt. Diese Stabilitätsgrenze ist auch in der Parameterraumebene von Ruheradius und Schalldruckamplitude in Abbildung $5.8 \mathrm{zu}$ erkennen. Zusätzlich zu der aus Abbildung 2.15 bekannten parametrischen Stabilitätsgrenze sowie der analog zu den Abbildungen 2.20 aufgetragenen Gleichgewichtslage der gerichteten Diffusion ist hier die Grenze der optischen Auflösung eingetragen. Die Aufösungsgrenze ergibt sich aus den Parameterpaaren von Schalldruckamplitude und Ruheradius, zu denen die radial pulsierende Blase über ihre Schwingungsperiode betrachtet einen maximalen Radius von $10 \mu \mathrm{m}$ erreicht, erst Blasen dieser Größe werden mit der eingesetzten Optik detektiert ${ }^{2}$.

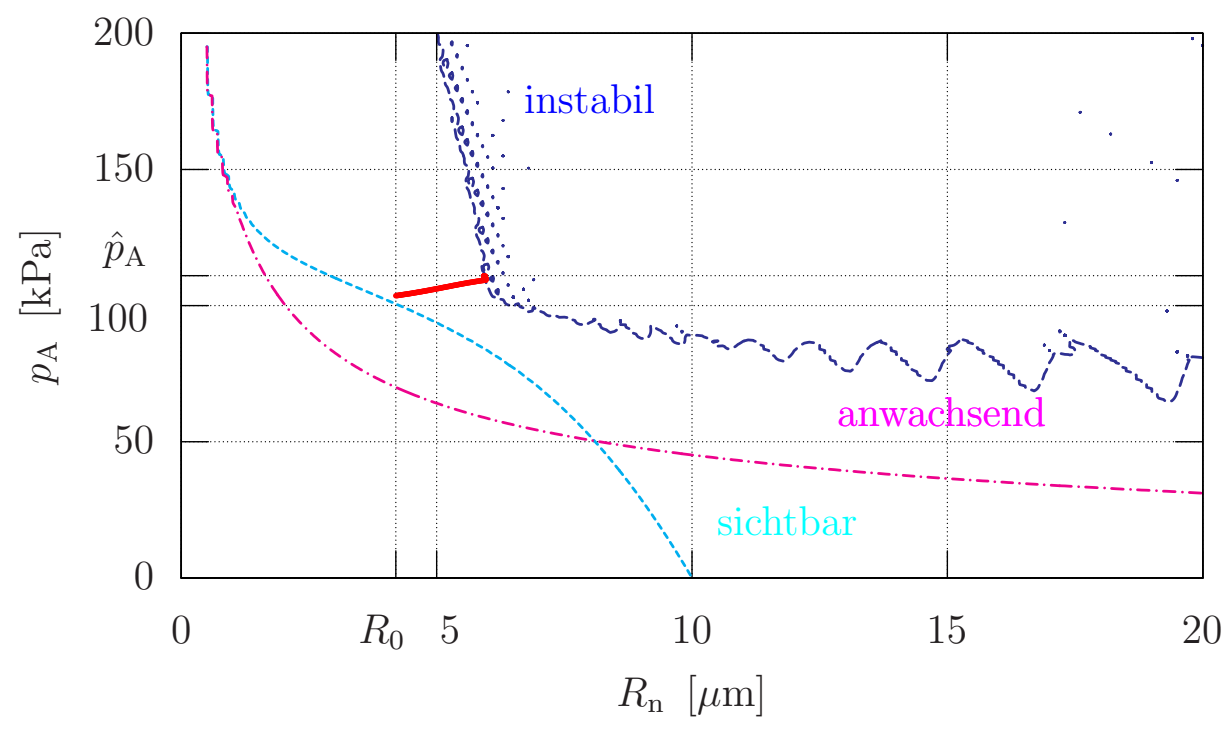

Abbildung 5.8: Betrachtung der Blasenbewegung (rote Linie) in der Schalldruck-Ruheradius-Parameterebene. Oberhalb der Diffusionsschwelle wachsen die Blasen an bis zum Erreichen der Stabilitätsgrenze. Optisch aufgelöst werden können Blasen, deren maximaler Radius die $10 \mu \mathrm{m}$ Grenze erreicht; $R_{0}=R_{\mathrm{n}}(t=0)=4.2 \mu \mathrm{m}, \nu=23.2 \mathrm{kHz}, \hat{p}_{\mathrm{A}}=111 \mathrm{kPa}$.

In der Simulation werden Blasen oberhalb ihrer parametrischen Stabilitätsgrenze um das Volumen einer angenommenen abgespaltenen Mikroblase mit $R_{\mathrm{n}}=1.5 \mu \mathrm{m}$ reduziert. Nach Abbildung 5.8 liegen diese Blasen unterhalb der Auflösungsgrenze. Die Simulation setzt an dieser Stelle jedoch keine neuen Bla-

2 Im Streulicht aufgenommene Blasen streuen einen Lichtkegel in das Kameraobjektiv, wodurch auch Blasengrößen unterhalb der optischen Aufösungsgrenze erfaßt werden können.

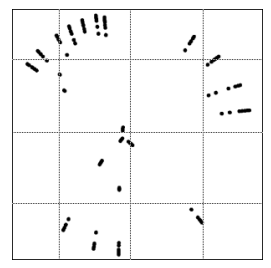


sen ein, so dass über den gesamten Zeitraum von einem Einblasensystem ausgegangen wird. Das im Experiment ab etwa $t \approx 0.25$ s einsetzende „Zappeln“ der Blasentrajektorie geht auf die bereits erwähnte Rekombination mit zuvor abgespaltenen Mikroblasen zurück und wird entsprechend von der Simulation nicht nachgebildet.

Die Reibung wird nach der in Abschnitt 4.4 ausgeführten Betrachtung entsprechend den Ergebnissen von KREFTing [19] eingesetzt in der Form (4.12):

$$
\overrightarrow{F_{\mathrm{D}}}=-11 \pi \mu R \vec{v}
$$

Die dynamische Anpassung des Polytropenexponenten nimmt wie in Abbildung 2.11 gezeigt kaum Einfluss auf das Schwingungsverhalten einer Blase. Zur Vereinfachung der Simulation wird daher hier der isotherme Fall behandelt $(\eta=1)$.

Die physikalisch sinnvollen Parameterbereiche liefern Kurvenscharen die in Abbildung 5.9 gezeigt sind, sie verdeutlichen den unterschiedlich starken Einfluss der jeweiligen Parameter auf die Blasentrajektorie. Am stärksten wirken sich die Wahl des Ruheradius $R_{0}=R_{\mathrm{n}}(t=0)$ sowie der maximalen Schalldruckamplitude $\hat{p}_{\mathrm{A}}$ aus. Die Wahl des Gases spielt dagegen bereits eine untergeordnete Rolle. Mit der in Abschnitt 4.4 diskutierten Unterscheidung in das Reibungsmodell der harten Kugel $(\alpha=6)$ und der Gasblase $(\alpha=12)$ wird die Simulation ebenfalls beeinflußt. Ruheradius und Schalldruckamplitude sind durch experimentelle Messungen relativ gut festgelegt, so dass in der Modellierung der Diffusion der größte Toleranzbereich liegt. Durch die Anpassung dieser Parameter an die experimentell bestimmte ebenfalls in Abbildung 5.9 eingezeichneten Trajektorie lassen sich die in Abbildung 5.7 eingesetzten Parameter festlegen: $R_{0}=4.2 \mu \mathrm{m}, \hat{p}_{\mathrm{A}}=111 \mathrm{kPa}$, Luft, $\alpha=11$. Nach dieser Betrachtung scheint die Modellierung aus primärer Bjerkneskraft, Reibung und virtueller Masse hinreichend, um das beobachtete Blasenverhalten zu erklären.

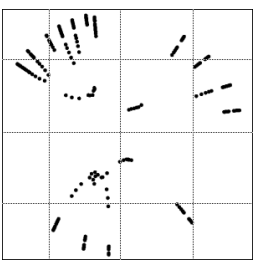




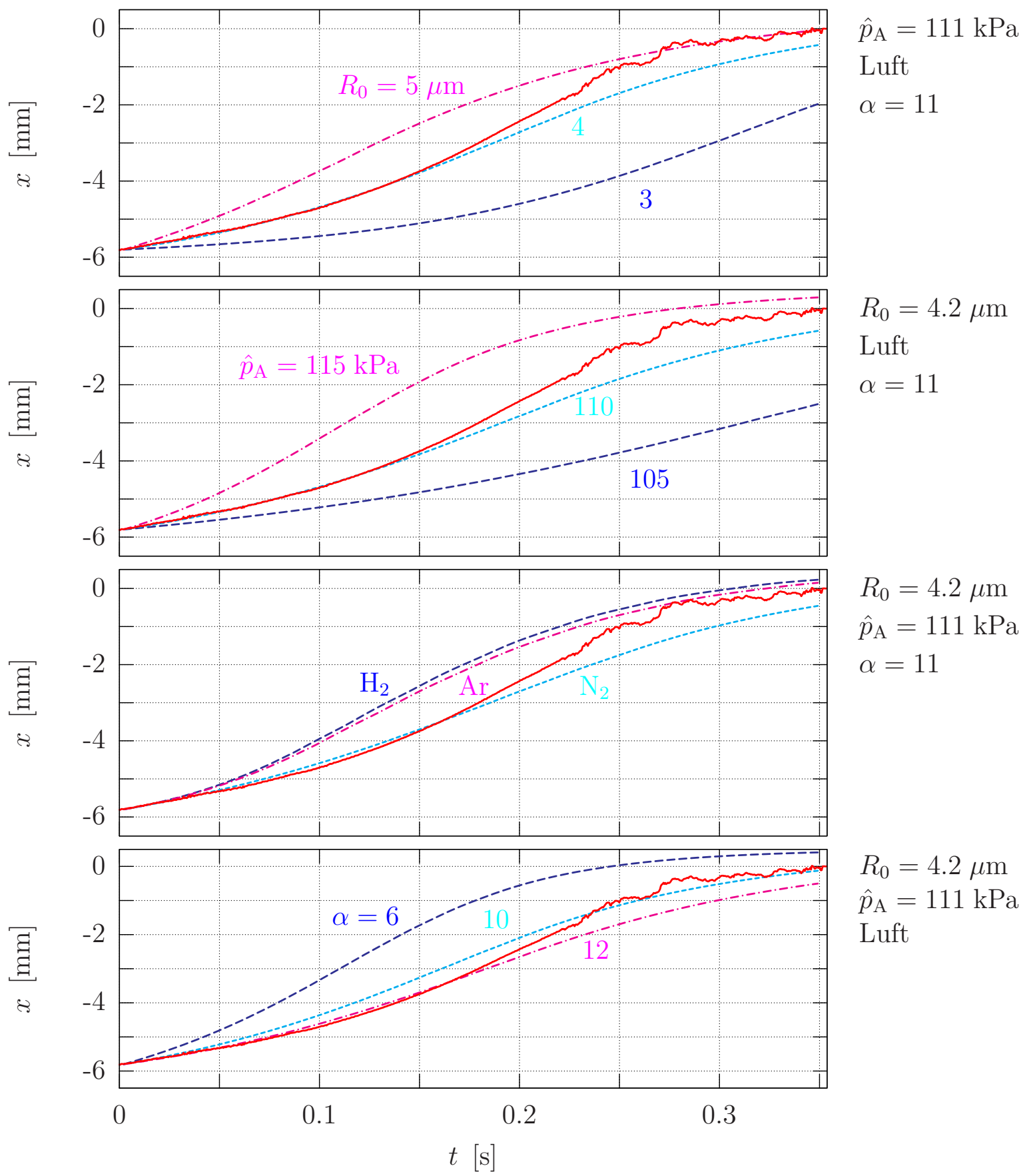

Abbildung 5.9: Darstellung der Lösung der Bewegungsgleichung für verschiedene Parametersätze vom Ausgangsruheradius, der Schalldruckamplitude, des diffundierenden Gases und dem Reibungskoeffizienten im Vergleich zu der in Abbildung 5.7 (oben) gezeigten experimentell bestimmten Blasentrajektorie (hier rot eingezeichnet); $R_{0}=3,4,5 \mu \mathrm{m}$, $\hat{p}_{\mathrm{A}}=105,110,115 \mathrm{kPa}$, Diffusion von $\mathrm{H}_{2}, \mathrm{~N}_{2}, \mathrm{Ar}, c_{\infty} / c_{\mathrm{sat}}=100 \%$, $\alpha=6,10,12$.

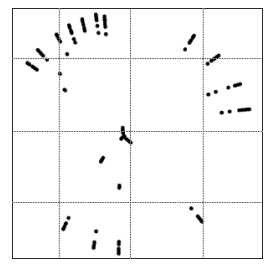




\subsection{Zweiblasen-Wechselwirkung}

Der experimentelle Aufbau entspricht dem in Abschnitt 5.1 für die Untersuchung von Einzelblasensystemen vorgestellten und der in Abbildung 5.1 gezeigten Anordnung.

Der Punkt des optischen Durchbruchs ist in dieser Versuchsreihe oberhalb des Druckbauchs auf die $z$-Achse eingestellt. Durch die Erzeugung zweier Blasen durch zwei aufeinander folgende Laserpulse kann zusätzlich zur Wirkung der primären Bjerkneskraft und der Reibung auch die Wechselwirkung unter dem Einfluss der sekundären Bjerkneskraft untersucht werden.

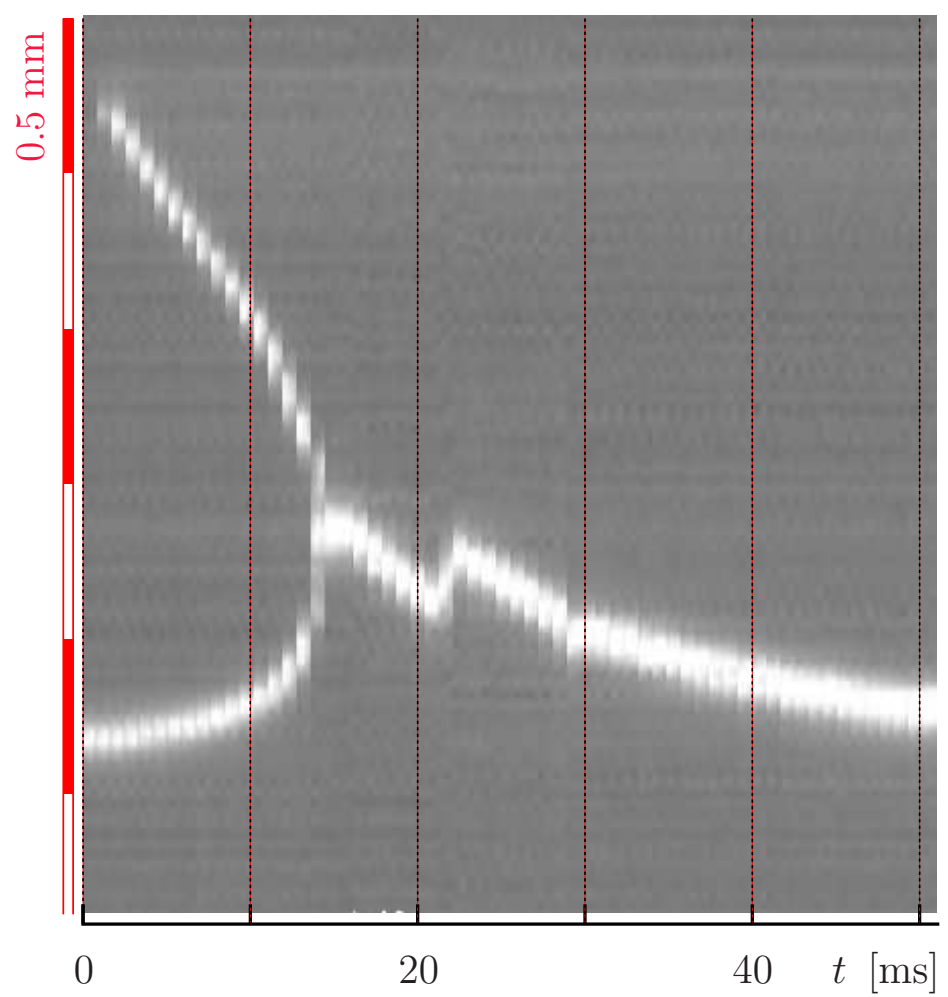

Abbildung 5.10: Darstellung der Kollision einer im Druckbauch befindlichen Blase und einer oberhalb mit optischem Durchbruch neu initialisierten Blase in entgastem destillierten Wasser. Erzeugung des "streak“Bildes wie bei Abbildung 5.4; Bildaufnahmerate 1125 Bilder/s, Auflösung $27 \mu \mathrm{m} /$ Pixel.

Die in Abbildung 5.10 dargestellte Aufnahme einer Zweiblasen-Wechselwirkung erfolgte im Streulicht mit einer Bildaufnahmerate von 1125 Bildern pro Sekunde bei einer optischen Auflösung von $27 \mu \mathrm{m} /$ Pixel (vertikal). Die Beobachtung

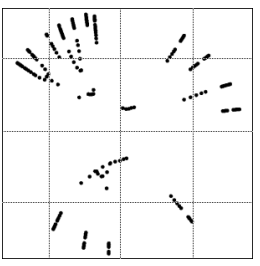


startet bei einer im Druckbauch des Stehwellenfeldes ruhenden Blase, die durch den ersten optischen Durchbruch induziert wurde. Die obere Blase wird mit dem Beginn der Aufnahme erzeugt und wandert zunächst entlang des Schalldruckgradienten in Richtung des Druckbauchs. Mit Annäherung der beiden Blasen aneinander setzt eine Wechselwirkung ein, wobei die untere Blase aus ihrer Ruhelage ausgelenkt wird und, wie auch die obere Blase, eine Beschleunigung in Richtung des Wechselwirkungspartners erfährt.

Nach der Kollision wandert die vereinigte Blase in Richtung ihrer Ruhelage im Druckbauch. Einzelne sprunghafte Bewegungen sind nach der Kollision zu erkennen. Diese sind typisch für Blasen, die aufgrund einer Kollision oder bei hohen Schalldrücken eine verstärkte Oberflächendynamik zeigen, in deren Folge es zu Abspaltungen von Teilblasen kommt. Deren Rekombination erscheint in dieser zeitlichen Auflösung „sprunghaft“. Oft liegen diese Teilblasen unterhalb der optischen Auflösungsgrenze. Dies Verhalten wurde bereits in Abschnitt 5.1 erläutert.

\subsubsection{Partikelmodellierung}

Die numerische Simulation des Zweiblasensystems liefert die in Abbildung 5.11 gezeigten Ergebnisse. Die dort eingesetzte Partikelsimulation geht von einer analytisch bestimmten $(1,1,1)$-Mode in dem Resonator aus. Die experimentell bestimmte maximale Schalldruckdruckamplitude liegt bei $\hat{p}_{\mathrm{A}}=120 \pm 10 \mathrm{kPa}$. In dieser Modellierung ist die isotherme Blasendynamik implementiert, siehe auch KoCH et al. [56].

Die Diffusion spielt hier nur eine untergeordnete Rolle, da die Zeitskala kürzer ist als im Experiment von Abbildung 5.4. Die Diffusionsprozesse laufen deutlich langsamer als die Zeitspanne bis zur Blasenkollision. Das heißt ein Anwachsen der Blasen erfolgt vor allem durch ihre Vereinigung.

Die gewählten Initialisierungswerte der Blasen sind:

\begin{tabular}{ccccc} 
Blase & $t_{\text {init }}[\mathrm{ms}]$ & $R_{\text {init }}[\mu \mathrm{m}]$ & $\vec{x}_{\text {init }}[\mathrm{mm}]$ & $\vec{v}_{\text {init }}[\mathrm{mm} / \mathrm{s}]$ \\
\hline 1 & 0 & 6 & $(0,0,0)$ & $(0,0,0)$ \\
2 & 1.78 & 5 & $(-0.21,0,2.25)$ & $(0,0,-71)$
\end{tabular}

Für diese Werte und den gewählten Druck $124 \mathrm{kPa}$ ergibt sich eine relativ gute Übereinstimmung von Theorie und Experiment. Die sprunghaften Abspaltungsund Vereinigungsprozesse werden von der Simulation nicht erfasst. 


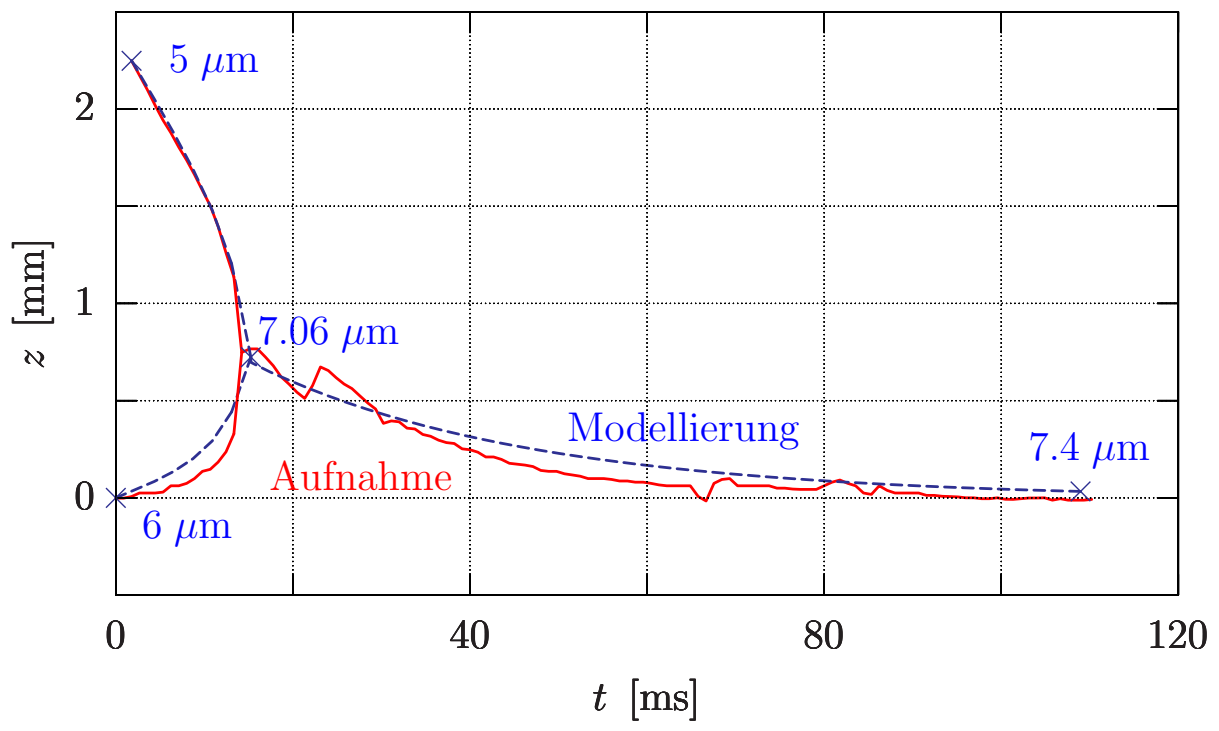

Abbildung 5.11: Numerische Simulation eines Zweiblasensystems durch die Partikelmodellierung. Unter der Annahme eines analytischen Stehwellenfeldes mit Druckbauch bei $z=0$. Die $\mu \mathrm{m}$-Angaben geben die Ruheradien zu den mit Kreuzen gekennzeichneten Zeitpunkten an; $\nu=22.945 \mathrm{kHz}$, $\hat{p}_{\mathrm{A}}=124 \mathrm{kPa}, \alpha=11, \eta=1$ (isotherm), $D_{\mathrm{R}}=2.09 \cdot 10^{-9} \mathrm{~m}^{2} / \mathrm{s}$, $c_{\mathrm{sat}}=0.76 \mathrm{~mol} / \mathrm{m}^{3}, c_{\infty} / c_{\mathrm{sat}}=30 \%$.

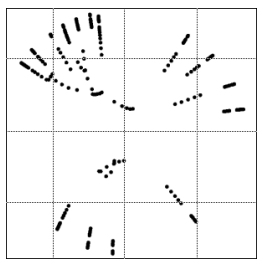




\subsubsection{Abspaltung}

Bei unveränderter Versuchsanordnung lassen sich auch Fälle wie in Abbildung 5.12 beobachten, in denen die zum Druckbauch gezogene Blase auf ihrem Weg eine Mikroblase abspaltet, die zunächst unterhalb der Auflösungsgrenze verweilt, jedoch durch gerichtete Diffusion anwachsen kann und sichtbar wird, um dann ihrerseits zum Druckbauch beschleunigt zu werden.

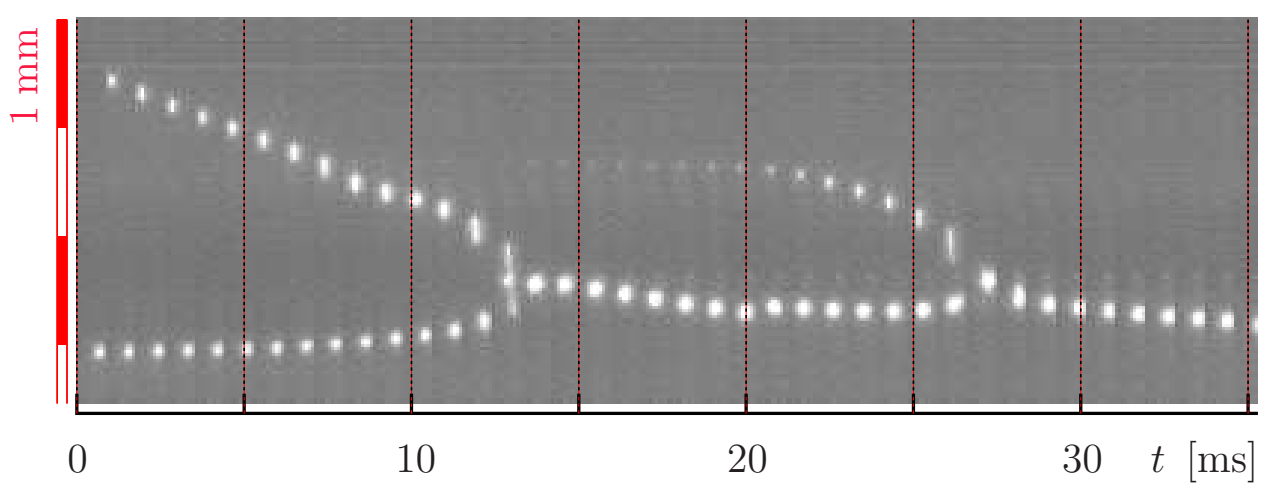

Abbildung 5.12: Aufnahme einer Blasenkollision mit vorheriger Abspaltung einer Mikroblase, die anwächst und ebenfalls zum Druckbauch gezogen wird, wo es zu einer zweiten Kollision kommt; entgastes destilliertes Wasser; $\nu=22.965 \mathrm{kHz}, \hat{p}_{\mathrm{A}}=120 \pm 10 \mathrm{kPa}$, Aufnahmerate 1125 Bilder pro Sekunde, Auflösung $27 \mu \mathrm{m} /$ Pixel.

Das Ergebnis einer Simulation mit angepassten Parametern (siehe auch KocH et al. [56]) ist in Abbildung 5.13 dem experimentell beobachteten Verlauf gegenübergestellt.

Die Initialisierung der Blasen erfolgte mit:

\begin{tabular}{ccccc} 
Blase & $t_{\text {init }}[\mathrm{ms}]$ & $R_{\text {init }}[\mu \mathrm{m}]$ & $\vec{x}_{\text {init }}[\mathrm{mm}]$ & $\vec{v}_{\text {init }}[\mathrm{mm} / \mathrm{s}]$ \\
\hline 1 & 0 & 7 & $(0,0,0)$ & $(0,0,0)$ \\
2 & 0.89 & 5.6 & $(-0.21,0,3.33)$ & $(0,0,0)$ \\
3 & 18.7 & 2.3 & $(-0.15,0,2.26)$ & $(0,0,0)$
\end{tabular}

Die beiden Ausgangsblasen zeigen in der Simulation bis zu ihrem Kollisionspunkt bei $t=13.3 \mathrm{~ms}$ ein schwaches diffusionsbedingtes Anwachsen, ihre Ruheradien zum Zeitpunkt der Kollision betragen $7.05 \mu \mathrm{m}$ bzw. $5.66 \mu \mathrm{m}$.

Die Verzögerung der $5.6 \mu \mathrm{m}$-Blase ist an dem Knick in ihrer Trajektorie bei $t \approx 8 \mathrm{~ms}$ und $z \approx 2.1 \mathrm{~mm}$ gut $\mathrm{zu}$ erkennen. Es ist anzunehmen, dass in dieser

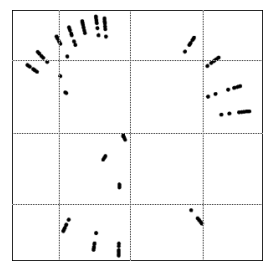


Phase die Mikroblase abgespalten wird, die später etwa an dieser $z$-Koordinate sichtbar wird. In der Simulation wird sie als $2.3 \mu \mathrm{m}$-Blase bei $t=18.7 \mathrm{~ms}$ und $z=2.26 \mathrm{~mm}$ nachträglich eingebracht. Das entspricht dem experimentell beobachteten Zeitpunkt und Ort. Bei $t=26.6 \mathrm{~ms}$ kollidiert sie mit der Hauptblase. In diesem Fall wurde der Druck auf $130 \mathrm{kPa}$ angepasst, wobei sich mit den gewählten Blasenradien ebenfalls eine relativ gute Reproduktion der experimentellen Blasenspuren ergibt.

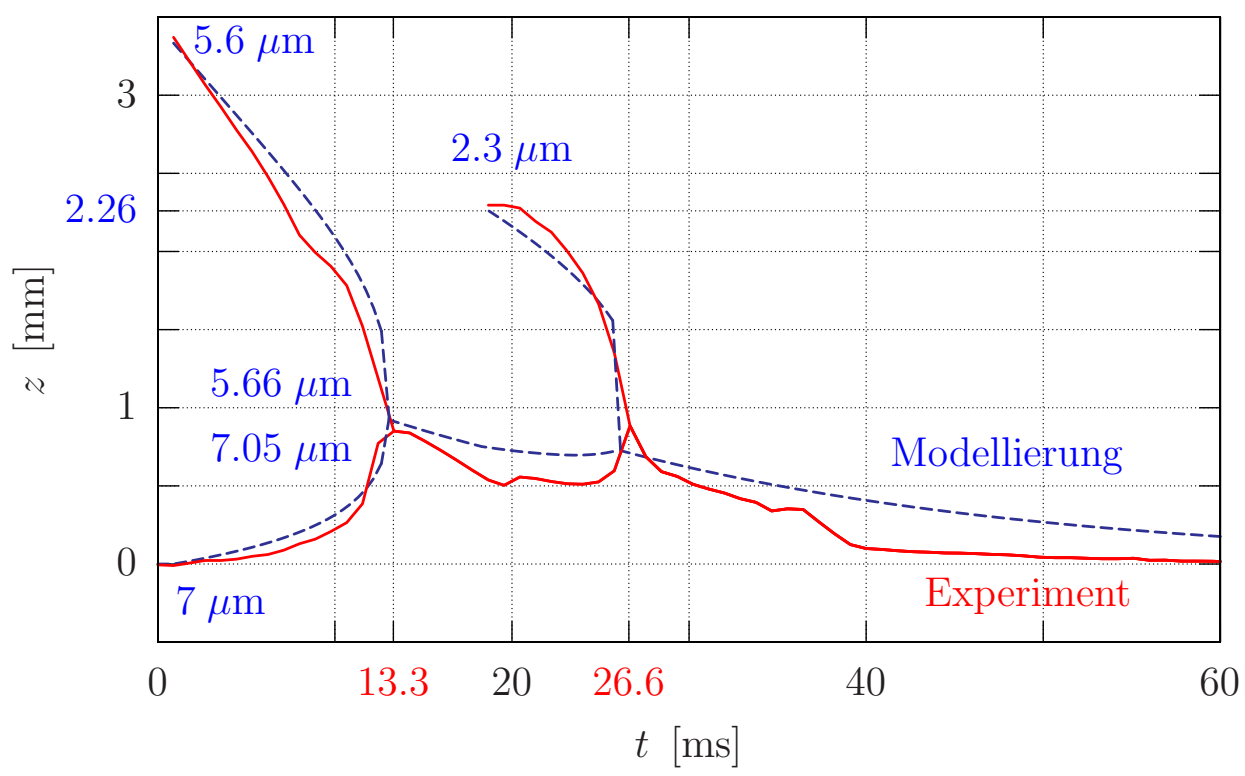

Abbildung 5.13: Gegenüberstellung der Blasentrajektorien der in Abbildung 5.12 gezeigten Aufnahme und einer Partikelmodellierung. Die $\mu \mathrm{m}$-Angaben an den Trajektorien zeigen die Ruheradien an; $\alpha=11$, $\hat{p}_{\mathrm{A}}=130 \mathrm{kPa}, \nu=22.965 \mathrm{kHz}, D_{\mathrm{R}}=2.09 \cdot 10^{-9} \mathrm{~m}^{2} / \mathrm{s}$ (Luft), $c_{\mathrm{sat}}=0.76 \mathrm{~mol} / \mathrm{m}^{3}, c_{\infty} / c_{\mathrm{sat}}=30 \%$.

Interessant ist hier die Beobachtung einer Mikroblase, die zunächst keinen Einfluss auf die Strukturbildung nimmt $\left(R_{\mathrm{n}} \lesssim 2 \mu \mathrm{m}\right)$. Die Blase zeigt ein auf gerichtete Diffusion zurückzuführendes Anwachsen, bis es zur beschriebenen Wechselwirkung mit der Hauptblase kommt $(t \approx 15 \mathrm{~ms})$.

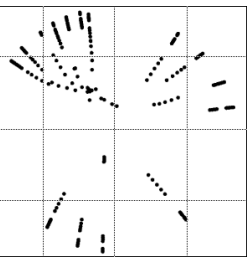




\subsection{Dreidimensionale Filamentfigur}

Abhängig von dem Schallfeld können sich in Vielblasensystemen unterschiedliche Figuren ausbilden. Eine typische Anordnung bei schwachen Stehwellenfeldern, die sich um einen Druckbauch aufbauen kann, ist die sogenannte akustische Lichtenbergfigur. Vom Erscheinungsbild her ist diese ähnlich der von Georg Christoph Lichtenberg beschriebenen möglichen Strukturbildung in einer elektrischen Entladung, die feine filamentartige Verästelungen ausformt.

Der Aufbau zur Aufnahme von Stereo-Hochgeschwindigkeitsfilmen dieser Blasenstrukturen ist in Abbildung 5.14 skizziert. Um die Blasentrajektorien in allen drei Raumdimensionen rekonstruieren zu können, wird ein stereoskopischer Kameraaufbau aus zwei baugleichen synchronisierten Hochgeschwindigkeitskameras verwendet. Technische Spezifikationen sind in Anhang B zusammengestellt. Die von APPEL [83] verwendete Küvette wird mit Leitungswasser befüllt. Zur Kavitation kommt es in diesem Aufbau mit dem Überschreiten der Kavitationsschwelle bei steigendem Schalldruck.

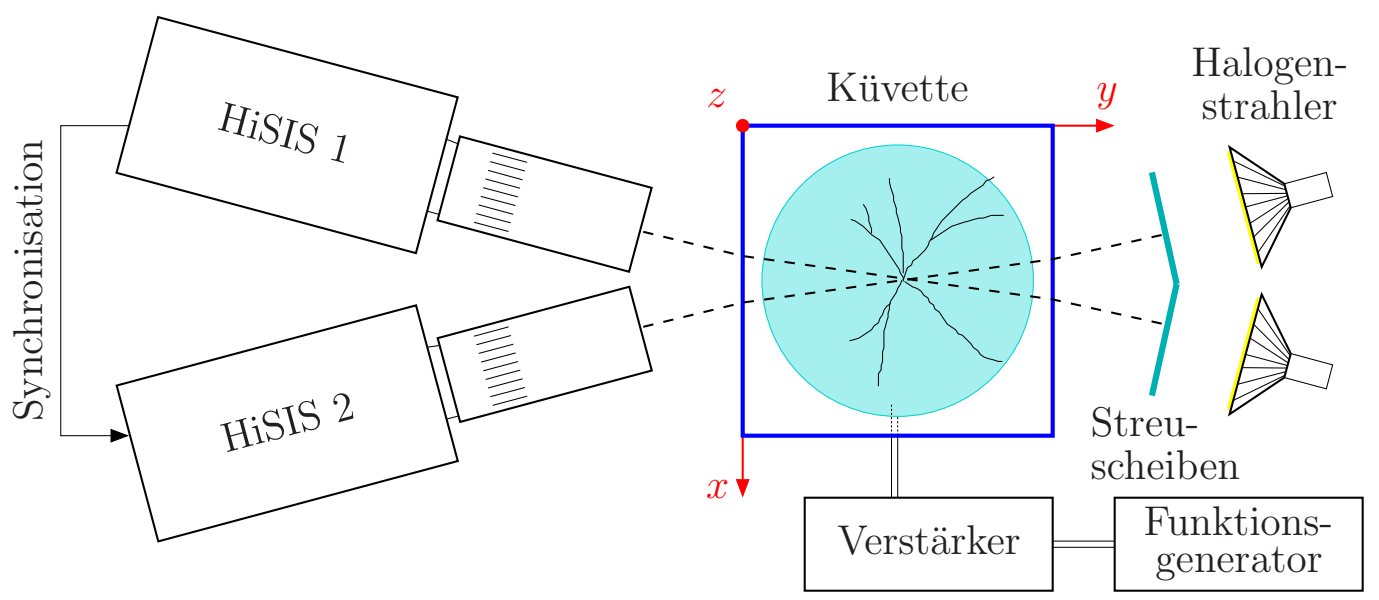

Abbildung 5.14: Schematische Skizze des Aufbaus zur stereoskopischen Hochgeschwindigkeitsaufnahme von Blasenstrukturen.

Die erste HiSIS-Kamera wird gestartet und nimmt frei laufend eine Filmsequenz von $7.28 \mathrm{~s}$ auf. Über die Triggerung wird die Belichtung der zweiten HiSIS-Kamera synchron ausgelöst. Das Signal des Funktionsgenerators ist abgestimmt auf die Resonanzlage des Küvettensystems. Es wird über den an der Resonatorunterseite verklebten Ultraschallwandler eingekoppelt und regt eine (1, 1, 1)-Mode an. Überschreitet die eingekoppelte Schallleistung die Kavitationsschwelle, kommt es zur akustischen Blasenbildung und entsprechend der Schallfeldstruktur zur Ausbildung von filamentartigen Blasenfiguren, den sogenannten Streamerarmen.

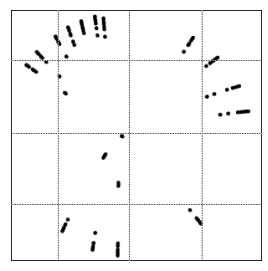


Die Auswertung der stereoskopischen Hochgeschwindigkeitsaufnahmen durch entsprechende Filter sowie 3d-Rekonstruktion und 3d-Verfolgung („Tracking“) liefert dreidimensionale Streamerstrukturen. Deren Nachbildung wurde nun in der Simulation quantitativ versucht.

Die experimentellen Systemparameter sind:

Schallfeldfrequenz: $\quad \nu=23.621 \mathrm{kHz}$

Schalldruckamplitude: $\hat{p}_{\mathrm{A}} \approx 160 \ldots 210 \mathrm{kPa}$

Die stereoskopischen Aufnahmen wurden von APPEL et al. [89] mit einer Bildrate von 2250 Bilder/s gemacht, wobei die HiSIS Kameras eine Auflösung von $256 \times 128$ Pixel liefern. Verwendet sind Objektive mit Nahlinsen. Die Kalibrierung ergibt eine Auflösung von $40 \mu \mathrm{m} /$ Pixel (horizontal) und $80 \mu \mathrm{m} /$ Pixel (vertikal). Abbildung 5.15 zeigt den Ausschnitt einer stereoskopischen Hochgeschwindigkeitsfilmsequenz.

Zur Rekonstruktion der Blasenpositionen aus den Aufnahmen wird ein von Luther [81] vorgestellter Algorithmus eingesetzt. Dabei werden die Aufnahmen im Wesentlichen kontrastverstärkt, um mit einem Schwellwertfilter die Einzelblasen herausheben zu können. Von Appel et al. [89, 90, 83] werden mit diesem Verfahren die Blasenpositionen in der stereoskopischen Bildsequenz getrennt für die rechte und linke Kamera detektiert. Die anschließende Zuordnung der Blasen aus beiden Kamerabildern zur Positionsbestimmung im dreidimensionalen Raum wird mittels Simulated Annealing ${ }^{3}$ optimiert.

Abbildung 5.16 zeigt die dreidimensionale Rekonstruktion der Streamerfigur. Der Nullpunkt ist hier zunächst beliebig gesetzt, er stimmt nicht zwangsweise mit dem geometrischen Zentrum der Küvette überein. Bei dem hier eingesetzten Resonator ist die Abmessung des piezokeramischen Wandlers gegenüber der Küvettenausdehnung nicht zu vernachlässigen, wodurch sich im Vergleich zu der früher etwa in Abbildung 3.4 (links) betrachteten Hellma-Küvette eine deutliche Verschiebung des Druckbauches der $(1,1,1)$-Mode aus dem Küvettenzentrum ergibt. Erst mit Kenntnis der Schallfeldgeometrie lässt sich die Figur in Beziehung zum Schalldruckbauch positionieren.

Die Schallfeldgeometrie und das Resonanzverhalten der eingesetzten Küvette wird von APPEL [83] analytisch diskutiert und durch Hydrophonmessungen bestätigt. Auf diese Ergebnisse aufbauend wurde für die hier gezeigte Simulation ein

3 Verfahren zur Bestimmung eines globalen Optimums eines komplexen Problems bei dem durch die Verschiebung der Akzeptanzschwelle verhindert werden soll, dass sich die Optimierung frühzeitig in einem Suboptimum festfrisst.

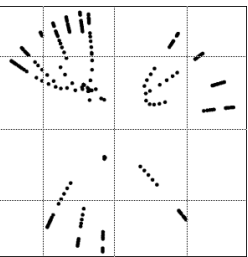




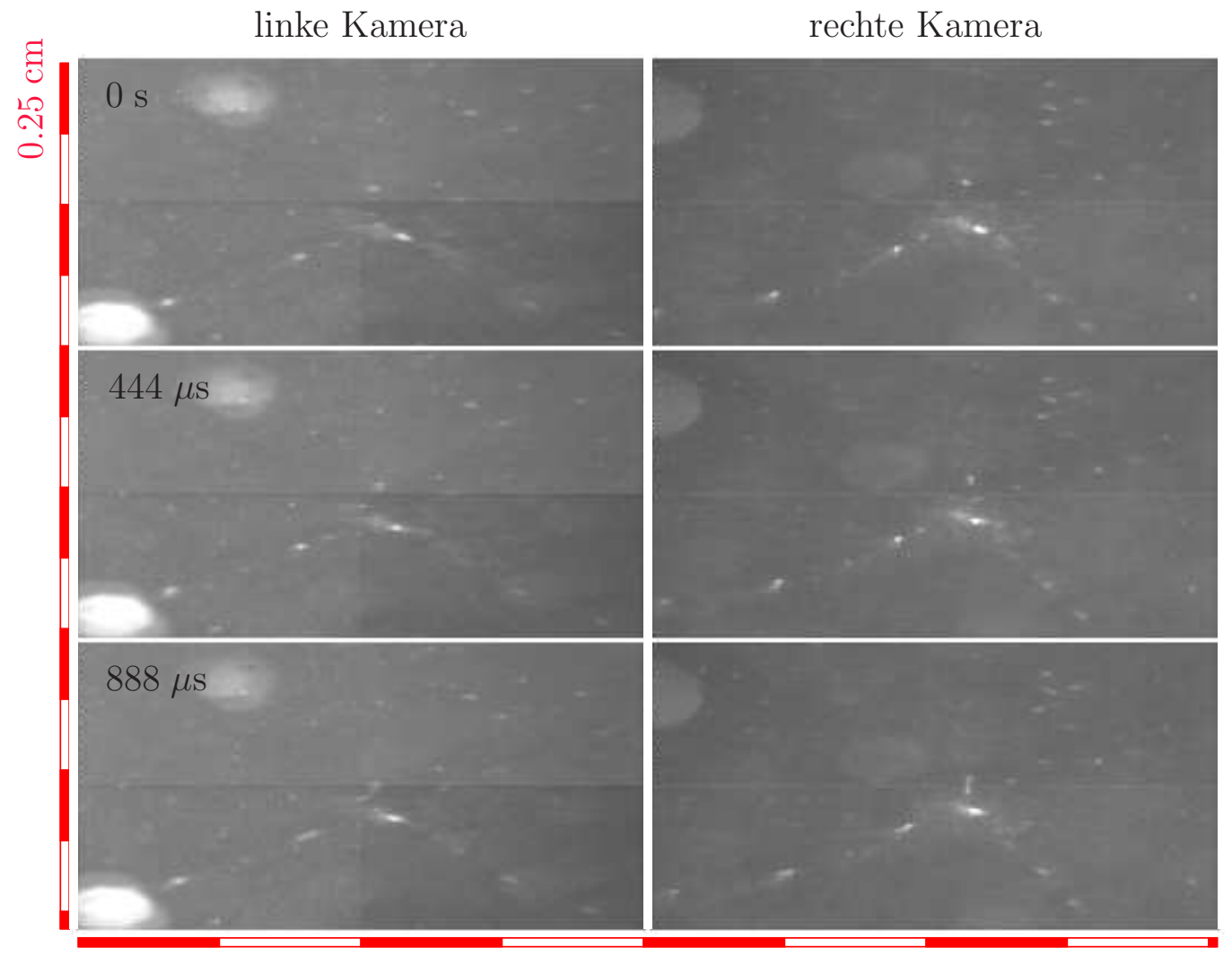

$0.25 \mathrm{~cm}$

Abbildung 5.15: Ausschnitt einer stereoskopische Hochgeschwindigkeitsaufnahme von ApPeL et al. [89]. Zu erkennen sind einzelne Blasen die entlang der Streamerarme in das Zentrum der filamentartigen Struktur laufen; Aufnahmerate 2250 Bilder/s.

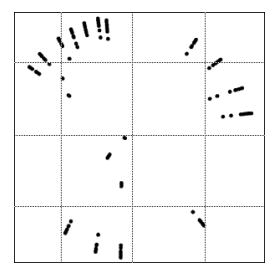




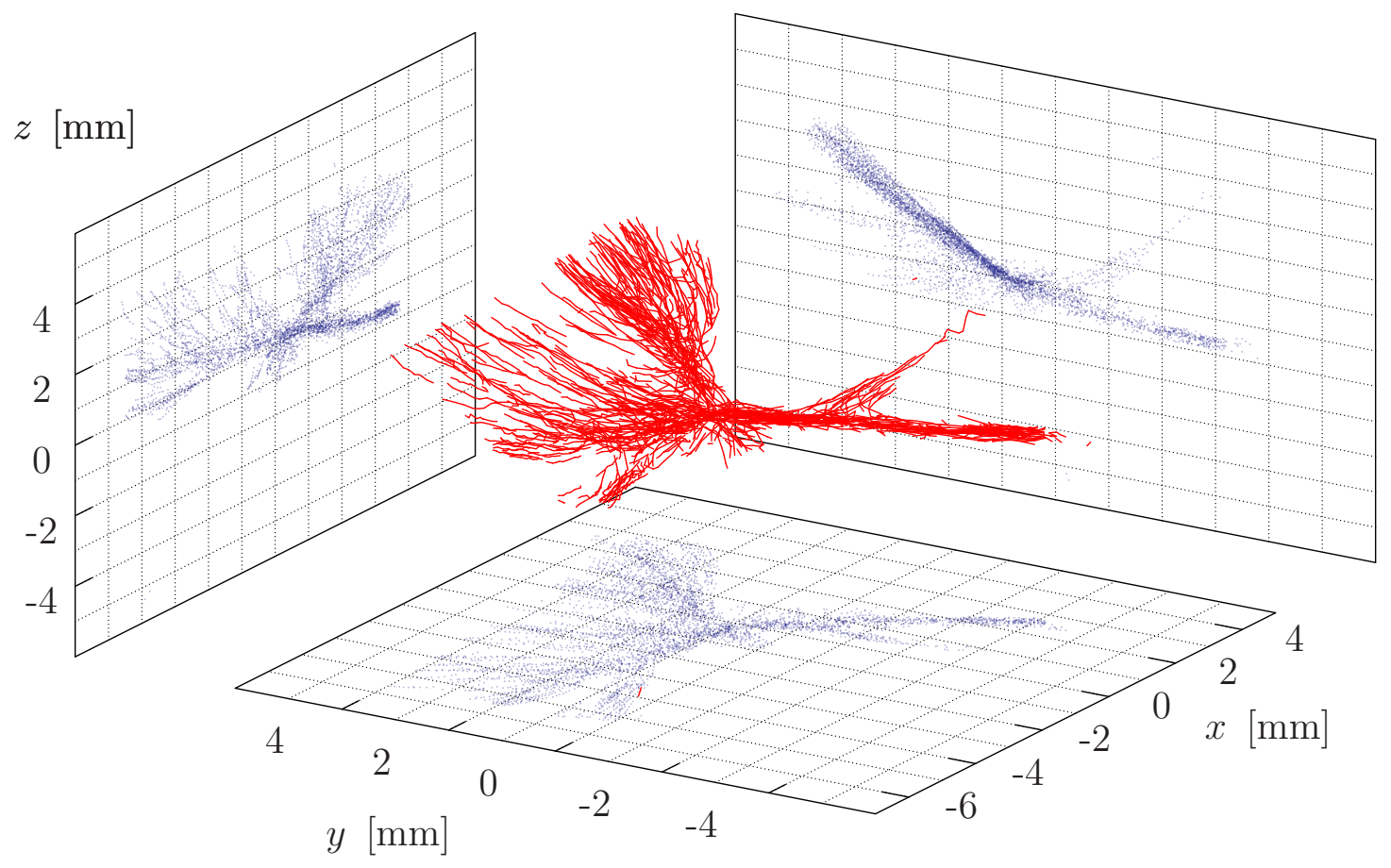

Abbildung 5.16: Darstellung aller detektierten Blasentrajektorien der dreidimensionalen experimentellen Streamerfigur. Aufnahme und Rekonstruktion von AppeL et al. [89]; Aufnahmedauer $7.28 \mathrm{~s}, \nu=23.621 \mathrm{kHz}$, Aufnahmerate 2250 Bilder $/ \mathrm{s}, \hat{p}_{\mathrm{A}} \approx 160 \ldots 210 \mathrm{kPa}$.

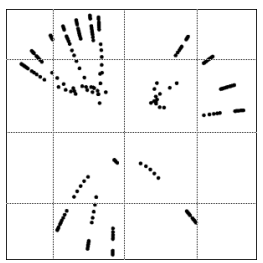


analytisches Stehwellenfeld generiert. Der Schwerpunkt der Streamerfigur wurde dabei an der Position des Druckbauchs ausgerichtet.

\subsubsection{Partikelmodellierung}

Als Startpositionen der in die numerische Simulation eingegangenen Blasen wurden die jeweils ersten räumlichen und zeitlichen Positionen der in Abbildung 5.16 gezeigten rekonstruierten Trajektorien eingesetzt.

$\mathrm{Zu}$ ihren Startzeitpunkten werden alle Blasen mit einem Ruheradius von $R_{0}=$ $R_{\mathrm{n}}(t=0)=5 \mu \mathrm{m}$ initialisiert. Dieser aus anderen Experimenten bestimmte Wert wurde benutzt, da aus den Streulichtaufnahmen keine Ruheradien abgeleitet werden konnten.

Das Resultat der Partikelmodellierung ist im Vergleich zu den rekonstruierten Blasentrajektorien in Abbildung 5.17 dargestellt. Betrachtet wird ein Ausschnitt von 1.5 ms Länge aus der in Abbildung 5.16 gezeigten Figur. Diese Simulation geht von einem analytisch angegebenen Stehwellenfeld der (1, 1, 1)-Mode aus (siehe auch KocH et al. [91, 56]). Die gerichtete Diffusion wird vernachlässigt, Blasen wachsen in dieser Modellierung lediglich durch Kollisionen miteinander an. Die Diffusion fällt für die Volumenänderung von Kavitationsblasen erst auf Zeitskalen ins Gewicht, die deutlich länger sind, als die typische Lebensdauer von Blasen in Streamerfiguren. Es zeigt sich wiederum eine relativ gute Übereinstimmung von gemessenen und simulierten Blasentrajektorien.

Für die von Appel et al. [89] gemessene Vielblasenstruktur in Abbildung 5.16 zeigt Abbildung 5.18 die Geschwindigkeitsverteilungen ${ }^{4}$. Die Blasen der Simulation sind mit ihrer Geschwindigkeitsverteilung in Abbildung 5.18 ebenfalls dargestellt (blau). Sie weisen im Mittel etwa 2 . . 3 mal höhere Geschwindigkeiten auf.

In der mathematischen Formulierung des Modells wurden Annahmen und Näherungen eingesetzt, die zu Ungenauigkeiten des Modells führen, diese erscheinen in dieser Simulation als maßgeblich für die Abweichung in der Geschwindigkeitsverteilung.

${ }^{4}$ Es ist zu vermuten, dass die Abnahme der relativen Häufigkeit hin zu niedrigen Geschwindigkeiten bedingt ist durch die Auswertung der Bilddaten. Liegt die von den Blasen zurückgelegte Distanz unterhalb der Aufösungsgrenze so kann der Algorithmus die Blase in aufeinanderfolgenden Aufnahmen nicht als bewegtes Objekt erkennen und entsprechend nicht von Artefakten in der Aufnahme unterscheiden.

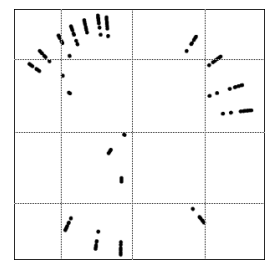




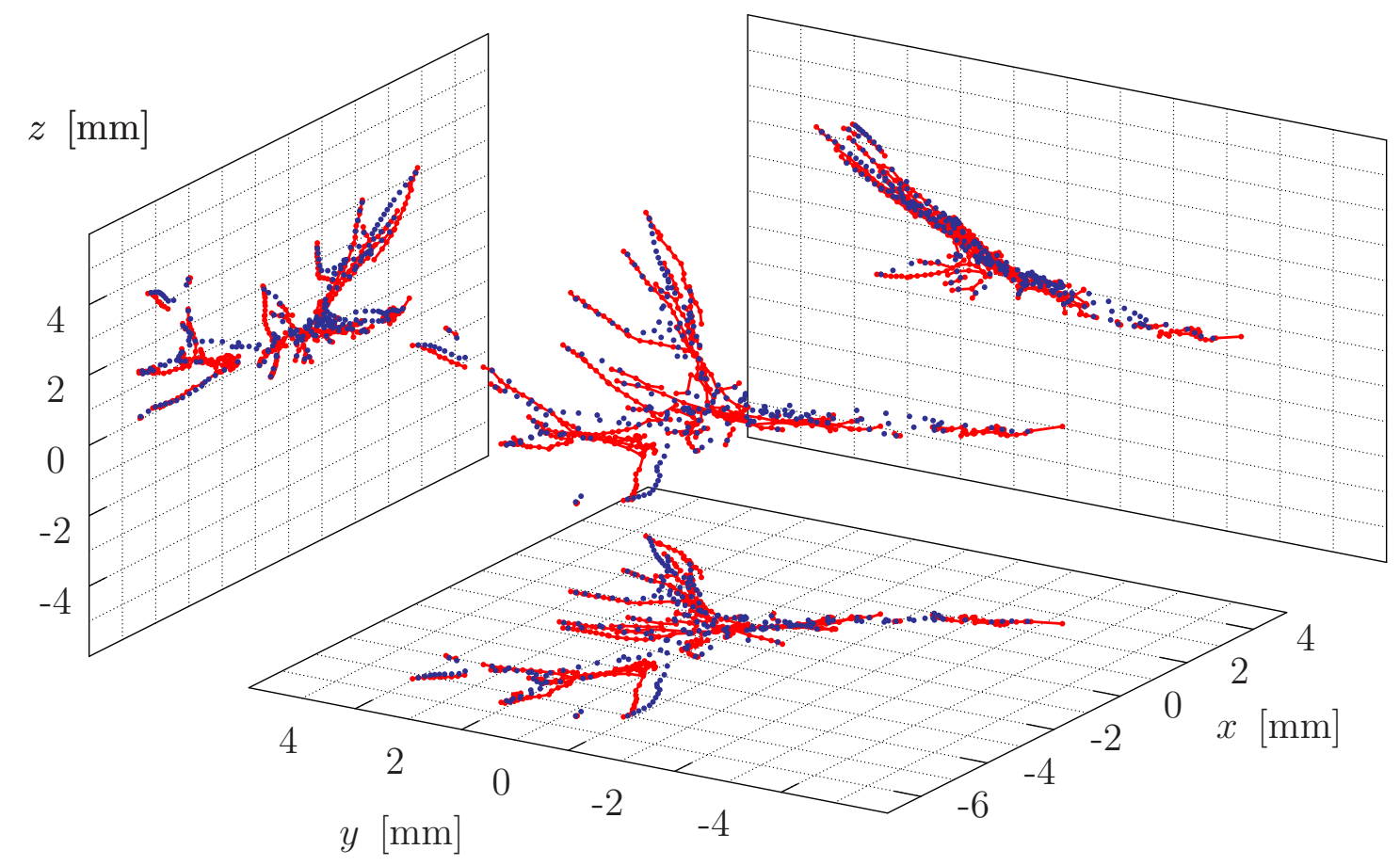

Abbildung 5.17: Gezeigt ist ein Ausschnitt über $1.5 \mathrm{~ms}$ der Blasentrajektorien (rot) der Figur aus Abbildung 5.16. Die überlagerten gepunkteten Trajektorien (blau) stellen das entsprechende Ergebnis der Partikelmodellierung dar, der Punktabstand beträgt $50 \mu \mathrm{s} ; \nu=20 \mathrm{kHz}, R_{\mathrm{n}}=5 \mu \mathrm{m}$, $\hat{p}_{\mathrm{A}}=150 \mathrm{kPa}$.

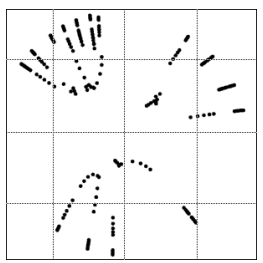




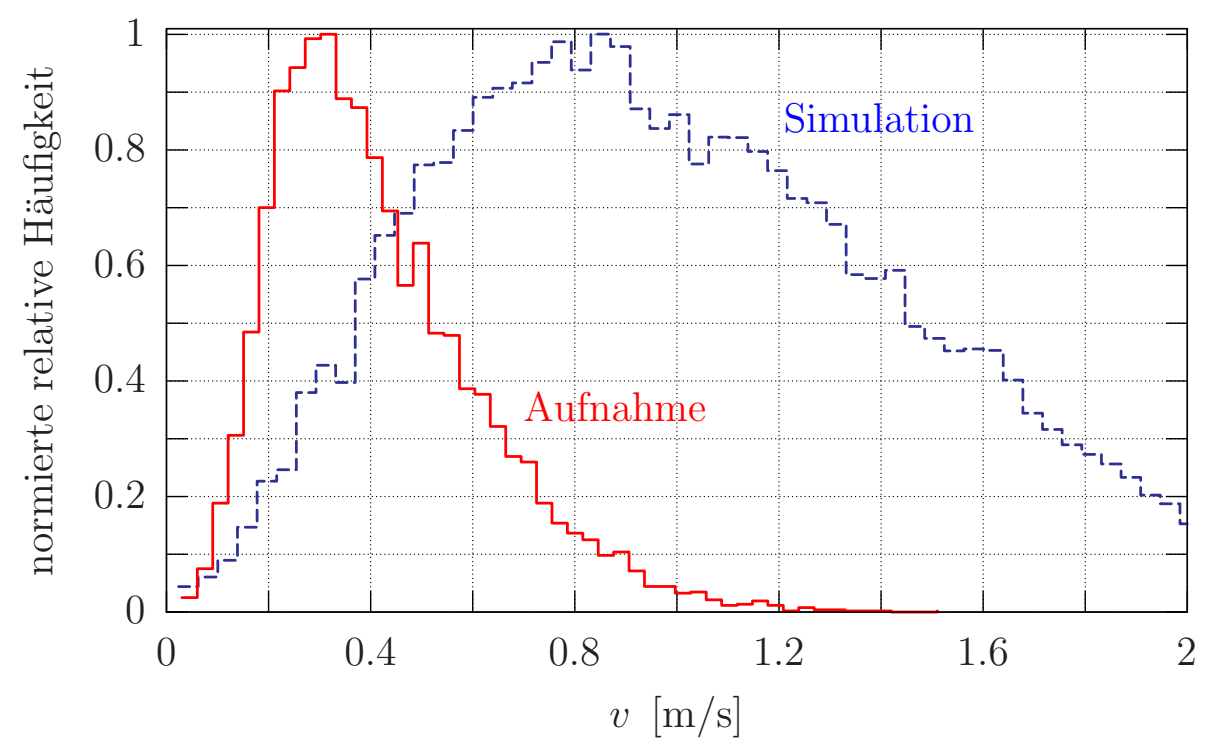

Abbildung 5.18: Geschwindigkeitsverteilungen (rot) der filamentartigen Strukturen aus Abbildung 5.16. Gegenübergestellt die Geschwindigkeitsverteilung der Partikelsimulation (blau) zur Nachbildung dieser Figur.

So erfahren Blasen ihre stärkste Beschleunigung in der Nahfeldwechselwirkung der sekundären Bjerkneskraft (4.5), die in der Modellierung unphysikalisch mit dem Kehrwert des Abstandsquadrates divergiert. Wird die Kollision zweier Blasen durch die Wahl der numerischen Bedingung noch nicht detektiert, so kommt es nochmals zu einer starken Beschleunigung der Blasen, was die Verschiebung der Geschwindigkeitsverteilung zum Teil bewirken kann.

In den Auftragungen in Abbildung 5.19 erfolgt eine direkte Gegenüberstellung der experimentell beobachteten Trajektorien mit den aus der Partikelsimulation resultierenden Bahnen, qualitativ dargestellt für die drei Raumachsen. Das aus dieser Darstellung ersichtliche Geschwindigkeitsverhalten der berechneten Blasen zeigt vor allem für die $z$-Achse gute Übereinstimmungen mit den experimentellen Aufnahmen.

Auffällig sind die Abweichungen auf der $x$ - und $y$-Achse, diese zeigen sich in den Bereichen um $-3 \ldots-5 \mathrm{~mm}$. Hier liegt eine überhöhte Blasengeschwindigkeit in der Simulation gegenüber dem Experiment vor. Diese Abweichung der beiden in der Aufnahme perspektivisch verzerrten Achsen gegenüber der von beiden Kameras unverzerrt erfasst $z$-Achse könnte von einem systematischen Fehler in der Blasendetektion herrühren. Die spezielle Struktur der erfassten Blasenanordnung kann ebenfalls für die Abweichung verantwortlich sein. Die hier eingesetzte Modellierung führt zu der Ausbildung einer zentralen Blase, die durch einlaufende

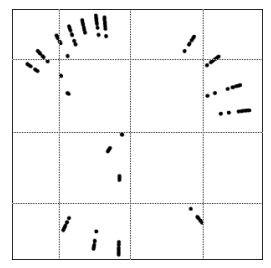



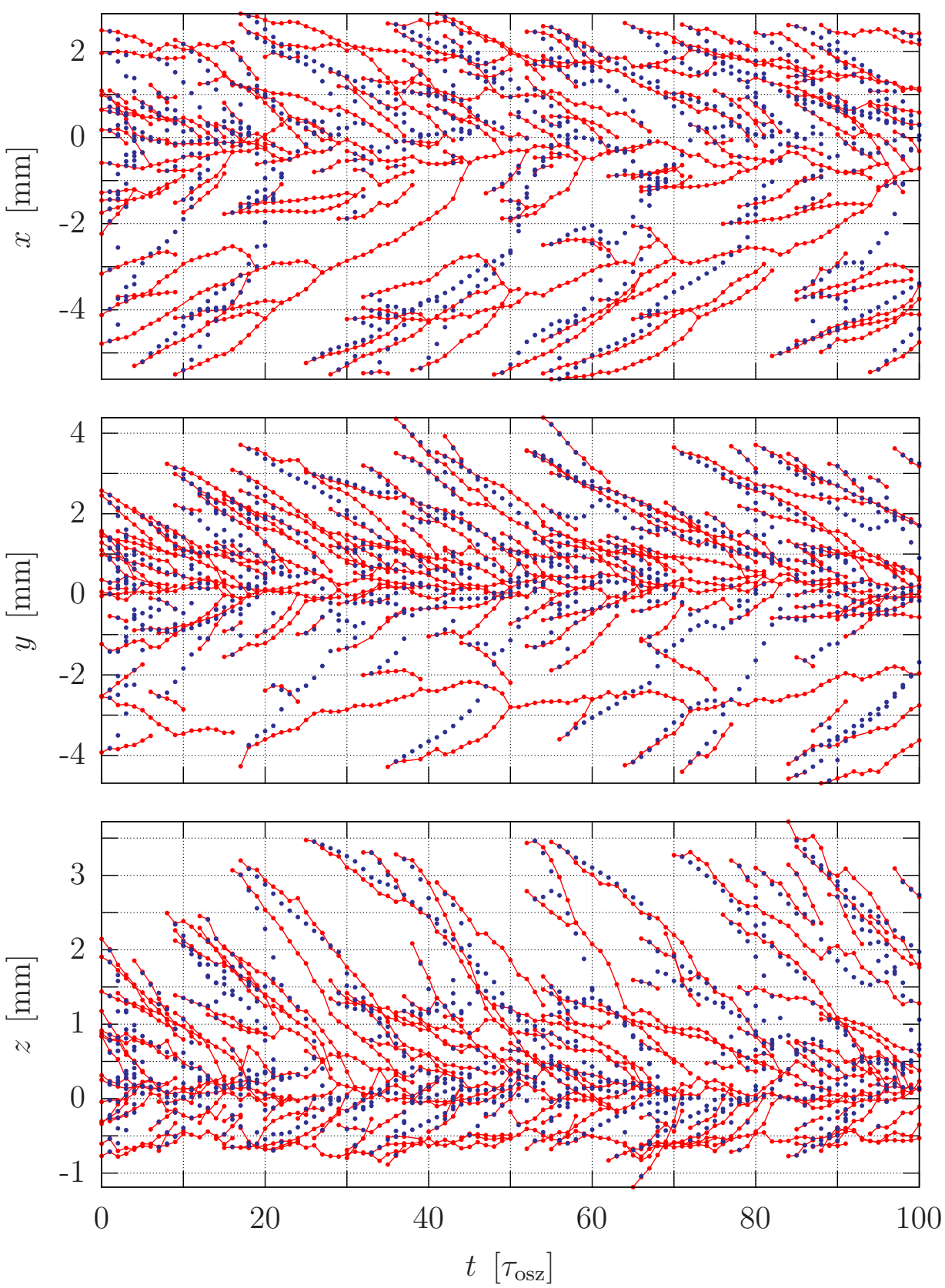

Abbildung 5.19: Darstellung eines Ausschnittes der Blasentrajektorien, der in Abbildung 5.16 gezeigten Struktur (rot) und der zugehörigen Partikelmodellierung (blau). Aufgetragen sind die Raumachsen gegen die Zeit, der Punktabstand beträgt $50 \mu \mathrm{s} ; \tau_{\mathrm{osz}}=50 \mu \mathrm{s}, R_{0}=5 \mu \mathrm{m}, \hat{p}_{\mathrm{A}}=150 \mathrm{kPa}$. 
Streamerarme gespeist wird. Das heißt, es kann keine im Zentralbereich großflächigere Blasenanordnung nachgebildet werden.

Einen stärkeren Einfluss auf die Simulation nimmt zum Einen die Wahl der Ruheradien, die hier einheitlich mit $5 \mu \mathrm{m}$ geschätzt wurden. Zum Anderen liegt der Modellierung die Annahme einer idealen Schalldruckmode zugrunde. In der Realität wird gerade die Blasenkonzentration im Zentrum (Druckbauch) zu einer Schwächung des Schalldrucks führen.

Die deutlich glatteren Blasenbahnen der Simulation gegenüber dem Experiment, lassen darauf schließen, das es zu deutlich mehr Wechselwirkungen mit anderen Blasen kommt, die in den Aufnahmen nicht aufgelöst werden konnten.

Wie in Abschnitt 5.1 diskutiert, besteht eine empfindlichen Abhängigkeit einzelner Blasenbahnen von Parametern wie dem Reibungskoeffizienten, der Schalldruckamplitude, der Diffusion sowie dem abgeschätzten Ruheradius. Aufgrund der relativ häufigen Blasenkollisionen kann in der hier verwendeten Simulation die Diffusion vernachlässigt werden.

Verwendet wurde in dieser Rechnung ein analytisch vorgegebenes Schallfeld der $(1,1,1)-M o d e$, in dem es zu keiner Rückwirkung der Blasen auf die Schallfeldmode kommt.

So lassen sich qualitative Übereinstimmungen mit diesem bisherigen Modellierungsansatz erzielen, jedoch liegen quantitative Abweichungen vor, die die in dieser Arbeit gezeigten weitergehenden Betrachtungen der verschiedenen Effekte erforderlich machen.

Neben der nicht exakt bestimmbaren Schalldruckamplitude liegt die experimentelle Herausforderung in der weiteren Bestimmung von Parametern, etwa dem Gasgehalt des Wassers und der Geometrie der Schallfeldverteilung. Die vorliegende Arbeit stellt mit der Betrachtung der Diffusion in Abschnitt 2.5 oder der Kalkulation möglicher Schallfeldstrukturen in Kapitel 3 numerische Eingrenzungen der Parameterbereiche vor. Speziell mit der Problematik, die an dieser Stelle nicht exakt bestimmbaren Ruheradien zu erhalten, beschäftigt sich der folgende Abschnitt 5.4.

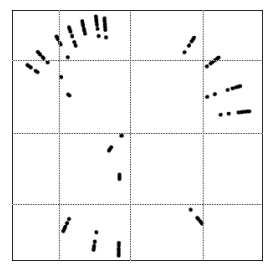




\subsection{Erfassung der Blasenradien}

Für die Erfassung der Blasenradien wird in dem in Abschnitt 5.3 eingesetzten Aufbau, wie ihn Abbildung 5.14 zeigt, eine hochauflösende Kamera mit einem LED-Blitz als Beleuchtung hinzugefügt. Abbildung 5.20 zeigt diesen Aufbau, wie er von APPEL [83] eingesetzt wurde.

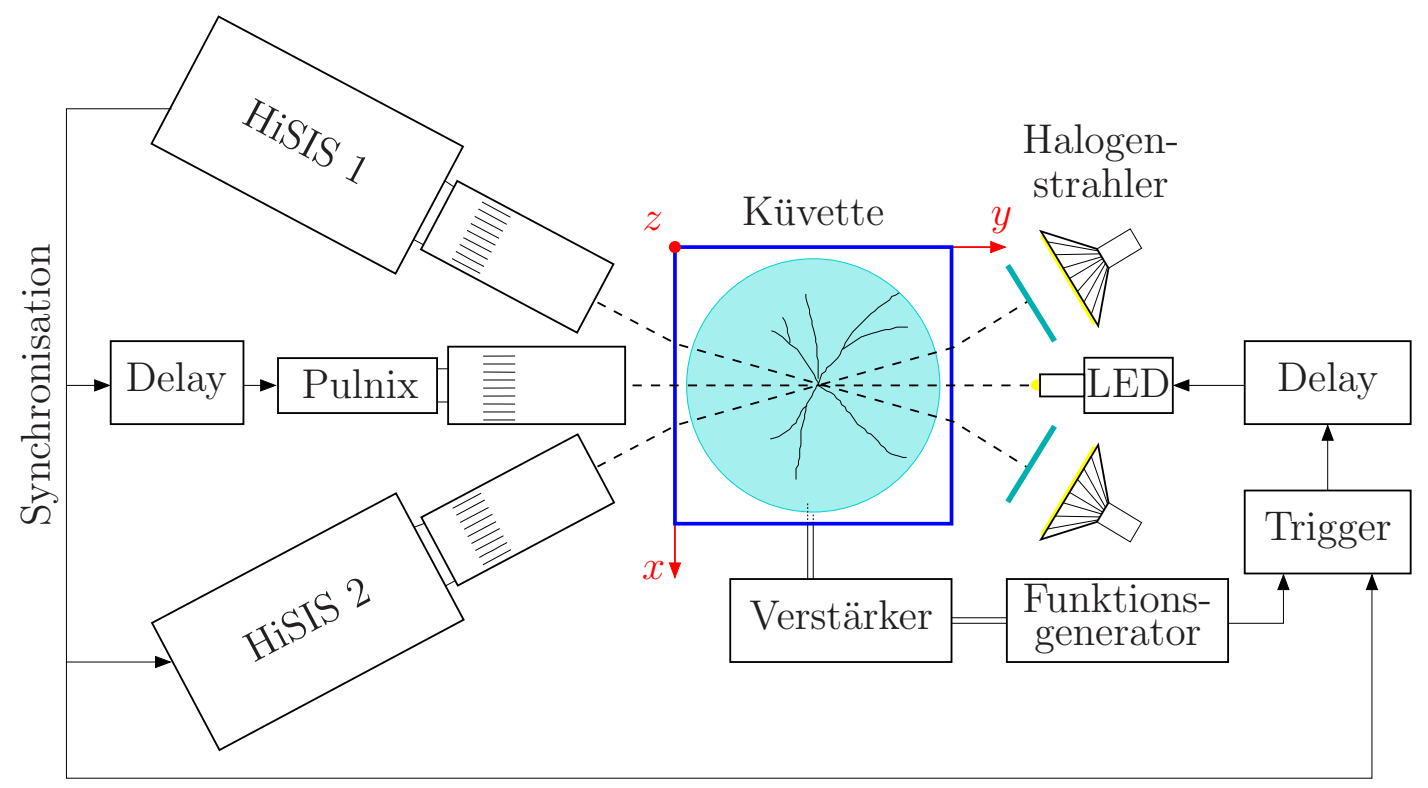

Abbildung 5.20: Schematische Aufbauskizze für stereoskopische Hochgeschwindigkeitsaufnahmen. Aufbau analog zu dem in Abbildung 5.14 gezeigten, erweitert um eine hochaufösende Kamera zur Erfassung der Blasenradien.

Die Phasenlage des Schallfeldes wird so an die Belichtung der Kameras gekoppelt, dass die Blasen zum Aufnahmezeitpunkt möglichst auf ihren Maximalradius aufgezogen sind, das heißt sie können optisch gut aufgelöst werden. Experimentell ist eine Triggerung auf die vergleichsweise lange Phase, die die Blase aufgezogen wird, leichter zu realisieren. Über den durch die Keller-Miksis-Gleichung (2.10) definierten maximalen Radius wird auf den Ruheradius zurückgeschlossen. Durch den bekannten Schalldruck am Ort der Blase werden die radialen Blasenoszillationen für unterschiedliche Ruheradien betrachtet und der Ruheradius der Schwingung angenommen, deren Maximalradius dem beobachteten am nächsten kommt.

In einer Erweiterung des experimentellen Aufbaus kommt eine zusätzliche PulnixKamera zum Einsatz, die durch ihre höhere Aufösung eine genauere Radienbestimmung ermöglicht. Allerdings wird diese Auflösung mit einer geringeren

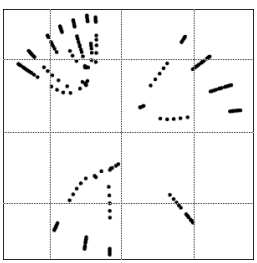


Bildrate und einem verkleinerten Bildausschnitt erkauft, wodurch diese zusätzliche Größeninformation nicht für alle Blasen vorliegt.

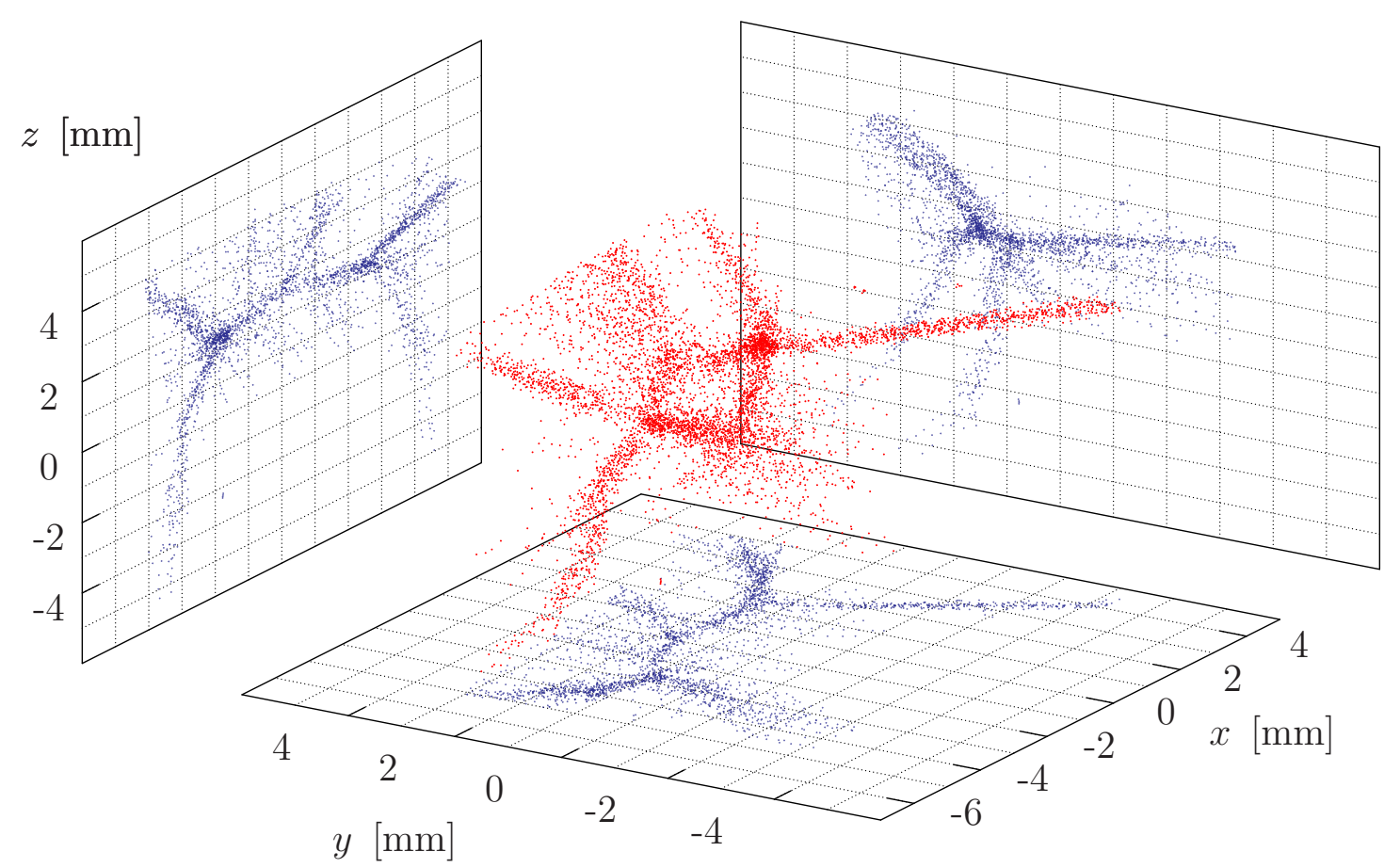

Abbildung 5.21: Darstellung der erkannten Blasenpositionen als Punktwolke im Dreidimensionalen mit Projektionen auf die Flächen; Messung und Tracking von ApPeL et al. [83, 90]; $\nu=22.881 \mathrm{kHz}, \hat{p}_{\mathrm{A}}=132 \mathrm{kPa}$.

Die in Kapitel 2 eingeführte radiale Blasendynamik ist vor allem abhängig von der Schalldruckamplitude am Ort der Blase sowie von ihrem Ruheradius, so dass die Blase abhängig von diesen Parametern ihren maximalen Radius zu unterschiedlichen Phasen des Schallfeldes erreicht. Das heißt, es ist nicht möglich, auf einer Aufnahme alle Blasen in ihrem maximalen Radius zu erfassen.

Für die weitere Rückrechnung bedarf es des Zusammenhangs zwischen maximalem Radius und Ruheradius. Diese Auftragung entspricht gerade der Darstellung der Blake-Schwelle in Abbildung 2.6. In Abbildung 5.22 sind exemplarisch die Kurvenverläufe für zwei Schalldrücke in der Parameterebene von Maximalradius gegen den Ruheradius aufgetragen.

Die in Abbildung 5.23 gegebene Verteilung der Maximalradien kann mit Kennt-

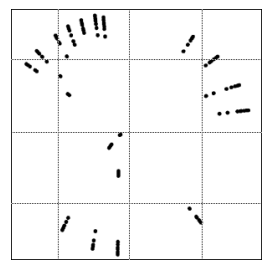




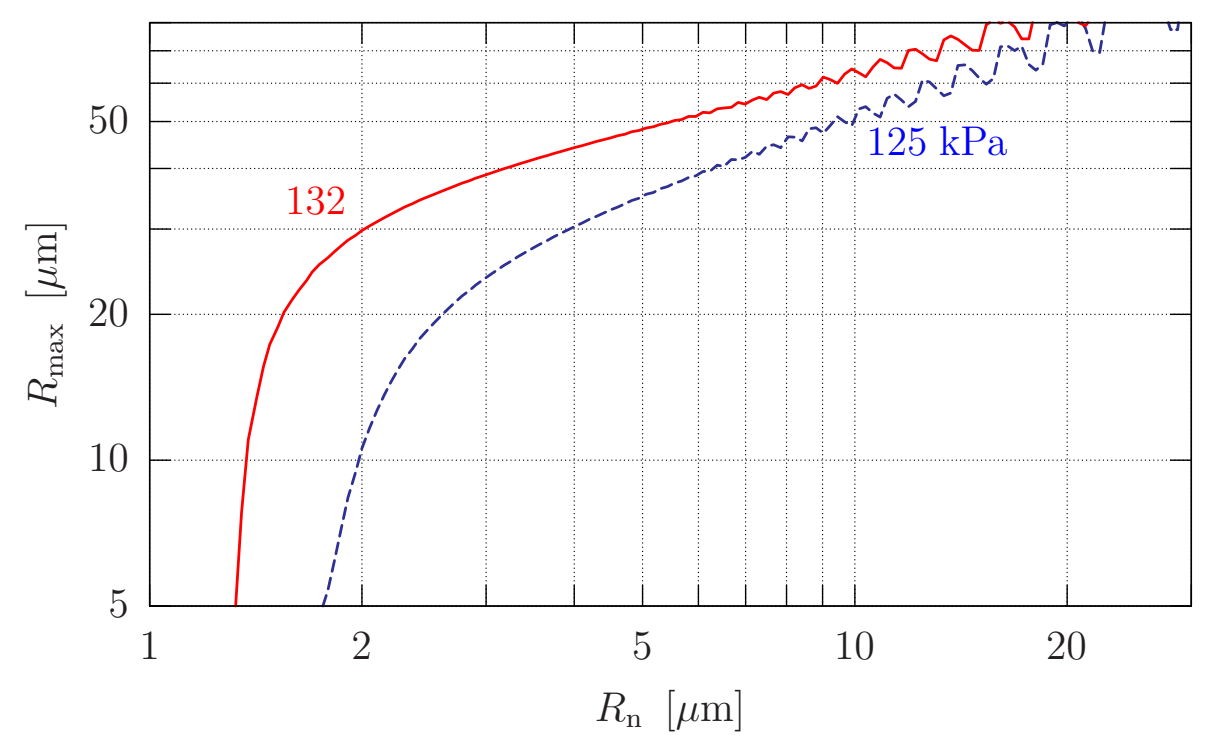

Abbildung 5.22: Darstellung der Kurvenscharen in der doppeltlogarithmische Auftragung vom Maximalradius gegen den Ruheradius, entsprechend der Auftragung der Blake-Schwelle in Abbildung 2.6. Für die Rückrechnung ist jeweils der dem Druck an der Blasenposition entsprechende Kurvenverlauf zu wählen; $p_{\mathrm{A}}=125,132 \mathrm{kPa}, \nu=22.881 \mathrm{kHz}$.

nis der Schalldruckamplitude am Ort der jeweiligen Blase über den in Abbildung 5.22 gegebenen Zusammenhang auf den Ruheradius rückgerechnet werden. Die maximale Schalldruckamplitude ist mit $\hat{p}_{\mathrm{A}}=132 \mathrm{kPa}$ angegeben. Aufgrund der hochaufgelösten Aufnahmen, die zur Radienbestimmung herangezogen werden, ist nur ein Teil der eigentlichen Streamerfigur erfasst. Das bedeutet, dass die Blasen, deren Radien in Abbildung 5.23 erscheinen, sich innerhalb eines Gebietes von $\pm 5 \mathrm{~mm}$ um den Schalldruckbauch der $(1,1,1)$-Mode befinden. Für diese Mode wird gerade davon ausgegangen, dass die Abmessung der Küvette der halben Wellenlänge entspricht:

$$
\lambda_{\mathrm{x}}=2 \cdot d_{\mathrm{x}} \approx 10 \mathrm{~cm}
$$

Bei dieser Wellenlänge bedeutet eine Abweichung um $5 \mathrm{~mm}$ aus dem Druckmaximum einen Druckabfall auf $95 \%$. Nach dieser Abschätzung kann davon ausgegangen werden, dass sich die rückzurechnenden Blasen innerhalb einer Druckamplitude von $p_{\mathrm{A}}=125 \ldots 132 \mathrm{kPa}$ befinden.

Für diese Abgrenzung ist die Rückrechnung auf die Ruheradienverteilung in Abbildung 5.24 gezeigt. Der Schwerpunkt der sich ergebenden Radienverteilung liegt unterhalb von $5 \mu \mathrm{m}$. Oberhalb von $7 \mu \mathrm{m}$ treten deutlich weniger Blasen auf. Diese Gewichtung verstärkt sich mit höheren Schalldrücken. Im Rahmen der Messge-

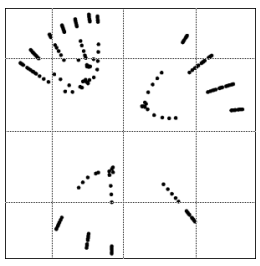




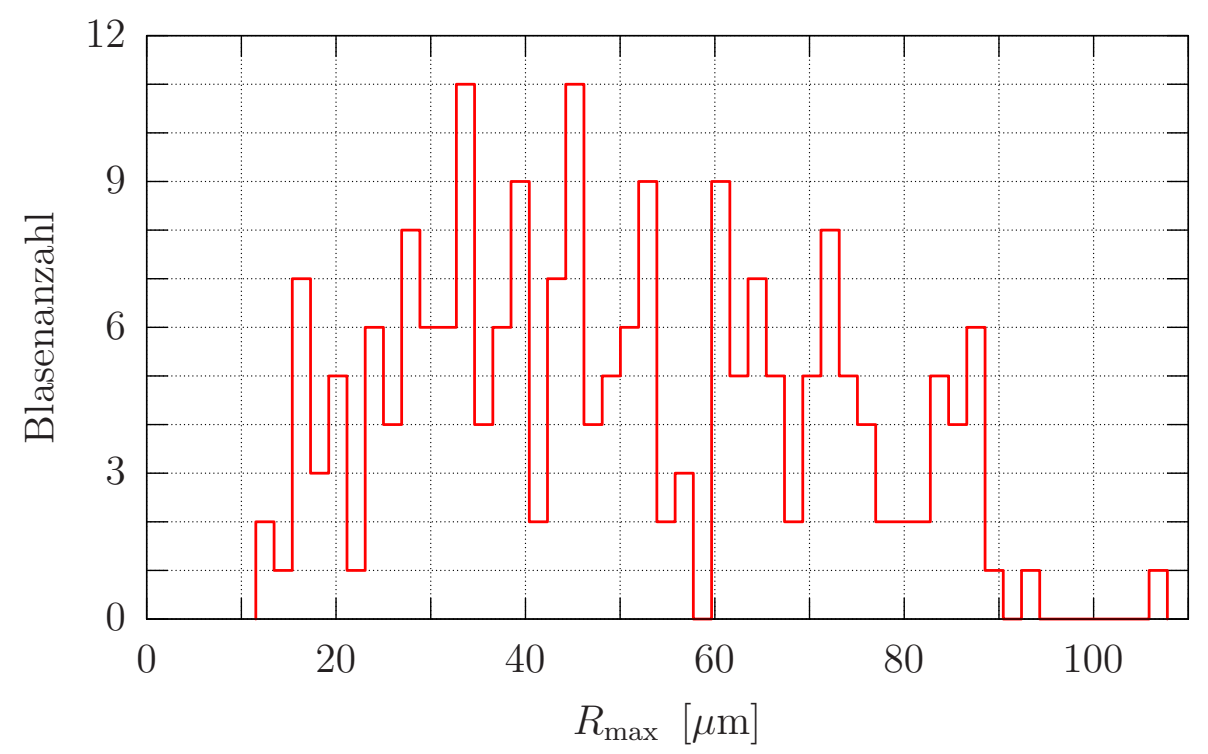

Abbildung 5.23: Blasenradienverteilung der in Abbildung 5.21 dargestellten Vielblasenfigur. Die hier verwendeten Kameras ermöglichen lediglich eine Radiusbestimmung von etwa jeder tausendsten der in den Hochgeschwindigkeitsaufnahmen erfassten Blasen.

nauigkeit, vor allem was die Erfassung der Maximalradien und die Angabe der Schalldruckamplitude betrifft, bestärkt dieses Resultat die in bisherigen Arbeiten angenommenen Ruheradienverteilung um $5 \mu \mathrm{m}$.

Die hier vorgestellte Bestimmung der Ruheradien weist für die praktische Nutzung den wesentlichen Nachteil auf, dass für eine exakte Rückrechnung aus dem maximalen Blasenradius der Schalldruck am Ort der Blase bekannt sein muss. Außerdem ist mit den hier verwendeten Kameras aufgrund der Bildaufnahmeraten sowie der Größe der Bildausschnitte eine räumlich hochaufgelöste Erfassung lediglich von etwa jeder tausendsten Blase aus der Hochgeschwindigkeitsaufnahme möglich. Alternativ bietet sich an, die sich aus der theoretischen Betrachtung ergebenden in Abbildung $4.6 \mathrm{zu}$ sehenden Akkumulationsgebiete von Blasen in der Parameterebene von Schalldruck und Ruheradius heranzuziehen, um die zu erwartenden Ruheradien einzugrenzen.

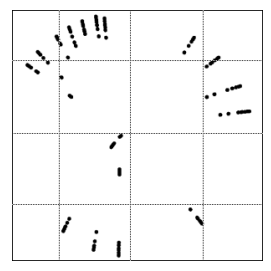




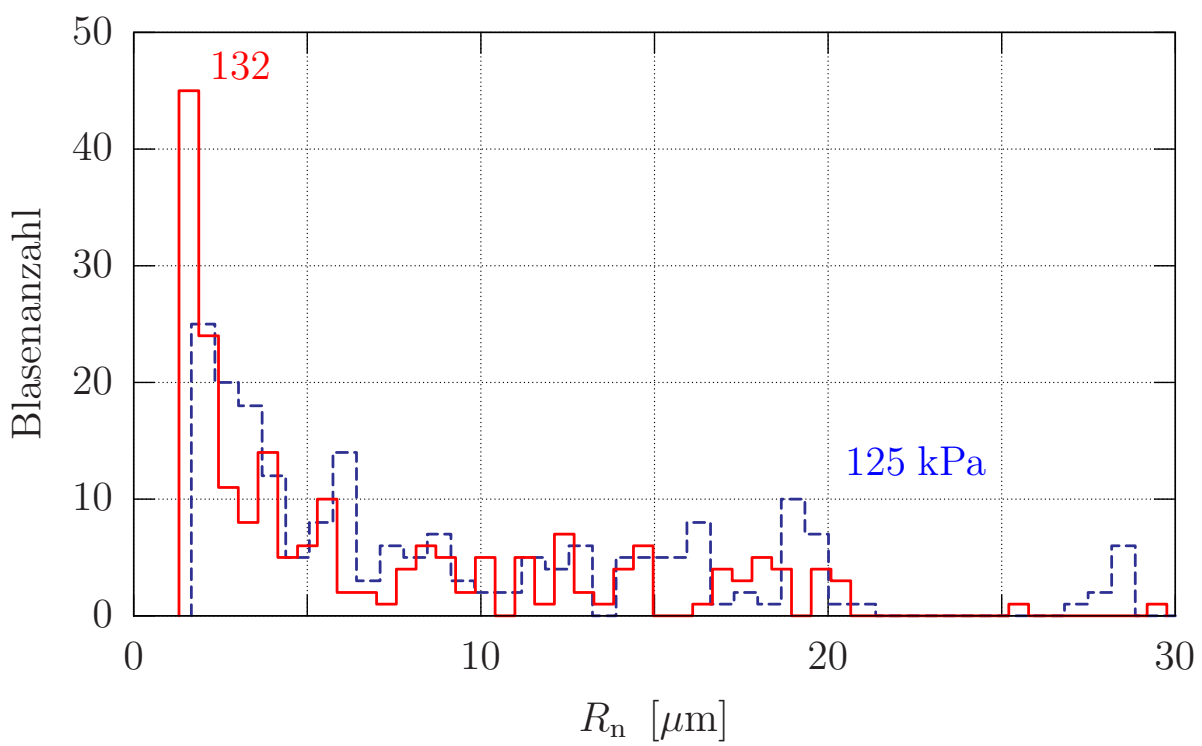

Abbildung 5.24: Verteilung der rückgerechneten Ruheradien aus der in Abbildung 5.23 gezeigten Verteilung der Maximalradien über den Zusammenhang von Maximal- zu Ruheradius in Abbildung 5.22.

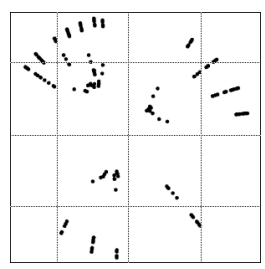




\title{
5.5 Doppelschichtige Blasenfigur
}

Abbildung 5.25 zeigt die im Stehwellenfeld auftretenden von METTiN et al. $[92,84]$ beschriebenen doppelschichtigen Blasenfiguren. Die Aufnahme wurde in dem im Anhang B beschriebenen Ultraschallreaktor der Firma ELAC gemacht. Weitgehend unabhängig von der eingekoppelten Leistung ist die paarweise Ausbildung schichtartiger Filamentstrukturen zu sehen.

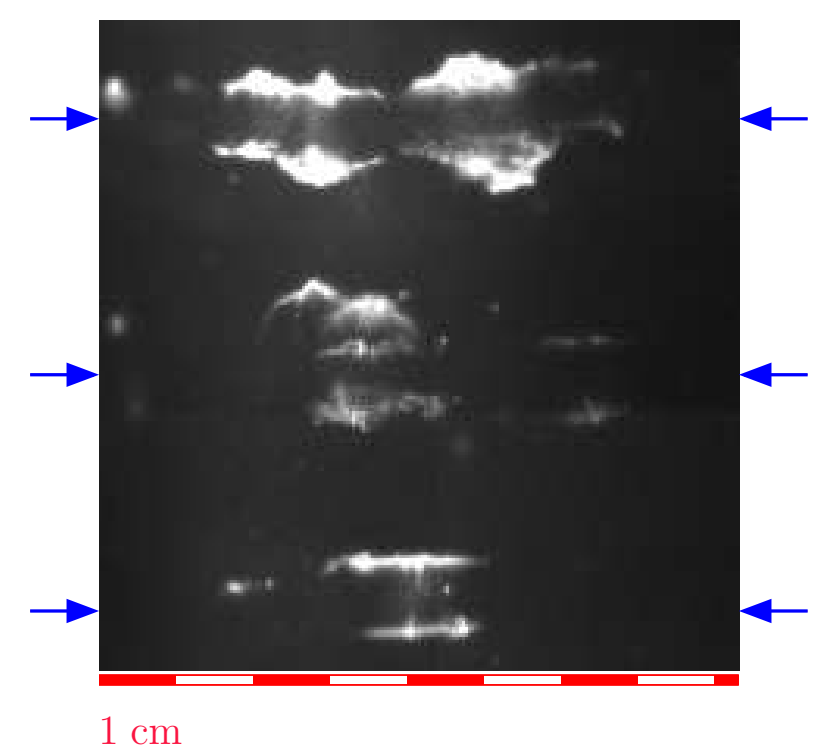

\begin{abstract}
Abbildung 5.25: Anordnung doppelschichtiger Blasenstrukturen über einen Raumbereich mehrerer Wellenlängen. Vertikal bilden sich regelmäßige Schichten um Druckknotenebenen aus (Pfeile); in der Horizontalen sind die Strukturen beweglicher. Aufnahme von Krefting [19]; $\nu=40 \mathrm{kHz}$.
\end{abstract}

Die regelmäßige Anordnung lässt sich in Verbindung mit dem in dem Reaktor ausgebildeten Stehwellenfeld deuten. Wie in Abbildung 5.26 skizziert, sind die Druckbauchebenen weitgehend blasenfrei. Die Blasenstrukturen ordnen sich vorrangig auf der vertikalen Achse paarweise um die Druckknoten an. Horizontal bewegen sie sich freier, so dass ganze Doppelschichtfiguren mit benachbarten Figuren verschmelzen oder sich aufspalten können.

An der Wasseroberfläche kommt es zur Formung einzelner filamentartiger Strukturen, deren Position mit leichten Erhebungen der Wasseroberfläche korrelieren.

Die in Abbildung 5.27 gezeigte Hochgeschwindigkeitsaufnahme einer doppelschichtigen Struktur bestätigt die Annahme der Formierung dieser Struktur um einen Druckknoten. Innerhalb einer Schallfeldperiode (hier $25 \mu \mathrm{s}$ ) kommt es zu einem wechselseitigen Aufschwingen beider Schichten der Struktur, was sich durch

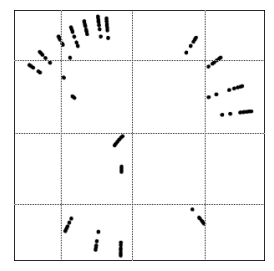




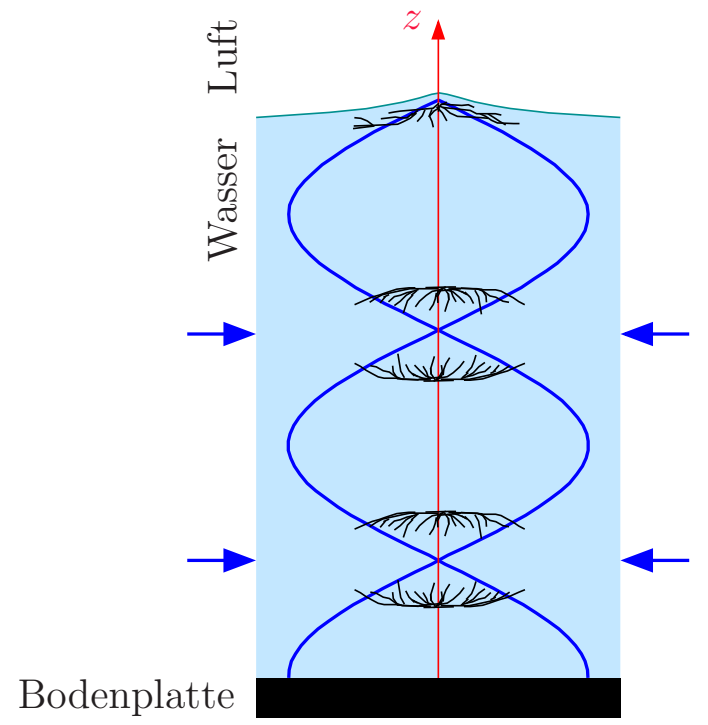

Abbildung 5.26: Schematische Darstellung der ausgebildeten Modenstruktur entlang der $z$-Achse. Um die Druckknoten (Pfeile) können doppelschichtige Figuren entstehen. An der Oberfläche kann eine einzelne sternförmige Blasenanordnung auftreten.

den Phasensprung über dem Schallfeldknoten in dem Stehwellenfeld erklärt ${ }^{5}$.

\subsubsection{Numerische Simulation}

Zur numerischen Betrachtung einer einzelnen Doppelschichtfigur ist es ausreichend, den Raumbereich um einen Schallfeldknoten zwischen zwei Bäuchen herauszugreifen. Zu diesem Zweck wird hier eine rein numerische Modellküvette generiert, deren Resonanzfrequenz in der geforderten Mode gerade bei $25 \mathrm{kHz}$ liegt. Diese Küvette besteht aus einem Quader quadratischer Grundfläche $x \times y$ mit je einer symmetrisch positionierten Fläche eines Ultraschallwandlers an Unter- und Oberseite:

$$
\begin{aligned}
x \text { - und } y \text {-Abmessung: } & 5.5 \mathrm{~cm} \\
z \text {-Abmessung: } & 5.246 \mathrm{~cm}
\end{aligned}
$$

Wandlerdurchmesser: $4.7 \mathrm{~cm}$

Werden die gegenüberliegenden Ultraschallwandlerflächen als schallhart angenommen und die weiteren Grenzflächen als schallweiche Abschlüsse, so ergibt sich für eine Anregung von $\nu=25 \mathrm{kHz}$ die in Abbildung 5.28 gezeigte Eigen-

5 Das Erscheinungsbild dieses wechselseitigen Pulsierens der beiden Schichten hat ihr den Namen „Jellyfish“-Struktur eingebracht.

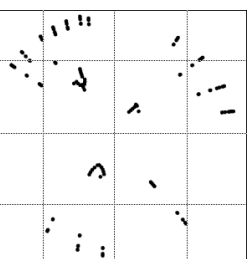




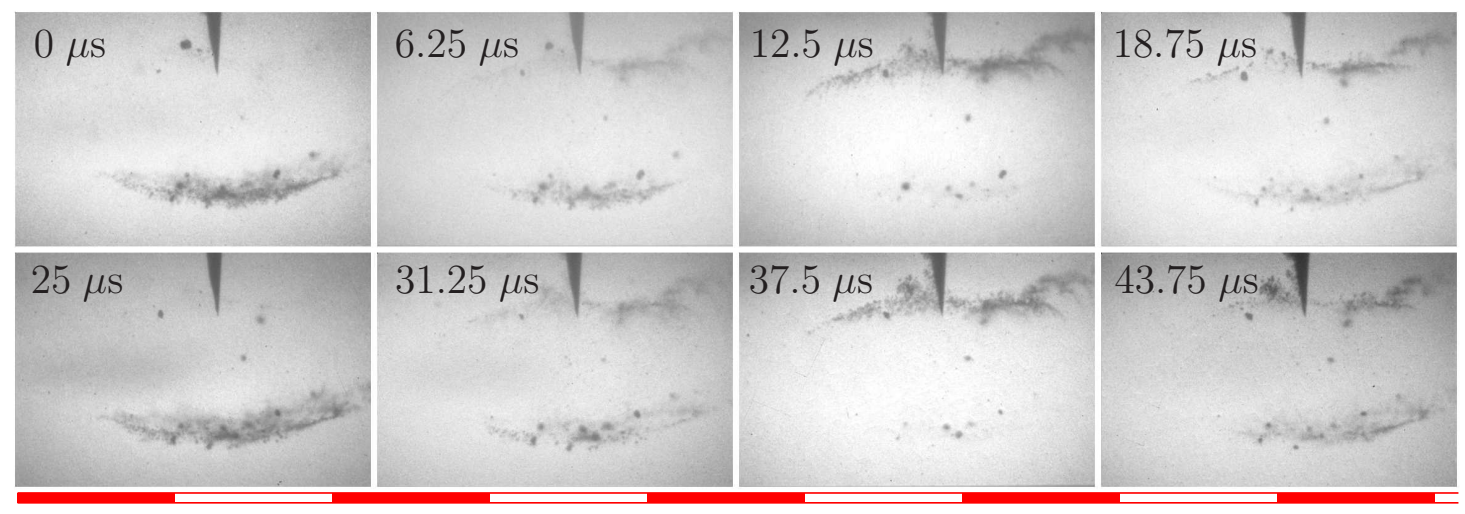

$5 \mathrm{~mm}$

Abbildung 5.27: Hochgeschwindigkeitsaufnahme einer im Stehwellenfeld um einen Druckknoten angeordneten doppelschichtigen Figur; Aufnahme von Mettin et al. [92]; $\nu=40 \mathrm{kHz}$.

mode der Küvette mit einer Knotenfläche in der $x$-y-Ebene bei $z=0$. An dieser Fläche findet gerade der oben diskutierte Phasensprung zwischen 0 und $\pi$ statt.

Die insgesamt 72 Startpositionen der simulierten Blasen liegen bei $z= \pm 1 \mathrm{~mm}$ auf einem Ring von $1 \mathrm{~cm}$ Radius ober- bzw. unterhalb der Knotenfläche. Als Startwert werden Blasen mit einem Ruheradius von $R_{\mathrm{n}}=4 \mu \mathrm{m}$ in die Simulation eingegeben. Es wird ein maximaler Schalldruck von $\hat{p}_{\mathrm{A}}=300 \mathrm{kPa}$ angenommen. Die Initialisierung der Blasen erfolgt in für jeden Punkt zufällig gewählten zeitlichen Abständen von dem 100 bis 300fachen der Schallfeldperiode $T=1 / \nu=40 \mu \mathrm{s}$. Während des Laufs der Simulation über 10000 Schallfeldperioden (entspricht $0.4 \mathrm{~s}$ ) treten bis zu 600 Blasen zeitgleich auf.

Die in Abbildung 5.29 (links) abgebildeten Langzeitbelichtungen zeigen eine typische doppelschichtige Blasenfigur im Experiment in Aufsicht und Seitenansicht. In der Aufsicht erscheint die untere Figur im Grauton während die obere weiß abgebildet wird.

Das Ergebnis der Partikelsimulation zeigt Abbildung 5.29 (rechts). Zu erkennen sind die Quellpunkte, von denen in der seitlichen Ansicht ( $x$ - $z$-Ebene) eine „Blasenwand" ausgeht, wie sie in den langzeitbelichteten Aufnahmen auch teilweise sichtbar wird. Die Blasen befinden sich in diesem relativ transparent erscheinenden Schleier nahe dem Druckknoten, werden demnach in ihrer radialen Dynamik nur schwach getrieben. Näher am Druckbauch erreichen sie größere maximale Radien und erscheinen entsprechend deutlicher in den experimentellen Aufnahmen. In der Gleichgewichtslage zwischen dem hier abstoßenden Druckbauch und den Knotenflächen bildet sich schließlich beidseitig der Druckknotenfläche die zu

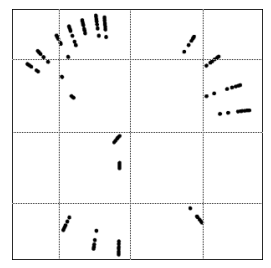




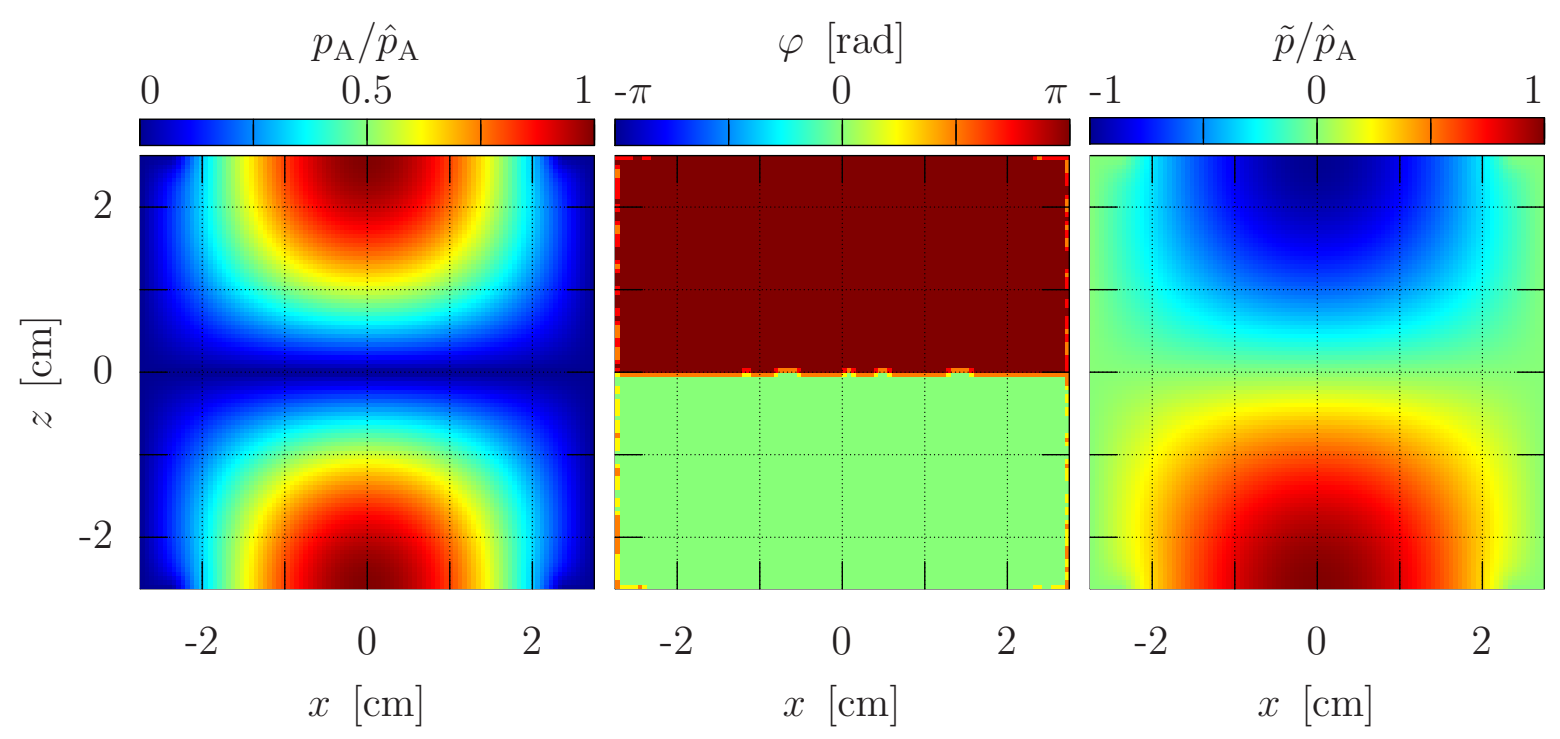

Abbildung 5.28: Auftragung der normierten Schalldruckamplitude $p_{\mathrm{A}} / \hat{p}_{\mathrm{A}}$, der Phasenlage $\varphi$ sowie des Realteils $\tilde{p} / \hat{p}_{\mathrm{A}}$ des stehenden Wellenfeldes als Lösung der Helmholtz-Gleichung (3.3). Randbedingungen entsprechend der Modellküvette, die in der $x$ - $y$-Ebene $(z=0)$ eine Knotenfläche ausbildet; $\nu=25 \mathrm{kHz}$, Kantenlänge $x \times y \times z=5.5 \times 5.5 \times 5.246 \mathrm{~cm}^{3}$.

erkennende dichte Blasenschicht aus.

Durch die Seitenansicht der doppelschichtigen Figur in Abbildung 5.29 (unten) wird eine weitere von der Simulation wiedergegebene Eigenschaft dieser Blasenstruktur sichtbar. Um die Mittelachse $(x=0, y=0)$ kommt es in den beiden sternförmig mit Blasen besetzten Schichten zu „Einstülpungen“, aus denen sich Blasen lösen und in die Mitte der Gesamtstruktur wandern können. Wie es auch in der gezeigten experimentellen Aufnahme zu sehen ist, kann eine relativ große Blase eine stabile Position einnehmen. Die Ablösung von Blasen, die zunächst in Richtung der Knotenfläche wandern, ist ebenfalls in der Simulation zu erkennen. In Abbildung 5.29 (unten) kennzeichnet beispielsweise ein Pfeil solch eine Blase. Aufgrund der implementierten Oberfächen-Stabilitätsgrenze sowie der gerichteten Diffusion liefert die Simulation jedoch keine Blase in einer Größenordnung, die sich dauerhaft im Mittelpunkt der Figur halten könnte.

Die Lage auf der Knotenfläche ist für eine große Blase stabil, dagegen befindet sich eine kleine Blase auf der Knotenfläche in einem instabilen Gleichgewicht. Das heißt, abhängig von einer kleinen Anfangsauslenkung würde die Blase zu einem der Druckbäuche beschleunigt werden. Jedoch kehrt sich wie in Abschnitt 4.2 ausgeführt abhängig von der Schalldruckamplitude und dem Ruheradius der Blasen die Wirkungsrichtung der primären Bjerkneskraft um, so dass auch Bla-

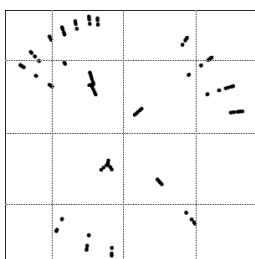


sen vom Druckbauch weg beschleunigt werden können. Für diese Blasen wird eine Lage zwischen Druckknoten und Druckbauch stabil. Wächst eine Blase an, zum Beispiel durch Vereinigungen mit Nachbarblasen, verschiebt sich ihre Gleichgewichtslage zum Druckknoten hin. Dies passiert bevorzugt im Zentrum einer Schicht, was zu den beobachteten Einstülpungen führen kann.

Die überhöht erscheinende Ausdehnung der modellierten doppelschichtigen Figur in Abbildung 5.29 (unten) entlang der $z$-Achse lässt vermuten, dass die hier angenommene Schallfeldgeometrie nicht die genaue Situation in dem UltraschallReaktor wiedergibt oder auch dass der hier angenommene Maximaldruck noch zu niedrig ist. In dieser Betrachtung erfolgt zudem keine Rückwirkung der Blasenkonzentration auf das Schallfeld.

In der Aufsicht in Abbildung 5.29 (oben) ist die sternförmige Struktur zu sehen, die von der Simulation gut nachgebildet wird. Sie zeichnet sich durch eine deutliche Blasenkonzentration um den Mittelpunkt aus. Die von den auf einem Kreis angeordneten Blasenquellen startenden Blasen zeigen in der „langzeitbelichteten“ Darstellung der berechneten Struktur zunächst gerade dem Druckgradienten folgende Bahnen. Im Unterschied zu der experimentellen Betrachtung, in der die Startpunkte in einem immer feineren verästelten Trajektoriengeflecht zu liegen scheinen, starten sie in der Simulation von festen Punkten mit einem relativ großen Ruheradius $R_{\mathrm{n}}=4 \mu \mathrm{m}$, was sie in den Darstellungen deutlich sichtbar macht und die Simulation etwas unnatürlich regelmäßig wirken lässt.

Die Doppelschichtstruktur ist an Oberflächen sehr erosiv tätig, was zum Beispiel in der Ultraschallreinigung eine Rolle spielt. Diese Erosion ist in Abbildung 5.30 anhand von abgereinigter schwarzer Tinte auf einer Glasplatte sichtbar gemacht. Zu erkennen ist eine doppelschichtige Blasenstruktur von der Seite (hell). Die sehr kleinen und hier nicht sichtbaren Blasen zwischen den Schichten haben offenbar keine Erosionsaktivität, da sie nahe des Druckknotens nur eine schwache Anregung und keinen starken Kollaps erfahren. Letzterer findet an Grenzflächen asymmetrisch statt und sorgt über Stoßwellen und Jetströmungen für die Erosion.

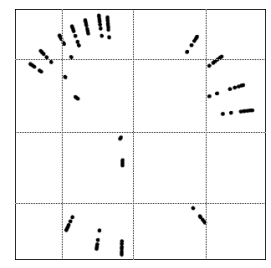




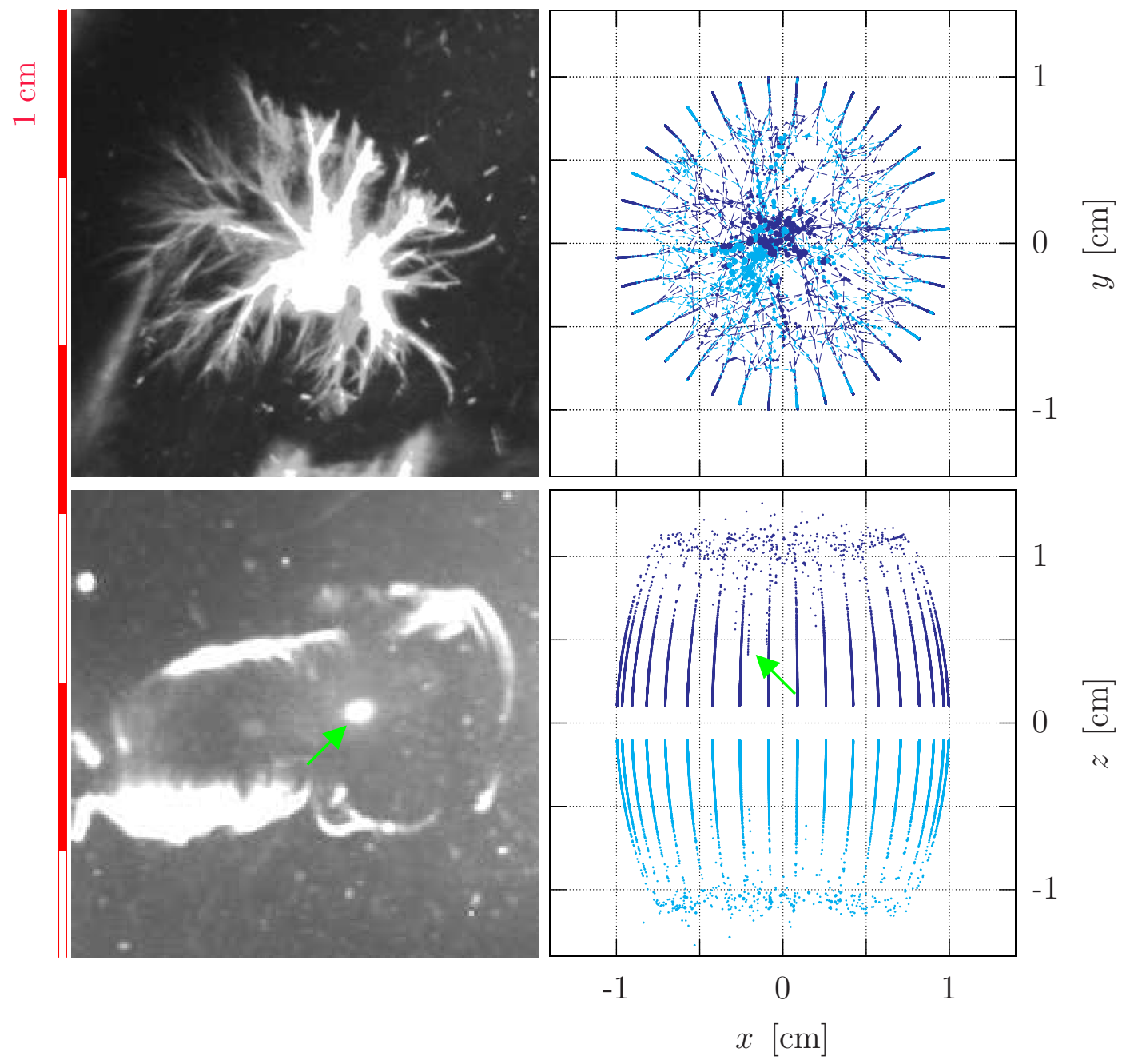

Abbildung 5.29: Gegenüberstellung der doppelschichtigen Blasenstruktur in Experiment und Simulation: Aufsicht (oben) und Seitenansicht (unten). Dargestellt sind „Langzeitbelichtungen“. In der Simulation sind die Blasenpositionen über $0.4 \mathrm{~s}$ gezeigt. Das deutlich symmetrische Ausgehen von den auf einem Kreis angeordneten Startpositionen tritt hier hervor. Die Simulation wurde mit $4 \mu \mathrm{m}$-Blasen initialisiert, die sich nahe den Druckknoten relativ langsam bewegen und somit eine starke Spur zeichnen. In der seitlichen Darstellung (unten) ist im Experiment wie auch in der Simulation eine Blase nahe des Zentrums (Knotenfläche) der Gesamtfigur zu erkennen (Pfeile). Die Punktgröße in der Darstellung skaliert mit dem Blasenruheradius; experimentelle Aufnahmen von MetTin et al. [84]; $\nu=25 \mathrm{kHz}$, Simulation bei $\hat{p}_{\mathrm{A}}=300 \mathrm{kPa}$. 


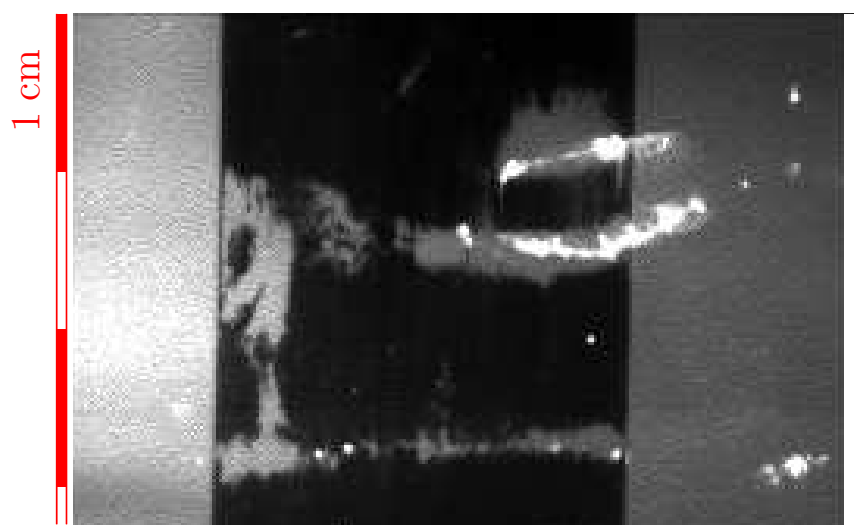

Abbildung 5.30: Zu sehen ist eine doppelschichtige Blasenfigur, die in zwei getrennten Schichten eine Abtragung der mit Filzmarker auf eine Glasplatte aufgetragene Beschichtung bewirkt. Die Einwirkungsdauer der Blasen liegt hier im Sekundenbereich. Aufnahme von Krefting [19]; Leitungswasser, $\nu=40 \mathrm{kHz}$.

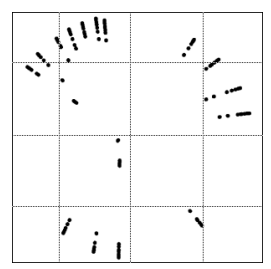




\subsection{Kreuzförmige Streamerfigur}

Durch das gezielte Einbringen von Blasenquellen gelingt es KREFTing [19] eine aus vier Armen bestehende Streamerfigur zu initialisieren.

Eingesetzt ist die aus Abschnitt 3.2 bekannte mit Leitungswasser befüllte HellmaKüvette. Das Resonatorsystem wird bei etwa $24 \mathrm{kHz}$ betrieben, womit sich in der verwendeten Anordnung eine der Abbildung 3.4 (links) entsprechende (1, 1, 1)Mode ausbildet. Die langzeitbelichteten Aufnahmen in Abbildung 5.31 wurden bei einer Bildrate von 60 Bilder/s mit der im Anhang B beschriebenen PulnixKamera bei einer Auflösung von $86.7 \mu \mathrm{m} /$ Pixel gemacht. Durch die Wasseroberfläche wird eine Nadel eingetaucht, an deren Spitze die Blasenbildung begünstigt ist. In der $x$-z-Ebene $(y \approx 0)$ werden weitere Blasenquellen an den Seitenflächen und dem Küvettenboden positioniert und zwar in Form von Luftblasen, die an der Küvettenwand anhaften.

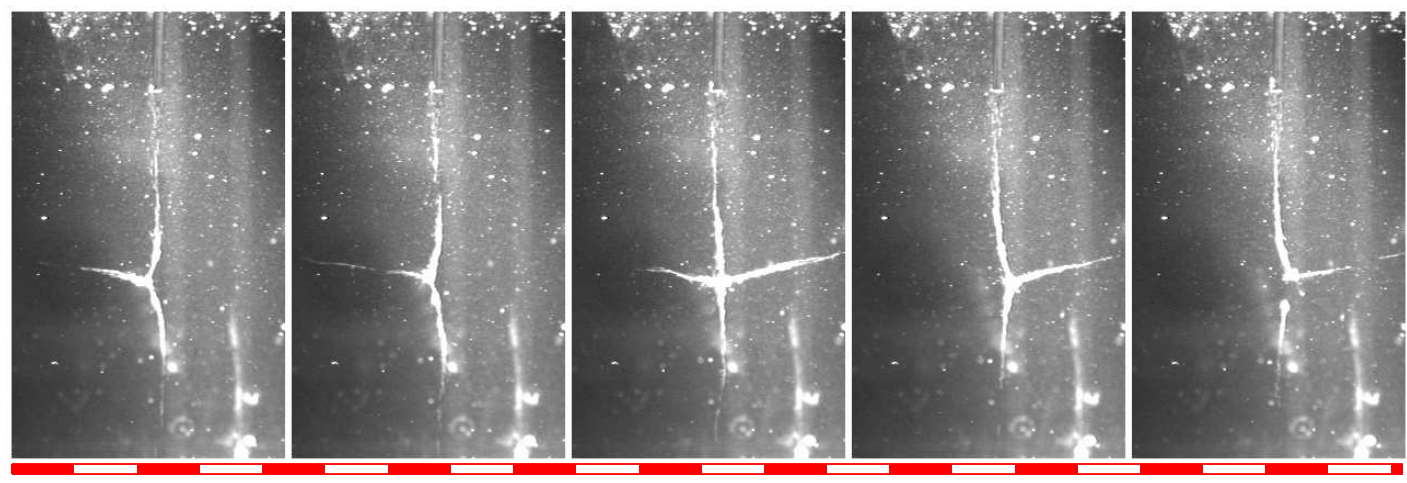

$5 \mathrm{~mm}$

Abbildung 5.31: Streamer-Kreuz mit drei bzw. vier Armen. Mit dem wechselseitigen Versiegen der seitlichen Blasenquellen zeigt sich eine seitliche Verschiebung des Schwerpunkts um ungefährt $1 \mathrm{~mm}$ bedingt durch die sekundäre Bjerkneskraft; Aufnahmen von Krefting [19]; Bildabstand $0.1 \mathrm{~s}$, Belichtungszeit $16 \mathrm{~ms}, \nu \approx 24 \mathrm{kHz}, \hat{p}_{\mathrm{A}} \approx 120 \mathrm{kPa}$.

Die Neigungen der seitlichen Blasenbahnen in Abbildung 5.31 lassen darauf schließen, dass sich die Quellpunkte dieser Streamerarme etwas oberhalb der Position des Druckbauchs befinden.

Durch zufällige Unterbrechungen einer Blasenquelle wird in der verbleibenden dreiarmigen Struktur die Wirkung der sekundären Bjerkneskraft deutlich. Die Blasen am Ende des verbleibenden seitlichen Arms lenken die von oben bzw. unten ausgebildeten Streamerarme ab, so dass sich der Schwerpunkt der Figur verlagert.

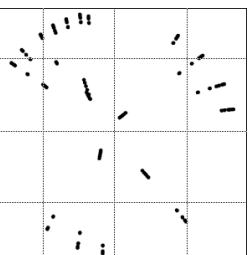


Für eine qualitative Nachbildung des Einflusses der sekundären Bjerkneskraft wird das in Abschnitt 4.5 vorgestellte Partikelmodell eingesetzt. Das Stehwellenfeld wird nach der Beschreibung in Abschnitt 3.1 analytisch angegeben, entsprechend den von Krefting [19] gemachten Angaben:

maximale Schalldruckamplitude: $\hat{p}_{\mathrm{A}}=120 \pm 10 \mathrm{kPa}$

Schallfeldfrequenz: $\quad \nu=24 \mathrm{kHz}$

Die Initialisierungspunkte der Blasen sind gemäß den Aufnahmen in Abbildung 5.31 gewählt:

\begin{tabular}{ccc} 
Blase & $\vec{x}_{\text {init }}[\mathrm{mm}]$ & $\vec{v}_{\text {init }}[\mathrm{mm} / \mathrm{s}]$ \\
\hline 1 & $(5,0,0.5)$ & $(0,0,0)$ \\
2 & $(-5,0,0.5)$ & $(0,0,0)$ \\
3 & $(0,0,5)$ & $(0,0,0)$ \\
4 & $(0,0,-5)$ & $(0,0,0)$
\end{tabular}

Alle Blasen werden mit $R_{0}=5 \mu \mathrm{m}$ initialisiert. Es werden Stickstoffblasen angenommen $\left(D_{\mathrm{R}}=2 \cdot 10^{-9} \mathrm{~m}^{2} / \mathrm{s}, c_{\mathrm{sat}}=0.6 \mathrm{~mol} / \mathrm{m}^{3}\right)$, die durch gerichtete Diffusion gemäß der Wachstumsrate (2.35) ihren Ruheradius ändern können.

Die Blasendynamik wird durch das Keller-Miksis-Modell (2.10) im rein isothermen Fall $(\eta=1)$ beschrieben.

Der Reibungskoeffizient wird der Betrachtung in Abschnitt 4.4 folgend auch hier mit $\alpha=11$ angenommen.

Neue Blasen werden unabhängig voneinander an den Quellpunkten der Simulation hinzugefügt. Nach der Initialisierung an einem Quellpunkt wird dieser Punkt für eine aus dem Intervall $[200,400] \cdot \tau_{\text {osz }}=[8.3,16.7]$ ms zufällig gewählte Zeitspanne als inaktiv angenommen.

Über den in Abbildung 5.32 „,belichteten“ Zeitraum von 146 ms wurden für die dreiarmige Streamerfigur im Ganzen 68 Blasentrajektorien berechnet, im Fall der vierarmigen Figur 89 Trajektorien.

An der Verästelung der Streamerarme zum Zentrum hin ist zu erkennen, dass der Ort der sich ausbildenden zentralen Blase bzw. Blasenwolke in einem Bereich von etwa $\pm 0.5 \mathrm{~mm}$ in $x$ - und $z$-Richtung schwankt.

Bei einer inaktiven Blasenquelle ist eine deutliche Verlagerung des Schwerpunktes der Figur zu sehen. Der in Abbildung 5.32 (links) gezeigte Fall der versiegten rechten Quelle führt zu einer Auslenkung des Schwerpunktes von etwa $0.6 \mathrm{~mm}$.

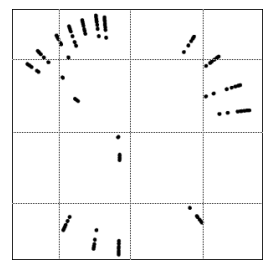



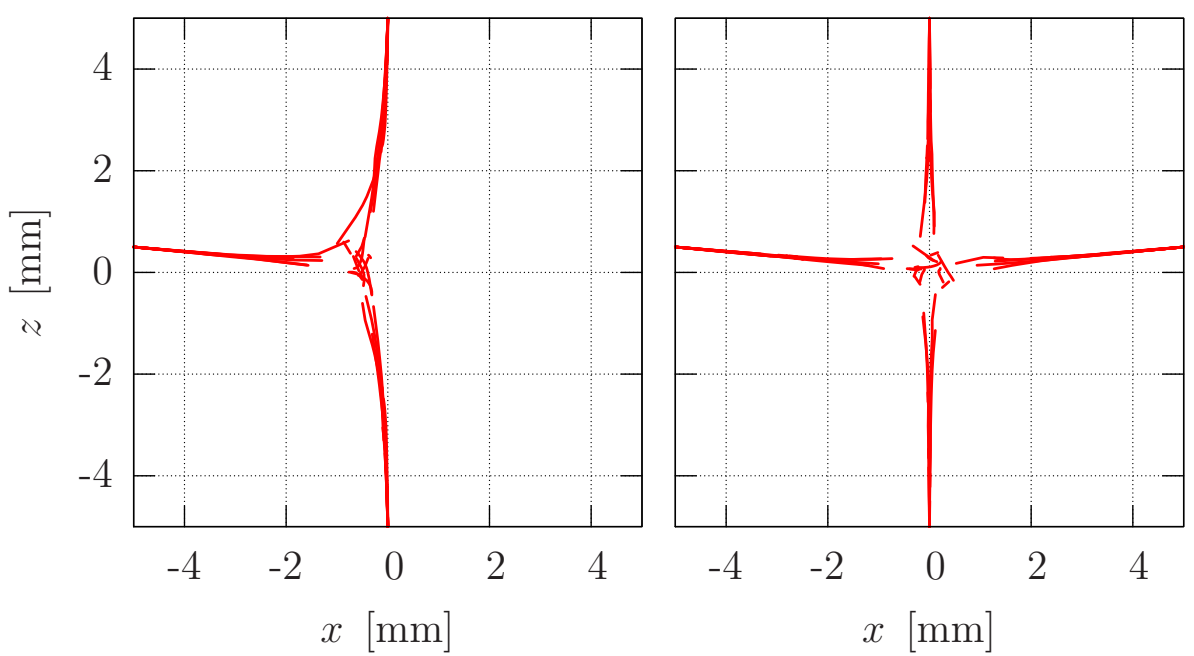

Abbildung 5.32: Ergebnis der Partikelsimulation ausgehend von vier symmetrisch am Rand positionierten Quellpunkten, abgebildet sind die Blasentrajektorien über einen Zeitraum von 146 ms. Links: Ausbildung eines dreiarmigen Streamers, bei einer inaktiven Blasenquelle; rechts: Aufbau eines vierarmigen Streamers; $\nu=24 \mathrm{kHz}, \hat{p}_{\mathrm{A}}=125 \mathrm{kPa}, R_{0}=5 \mu \mathrm{m}$, $\alpha=11, c_{\infty} / c_{\mathrm{sat}}=100 \%$.

Dieser Wert entspricht sehr gut der im Experiment beobachteten und in Abbildung $5.31 \mathrm{zu}$ erkennenden Auslenkung. Dagegen liegt der Schwerpunkt im Fall der symmetrisch aktiven Quellen in Abbildung 5.32 (rechts) im Zentrum.

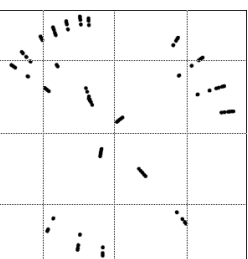




\subsection{Blasen-Schallfeld-Rückkopplung}

Im Folgenden wird die Rückwirkung der Blasenverteilung auf das Schallfeld in einem akustischen Resonator an einem Beispielexperiment untersucht.

Durch das gezielte Einbringen von Störstellen können die Punkte der Blasenentstehung beim Überschreiten der Kavitationsschwelle vorgegeben werden. So wurde von KREFTing [19] eine zweidimensionale Blasenstrukturen initialisiert, indem ein Drahtring (Durchmesser etwa $3.5 \mathrm{~cm}$ ), in die die aus Abschnitt 3.2 bekannte Hellma-Küvette eingetaucht wurde. Die sich dabei ausbildenden Strukturen zeigen ein ähnliches Verhalten zu den in der Beobachtung und Behandlung aufwändigeren dreidimensionalen Strukturen, die in Abschnitt 5.3 und 5.4 gezeigt wurden.
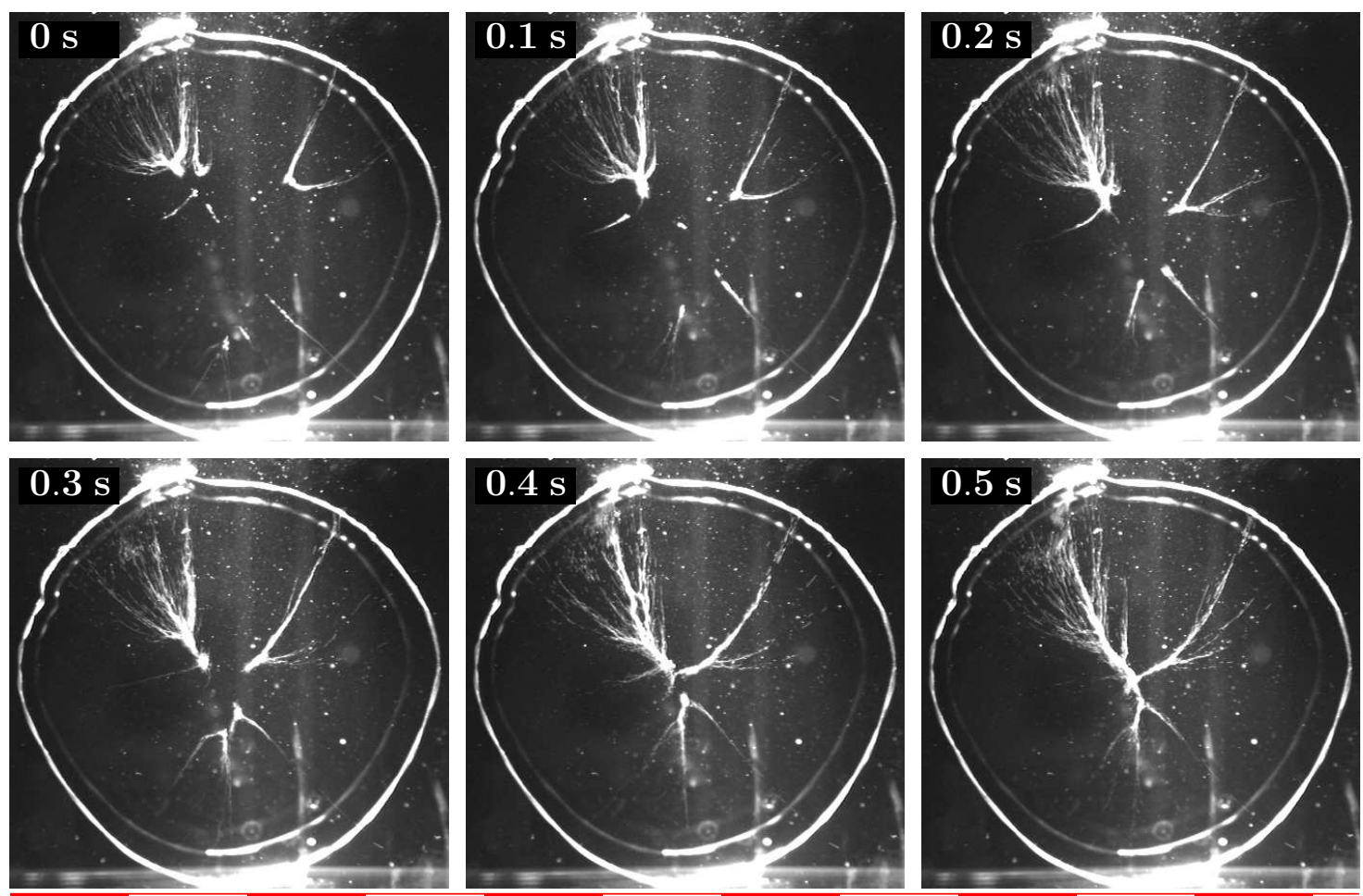

$1 \mathrm{~cm}$

Abbildung 5.33: Entwicklung einer zweidimensionalen von einem Ring ausgehenden Blasenstruktur; Aufnahme von Krefting [19]; Bildabstand $0.1 \mathrm{~s}$, Belichtungszeit $16 \mathrm{~ms}$, Auflösung $83.3 \mu \mathrm{m} /$ Pixel, Bildaufnahmerate 60 Bilder $/ \mathrm{s}, \nu=24 \mathrm{kHz}, \hat{p}_{\mathrm{A}} \approx 180 \mathrm{kPa}(t=0 \mathrm{~s}) \ldots 120 \mathrm{kPa}(t=0.5 \mathrm{~s})$.

Durch Eintauchen des Ringes in den mit Leitungswasser befüllten HellmaResonator wird eine Vielzahl von Blasenquellen eingebracht, die nach einigen

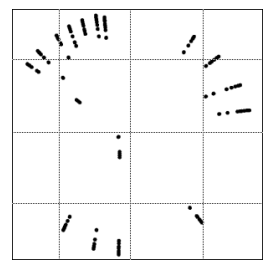


Sekunden versiegen. In dieser Zeit bildet sich aus den Blasenkeimen eine zweidimensionale Streamerfigur innerhalb des Ringes, wie sie in Abbildung $5.33 \mathrm{zu}$ sehen ist. Durch die Beleuchtung kommt es zu einer Reflexion des Ringes in der Küvettenrückwand, welche in den Aufnahmen als ein scheinbarer innerer Ring zu sehen ist.

Die langzeitbelichteten Aufnahmen in Abbildung 5.33 wurden mit der in Anhang B näher spezifizierten Pulnix-CCD-Kamera gemacht. Diese von Krefting [19] aufgenommene Sequenz zeigt eine typische Streamerfigur in dem in der $(1,1,1)$ Mode betriebenen Resonator.

Bei der Beobachtung der in Abbildung 5.33 gezeigten Figur fällt besonders die zunächst blasenleere Mitte des Ringes auf. Die von dem Drahtring ausgehenden Streamerarme vereinigen sich in mehreren Schwerpunkten der Figur in einem bestimmten Abstand um den Mittelpunkt herum. In der zeitlichen Entwicklung der Figur zeigt sich, dass sich diese Streamerarme verlängern, bis sich schließlich ein zentraler Streamerknoten ausbildet. Gleichzeitig sinkt der von KREFTING [19] berührungslos mit einem Vibrometer bestimmte Schalldruck im Druckbauch der Küvette von etwa $180 \mathrm{kPa}$ auf etwa $120 \mathrm{kPa}$ ab.

Der Erklärungsansatz dieser Beobachtung geht davon aus, dass die Blasenkonzentration zu einer Beeinflussung des Schallfeldes durch die in Abschnitt 3.3.4 behandelte Resonanzverschiebung des Systems führt, was im Folgenden näher betrachtet wird.

\subsubsection{Numerische Analyse}

In diesem Abschnitt wird die Resonanzfrequenz der Küvette für verschiedene im Experiment beobachtete räumliche Blasendichteverteilungen bestimmt.

In der numerischen Betrachtung wird wieder die modifizierte HelmholtzGleichung (3.21) eingesetzt. Hierbei ist der Ring nur als unendlich dünne Blasenquelle angenommen, wodurch sich die aus Abbildung 3.5 bekannte numerische Resonanzfrequenz der blasenfreien Hellma-Küvette der Grundmode von $\nu_{\text {res }}=23.647 \mathrm{kHz}$ einstellt. Im Experiment verschiebt sich die Grundmode durch den eingebrachten Drahtring etwas nach oben (auf $24 \mathrm{kHz}$ ), was aber in der Rechnung vernachlässigt wird. Dies ist gerechtfertigt, da es sich um eine über die gesamte Aufnahme konstante Beeinflussung handelt und somit um eine gleichbleibende Resonanzverschiebung.

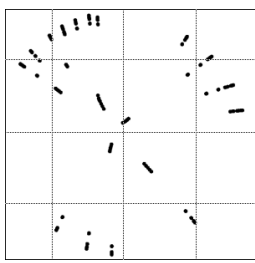




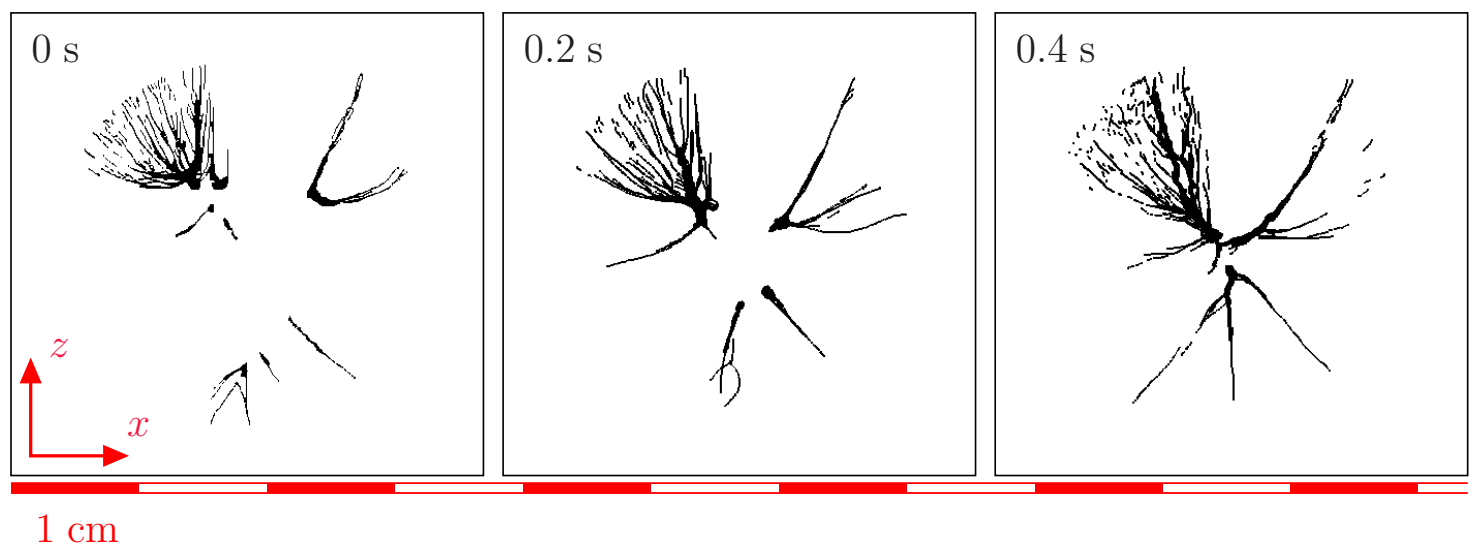

Abbildung 5.34: Darstellung der aus den in Abbildung 5.33 gezeigten Strukturen extrahierten Blasenpositionen, die als Vorgabe für die Rückkopplung auf das Schallfeld eingesetzt werden.

Aus den langzeitbelichteten Aufnahmen wurden die in Abbildung 5.34 gezeigten Blasenbahnen mit einer von Hand nachbearbeiteten Schwellwertfilterung extrahiert und als Grundlage für die Angabe der Blasenanzahldichte $n(\vec{x})$ auf einem $50 \times 50$ Gitter verwendet. Durch diese Rasterung erfolgt eine Diskretisierung der Blasenzahldichte $n_{i}$. Die Gitterabmessungen werden jeweils der $x$ - und $z$ Ausdehnung der Blasenverteilung angepasst und die Zelleinteilung in äquidistanten Abständen je Achse vorgenommen. Jeder auftretende Blasenpunkt wird so einer Zelle zugeordnet. Aus der Gesamtzahl der Blasen je Zelle $N_{i}$ ergibt sich für einheitliche Blasen mit einem Ruheradius von $R_{\mathrm{n}}=5 \mu \mathrm{m}$ das Gesamtblasenvolumen in der Zelle:

$$
V_{i}=N_{i} \cdot \frac{4}{3} \pi R_{\mathrm{n}}^{3}, \quad i: 50 \times 50 \text { Zellen }
$$

Die Ausdehnung der quasi zweidimensionalen Blasenverteilung entlang der Tiefenachse ( $y$-Achse) wird gemäß einer typischen Streamerbreite von $0.5 \mathrm{~mm}$ geschätzt. Für die Ausweitung der Figur auf das Dreidimensionale werden daher die in der $x$-z-Ebene liegende Zellen entlang der $y$-Achse gaußförmig mit der Halbwertsbreite von $\Delta_{\text {hwb }}=500 \mu \mathrm{m}=100 \cdot R_{\mathrm{n}}$ verschmiert. Ausgeführt wird dieser Verlauf der Blasenzahl in $y$-Richtung über $2 \mathrm{~mm}$ symmetrisch um $y=0$ mit einer Einteilung in 20 Zellen entlang der $y$-Achse. Außerhalb dieses Bereichs $(|y|>2 \mathrm{~mm})$ wird die Blasenzahl auf Null gesetzt. Während hier eine Zelle sowohl in $x$-als auch in $z$-Richtung etwa $0.6 \mathrm{~mm}$ lang ist, ist sie in $y$-Richtung $0.1 \mathrm{~mm}$ lang.

Für die Skalierung des Blasenfeldes wird von einer Gesamtblasenzahl von $N=$

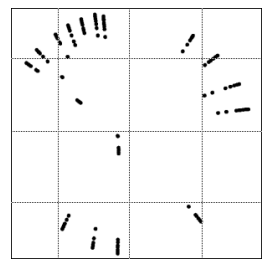


$10^{4}$ Blasen ausgegangen:

$$
\varepsilon \cdot \sum_{i} N_{i} \stackrel{!}{=} 10^{4} \text { Blasen, } \quad i: 50 \times 20 \times 50 \text { Zellen }
$$

Durch den Skalierungsfaktor $\varepsilon$ werden die ausgezählten Blasen je Zelle entsprechend der geforderten Gesamtblasenzahl normiert. Die diskrete Verteilungsfunktion der Blasenzahldichte $n_{i}$ bezieht diese normierte Blasenzahl auf das einheitliche Zellvolumen $V_{\mathrm{Z}}$ :

$$
n_{i}=\frac{\varepsilon \cdot N_{i}}{V_{\mathrm{Z}}}
$$

Diese Blasenzahldichte geht ein in die in Abbildung 5.35 aufgetragene modifizierte Schallgeschwindigkeit (3.22) in dem Blasen-Flüssigkeitsgemisch, die wiederum zur Lösung der modifizierten gedämpften Helmholtz-Gleichung (3.25) eingesetzt wird. Aus deren Lösungen zu unterschiedlichen Frequenzen ergibt sich die Resonanzkurve des Systems.

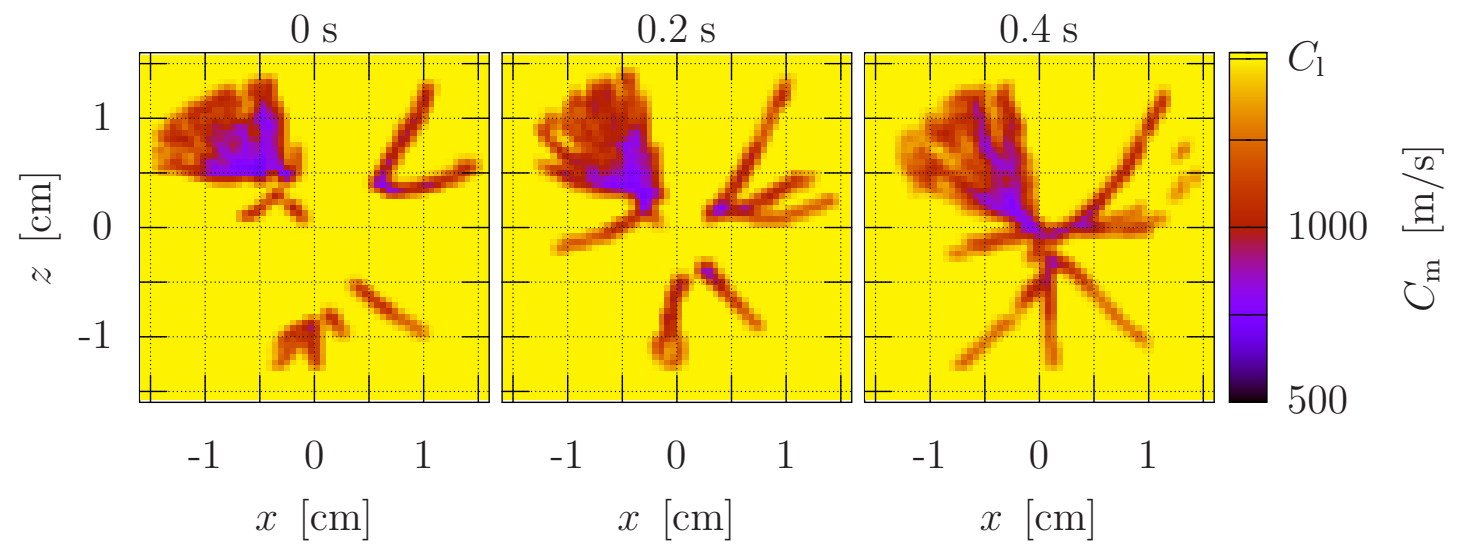
Abbildung 5.35: Auftragung der modifizierten Schallgeschwindigkeit (3.22) im Blasen-Flüssigkeitsgemisch, ausgehend von den in Abbildung 5.34 gezeigten auf ein $50 \times 50$ Gitter übertragenen experimentell erfassten Blasenverteilungen; $\nu=23.647 \mathrm{kHz}, R_{\mathrm{n}}=5 \mu \mathrm{m}$, Schallgeschwindigkeit ohne Blasen $C_{1}=1482 \mathrm{~m} / \mathrm{s}$.

Abbildung 5.36 zeigt zunächst die Resonanzkurve des blasenfreien Systems, welche dem in Abschnitt 3.2 betrachteten idealen Hellma-Resonator entspricht. Aus dieser in Abbildung 3.5 gezeigten Betrachtung ergibt sich der Dämpfungsfaktor von $D=3.26 \cdot 10^{5} \mathrm{~kg} / \mathrm{m}^{3} / \mathrm{s}$.

Mit dem Auftreten der Blasenstrukturen verschiebt sich die Resonanzkurve zu kleineren Frequenzen, wie in der Auftragung in Abbildung 5.36 für $t=0 \mathrm{~s}$ und

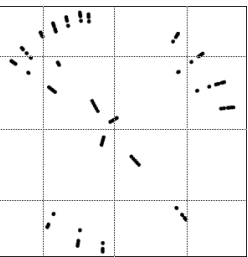


$t=0.4 \mathrm{~s}$ gezeigt. Entsprechend sinkt die Schalldruckamplitude für das bei konstanter Frequenz $\nu=23.647 \mathrm{kHz}$ betriebene System entlang der Flanke der Resonanzkurve ab. Durch die Veränderung der Blasenfigur, wie sie in Abbildung 5.34 über die Zeit zu verfolgen ist, verlagert sich die Resonanzkurve weiter, so dass die Schalldruckamplitude noch stärker absinkt. Dies geschieht bei einer konstant gehaltenen Blasenanzahl von $10^{4}$ Blasen allein durch die veränderte räumliche Verteilung der Blasen.

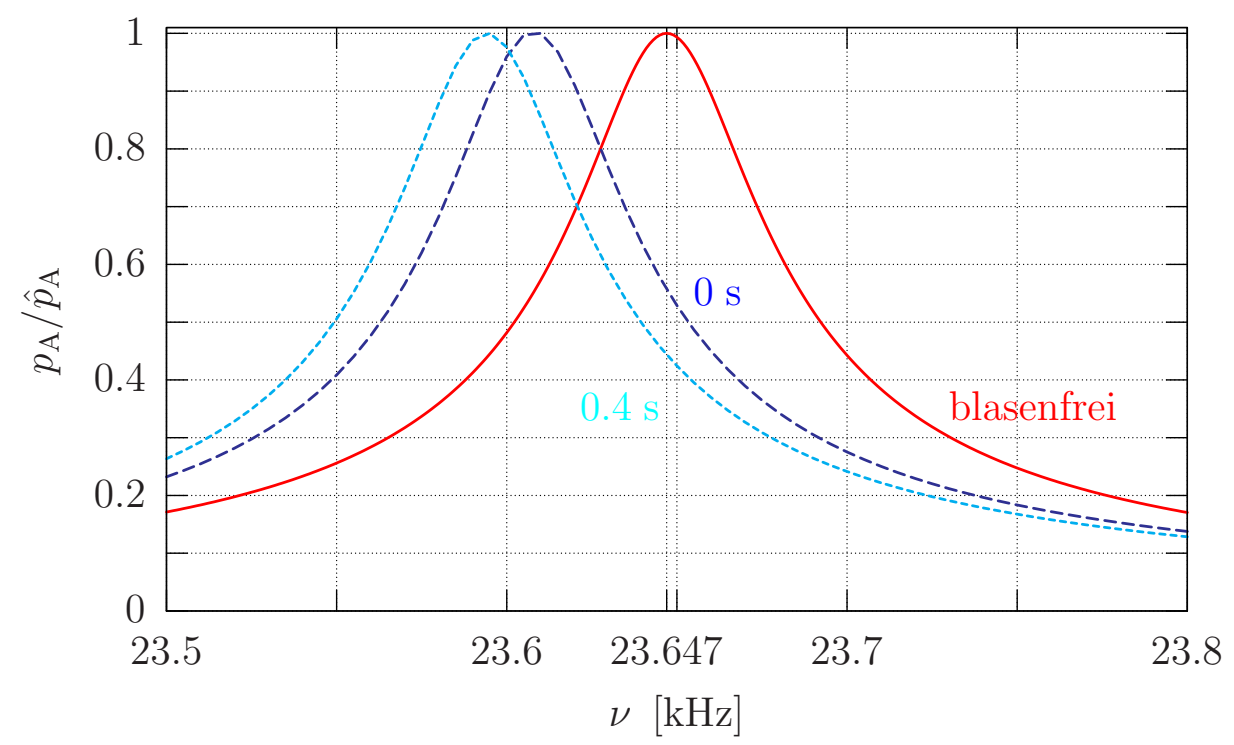

Abbildung 5.36: Verschiebung der Resonanz in Abhängigkeit der in Abbildung 5.34 gezeigten Blasenverteilungen, berechnet als Lösungen der gedämpften modifizierten Helmholtz-Gleichung (3.25). Gegenüber dem blasenfreien System ist die Resonanzkurve für den blasenhaltigen Resonator mit den Blasenverteilungen zu den Zeitpunkten $t=0 \mathrm{~s}$ und $t=0.4 \mathrm{~s}$ aufgetragen; $N=10^{4}$ Blasen, $D=3.26 \cdot 10^{5} \mathrm{~kg} / \mathrm{m}^{3} / \mathrm{s}$.

Abbildung 5.37 zeigt nun diese Verschiebung der Lage der $(1,1,1)$-Resonanz sowie das Absinken der Schalldruckamplitude bei $\nu=23.647 \mathrm{kHz}$ in Abhängigkeit von der Gesamtblasenzahl, mit der die aus Abbildung 5.34 bekannten Verteilungen zu $t=0 \mathrm{~s}$ und $t=0.4 \mathrm{~s}$ skaliert werden.

Das System wird weiterhin mit der Resonanzfrequenz des blasenfreien Systems getrieben $(\nu=23.647 \mathrm{kHz})$. Mit der angenommenen Gesamtblasenzahl von $N=10^{4}$ Blasen lässt sich aus Abbildung 5.37 für den Zeitpunkt $t=0$ s ein Absinken der Amplitude auf etwa $55 \%$ der maximalen Amplitude des blasenfreien Systems ablesen. Mit der zeitlichen Entwicklung der Blasenstruktur fällt die Amplitude entsprechend der Resonanzverschiebung weiter ab, bei $t=0.4 \mathrm{~s}$

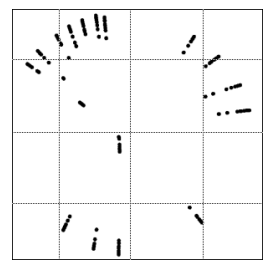




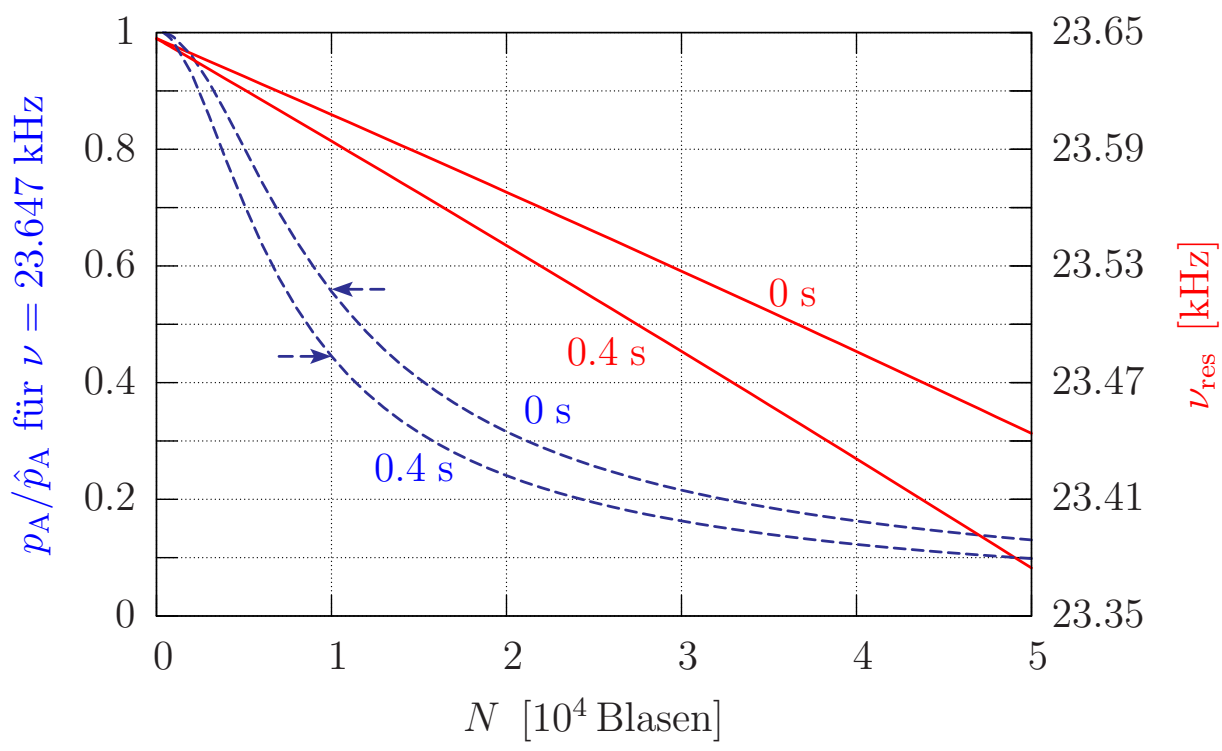

Abbildung 5.37: Darstellung der Verschiebung der Resonanzfrequenz (rot) mit dem dabei auftretenden Absinken der Schalldruckamplitude (blau) bei $\nu=23.647 \mathrm{kHz}$ in Abhängigkeit von der Gesamtblasenzahl $N$. Die Zeiten $0 \mathrm{~s}$ und $0.4 \mathrm{~s}$ indizieren die entsprechenden räumlichen Blasenbzw. Schallgeschwindigkeitsverteilungen aus Abbildung 5.34 bzw. 5.35. Die Pfeile zeigen die Amplituden zu den beiden Zeiten für $N=10^{4}$ Blasen an.

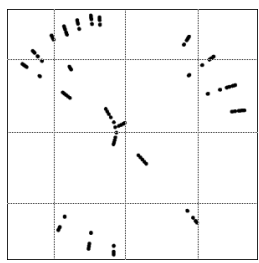


liegt sie bei etwa $45 \%$. Nach der von Krefting [19] für die in Abbildung 5.33 gezeigten Aufnahmen angegebenen Schalldruckamplitude von $\hat{p}_{\mathrm{A}} \approx 180 \mathrm{kPa}$ für $t=0 \mathrm{~s}$ ergibt sich für $t=0.4 \mathrm{~s}$ eine Amplitude in realistischer Größenordnung: $\hat{p}_{\mathrm{A}}=180 \mathrm{kPa} \cdot 45 / 55=147 \mathrm{kPa}$. Jedoch erscheint die sich entsprechend ergebende Schalldruckamplitude des blasenfreien Systems deutlich überhöht: $\hat{p}_{\mathrm{A}}=180 \mathrm{kPa} \cdot 100 / 55=327 \mathrm{kPa}$. Dieser Wert konnte im Experiment nicht realisiert werden, da vorher immer Kavitation einsetzt (die Küvette also nicht blasenfrei bleibt). Einen starken Einfluss auf den Amplitudenabfall hat natürlich die angenommene Halbwertsbreite (über die Dämpfungskonstante $D$ ). Je schmaler die Resonanz ausfällt, umso größer die Absenkung bei Resonanzverschiebung. Der benutzte Wert stammt aus der blasenfreien Betrachtung und wurde nicht weiter modifiziert.

\subsubsection{Partikelmodellierung}

Im folgenden Schritt werden numerisch aus dem Partikelmodell gewonnene Blasenbahnen in die Berechnung des sich ausbildenden Schallfeldes rückgekoppelt.

Die Tabellierungen der Kraftkoeffizienten (4.16a) - (4.16e) erfolgt für die experimentell von KRefting [19] eingestellte Frequenz von $\nu=24 \mathrm{kHz}$. Da in der Modellierung der wasserbefüllten Küvette der eingebrachte Drahtring nicht berücksichtigt wird, liegt die numerische Resonanzfrequenz der (1, 1, 1)-Mode etwas niedriger, $\nu_{\text {res }}=23.647 \mathrm{kHz}$, was aber keinen weiteren Einfluss nimmt.

Zunächst wird für die in Resonanz $(\nu=23.647 \mathrm{kHz})$ getriebene blasenfreie Hellma-Küvette die Schalldruckverteilung in der (1, 1, 1)-Mode als Lösung der Helmholtz-Gleichung (3.3) bestimmt. Nach den in Kapitel 3 vorangegangenen Untersuchungen dieses Systems kann davon ausgegangen werden, dass bei der hier anzunehmenden recht geringen Gesamtblasenzahl $(N \approx 5000$ Blasen) keine signifikante Änderung der Schallfeldgeometrie auftreten wird. Lediglich eine Verschiebung der Resonanz ist zu erwarten, ähnlich der bereits in Abbildung 5.36 gezeigten Verschiebung. Um das Absinken der Schalldruckamplitude über die Verschiebung der Resonanzkurve bestimmen zu können, wird mit der auch zuvor benutzten Dämpfung von $D=3.26 \cdot 10^{5} \mathrm{~kg} / \mathrm{m}^{3} / \mathrm{s}$ gerechnet, die für die wasserbefüllte Hellma-Küvette in Abschnitt 3.2 bestimmt wurde.

Die Wahl der Startpositionen der Blasen ist motiviert durch die in Abbildung 5.33 gezeigten Aufnahmen der Blasenstruktur. Entsprechend werden die Quellpunkte auf einem Ring um den Druckbauch angeordnet. Von jedem dieser Punkte

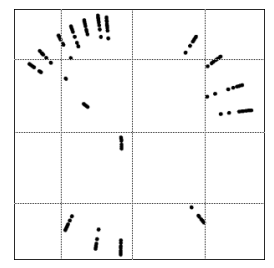


wird alle 100 bis 200 Schallfeldperioden zufällig eine neue Blase in die Simulation gesetzt.

Die maximale Schalldruckamplitude wird von KREFTING [19] mit $\hat{p}_{\mathrm{A}} \approx 180 \mathrm{kPa}$ angegeben. Da diese Angabe sich auf ein System bezieht, in dem bereits eine Blasenstruktur vorhanden ist, ist zu vermuten, dass bereits eine Verschiebung der Resonanz relativ zum blasenfreien System vorliegt.

Der erste Lauf des Partikelmodells (4.17) startet mit den Anfangsbedingungen eines blasenfreien Systems $\nu_{\text {res }}=23.647 \mathrm{kHz}$ und $\hat{p}_{\mathrm{A}}=185 \mathrm{kPa}$. Die sich bis zur Zeit $t=0.11 \mathrm{~s}$ ausgebildete Struktur ist in Abbildung 5.38 (oben) gezeigt.

Die diskretisierte Funktion der Blasenzahldichte $n_{i}$ ergibt sich durch ein Auszählen der Blasen in einem äquidistanten Raster, das über den von der Blasenstruktur ausgefüllten Bereich in der $x$-z-Ebene gelegt wird, hier ein $30 \times 30$ Gitter. Die Anzahl der Blasen pro Zelle des Rasters wird auf das einheitliche Zellenvolumen $V_{\mathrm{Z}}$ bezogen:

$$
n_{i}=\frac{\varepsilon \cdot N_{i}}{V_{\mathrm{Z}}}, \quad \varepsilon \cdot \sum_{i} N_{i} \stackrel{!}{=} 5000, \quad i: 30 \times 10 \times 30 \text { Zellen }
$$

Vor der Normierung auf die in Abschnitt 5.7.1 abgeschätzte Gesamtblasenzahl $(N=5000)$ wird die Blasenverteilung in die dritte Raumdimension auf ein $30 \times$ $10 \times 30$ Gitter erweitert. Wie bereits in Abschnitt 5.7.1 geschieht dies auch hier durch eine gaußförmige Verschmierung der Blasenanzahl entlang der $y$-Achse, hier über einen Bereich von $200 \mu \mathrm{m}$. Die Halbwertsbreite ergibt sich aus einer groben Abschätzung der zu erwartenden maximalen Blasenradien $\Delta_{\text {hwb }}=75 \mu \mathrm{m}=$ $15 \cdot R_{\mathrm{n}}$.

Die räumliche Verteilung der Blasenzahldichte wird alle 2500 Schallfeldperioden (entsprechend $t=0.11,0.21,0.32,0.42 \mathrm{~s}$ ) aus den Trajektorien des letzten Zeitschritts neu bestimmt.

Aus der oben beschriebenen Methode der Zuweisung der Blasenzahldichte aus „langzeitbelichteten“ Trajektorien ist zu erkennen, dass das Partikelmodell nicht wirklich jede einzelne Blase modelliert. Es handelt sich eher um etwa 100 typische Blasen, deren Verteilung über kurze Zeitspannen (2500 Schallfeldperioden) gemittelt und anschließend auf eine Blasengesamtzahl skaliert werden.

Neben den „langzeitbelichteten" Blasenbahnen im Zeitraum von 0 bis 0.11 s zeigt Abbildung 5.38 (oben) die Verteilung der Schallgeschwindigkeit (3.23), ebenfalls in der $x$-z-Ebene $(y=0)$.

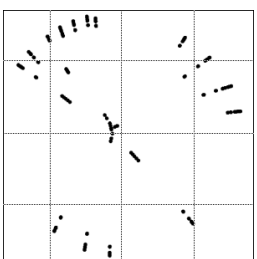




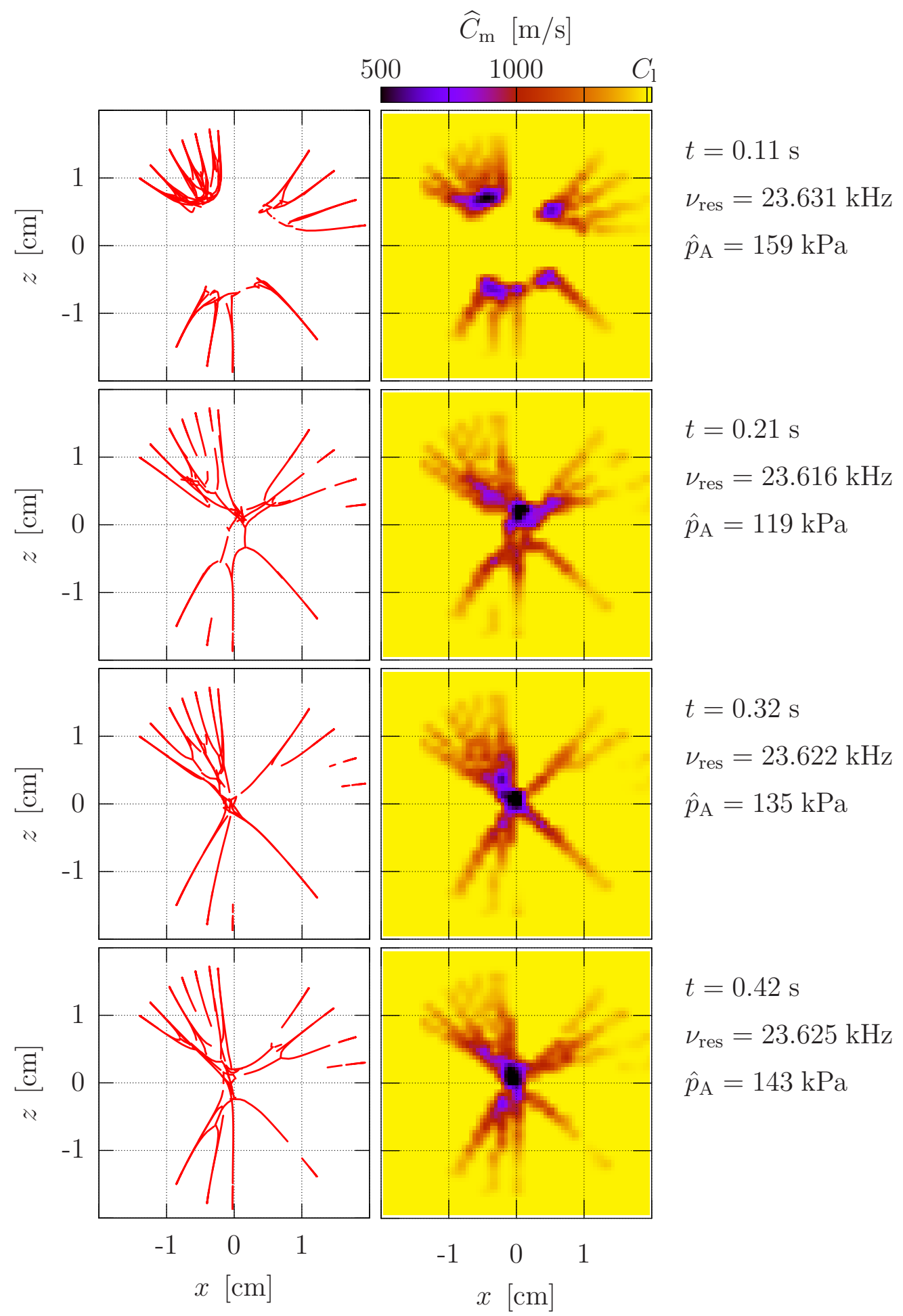

Abbildung 5.38: Die Partikelmodellierung wird alle 2500 Schallfeldperioden $(\sim 0.11 \mathrm{~s})$ unterbrochen, um die veränderte Resonanz des Systems zu ermitteln. Über den betrachteten Zeitraum ändert sich die qualitative Form der Blasenstruktur bei konstanter Gesamtblasenzahl bedingt durch die Blasenverteilung. Links sind die Blasentrajektorien abgebildet, rechts die Verteilung der Schallgeschwindigkeit (3.23); $N=5000$ Blasen, $\nu=23.647 \mathrm{kHz}$.

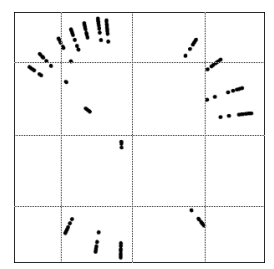


Die ausgedehnte Blasenverteilung führt zunächst zu einem starken Absinken der Schalldruckamplitude. Mit der daraus resultierenden Vorzeichenumkehr der primären Bjerkneskraft werden die Blasen in das bis dahin entvölkerte Zentrum gezogen, wobei sich die Figur schließt $(t=0.21 \mathrm{~s})$. Mit der Konzentration der Blasen um das Zentrum stellt sich die Resonanzfrequenz auf einen Wert um $23.62 \mathrm{kHz}$ ein. In der Auftragung der Schallgeschwindigkeitsverteilungen in Abbildung 5.38, die auf der Blasenverteilung beruht, sind die jeweiligen Schwerpunkte der Figuren, das heißt die Regionen niedriger Schallgeschwindigkeit, gut zu sehen. Für die relativ stabile Figur bei $t=0.32 \ldots 0.42 \mathrm{~s}$ ist zu erkennen, dass das Zentrum der Figur leicht um den geometrischen Mittelpunkt wandert.

Die Eigenfrequenzanalyse liefert die verschobene Resonanzfrequenz des Systems entsprechend der in die modifizierte Helmholtz-Gleichung (3.21) eingegangene Blasenzahldichte $n(\vec{x})$, die sich in den in Abbildungen 5.38 gezeigten Schallgeschwindigkeitsverteilungen ausdrückt. Die Blasenzahldichte wurde auf eine Gesamtanzahl von $N=5000$ Blasen skaliert. Über die Verschiebung der Resonanzkurve des Systems wird das Absinken der Schalldruckamplitude bestimmt und die Schallfeldmode entsprechend reskaliert. Die verschobenen Resonanzkurven und die sich bei $\nu_{\text {res }}=23.647 \mathrm{kHz}$ ergebenden Amplituden sind in Abbildung 5.39 dargestellt.

Iterativ wird die Partikelmodellierung erneut gestartet. Die Blasenquellen auf dem Ring sowie die aktuellen Blasenpositionen werden beibehalten.

Eine Langzeitbetrachtung der Entwicklung von Resonanzfrequenz sowie der Schalldruckamplitude in dieser Strukturbildung zeigt Abbildung 5.40. Nach dem anfänglichen Abfallen der Resonanzfrequenz, der Verschiebung der Resonanzkurve und dem damit einhergehenden Absinken der Schalldruckamplitude, beginnt das System um die Parameter $\nu_{\text {res }} \approx 23.625 \mathrm{kHz}$ sowie $\hat{p}_{\mathrm{A}} \approx 140 \mathrm{kPa}$ zu oszillieren. Ähnlich wie auch im Experiment zu beobachten, treten also auch hier niederfrequente Fluktuationen der Struktur auf. Die in Abbildung 5.38 gezeigten Zeitschritte entsprechen den ersten vier Iterationen.

In Abbildung 5.41 sind exemplarisch drei für den gesamten in Abbildung 5.40 gezeigten Systemverlauf charakteristische Blasenstrukturen herausgegriffen $(t=$ $0.95,2.85$ und $4.44 \mathrm{~s}$ ).

$\mathrm{Zu}$ einer Schalldruckamplitude von $\hat{p}_{\mathrm{A}} \approx 160 \mathrm{kPa}$ bei $t=2.85 \mathrm{~s}$ zeigt Abbildung 5.41 (Mitte) die ausgebildete filamentartige Struktur. Die Skalierung der verwendeten Punktgröße mit der Blasengröße verdeutlicht die Ausbildung eines Schwerpunktes der Figur im Zentrum.

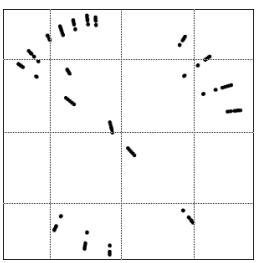




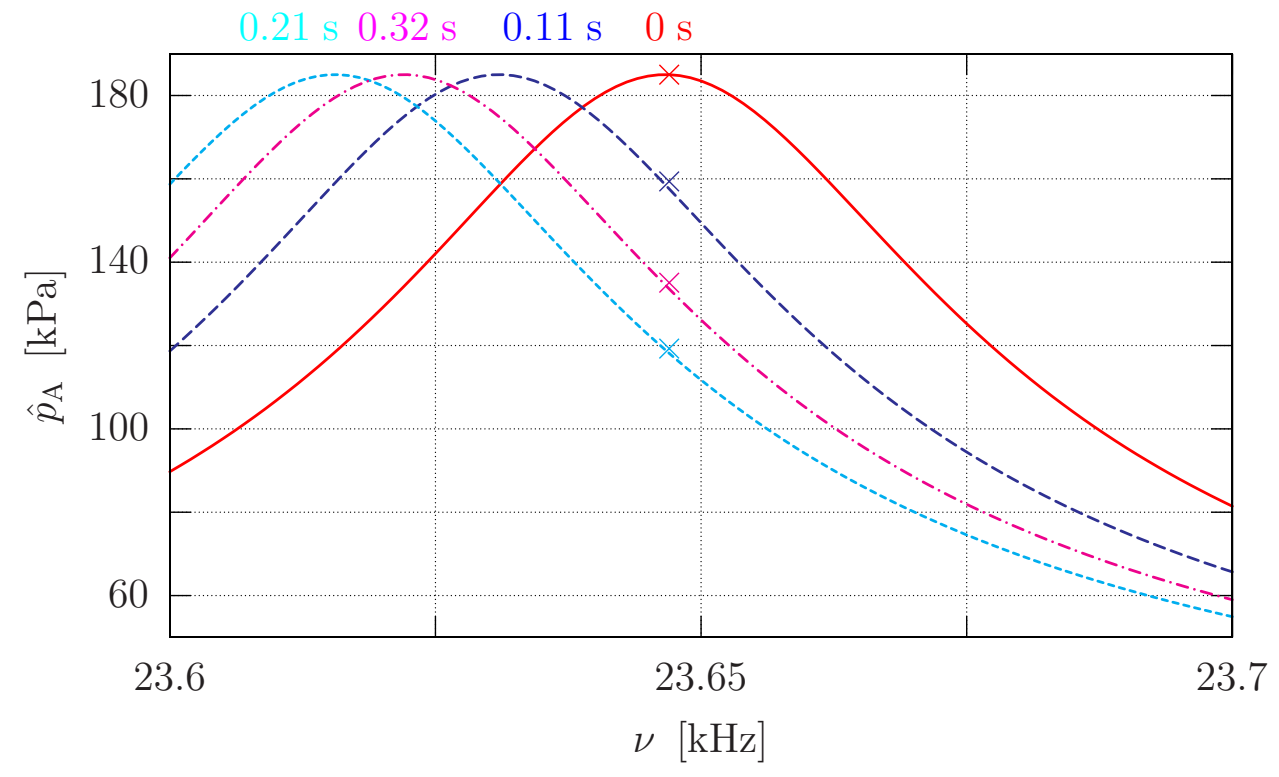

Abbildung 5.39: Die Resonanzkurve des blasenfreien Systems (0 s, rot) ergibt sich aus der gedämpften Helmholtz-Gleichung (3.5). Die Resonanzfrequenz des blasenfreien Systems liegt bei $\nu_{\text {res }}=23.647 \mathrm{kHz}$. Die Kreuze zeigen das Absinken der Schalldruckamplitude bei dieser Frequenz entsprechend den Resonanzverschiebungen aus Abbildung 5.38; $D=3.26 \cdot 10^{5} \mathrm{~kg} / \mathrm{m}^{3} / \mathrm{s}$.

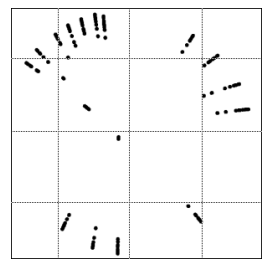




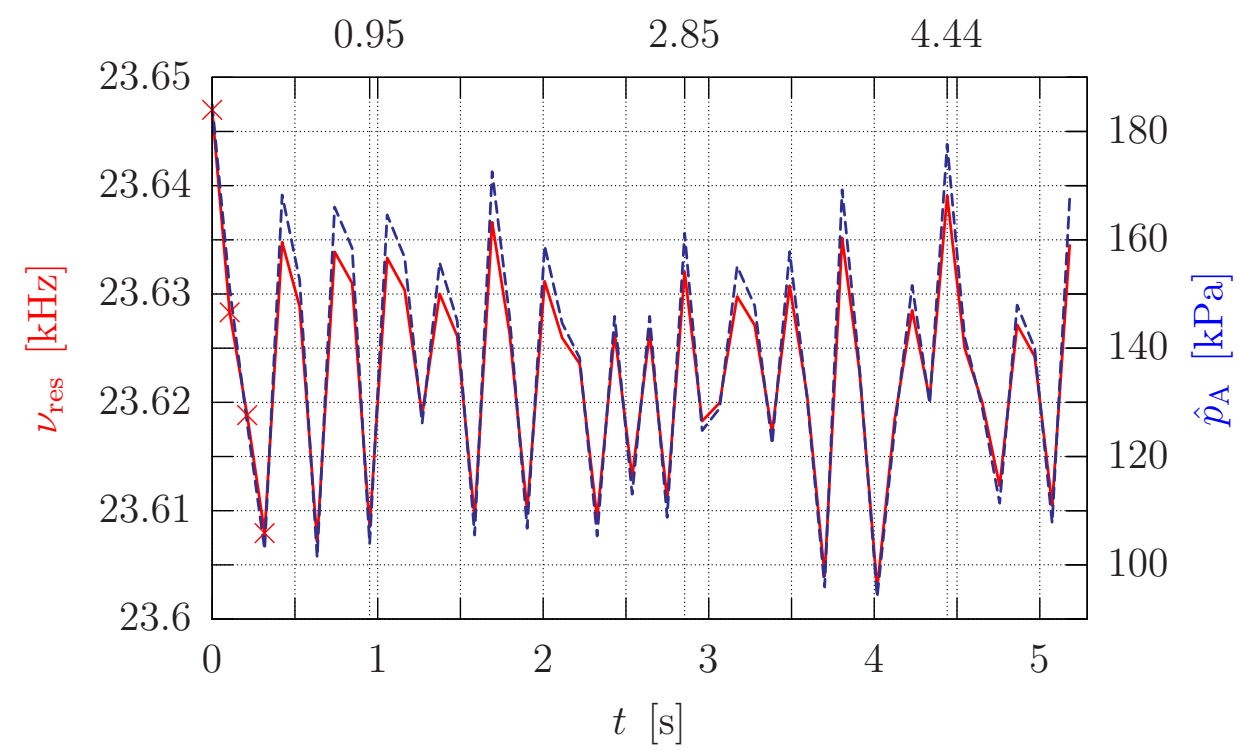

Abbildung 5.40: Betrachtung der Langzeitentwicklung der Blasenstruktur mit Resonanzverschiebung und dem daraus resultierenden Absinken und anschließenden Oszillieren der Schalldruckamplitude. Die Kreuze kennzeichnen die zuvor in Abbildung 5.38 betrachteten Zeitpunkte; $\nu_{\text {res }}(t=0)=23.647 \mathrm{kHz}, \hat{p}_{\mathrm{A}}(t=0)=185 \mathrm{kPa}$.

Kommt es im Verlauf der Dynamik zu einem Anwachsen der Schalldruckamplitude auf $\hat{p}_{\mathrm{A}} \approx 175 \mathrm{kPa}$, wie in Abbildung 5.41 (rechts) zur Zeit $t=4.44 \mathrm{~s}$ gezeigt, so wirkt das Zentrum abstoßend auf die Blasen und die Struktur reißt auseinander. Dieser Effekt entspricht dem Ausgangszustand in Abbildung 5.33, wobei das geometrische Zentrum entvölkert ist und sich mehrere Schwerpunkte ausbilden.

In der Phase einer stark abgesunkenen Schalldruckamplitude wie bei $t=0.95 \mathrm{~s}$ auf $\hat{p}_{\mathrm{A}} \approx 100 \mathrm{kPa}$ kommt es zu einem "Aussetzen“ der Strukturbildung: Die Schalldruckamplitude ist nicht mehr hoch genug, um die Blasen durch die primäre Bjerkneskraft entlang des Druckgradienten in das Zentrum der Schallfeldmode zu beschleunigen. Stattdessen bilden sich schon dicht am Rand kleine Blasenhaufen durch gegenseitige Anziehung der Blasen (sekundäre Bjerkneskraft). Ein ähnlicher Effekt wurde auch im Experiment dokumentiert. Wie es Abbildung 5.42 zeigt, kommt es durch die Bildung einer sehr großen Blase (oder eines Blasenclusters) zu einem kurzzeitigen Zusammenbrechen des Schallfeldes. Während dieser Zeitspanne verharren die Blasen nahezu unbewegt.

Wenig später bildet sich mit der Zerstörung der großen Blase erneut die filamentartige Struktur aus. Die Resonanzfrequenz steigt wieder an und damit die Schalldruckamplitude.

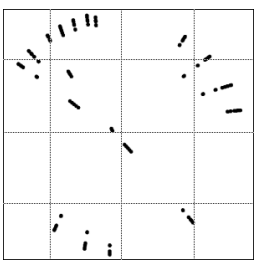




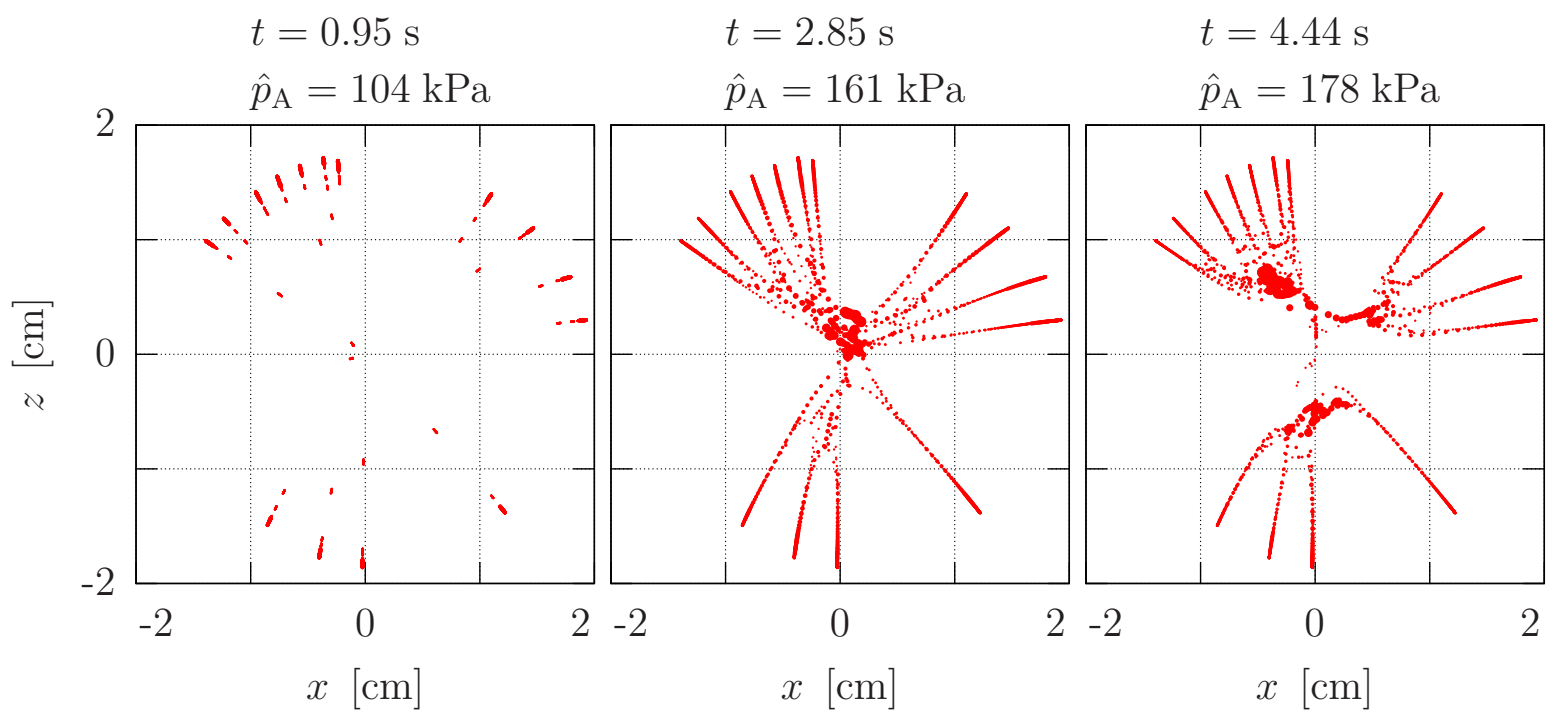

Abbildung 5.41: Exemplarische Darstellung der Blasenverteilung zu unterschiedlichen Zeitpunkten, entsprechend den in Abbildung 5.40 gekennzeichneten Punkten. Gezeigt sind die Trajektorien über eine Zeitspanne von 106 ms. Die Punktdicke skaliert mit den Ruheradien. Die Anzahl der Blasen in der Rechnung schwankt je nach neu entstehenden und kollidierten Blasen zwischen etwa 60 und 120; $R_{\mathrm{n}}=4 \ldots 20 \mu \mathrm{m}, \nu=23.647 \mathrm{kHz}$.

Die beiden Filme des Daumenkinos zeigen die zeitliche Entwicklung um die in Abbildung 5.41 für $t=2.85 \mathrm{~s}$ und $t=4.44 \mathrm{~s}$ dargestellten Blasenanordnungen. Die Gitterweite des Rasters beträgt $1 \mathrm{~cm}$. Eine Abbildung zeigt die Blasenbewegung innerhalb von $8.4 \mathrm{~ms}$, die Filme laufen über jeweils 98 Seiten, was einer Filmlänge von $0.41 \mathrm{~s}$ entspricht. Der Film über die ungeraden Seiten startet auf Seite 1 und zeigt den Ausschnitt der Strukturentwicklung von $t=2.77 \mathrm{~s}$ bis $t=3.18 \mathrm{~s}$. Auf Seite 2 beginnt der Film über die geraden Seiten, der den Filmabschnitt von $t=4.24 \mathrm{~s}$ bis $t=4.65 \mathrm{~s}$ wiedergibt.

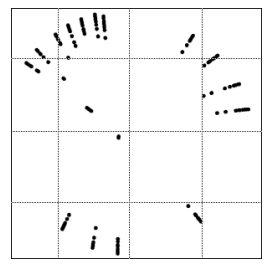



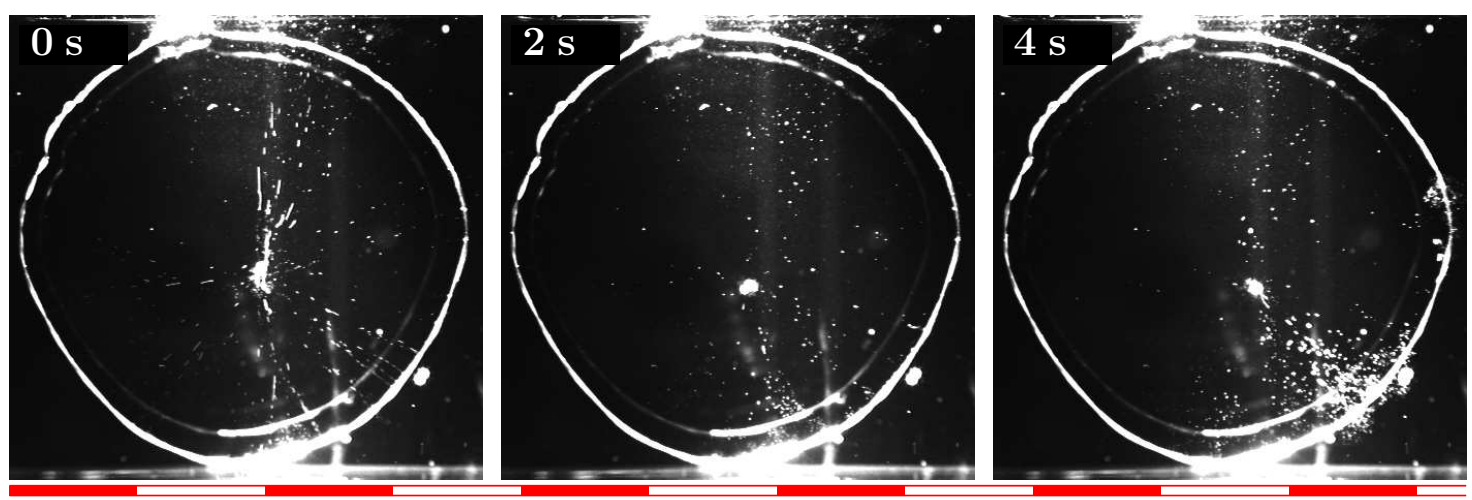

$1 \mathrm{~cm}$

Abbildung 5.42: Übergang einer filamentartigen Blasenstruktur zu einem Blasenhaufen $(t=0 \mathrm{~s})$. Während der Lebensdauer der großen Blase bricht das Schallfeld ein; die Filamentstruktur kommt zum Erliegen $(t=2 \mathrm{~s})$. Erst nach der Zerstörung der großen Blase, hier an dem Drahtring ( $t=4 \mathrm{~s}$ ), baut sich das Schallfeld wieder auf, und die Filamentstruktur bildet sich erneut aus. Aufnahme von Krefting [19]; Bildabstand $2 \mathrm{~s}$, Belichtungszeit $16 \mathrm{~ms}$.

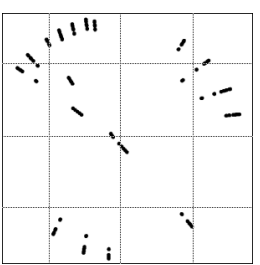




\section{Kapitel 6}

\section{Zusammenfassung und Ausblick}

In der gezeigten Arbeit wurde eine komplexe Modellierung aufgebaut, durch deren Einsatz die Simulation verschiedener Kavitationsblasenstrukturen gelang. Die Rückkopplung der sich jeweils einstellenden Struktur auf die Schallfeldausbildung wurde ebenfalls implementiert und für die FEM-Lösungen der Schallfeldmode berücksichtigt.

Der relevante Parameterraum für die auftretende radiale Blasendynamik wurde in Kapitel 2 betrachtet. Die Schallfrequenz wurde dabei auf den niedrigen Ultraschallbereich $(20 \ldots 40 \mathrm{kHz})$ festgelegt. Mit der Analyse der Oberflächenstabilität in Abschnitt 2.4 konnte die Parametrische Instabilität als wesentliches Stabilitätskriterium bestimmt werden. Das Anwachsen der Blasen durch gerichtete Diffusion wurde in Abschnitt 2.5 berechnet. Durch die durchgeführten Betrachtungen wurde es möglich einen „Lebensraum“ der Blasen einzugrenzen (Abbildung 4.6). Die berechneten Blasentrajektorien zeigen auf, dass auch Blasen, die zunächst außerhalb des Lebensraums liegen, beschleunigt werden und das stabile Gebiet erreichen können. Die in Zusammenarbeit mit APPEL [83] experimentell bestimmten Blasenradien (Abschnitt 5.4) bestätigen zudem die hier theoretisch abgegrenzten Ruheradien.

Das Schallfeld, das die Kavitationsblasen in ihrer radialen Oszillation und ihrer translatorischen Bewegung antreibt, ließ sich für einfache Geometrien analytisch angeben, bei aufwendigeren Geometrien wurde die Methode der Finiten Elemente zur Berechnung eingesetzt. Zudem wurde die Resonanzkurve der in der vorgelegten Arbeit vorrangig eingesetzten quaderförmigen Küvette (Abbildung 3.5) in guter Übereinstimmung zu experimentellen Messungen numerisch bestimmt.

Für den Fall nicht blasenfreier Flüssigkeiten wurde die Wellengleichung modifiziert (Abschnitt 3.3). Eindimensionale Lösungen zeigten eine abschirmende Wirkung einer zunehmenden Blasenkonzentration auf. Die Rechnungen zeigten eine

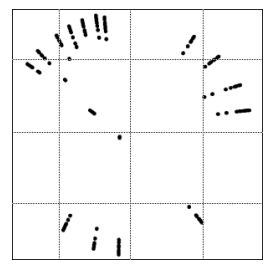


einlaufende Welle, die zum Teil an einer „Blasenwand“ schallweich reflektiert wurde und zum Teil diese Barriere durchdrang (Abbildung 3.7). Das Modell wurde linearisiert, um numerische Probleme in der nichtlinearen Beschreibung extrem schneller Dynamik (Blasenkollaps) zu vermeiden. Die Lösung des resultierenden Modells lieferte die erwartete Resonanzverschiebung (Abbildungen 3.10 und 3.11). Auch das bereits von Krefting [19] beschriebene Phänomen der Resonanzverschiebung beim Auftreten einer einzelnen Blase wurde prinzipiell nachgebildet (Abbildung 3.15). Abschließend wurde das laufende Wellenfeld unterhalb eines Kolbenstrahlers in Abschnitt 3.4 bestimmt.

Die Bjerkneskräfte wurden unter Berücksichtigung des auftretenden laufenden Wellenanteils verallgemeinert. Die Diskussion der primären Bjerkneskraft zeigte eine jetzt mögliche Beschleunigung der Blase entlang der Ausbreitungsrichtung der laufenden Welle (Abschnitt 4.2). Weiterhin wurde die Blasen-BlasenWechselwirkung unterschiedlich pulsierender Kavitationsblasen betrachtet. In bestimmten Parameterbereichen konnte eine Vorzeichenumkehr der sekundären Bjerkneskraft aufgezeigt werden (Abbildungen 4.13 und 4.14). Die detaillierte Betrachtung der sekundären Bjerkneskraft zeigte, dass auftretende Effekte durch Laufzeitverzögerungen sowie mögliche Phasendifferenzen vernachlässigt werden können.

Zum theoretischen Verständnis und zur numerischen Simulation von Blasenstrukturen wurde ein „Partikelmodell“ der Blasenbewegungen aufgestellt (Kapitel 4) und an Beispielen gelöst (Kapitel 5).

Das aufgestellte Partikelmodell lieferte gute Übereinstimmungen zwischen Experiment und Simulation für die Bewegung einer lasererzeugten Einzelblase (Abschnitt 5.1). In Abschnitt 5.2 wurde die Blasen-Blasen-Wechselwirkung zweier mit aufeinanderfolgenden Laserschüssen induzierter Kavitationsblasen sehr gut modelliert. Auch in der experimentellen Beobachtung (Abbildung 5.31) und der zugehörigen Simulation (Abbildung 5.32) wurde die Auswirkung der sekundären Bjerkneskraft deutlich. Das Ergebnis der Simulation bestätigt hier den in die Modellierung eingehenden Wechselwirkungsterm.

Die von ApPEL et al. [89] aufgenommenen dreidimensionalen Streamerfiguren wurden in Abschnitt 5.3 durch die Partikelmodellierung in einem Stehwellenfeld nachgebildet. Von KREFTING [19] experimentell präparierte akustische Lichtenbergfiguren ließen sich ebenfalls modellieren (Abschnitt 5.7). In Langzeitbeobachtungen dieser Figuren zeigten sich charakteristische ineinander überwechselnde Strukturen (Abbildung 5.41). Diese konnten mit einer Resonanzverschiebung des Resonators in Verbindung gebracht werden (Abbildung 5.40).

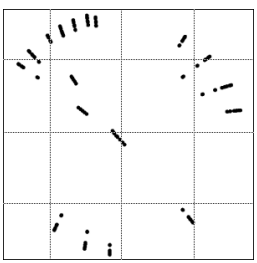


Durch das Wechselspiel von Blasendrift, Resonanzverschiebung, Schallfeldmodulation und Blasenquellenaktivität ließen sich niedrigfrequente Fluktuationen zwischen diesen Strukturen erklären und numerisch nachvollziehen.

Doppelschichtige Blasenstrukturen, die in Schallfeldern mit benachbarten Druckbäuchen auftreten, konnten in Abschnitt 5.5 mit der Simulation nachgebildet werden.

\section{Ausblick}

Mit der vorgelegten Arbeit wurde versucht, sich dem Verständnis der Strukturbildung und ihrer numerischen Modellierung zu nähern. Bis zu einer vollständigen Simulation der Phänomene sind noch offene Fragestellungen zu behandeln.

Die Einbeziehung von Strömungen in der Flüssigkeit in das hier vorgestellte Gesamtmodell wäre ein nächster wichtiger Schritt. Stärkere Flüssigkeitsströmungen können wesentlichen Einfluss auf die Blasenstrukturbildung nehmen (HATANAKA et al. [93]).

Einen erheblichen Einfluss auf die Struktur nimmt auch die Verteilung der Blasenquellen. Die bisherige Implementation geht von einer statischen Verteilung dieser Quellpunkte aus. Neben einer Modellierung der Translationsdynamik von Blasenkeimen wäre auch die Emissionsrate der aus diesen Keimen neu entstehenden Blasen genauer zu betrachten.

Die langzeitliche Entwicklung der Schallfelder und entsprechend der Blasenstrukturen kann auch deutlich von der Temperatur abhängen. Durch den längeren Betrieb eines Ultraschallbades steigt die Wassertemperatur beispielsweise an und damit die Schallgeschwindigkeit. Somit wäre die Einführung der Temperatur als variable Größe von Interesse.

Für eine Konkretisierung der Reinigungsanwendung ist die Auswirkung eingetauchter Objekte in die Flüssigkeit zu betrachten. Diese zu reinigenden Gegenstände werden ebenfalls Einfluss auf das Schallfeld sowie die mögliche Blasenanordnung nehmen. Da es sich im Allgemeinen um komplizierte Geometrien handelt, ist dafür eine Modellierung mit der Methode der Finiten Elemente notwendig.

Die bisherige Modellierung der wechselseitigen Blasen-Schallfeld-Wechselwirkung wurde durch die linearisierte Keller-Miksis-Gleichung sowie das linearisierte Wijngaarden-Modell beschrieben. Um das realistischere nichtlineare System im Dreidimensionalen durch die Methode der Finiten Elemente lösen zu können,

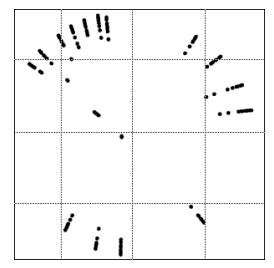


müssen noch numerische Schwierigkeiten überwunden werden. Es wäre zum Beispiel eine Möglichkeit, die Keller-Miksis-Gleichung durch eine Variablentransformation zu glätten (PARLITZ et al. [94, 30]). Auch die Verwendung eines robusteren Lösers im Zeitbereich wäre denkbar, der dem extremen Kollapsverhalten im Verlauf der Blasenpulsation folgen kann. Die Berücksichtigung der Nichtlinearität in der Wellengleichung ist ein zentraler Punkt für zukünftige Arbeiten.

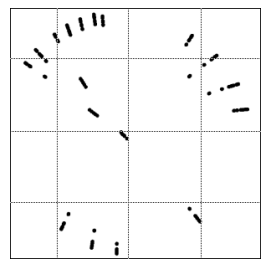




\section{Anhang A}

\section{Symbolverzeichnis}

Dieses Verzeichnis gibt die in der vorliegenden Arbeit gebräuchlichen Schreibweisen an sowie die eingesetzen Einheiten und die üblichen Wertebereiche der Größen. Die Flüssigkeitsangaben beziehen sich im Allgemeinen auf Wasser.

Die angegebenen Standardwerte grenzen den betrachteten Parameterraum ein und geben zum Teil einfache Zusammenhänge der physikalischen Größen an.

Die Sortierung der Symbole erfolgt alphabetisch.

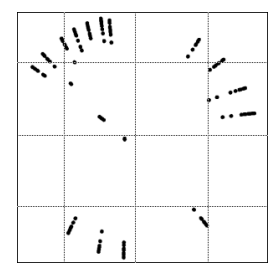




\begin{tabular}{|c|c|c|c|}
\hline Symbol & Standardwert & Einheit & Bedeutung \\
\hline$a_{l}$ & $\leq R_{\mathrm{n}}$ & $\mathrm{m}$ & Oberflächenauslenkung \\
\hline$c$ & & $\mathrm{~mol} / \mathrm{m}^{3}$ & Gaskonzentration \\
\hline$c_{\text {sat }}$ & $0.76 \ldots 1.4$ & $\mathrm{~mol} / \mathrm{m}^{3}$ & Sättigungskonzentration \\
\hline$c_{\infty}$ & $(0.1 \ldots 1) \cdot c_{\mathrm{sat}}$ & $\mathrm{mol} / \mathrm{m}^{3}$ & $\begin{array}{l}\text { Gaskonzentration in der Flüssigkeit } \\
\text { weit weg von der Blase }\end{array}$ \\
\hline$C_{1}$ & 1482 & $\mathrm{~m} / \mathrm{s}$ & Schallgeschwindigkeit im Liquid \\
\hline$C_{\mathrm{m}}$ & $<C_{1}$ & $\mathrm{~m} / \mathrm{s}$ & $\begin{array}{l}\text { Schallgeschwindigkeit im Gas- } \\
\text { Flüssigkeitsgemisch }\end{array}$ \\
\hline $\overrightarrow{d^{i} \rightarrow j}$ & $\vec{x}^{j}-\vec{x}^{i}$ & $\mathrm{~m}$ & Blasenabstand \\
\hline$D$ & $3.26 \cdot 10^{5}$ & $\mathrm{~kg} / \mathrm{m}^{3} / \mathrm{s}$ & Dämpfungskonstante \\
\hline$D_{\mathrm{R}}$ & $2 \ldots 5.1$ & $10^{9} \mathrm{~m}^{2} / \mathrm{s}$ & Diffusionsrate \\
\hline$f$ & 3 oder 5 & - & Anzahl der Molekülfreiheitsgrade \\
\hline$k$ & $2 \pi / \lambda$ & $1 / \mathrm{m}$ & Wellenzahl \\
\hline$k_{\mathrm{H}}$ & & $\mathrm{Pa} \mathrm{m}^{3} / \mathrm{mol}$ & Henry'sche Konstante \\
\hline$l$ & 2 & - & Oberflächenmode \\
\hline$p$ & & $\mathrm{~Pa}$ & Druckterm \\
\hline$p_{\mathrm{a}}$ & & $\mathrm{Pa}$ & Druck im umgebenden Fluid \\
\hline$p_{\mathrm{A}}$ & $0 \ldots \hat{p}_{\mathrm{A}}$ & $\mathrm{Pa}$ & Druckamplitude am Ort der Blase \\
\hline$\hat{p}_{\mathrm{A}}$ & $50 \ldots 200 \mathrm{k}$ & $\mathrm{Pa}$ & maximale Schalldruckamplitude \\
\hline$p_{\mathrm{i}}$ & & $\mathrm{Pa}$ & Gasdruck im Blaseninneren \\
\hline$p_{\text {stat }}$ & $100 \mathrm{k}$ & $\mathrm{Pa}$ & statischer Umgebungsdruck \\
\hline$\tilde{p}$ & $p_{\mathrm{A}} \sin (\omega t)$ & $\mathrm{Pa}$ & eingekoppelter Schallwechseldruck \\
\hline$p_{\mathrm{v}}$ & 2330 & $\mathrm{~Pa}$ & Dampfdruck \\
\hline$p_{\infty}$ & & $\mathrm{Pa}$ & $\begin{array}{l}\text { Druck im ungestörten Fluid } \\
\text { weit weg von der Blase }\end{array}$ \\
\hline$R$ & $1 \ldots 100 R_{\mathrm{n}}$ & $\mathrm{m}$ & Radius der Blase \\
\hline$R_{\mathrm{n}}$ & $1 \ldots 20 \mu$ & $\mathrm{m}$ & $\begin{array}{l}\text { Ruheradius der Blase } \\
\text { (evtl. langsam veränderlich) }\end{array}$ \\
\hline$R_{0}$ & $R_{\mathrm{n}}(t=0)$ & $\mathrm{m}$ & Initialisierungsruheradius der Blase \\
\hline$t$ & & s & Zeit \\
\hline$u$ & & $\mathrm{~m} / \mathrm{s}$ & Schnelle eines Fluidelements \\
\hline$U$ & & $\mathrm{~m} / \mathrm{s}$ & Geschwindigkeit der Blasenwand \\
\hline$V$ & $4 / 3 \pi R^{3}$ & $\mathrm{~m}^{3}$ & Volumen der Blase \\
\hline
\end{tabular}




\begin{tabular}{rrll} 
Symbol & Standardwert & Einheit & Bedeutung \\
\hline$\gamma$ & $(f+2) / f$ & - & Adiabatenexponent \\
$\eta$ & $1 \ldots \gamma$ & - & Polytropenexponent \\
$\Theta$ & 298.15 & $\mathrm{~K}$ & absolute Temperatur $\left(25^{\circ} \mathrm{C}\right)$ \\
$\lambda$ & $C_{\mathrm{l}} / \nu$ & $\mathrm{m}$ & Wellenlänge \\
$\mu$ & 0.001 & $\mathrm{Ns} / \mathrm{m}^{2}$ & dynamische Viskosität \\
$\nu$ & $20 \ldots 40$ & $\mathrm{kHz}$ & Frequenz des Schallfeldes \\
$\nu_{\mathrm{res}}^{\operatorname{lin}}$ & $\approx 3 \mathrm{~m} / \mathrm{s} / R_{\mathrm{n}}$ & $\mathrm{Hz}$ & lineare Resonanzfrequenz \\
$\varrho_{\mathrm{g}}$ & $<\varrho_{\mathrm{l}}$ & $\mathrm{kg} / \mathrm{m}^{3}$ & Dichte des Gases \\
$\varrho_{\mathrm{l}}$ & 998 & $\mathrm{~kg} / \mathrm{m}^{3}$ & Dichte der Flüssigkeit \\
$\varrho_{\mathrm{m}}$ & $\varrho_{\mathrm{g}}<\varrho_{\mathrm{m}}<\varrho_{\mathrm{l}}$ & $\mathrm{kg} / \mathrm{m}^{3}$ & Dichte des Gemisches \\
$\sigma$ & 0.07275 & $\mathrm{~N} / \mathrm{m}$ & Oberflächenspannung \\
$\tau_{\text {diff }}$ & $\gg \tau_{\mathrm{osz}}$ & $\mathrm{s}$ & Diffusionszeitskala \\
$\tau_{\mathrm{osz}}$ & & $\mathrm{s}$ & Oszillationszeitskala \\
$\tau_{\text {trans }}$ & $\gg \tau_{\mathrm{osz}}$ & $\mathrm{s}$ & Translationszeitskala \\
$\varphi$ & $-\pi \ldots \pi$ & $\mathrm{rad}$ & Phasenlage des Schallfeldes $\varphi=\varphi(\vec{x}, t)$ \\
$\omega$ & $2 \pi \nu$ & $\mathrm{rad} / \mathrm{s}$ & Kreisfrequenz \\
$\Omega$ & $2 \ldots 3$ & - & Faktor der Blake-Schwelle
\end{tabular}

\section{A.1 Tabellenwerte}

Die hier angegebenen Tabellenwerte stammen aus der Arbeit von TöGEL und LoHse [54], angegeben sind sie für eine Temperatur von $\Theta=298.15 \mathrm{~K}$ :

\begin{tabular}{lccc} 
Spezies & $f$ & $D_{\mathrm{R}}\left[10^{-9} \mathrm{~m}^{2} / \mathrm{s}\right]$ & $c_{\mathrm{sat}}\left[\mathrm{mol} / \mathrm{m}^{3}\right]$ \\
\hline Wasserstoff $\mathrm{H}_{2}$ & 5 & 5.1 & 0.78 \\
Sauerstoff $\mathrm{O}_{2}$ & 5 & 2.4 & 1.3 \\
Stickstoff $\mathrm{N}_{2}$ & 5 & 2 & 0.6 \\
Argon Ar & 3 & 2.5 & 1.4 \\
Luft & & 2.09 & 0.76
\end{tabular}

\section{A.2 Naturkonstanten}

\begin{tabular}{rrll} 
Symbol & Wert & Einheit & Benennung \\
\hline$R_{\mathrm{g}}$ & $N_{\mathrm{A}} \cdot k_{\mathrm{B}}$ & $\mathrm{J} / \mathrm{K} / \mathrm{mol}$ & universelle Gaskonstante \\
$N_{\mathrm{A}}$ & $6.022 \cdot 10^{23}$ & $1 / \mathrm{mol}$ & Avogadro-Konstante \\
$k_{\mathrm{B}}$ & $1.381 \cdot 10^{-23}$ & $\mathrm{~J} / \mathrm{K}$ & Boltzmann-Konstante \\
$g_{\mathrm{z}}$ & 9.81 & $\mathrm{~m} / \mathrm{s}^{2}$ & Gravitationsbeschleunigung
\end{tabular}

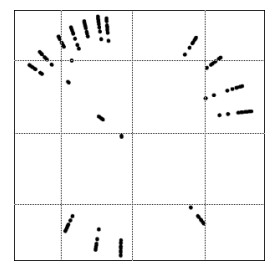




\section{A.3 Kennzahlen}

\begin{tabular}{rll} 
Symbol & Name & Referenz \\
\hline$P e$ & Péclet-Zahl & $(2.28)$
\end{tabular}

Re Reynolds-Zahl (4.8)

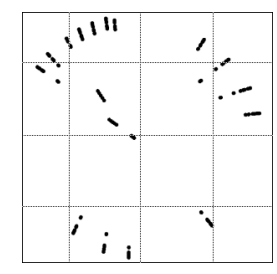




\section{Anhang B \\ Gerätespezifikationen}

Die in dieser Arbeit aufgeführten experimentellen Messungen wurden mit den hier spezifizierten Geräten durchgeführt.

\section{B.1 Küvettensysteme}

- Funktionsgenerator - Hewlett Packard 33120 A

eingesetzte Signalform: sinus

eingesetzter Frequenzbereich: $10 \ldots 40 \mathrm{kHz}$

- Hellma-Küvette mit Piezoring - Kommerziell beziehbare Küvette (Hellma 704.003) aus optischem Glas (B270 Superwite von Schott Desag):

Innenabmessung: $5 \times 5 \times 5 \mathrm{~cm}^{3}$

Wandstärke: $2.5 \mathrm{~mm}$

Als Schallwandler ist ein Piezoring (pi ceramics, PIC 151) mittig unter die Bodenplatte geklebt (Uhu endfest 300):

Innendurchmesser: $10 \mathrm{~mm}$

Außendurchmesser: $20 \mathrm{~mm}$

Höhe: $0.5 \mathrm{~mm}$

Befüllt ist die Küvette mit Wasser, das unter Umständen destilliert und entgast wurde (Gasgehalt $10 \ldots 100 \%$ ).

Das Eigenmodenspektrum dieses in Abbildung $3.2 \mathrm{zu}$ sehenden Systems wurde in Abschnitt 3.2 untersucht.

- Plexiglasquader - Abbildung B.1 zeigt den quaderförmige Eigenbau aus PMMA, der von APPEL [83] verwendet und beschrieben wurde:

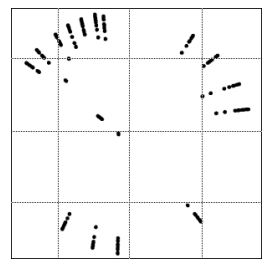


Innenabmessung: $47 \times 47 \times 61 \mathrm{~mm}^{3}$

Wandstärke: $2 \mathrm{~mm}$

zentriert unter der Bodenplatte ist ein Piezokeramik (pi ceramics, PIC 155) verklebt (Uhu endfest 300):

Durchmesser: $42 \mathrm{~mm}$

Höhe: $12 \mathrm{~mm}$

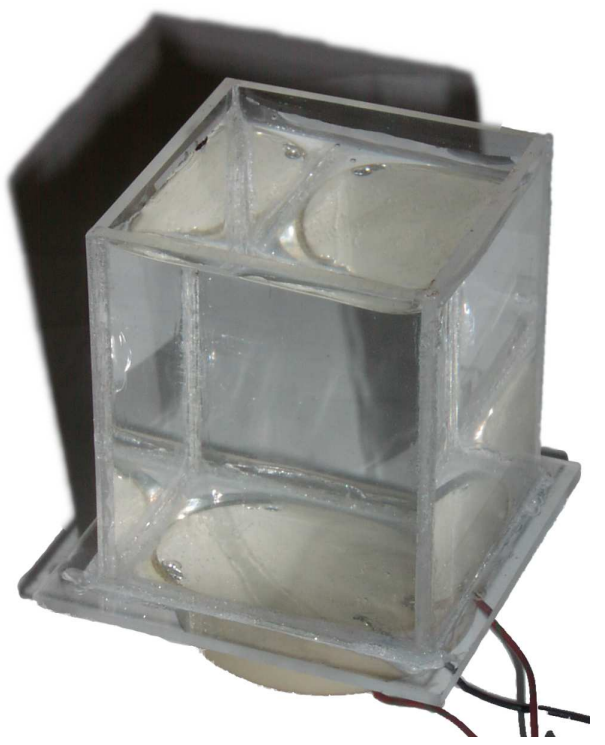

Abbildung B.1: Quaderförmige Küvette aus PMMA; Foto aus APPEL [83].

- Krohn-Hite - 50 Watt Verstärker (Krohn-Hite DCA-50)

Durch eine Impedanzanpassung wird eine maximale Leistungseinkopplung vom Verstärker in das System bestehend aus der Piezokeramik und der befüllten Küvette erreicht.

- Elac-Reaktor - Industrieller Ultraschallreaktor (ELAC nautic LVG 6102MOD), betrieben mit dem zugehörigen Verstärker.

Die Seitenwände sind im Eigenbau der mechanischen Werkstatt [95] umgerüstet auf transparenten PMMA-Aufsatz.

innere Bodenfläche: $190 \times 155 \mathrm{~mm}^{2}$

Wandhöhe: $200 \mathrm{~mm}$

Füllhöhe: $\approx 180 \mathrm{~mm}$

Wandstärke: $5 \mathrm{~mm}$

akustische Leistung: bis $700 \mathrm{~W}$

Frequenz: $\quad 40 \mathrm{kHz}$

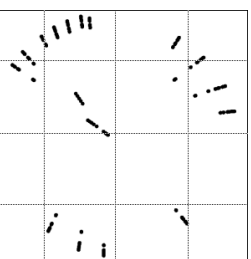




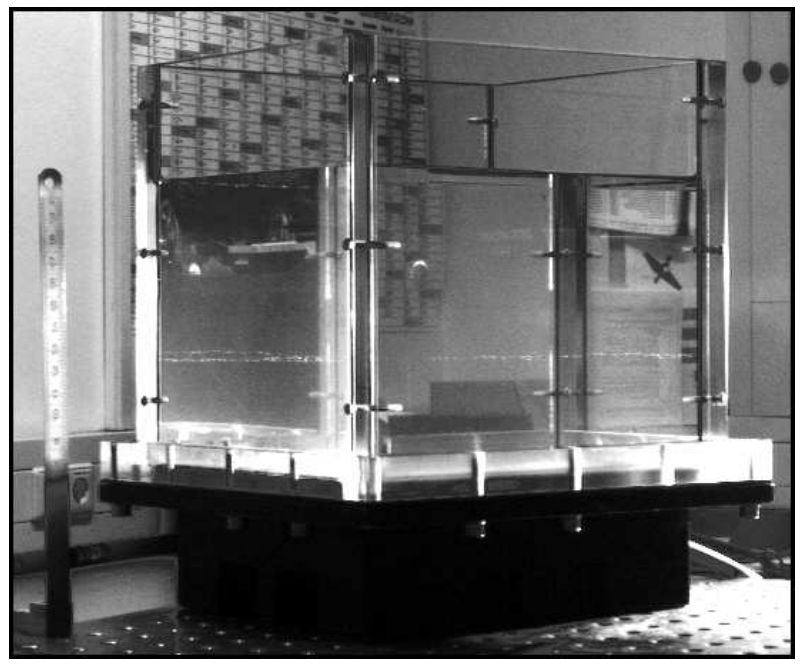

Abbildung B.2: Der Elac-Reaktor. In der Bodenplatte befinden sich 12 Ultraschallwandler; der transparente Aufsatz wurde von der mechanischen Werkstatt [95] gefertigt.

Über 12 in die Bodenplatte angeordnete Ultraschallwandler wird das Schallfeld eingekoppelt. Die Leistung kann nur Stufenweise gewählt werden, wobei in der niedrigsten Einstellung bereits die Kavitationsschwelle überschritten ist.

- Branson - Ultraschallhorn-System mit zugehörigem Verstärker vom Typ Branson Sonifier 450L. Die Konstruktion des Horns ist auf eine Bewegung der Spitze entlang der Symmetrieachse ausgelegt. Der Verstärker bietet die nicht weiter beschriebenen Leistungsstufen 1 bis 10. Das System kann kontinuierlich oder gepulst betrieben werden.

$\begin{aligned} \text { Frequenz: } & 20 \mathrm{kHz} \\ \text { Hornmaterial: } & \text { Titan } \\ \text { Durchmesser der Hornspitze: } & 12.7 \mathrm{~mm} \\ \text { Pulstakt: } & 1 \mathrm{~s} \\ \text { Pulsdauer pro Sekunde: } & 0.1,0.2 \ldots 1 \mathrm{~s}\end{aligned}$

\section{B.2 fs-Laser System}

- fs-Laser - Femtosekunden Lasersystem von Spectraphysics bestehend aus einem Tsunami Seedlaser mit Spitfire als Verstärker und Fokussierungsoptik.

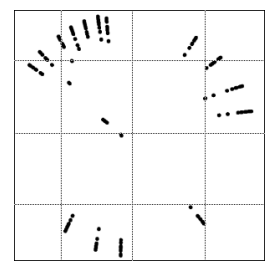




\author{
Wellenlänge: $\quad \lambda=810 \mathrm{~nm}$ \\ Energieeintrag: $<1 \mu \mathrm{J}$ pro Schuss \\ Pulsdauer: $130 \mathrm{fs}$ \\ eingesetzte Wiederholrate: > $0.889 \mathrm{~ms}$
}

\title{
B.3 Beleuchtungen
}

Durch die Beleuchtung mit Streulicht in Vorwärtsrichtung vor einem schwarzen Hintergrund werden größtmögliche Intensität und Kontrast erreicht. Die Objekte streuen dabei Licht in das Objektiv. Im Durchlicht erscheinen die Objekte als dunkle Bereiche vor der Hintergrundbeleuchtung.

- Delay - Stanford Research Systems (DG 535). Einstellbarer Generator zur Signalverzögerung.

- Trigger - Logikschaltung zur Auslösung des LED-Blitzes. Das Triggersignal des Funktionsgenerators gibt den Blitz frei, woraufhin er durch die Kamera getriggert ausgelöst werden kann. Diese Schaltung ist ein Eigenbau der elektronischen Werkstatt [96].

- LED - Dioden-Blitz; Eigenbau der elektronischen Werkstatt [96].

Blitzdauer: $2 \ldots 7 \mu \mathrm{s}$

- Halogen - handelsüblicher Halogenstrahler zur Dauerbeleuchtung.

\section{B.4 Aufnahmesysteme}

\section{B.4.1 Kameras}

- HiSIS - Hochgeschwindigkeits-CCD-Kamera (HiSIS 2002):

\section{Mode $1 \quad$ Mode 2}

Bildpunkte: $256 \times 256$ Pixel $256 \times 128$ Pixel

maximale Bildaufnahmerate: $1125 \mathrm{~Hz} \quad 2250 \mathrm{~Hz}$

minimaler Bildabstand: $888 \mu \mathrm{s}$

Belichtungszeit: $\approx 380 \mu \mathrm{s}$

Arbeitsspeicher: 512 MByte (maximal)

Aufnahmedauer: $7.28 \mathrm{~s}$ (maximal)

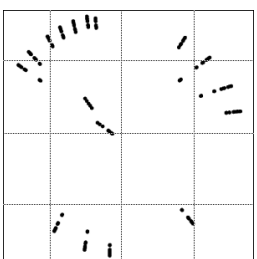


- Pulnix - CCD-Kamera (Pulnix TM-6701-AN):

Bildpunkte: $640 \times 480$ Pixel

maximale Bildaufnahmerate: $60 \mathrm{~Hz}$ minimaler Bildabstand: $16.7 \mathrm{~ms}$

- Imacon - Höchstgeschwindigkeits-CCD-Kamera (Imacon 468):

Aufösung: $576 \times 384$ Pixel

maximale Bildaufnahmerate: $100 \mathrm{MHz}$ minimaler Bildabstand: $0.01 \mu \mathrm{s}$

Aufnahmedauer: 8 Bilder (maximal)

- Phantom - hochaufösende Hochgeschwindigkeits CCD-Kamera (Phantom $\mathrm{V})$ :

maximale Auflösung: $1024 \times 1024$ Pixel

Bildaufnahmerate: $95 \mathrm{kHz}$ (maximal)

$30 \ldots 1200 \mathrm{~Hz}$ (bei höchster Auflösung)

Belichtungszeit: $\geq 10 \mu$ s (kontinuierlich einstellbar)

$5 \mu \mathrm{s}$ (minimal)

von Chow [97] eingesetzte Betriebsart:

Auflösung: $512 \times 512$ Pixel

Bildaufnahmerate: $3700 \mathrm{~Hz}$

Belichtungszeit: $61 \mu \mathrm{s}$

Arbeitsspeicher: 1 GByte

Aufnahmedauer: $1 \mathrm{~s}$

\section{B.4.2 Objektive}

Es können verschiedene Objektive mit den Kameras kombiniert werden:

- Computar - Zoomobjektiv (Computar M6Z1212) mit Nahlinse (B+W 55e NL 5) kombiniert, ermöglicht Aufnahmen im Makrobereich

Brennweite: $12.5-75 \mathrm{~mm}$

maximale Vergrößerung: 0.6

- Rodenstock - Telezentrisches Objektiv (Rodenstock TL 1-1L-215)

maximale Vergrößerung: 1

- Questar - Fernfeld Mikroskop (Questar QM 100)

maximale Vergrößerung: 10

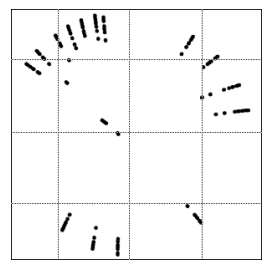




\section{B.4.3 Hydrophone}

- Faserhydrophon - optisches Glasfaserhydropon (FOPH 300)

Durchmesser der Faser: $140 \mu \mathrm{m}$

- Hydrophon - Brüel \& Kjaer (8103)

Durchmesser: $9.5 \mathrm{~mm}$

Frequenzbereich: $0.1 \mathrm{~Hz} \ldots 180 \mathrm{kHz}$

linearer Frequenzbereich: $0.1 \mathrm{~Hz} \ldots 20 \mathrm{kHz}$ mit $-1.5 \ldots 0.5 \mathrm{~dB}$

$0.1 \mathrm{~Hz} \ldots 100 \mathrm{kHz}$ mit $-3.5 \ldots 0.5 \mathrm{~dB}$

- Reson - Reson TC 4038 mit 16-bit A/D Wandlerkarte für den PC

Durchmesser: $5 \mathrm{~mm}$

Samplingfrequenz: $256 \mathrm{kHz}$

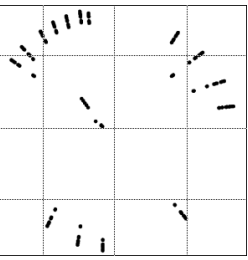




\section{Literaturverzeichnis}

[1] D. H. Trevena: Cavitation and Tension in Liquids. Adam Hilger, 1987. 1

[2] W. Lauterborn (Herausgeber): Cavitation and Inhomogeneities in Underwater Acoustics, Proceedings of the First International Conference 1979, Göttingen, Germany, 1980. Springer Verlag. 1, 2

[3] W. Lauterborn: Encyclopedia of Acoustics, Kapitel 25, Cavitation. John Wiley \& Sons, 1997. 1, 2

[4] D. Silberrad: Propeller Erosion. Engineering Seiten 33-35, 1912. 1

[5] R. T. Knapp, J. W. Daily und F. G. Hammitt: Cavitation. McGraw-Hill Book Company, 1970. 2

[6] F. R. YounG: Cavitation. McGraw-Hill Book Company, 1989. 2, 21

[7] T. G. Leighton: The Acoustic Bubble. Academic Press, 1994. 2, 83

[8] C. E. Brennen: Cavitation and Bubble Dynamics, Band 44 der Reihe $O x$ ford Engineering Science. Oxford University Press, 1995. 2

[9] A. Vogel und W. Lauterborn: Acoustic transient generation by laserproduced cavitation bubbles near solid boundaries. J. Acoust. Soc. Am. 84(2), 719-731, 1988. 2

[10] L. D. Rozenberg (Herausgeber): High-Intensity Ultrasonic Fields. Plenum Press, 1971. 2, 3

[11] T. J. Mason und J. P. Lorimer: Applied Sonochemistry, Band 1. WileyVCH, 2002. ISBN 3-527-30205-0. 2

[12] L. Bergmann: Der Ultraschall. S. Hirzel Verlag, 1954. 2

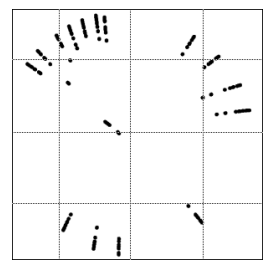


[13] T. Kodama, Y. Tomita, K.-I. Koshiyama und M. J. K. Blomley: Transfection effect of microbubbles on cells in superposed ultrasound waves and behavior of cavitation bubble. Ultrasound in Med. and Biol. 32(6), 905914, 2006. 3

[14] R. K. Schlicher, H. Radhakrishna, T. P. Tolentino, R. P. ApkaRian, V. ZARnitsyn und M. R. Prausnitz: Mechanism of intracellular delivery by acoustic cavitation. Ultrasound in Med. and Biol. 32(6), 915-924, 2006. 3

[15] M. Postema und G. Schmitz: Bubble dynamics involved in ultrasonic imaging. Exp. Rev. Molecular Diagnostics 6(3), 493-502, 2006. 3

[16] U. Parlitz, R. Mettin, S. Luther, I. Akhatov, M. Voss und W. LAUTERBorn: Spatio-temporal dynamics of acoustic cavitation bubble clouds. Phil. Trans. R. Soc. Lond. A(357), 313-334, 1999. 3, 15

[17] Y. Tomita und A. Shima: Mechanisms of impulsive pressure generation and damage pit formation by bubble collapse. J. Fluid Mech. 169, 535-564, 1986. 4

[18] A. Philipp und W. Lauterborn: Cavitation erosion by single laserproduced bubbles. J. Fluid Mech. 361, 75-116, 1998. 4

[19] D. KRefting: Untersuchung von Einzel- und Mehrblasensystemen in akustischen Resonatoren. Doktorarbeit, Drittes Physikalisches Institut der Georg-August-Universität Göttingen, 2003. 5, 28, 52, 53, 54, 55, 74, 75, $76,109,110,116,117,121,122,126,147,153,154,155,157,158,163,164$, 170,172

[20] R. Sobotta und C. Jung: Austausch im Rahmen des BMBF Projekts „Untersuchung von Kavitationsfeldern". ELMA GmbH \& Co KG, Singen. 5

[21] O. M. Lord RAyleigh: On the pressure developed in a liquid during the collapse of a spherical cavity. Phil. Mag. 34(200), 94-98, 1917. 11

[22] M. Minnaert: On Musical Air-Bubbles and the Sounds of Running Water. Phil. Mag. 16(7), 235-248, 1933. 13

[23] M. S. Plesset: The Dynamics of Cavitation Bubbles. J. Appl. Mech. 16, 277-282, 1949. 14

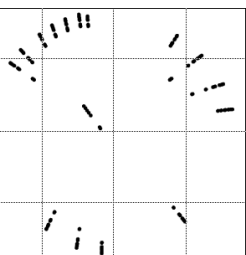


[24] H. PorItsky: The Collapse or Growth of a spherical bubble or cavity in a viscous fluid. In: 1st US National Congress on Applied Mechanics, Seiten 813-821, New York, 1952. 14

[25] J. B. Keller und I. I. Kolodner: Damping of Underwater Explosion Bubble Oscillations. J. Appl. Phys. 27, 1152-1161, 1956. 15

[26] J. B. Keller und M. Miksis: Bubble oscillations of large amplitude. J. Acoust. Soc. Am. 68(2), 628-633, 1980. 15

[27] W. Lauterborn, T. Kurz, R. Mettin und C.-D. Ohl: Advances in Chemical Physics, Band 110, Kapitel 5 Experimental and Theoretical Bubble Dynamics, Seiten 295-380. John Wiley \& Sons, 1999. 15

[28] R. Mettin, S. Luther und W. Lauterborn: Bubble size distributions and structures in acoustic cavitation. In: Proc. 2nd Conf. on Applications of Power Ultrasound in Physical and Chemical Processing, Seiten 125-129, Toulouse, France, 1999. 17

[29] A. H. Nayfeh und D. T. Mook: Nonlinear Oscillations. John Wiley \& Sons, 1979. 17

[30] U. Parlitz, V. Englisch, C. Scheffczyk und W. Lauterborn: Bifurcation structure of bubble oscillators. J. Acoust. Soc. Am. 88(2), 1061-1077, 1990. 17, 23, 174

[31] I. Akhatov, N. Gumerov, C.-D. Ohl, U. Parlitz und W. LauterBORN: The role of surface tension in stable single-bubble sonolumineszenz. Phys. Rev. Lett. 78, 227-230, 1997. 17

[32] F. G. Blake: The onset of cavitation in liquids. I. Cavitation threshold sound pressures in water as a function of temperature and hydrostatic pressure. Technischer Bericht 12, Harvard University, Acoustic Research Laboratory, 1949. 22

[33] W. Lauterborn und R. Mettin: Nonlinear Bubble Dynamics: Response Curves and More. In: L. A. CRum (Herausgeber): NATO ASI Series C: Mathematical and Physical Sciences, Band 524, Seiten 63-72, Dordrecht, 1999. Kluwer Academic Publishers. 23

[34] D. Lohse, M. P. Brenner, T. F. Dupont, S. Hilgenfeldt und B. Johnston: Sonoluminescing Air Bubbles Rectify Argon. Phys. Rev. Lett. 78(7), 1359-1362, 1997. 25

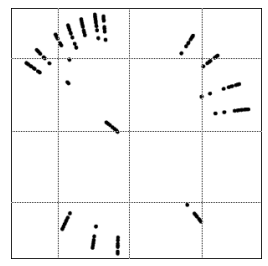


[35] D. Lohse und S. HilgenfeldT: Inert gas accumulation in sonoluminescing bubbles. J. Chem. Phys. 107(17), 6986-6997, 1997. 25

[36] C. J. Devin: Survey of Thermal, Radiation and Viscous Damping of Pulsating Air Bubbles in Water. J. Acoust. Soc. Am. 31(12), 1654-1667, 1959. 26

[37] A. I. Eller: Damping Constants of Pulsating Bubbles. J. Acoust. Soc. Am. 47(5), 1469-1470, 1970. 26

[38] A. Prosperetti: Thermal effects and damping mechanisms in the forced radial oscillations of gas bubbles in liquids. J. Acoust. Soc. Am. 61(1), 17-27, 1977. 26,27

[39] A. Prosperetti: The thermal behaviour of oscillating gas bubbles. J. Fluid Mech. 222, 587-615, 1991. 26

[40] D. Krefting, R. Mettin und W. Lauterborn: Single-Bubble Sonoluminescence in Air-Saturated Water. Phys. Rev. Lett. 91(17), 174301, 2003. 28

[41] S. Hilgenfeldt, S. Grossmann und D. Lohse: Sonoluminescence light emission. Phys. Fluids 11(6), 1318-1330, 1999. 28

[42] D. Adams: The Hitchhiker's Guide to the Galaxy. Pan Books Ltd, 1979.

[43] B. Metten: Molekulardynamik-Simulationen zur Sonolumineszenz. Doktorarbeit, Drittes Physikalisches Institut der Georg-August-Universität Göttingen, 2001. 31

[44] M. S. Plesset: On the Stability of Fluid Flows with Spherical Symmetry. J. Appl. Phys. 25(1), 96-98, 1954. 33

[45] M. S. Plesset und T. P. Mitchell: On the stability of the spherical shape of a vapor cavity in a liquid. Quart. Appl. Math. 13, 419-430, 1956. 33

[46] A. Prosperetti: Viscous effects on pertubated spherical flows. Quart. Appl. Math. 35, 339-352, 1977. 33

[47] I. N. Bronstein und K. A. Semendjajew: Taschenbuch der Mathematik. Teubner Verlagsgesellschaft, 1991. 34, 63

[48] D. Krefting, R. Mettin und W. Lauterborn: Two-frequency driven single-bubble sonoluminescence. J. Acoust. Soc. Am. 112(5), 1918-1927, 2002. 34

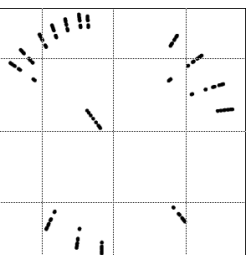


[49] A. I. Eller und H. Flynn: Rectified Diffusion during Nonlinear Pulsations of Cavitation Bubbles. J. Acoust. Soc. Am. 37(3), 493-503, 1965. 37, 40

[50] A. I. Eller: Growth of Bubbles by Rectified Diffusion. J. Acoust. Soc. Am. 46(5), 1246-1250, 1969. 41, 42

[51] L. A. CRUm: Measurements of the growth of air bubbles by rectified diffusion. J. Acoust. Soc. Am. 68(1), 203-211, 1980. 41, 42

[52] L. A. Crum: Rectified diffusion. Ultrasonics 22, 215-223, 1984. 41, 42

[53] A. I. Eller: Bubble Growth by Diffusion in a 11-kHz Sound Field. J. Acoust. Soc. Am. 52(5), 1447-1449, 1972. 42

[54] R. Tögel und D. Lohse: Phase diagrams for sonoluminescing bubbles: A comparision between experiment and theory. J. Chem. Phys. 118(4), 18631875, 2003. 42, 44, 123, 177

[55] E. Meyer und E.-G. Neumann: Physikalische und Technische Akustik. Friedr. Vieweg und Sohn Verlagsgesellschaft, 1967. 48, 50, 56

[56] P. Koch, D. Krefting, R. Mettin und W. Lauterborn: Simulation of the translational motion of few cavitation bubbles in an ultrasonic field. In: International Ultrasonics Symposium, Honolulu, USA, 2003. IEEE. 51, $129,131,137$

[57] Comsol: Comsol Multiphysics, version 3.2, 2005. Finite Elemente Simulations Software. 53, 62, 65, 69

[58] E. Meyer und D. Guicking: Schwingungslehre. Friedr. Vieweg und Sohn Verlagsgesellschaft, 1974. 54

[59] L. VAN WiJngaARDEn: On the equations of motion for mixtures of liquid and gas bubbles. J. Fluid Mech. 33(3), 465-474, 1968. 58

[60] K. W. Commander und A. Prosperetti: Linear pressure waves in bubbly liquids: Comparision between theory and experiments. J. Acoust. Soc. Am. 85(2), 732-746, 1989. 58

[61] R. E. Caflisch, M. J. Miksis, G. C. Papanicolaou und L. Ting: Effective equations for wave propagation in bubble liquids. J. Fluid Mech. 153, 259-273, 1985. 58 
[62] L. Cremer und M. Möser: Technische Akustik. Springer Verlag, 5. Auflage, 2003. 62

[63] I. Akhatov, U. Parlitz und W. Lauterborn: Towards a theory of selforganisation phenomena in bubbly-liquid mixtures. Phys. Rev. E 54(5), 49905003, 1996. 62

[64] Y. A. Kobelev und L. A. Ostrovsky: Nonlinear acoustic phenomena due to bubble drift in a gas-liquid mixture. J. Acoust. Soc. Am. 85(2), 621629, 1989. 62

[65] G. Servant, J.-L. Laborde, A. Hita, J.-P. Caltagirone und A. GÉRARD: On the interaction between ultrasound waves and bubble clouds in mono- and dual-frequency sonoreactors. Ultrasonics 10, 347-355, 2003. 62

[66] MathWorks: Matlab, version 7, 2005. Matrixorientiertes Programmpaket zur Lösung und Visualisierung mathematischer Probleme. 69

[67] G. K. Batchelor: An Introduction to Fluid Dynamics. Cambridge University Press, 2000. 83, 109

[68] D. J. Tritton: Physical Fluid Dynamics. Oxford University Press, 1988. 83,109

[69] L. D. Landau und E. M. Lifschitz: Hydrodynamik, Band 6 der Reihe Lehrbuch der Theoretischen Physik. Akademie Verlag, 5. Auflage, 1991. 83, 109

[70] V. F. K. Buerknes: Fields of Force. Columbia University Press, New York, 1906. 84

[71] I. Akhatov, R. Mettin, C.-D. Ohl, U. Parlitz und W. Lauterborn: Bjerknes force threshold for stable single bubble sonoluminescence. Phys. Rev. E 55(3), 3747-3750, 1997. 88

[72] O. Louisnard und F. Gomez: Growth by rectified diffusion of strongly acoustically forced gas bubbles in nearly saturated liquids. Phys. Rev. E 67, 036610, 2003. 89

[73] S. KAMPhAusEn: Über die sekundäre Bjerkneskraft bei zeitverzögert wechselwirkenden Kavitationsblasen. Diplomarbeit, Drittes Physikalisches Institut der Georg-August-Universität Göttingen, 1999. 98

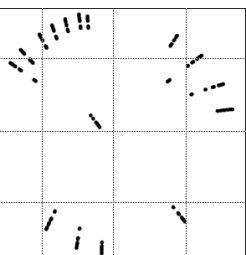


[74] R. Mettin, S. Luther, S. Kamphausen und W. Lauterborn: Dynamics of delay-coupled spherical bubbles. In: W. LAUTERBORN und T. KURZ (Herausgeber): 15th ISNA - Nonlinear Acoustics at the Turn of the Millennium, Band 524, Seiten 359-362, Göttingen, Germany, 2000. 98

[75] R. Mettin, S. Luther, C.-D. Ohl und W. Lauterborn: Simulation of bubble motion in acoustic cavitation. In: Proc. 137th ASA / 2nd EAA / Forum Acusticum Meeting, Berlin, Germany, 1999. 98

[76] C. Gerthsen, H. O. Kneser und H. Vogel: Physik. Springer Verlag, 15. Auflage, 1986. 107

[77] A. A. Doinikov: Translational motion of a spherical bubble in an acoustic standing wave of high intensity. Phys. Fluids 14(4), 1420-1425, 2002. 107

[78] L. A. Crum und A. I. Eller: Motion of Bubbles in a Stationary Sound Field. J. Acoust. Soc. Am. 48(1), 181-189, 1970. 107, 109

[79] J. Magnaudet und D. LegendRE: The viscous drag force on a spherical bubble with a time-dependent radius. Phys. Fluids 10(3), 550-554, 1998. 109

[80] A. J. REDDy und A. J. Szeri: Coupled dynamics of translation and collapse of acoustically driven microbubbles. University of California at Berkeley, Department of Mechanical Engineering, preprint. 109

[81] S. LuTHER: Theoretische Beschreibung und experimentelle Untersuchung raum-zeitlicher Strukturbildung in akustischen Kavitationsblasenfeldern. Doktorarbeit, Drittes Physikalisches Institut der Georg-August-Universität Göttingen, 2000. 112, 121, 134

[82] R. Mettin: Bubble and Particle Dynamics in Acoustic Fields: Modern Trends and Applications, Kapitel 1: Bubble structures in acoustic cavitation, Seiten 1-36. A. Doinikov, Research Signpost, India, 2005. 116

[83] J. APpel: Stereoskopische Messung von Blasentrajektorien in akustischen Stehwellenfeldern. Diplomarbeit, Drittes Physikalisches Institut der GeorgAugust-Universität Göttingen, 2002. 116, 121, 133, 134, 142, 143, 171, 179, 180

[84] R. Mettin, J. Appel, D. Krefting, R. Geisler, P. Koch und W. LAUTERBORN: Bubble structures in acoustic cavitation: observation and modelling of a "jellyfish"-streamer. In: Forum Acusticum Sevilla, Band 33. Revista de Acustica, 2002. on CDROM, ISBN 84-87985-06-8. 116, 147, 152

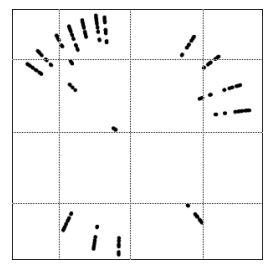


[85] R. Mettin, P. Koch, D. Krefting und W. Lauterborn: Advanced observation and modeling of an acoustic cavitation structure. In: Nonlinear Acoustics at the Beginning of the 21st Century, Band 2, Seiten 1003-1006. MSU Moscow, O. V. Rudenko and O. A. Sapozhnikov, 2002. 116

[86] R. GEISLER: Untersuchungen zur laserinduzierten Kavitation mit Nanosekunden- und Femtosekundenlasern. Doktorarbeit, Drittes Physikalisches Institut der Georg-August-Universität Göttingen, Universitätsverlag Göttingen, 2004. 118

[87] P. KocH: Simulation der Bewegung akustischer Kavitationsblasen durch ein Partikelmodell. Diplomarbeit, Drittes Physikalisches Institut der GeorgAugust-Universität Göttingen, 2001. 121

[88] D. Krefting, R. Mettin und W. Lauterborn: Translationsdynamik levitierter Einzelblasen. In: DAGA 2001, Hamburg, 2001. 122

[89] J. Appel, R. Mettin und W. Lauterborn: Stereoskopische Hochgeschwindigkeitsaufnahmen von Kavitationsblasenstrukturen. In: DAGA 2002, Bochum, 2002. 134, 135, 136, 137, 172

[90] J. Appel, P. Koch, R. Mettin, D. Krefting und W. Lauterborn: Stereoscopic high-speed recording of bubble filaments. Ultrasonics 11, 39-42, 2004. 134, 143

[91] P. Koch, R. Mettin, J. Appel, D. Krefting und W. Lauterborn: Cavitation bubble structures modeled by a particle approach. In: ESS8, Milano, Italy, 2002. 137

[92] R. Mettin, P. Koch, D. Krefting und W. Lauterborn: Advanced Observation and Modeling of an Acoustic Cavitation Structure. In: 16th ISNA - Nonlinear Acoustics at the Beginning of the 21st Century, Band 2, Seiten 1003-1006, Moskau, Russia, 2002. Rudenko, O. V. and Sapozhnikov, O. A. 147,149

[93] S. Hatanaka, K. Yasui, T. Kozuka, T. Tuziuti und H. Mitome: Influence of bubble clustering on multibubble sonoluminescence. Ultrasonics 40, 655-660, 2002. 173

[94] U. PARLiTZ: Der Begriff der Resonanz und sein Zusammenhang mit der Torsion des lokalen Flusses bei Nichtlinearen Oszillatoren. Doktorarbeit, Drittes Physikalisches Institut der Georg-August-Universität Göttingen, 1987. 174

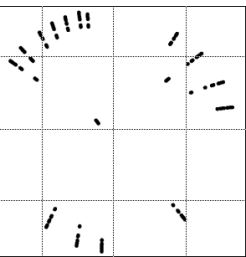


[95] Mechanische Werkstatt: Aufsatz des ELAC-Reaktors. Drittes Physikalisches Institut der Georg-August-Universität Göttingen. 180, 181

[96] Elektronische Werkstatt: Triggerschaltung und Diodenblitz. Drittes Physikalisches Institut der Georg-August-Universität Göttingen. 182

[97] R. C.-Y. Chow-McGarva: A study on the sonocrystallisation of ice. Doktorarbeit, Procter Department of Food Science, University of Leeds, UK, 2004. 183

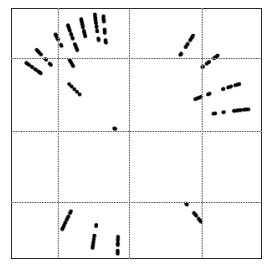





\section{Danksagung}

Bedanken möchte ich mich bei Prof. Werner Lauterborn für die Ermöglichung dieser Arbeit durch Übernahme der Betreuung und Bereitstellung der guten Infrastruktur des Instituts sowie für die Schaffung der sehr angenehmen Arbeitsatmosphäre des Hauses.

Dr. Robert Mettin danke ich für die vielen konstruktiven Anmerkungen rund um meine Arbeit sowie Prof. Ulrich Parlitz besonders für das kritische Gegenlesen.

Herrn PD Dr. Martin Rein danke ich für die freundliche Übernahme des Korreferats.

Dr. Thomas Kurz danke ich für seinen ständigen Einsatz für das angenehme und produktive Institutsleben, vom Kolloquium bis zum Kaffee. Bei Dr. Hans Werner Strube und Herrn Thomas Geiling bedanke ich mich für die sehr zuverlässig arbeitenden Soft- und Hardwareinstallationen, auf die ich mich immer verlassen konnte.

Für die schnelle Hilfe in formalen Dingen bin ich dem früheren und heutigen Sekretariat dankbar, mit Martina Schulz, Bernadette Tyson, Elke Zech und Ulrike Schwartau. Gisa Kirschmann-Schröder danke ich für die Fotoarbeiten und die netten Worte auf dem Flur.

Ich danke den stets hilfsbereiten und aufmerksamen Institutsmitgliedern und insbesondere der Nichtlinearen Dynamik Gruppe. Speziell für die enge, sehr angenehme und erfolgreiche Zusammenarbeit rings um die kleinen Blasen danke ich Dagmar Krefting, Reinhard Geisler, Jürgen Appel und Topi Tervo. Für die schöne gemeinsame Zeit im Institut danke ich Elke Hanke, Alexander Hornstein, David Engster, Jörg Dittmar, Matthias Jüschke, Karsten Köhler, Dennis Kröninger und Jann Ohle Claussen.

Für die schönen Erinnerungen an gemeinsame Forschungsaufenthalte rund um die Welt danke ich ganz herzlich Silke, Lennart und Bernhard Wolfrum, Rachel Chow und Daniel Schanz.

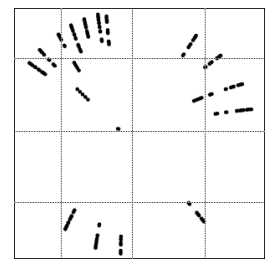


Bei Dirk Voit bedanke ich mich besonders für die vielen begeisternd lobenden und damit der Arbeit sehr zuträglichen Bemerkungen. Annette Rautenberg für die recht kurzfristige aber umso gewissenhaftere Korrektur.

Die Arbeit wurde vom Graduiertenkolleg „Strömungsinstabilitäten und Turbulenz" finanziell unterstützt.

Ein ganz besonderer Dank für die Unterstützung gebührt meinen lieben Freunden Wiebke, Claudia, Esther, Steffi, Christine, Rebekka, Kinga, Wolf und Bohle. Meiner Familie danke ich ganz herzlich für Interesse und Unterstützung für meine Arbeit und andere Lebenslagen, besonders meinen Eltern Elisabeth und Hermann, meiner Schwester Kristina und natürlich meiner Oma Tutti.

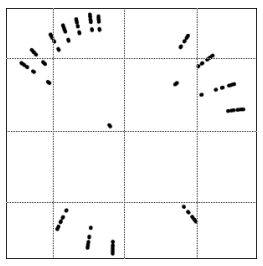




\title{
Lebenslauf
}

\author{
Name: Philipp Koch \\ Geboren: 14. Mai 1975, Göttingen \\ Nationalität: deutsch \\ 1981 - 1985 Besuch der Grundschule Dransfeld \\ 1985 - 1987 Besuch der Orientierungsstufe an der Haupt- und Realschule \\ Dransfeld \\ 1987 - 1991 Besuch der Realschule an der Haupt- und Realschule Dransfeld \\ 1991 Erwerb des Erweitertern Sekundarabschlusses 1 \\ 1991 - 1994 Besuch des Fachgymnasiums Technik an den Berufsbildenden \\ Schulen II Göttingen \\ 1994 Abschluss mit der Allgemeinen Hochschulreife \\ 1994 - 1995 Zivildienst im Universitätsklinikum Göttingen \\ 1995 - 2001 Diplomstudiengang Physik an der Georg-August-Universität \\ Göttingen \\ Juli 2001 Erwerb des Hochschulgrades Diplom-Physiker \\ Sept 2001 Beginn der Promotion Physik an der Georg-August-Universität \\ Göttingen \\ Sept 2001 - Aug 2004 Stipendiat des Graduiertenkollegs „Strömungsinstabilitäten und \\ Turbulenz" \\ Sept 2004 - Juli 2006 verschiedene Anstellungen am Dritten Physikalischen Institut der \\ Georg-August-Universität Göttingen als wissenschaftliche Hilfs- \\ kraft und Mitarbeiter
}

